\title{
OPTiMAL: A new machine learning approach for GDGT-based palaeothermometry
}

\author{
Eley, Y.1, Thomson, W. ${ }^{2}$, Greene, S.E. ${ }^{1}$, Mandel, I. ${ }^{3,4}$, Edgar, K. ${ }^{1}$, Bendle., J.A. ${ }^{1}$, and \\ Dunkley Jones, T. 1
}

\section{Supplementary Information}

This document provides more detail on the forward model described briefly in the main text. We focus on transformations for compositional data and their use, diffusion maps and the forward model. We also evaluate the performance of our nearest neighbour screening tool $\left(D_{\text {nearest }}\right)$ in light of previously available indices used to interrogate GDGT data such as the BIT, MI and RI (refs).

\section{Compositional data analysis and coordinate transformations}

Composition data are data that lie in a simplex. In other words, each datapoint must contain strictly positive entries which sum to a constant, often one. The dataset of fractional abundances of GDGTs is a compositional dataset. We refer to a single point as a composition.

The majority of multivariate statistics are designed for data that lie in Euclidean space, i.e. unconstrained space. Problems in compositional spaces may arise due to the inherent correlation between compositional parts, or models producing non-compositional predictions (e.g. uncertainty intervals extending beyond 1 for a given compositional part). For that reason, finding a mapping between the simplex and Euclidean space which allows standard multivariate techniques to be applied to compositional data has been the focus of research for decades (e.g. REFS).

There are a number of options based on log-ratio transformations. We choose the isometric log-ratio transformation for this work for the reasons laid out by Egozcue et al (2003) [1], the main reasons being that it reflects the true dimensionality of compositional data and provides a one-to-one mapping between the simplex and Euclidean space.

Given a compositional vector $x \in \mathrm{S}^{d}$ where $\mathrm{S}^{d}$ is the $d$-dimensional unit simplex, the isometric log-ratio transformation ilr : $\mathrm{S}^{d} \rightarrow \mathbb{R}^{(d-1)}$ is given by

$$
(\operatorname{ilr}(x))^{\top}=(\operatorname{clr}(x))^{\top} \Phi
$$


where $\Phi$ is a $d \times(d-1)$ matrix, $\operatorname{clr}(x)_{i}=\ln \left(\frac{x_{i}}{g(x)}\right)$ is the centred log-ratio transformation, and $g(x)$ is the geometric mean of $x$.

We use the ilr transformation as implemented by the function pivotCoord in the $\mathrm{R}$ package robCompositions. Note that ilr-transformation is unsuitable for data containing zero components. A commonly used strategy is to assume that zero values are not true zeros, but are below some detection limit imposed by the measurement technology. Thus zeros are treated as missing values and a variety of imputation methods can be employed (Martín-Fernàndez et al., 2012). We use the impCoda function in the $\mathrm{R}$ package robCompositions for this purpose.

\section{Diffusion maps for data visualisation}

Diffusion maps (Coifman et al, 2006) are a method for nonlinear dimensionality reduction and visualisation. The building block of a diffusion map is a graph representing the data, in which the vertices are datapoints. These vertices are joined by weighted edges, and a variety of choices exist for assigning weights to these edges. For the implementation we use in the main text, we represent the data as a fully connected graph (i.e., each edge has non-zero weight). The weight assigned to each edge between two points $x_{i}$ and $x_{j}$ is given by

$$
W_{i j}=\exp \left(-\frac{d^{2}\left(\boldsymbol{x}_{i}, \boldsymbol{x}_{j}\right)}{2 \sigma^{2}}\right)
$$

where $d$ is some distance function and $\sigma$ is a lengthscale parameter to be set later. Essentially this builds a graph in which points which are 'close' subject to $d$ and $\sigma$ have heavily weighted edges and points which are distant have small weights on their edges.

To properly account for the compositional nature of the data, we use a simplicial distance measure rather than the typical Euclidean distance. We choose the Aitchison distance, which is simply the Euclidean distance between ilr-transformed datapoints (Egozcue et al., 2003).

We then used these weights to define a discrete-time, discrete-distance Markov process on the data, with some transition matrix $P$ which depends on $W$ (for full details of the choice of the transition matrix we use in the main text see Haghverdi et al., 2015).

The eigenvectors of this transition matrix are referred to as diffusion components. They represent the dominant modes of variation in the data. The diffusion distance between two points reflects their connectivity in the graph and is related to the probability that one point can be reached from the other in some specified time. The advantage of representing the points by their diffusion components for visualisation purposes is that Euclidean distance in the diffusion space is approximately equivalent to diffusion 
distance in the original space (Nadler et al., 2006).

\section{Details of the forward model}

The forward model is built on multi-output (or vector-valued) Gaussian Processes. We call this model a forward model in the sense of Haslet et al. (2006), i.e., the basic building block of the model is the assumption that measured compositions arise via some unknown function of temperature plus additional temperature-independent noise. The more traditional regression strategy would be to model the outcome of interest (temperature) as a function of measured predictions (compositions), and indeed this is the approach of the Gaussian Process Regression model described in the main text.

Let $x_{i} \in \mathrm{S}^{d}$ denote the $i^{\text {th }}$ measured composition, and let $x_{i}^{*} \in \mathbb{R}^{(d-1)}$ denote the ilrtransformation of $x_{i}$. We begin with the very general model

$$
x_{i}^{*}=f\left(T_{i}\right)+\varepsilon_{i}
$$

where $f: \mathbb{R} \rightarrow \mathbb{R}^{(d-1)}$ is a function to be specified/determined and $\varepsilon_{i}$ is a zero-mean random variable independent of temperature.

The function $f$ describes the way in which sea surface temperatures give rise to steadystate GDGT compositions in populations of marine archaeota. It is clear that temperature has some effect, but a well-reasoned mechanistic model has, to our knowledge, not been developed.

In order to capture the model uncertainty associated with the lack of a mechanistic model, we take a Bayesian approach and place a multi-output Gaussian process prior on the function $f$. We also assume that $\varepsilon$ is Gaussian with diagonal covariance matrix $\Sigma$.

In other words, the forward model is as follows

$$
\begin{aligned}
x^{*} \mid T, f, \Sigma & \sim \mathcal{N}(f(T), \Sigma), \\
f & \sim \operatorname{MOGP}(\boldsymbol{K}), \\
\sigma_{i} & \propto 1, i=1, \ldots, d-1, \\
\Sigma & =\operatorname{diag}(\sigma)
\end{aligned}
$$

MOGP refers to a zero-mean, multi-output Gaussian process with kernel $\boldsymbol{K}$. There are a number of choices for kernels in multi-output Gaussian process regression models (Alvarez et al., 2012). We choose perhaps the simplest option, the Intrinsic Coregionalisation Model (ICM) with a Matern 3/2 base kernel. The model is implemented in Python 3.6 via the GPy library - the kernel hyperparameters are optimised by maximising the marginal likelihood, and probabilistic predictions are subsequently made using the exact form for the posterior. The code is available as part of the Github repository detailed in the main text. 
The most useful outcome of estimating this model is gaining access to the conditional density $p\left(x^{*} \mid T\right)$, i.e. gaining the ability to predict a distribution over compositions, given a temperature.

Armed with this distribution, a simple application of Bayes' rule allows the model to be inverted and we gain access to $p\left(T \mid x^{*}\right)$, i.e. a distribution over temperatures given a new composition. In other words, we compute

$$
p\left(T \mid x^{*}\right)=\frac{p\left(x^{*} \mid T\right) p(T)}{p\left(x^{*}\right)}
$$

Here $p(T)$ is a prior distribution over temperatures and reflects our prior beliefs about 'reasonable' sea surface temperatures. For example, we know that sea surface temperatures below $\sim-5^{\circ} \mathrm{C}$ or above $\sim 50^{\circ} \mathrm{C}$ are unreasonable, and our prior should reflect this. If a uniform prior over all temperatures is assumed (i.e. any temperature is possible a priori), the posterior is improper (i.e. does not integrate to 1) due to the large probability mass assigned to all compositions at temperatures far outside the modern temperature range.

Note that the normalising factor $p\left(x^{*}\right)=\int_{\mathbb{R}} p\left(x^{*} \mid T\right) p(T) d T$ is required to ensure that the probability distribution is properly normalised and to allow the computation of quantities of interest such as the predictive mean, $\int_{\mathbb{R}} T p\left(T \mid x^{*}\right) d T$, and variance, $\int_{\mathbb{R}}(T-\mu)^{2} p\left(T \mid x^{*}\right) d T$, of the distribution.

Since the integrals are one dimensional, it is straightforward and computationally cheap to use numerical quadrature to evaluate them.

For the applications in the paper, $p(T)$ is chosen to be a Gaussian distribution, and so the natural choice is Gauss-Hermite quadrature. We use 500 point Gauss-Hermite quadrature. For more details on quadrature in general and numerical methods for integration see Press et al. (2007).

For further details on the implementation of the model see the accompanying code. A schematic of the model is presented in Figure 1. 


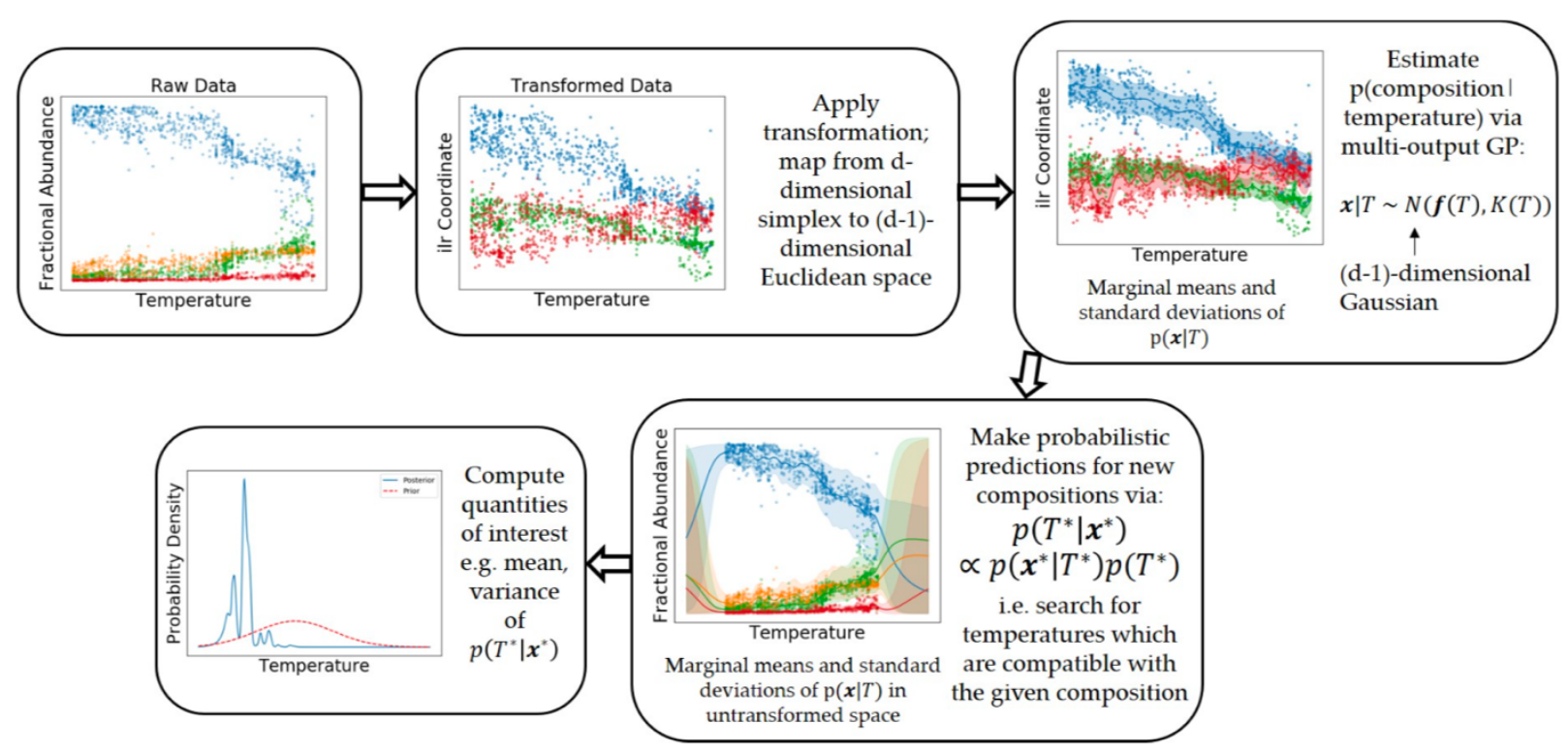

Figure 1: Schematic representation of the forward model

\section{Comparison of screening methods for GDGT assemblages}

Existing approaches make use of a range of calculated indices to evaluate GDGT assemblages and identify any potential biases that could influence SST reconstructions. Examples of this include BIT, MI and RI (refs). While most studies report these values, it is noteworthy that they are not always used as a definitive screening process and values significantly above the respective thresholds have been deemed acceptable (e.g., Super et al., 2018). Here, we use existing compilations of Eocene and Cretaceous GDGTs (Inglis et al., 2015; O'Brien et al., 2017) to demonstrate the value of our nearest neighbour (symbol) screening tool and OPTiMAL.

We first calculated $D_{\text {nearest }}$ (as described in the main text) for the entirety of the published Eocene and Cretaceous datasets, including all values that the original studies discounted due to BIT, MI and RI exceeding their respective thresholds. We identified that $67 \%$ of Eocene and $97 \%$ of Cretaceous data fail our $D_{\text {nearest }}$ quality check (Figure 2A). We then repeated this exercise using Eocene and Cretaceous data that had passed the screening tests in the original publications (Figure 2B). From these plots it is clear that existing screening methodologies do remove samples with strongly non-analogue compositions, as defined by $D_{\text {nearest }}$ values, especially at the most extreme end with $D_{\text {nearest }}>100$. However, existing screening protocols fail to exclude many of the samples which, in our methodology, fail the $D_{\text {nearest }}$ criteria $(<0.5)$. Conversely, there are a few (quantify?) samples which pass our $D_{\text {nearest }}$ criteria $(<0.5)$ but have been excluded previously on the basis of BIT, MI or RI criteria. For the moment, we suggest that a combined approach using 
BIT, MI, RI and $D_{\text {nearest }}$ provides the greatest potential for identifying non-analogue behaviour in fossil GDGT assemblages.
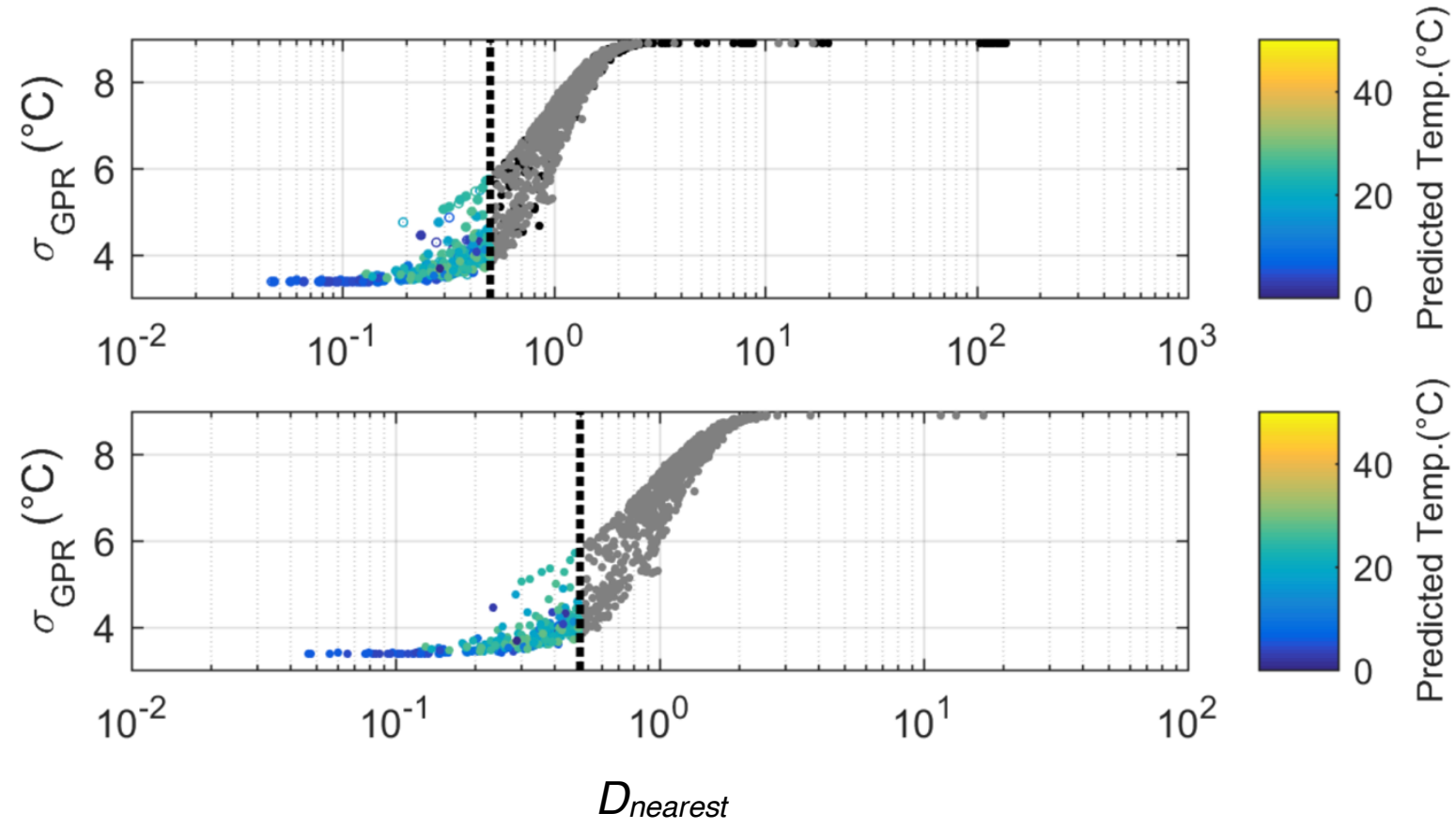

Figure 2: Top panel shows $D_{\text {nearest }}$ for all Eocene and Cretaceous data, bottom panel shows $D_{\text {nearest }}$ for those datapoints that passed screening in the original submissions (Inglis et al., 2015; O'Brien et al., 2017). Filled circles pass both $D_{\text {nearest }}$ and existing screening tests; grey circles pass existing screening tests but fail $D_{\text {nearest; }}$; black circles fail both existing screening tests and $D_{\text {nearest }}$; and open circles pass $D_{\text {nearest }}$ but fail existing screening tests.

\section{References:}

Alvarez, M.A., Rosasco, L. \& Lawrence, N.D., 2012. Kernels for vector-valued functions: A review. Foundations and Trends in Machine Learning, 4(3), 195-266.

Coifman, R.R. \& Lafon, S., 2006. Diffusion maps. Applied and computational har- monic analysis, 21(1), 5-30.

Egozcue,J.J.,Pawlowsky-Glahn,V.,Mateu-Figueras,G.\&Barcelo-Vidal,C.,2003. Isometric logratio transformations for compositional data analysis. Mathematical Geology, 35(3), 279-300. 
Haghverdi, L., Buettner, F. \& Theis, F.J., 2015. Diffusion maps for high- dimensional single-cell analysis of differentiation data. Bioinformatics, 31(18), 2989-2998.

Haslett, J., Whiley, M., Bhattacharya, S., SalterTownshend, M., Wilson, S.P., Allen, J.R.M., Huntley, B. \& Mitchell, F.J.G., 2006. Bayesian palaeoclimate reconstruction. Journal of the Royal Statistical Society: Series A (Statistics in Society), 169(3), 395438.

Inglis, G. N., Farnsworth, A., Lunt, D., Foster, G. L., Hollis, C. J., Pagani, M., Jardine, P. E., Pearson, P. N., Markwick, P., Galsworthy, A. M. J., Raynham, L., Taylor, K. W. R., and Pancost, R. D. (2015) Descent toward the Icehouse: Eocene sea surface cooling inferred from GDGT distributions, Paleoceanography, 30, 1000-1020, 10.1002/2014pa002723.

Martín-Fernàndez, J.A., Hron, K., Templ, M., Filzmoser, P. \& Palarea-Albaladejo, J., 2012. Model-based replacement of rounded zeros in compositional data: clas- sical and robust approaches. Computational Statistics \& Data Analysis, 56(9), 2688- 2704.

Nadler, B., Lafon, S., Kevrekidis, I. \& Coifman, R.R., 2006. Diffusion maps, spec- tral clustering and eigenfunctions of Fokker-Planck operators. In Advances in neu- ral information processing systems 955-962.

O'Brien, C. L., Robinson, S. A., Pancost, R. D., Sinninghe Damsté, J. S., Schouten, S., Lunt, D. J., Alsenz, H., Bornemann, 20 A., Bottini, C., Brassell, S. C., Farnsworth, A., Forster, A., Huber, B. T., Inglis, G. N., Jenkyns, H. C., Linnert, C., Littler, K., Markwick, P., McAnena, A., Mutterlose, J., Naafs, B. D. A., Püttmann, W., Sluijs, A., van Helmond, N. A. G. M., Vellekoop, J., Wagner, T., and Wrobel, N. E. (2017) Cretaceous sea-surface temperature evolution: Constraints from $\mathrm{TEX}_{86}$ and planktonic foraminiferal oxygen isotopes, Earth-Science Reviews, 172, 224-247

Press, W.H., Teukolsky, S.A., Vetterling, W.T. \& Flannery, B.P., 2007. Numerical recipes 3rd edition: The art of scientific computing. Cambridge university press. 
Modern Calibration Dataset for OPTiMAL

\begin{tabular}{|c|c|c|c|c|c|}
\hline GDGT-0 & GDGT-1 & GDGT-2 & GDGT-3 & :renarchaeo & Cren' \\
\hline 0.285 & 0.059 & 0.039 & 0.015 & 0.596 & 0.006 \\
\hline 0.283 & 0.065 & 0.043 & 0.022 & 0.587 & 0 \\
\hline 0.257 & 0.053 & 0.037 & 0.013 & 0.635 & 0.005 \\
\hline 0.3073 & 0.0561 & 0.041 & 0.014 & 0.5756 & 0.006 \\
\hline 0.3046 & 0.0581 & 0.0431 & 0.014 & 0.5731 & 0.007 \\
\hline 0.432 & 0.115 & 0.057 & 0.021 & 0.37 & 0.005 \\
\hline 0.449 & 0.029 & 0.015 & 0.005 & 0.5 & 0.002 \\
\hline 0.2667 & 0.0599 & 0.041 & 0.015 & 0.6094 & 0.008 \\
\hline 0.273 & 0.064 & 0.045 & 0.018 & 0.591 & 0.009 \\
\hline 0.2767 & 0.0629 & 0.043 & 0.016 & 0.5944 & 0.007 \\
\hline 0.283 & 0.05 & 0.034 & 0.012 & 0.613 & 0.008 \\
\hline 0.263 & 0.057 & 0.04 & 0.014 & 0.619 & 0.007 \\
\hline 0.2807 & 0.0599 & 0.049 & 0.017 & 0.5854 & 0.008 \\
\hline 0.273 & 0.059 & 0.044 & 0.015 & 0.602 & 0.007 \\
\hline 0.2687 & 0.0519 & 0.038 & 0.013 & 0.6214 & 0.007 \\
\hline 0.2677 & 0.0539 & 0.036 & 0.013 & 0.6224 & 0.007 \\
\hline 0.264 & 0.038 & 0.046 & 0.015 & 0.63 & 0.007 \\
\hline 0.2557 & 0.0529 & 0.037 & 0.014 & 0.6314 & 0.009 \\
\hline 0.27 & 0.06 & 0.041 & 0.015 & 0.606 & 0.008 \\
\hline 0.4456 & 0.0639 & 0.0639 & 0.022 & 0.3896 & 0.015 \\
\hline 0.2882 & 0.0808 & 0.0861 & 0.0141 & 0.4886 & 0.0422 \\
\hline 0.278 & 0.056 & 0.043 & 0.015 & 0.601 & 0.007 \\
\hline 0.2606 & 0.0745 & 0.0824 & 0.0133 & 0.5319 & 0.0372 \\
\hline 0.2322 & 0.0521 & 0.042 & 0.015 & 0.6517 & 0.007 \\
\hline 0.243 & 0.055 & 0.04 & 0.014 & 0.64 & 0.008 \\
\hline 0.2993 & 0.0743 & 0.075 & 0.0102 & 0.5085 & 0.0327 \\
\hline 0.3606 & 0.0599 & 0.038 & 0.014 & 0.5175 & 0.01 \\
\hline 0.3263 & 0.0581 & 0.039 & 0.013 & 0.5546 & 0.009 \\
\hline 0.1533 & 0.0499 & 0.072 & 0.0441 & 0.6527 & 0.0279 \\
\hline 0.295 & 0.052 & 0.035 & 0.011 & 0.6 & 0.007 \\
\hline 0.272 & 0.056 & 0.04 & 0.014 & 0.607 & 0.011 \\
\hline 0.2448 & 0.0569 & 0.041 & 0.015 & 0.6354 & 0.007 \\
\hline 0.2378 & 0.0559 & 0.043 & 0.016 & 0.6394 & 0.008 \\
\hline 0.269 & 0.063 & 0.047 & 0.017 & 0.594 & 0.01 \\
\hline 0.2478 & 0.0629 & 0.047 & 0.018 & 0.6114 & 0.013 \\
\hline 0.241 & 0.063 & 0.048 & 0.018 & 0.615 & 0.015 \\
\hline 0.2527 & 0.0649 & 0.049 & 0.018 & 0.6014 & 0.014 \\
\hline 0.241 & 0.062 & 0.046 & 0.017 & 0.62 & 0.014 \\
\hline 0.39 & 0.054 & 0.03 & 0.01 & 0.509 & 0.007 \\
\hline 0.387 & 0.056 & 0.029 & 0.011 & 0.512 & 0.005 \\
\hline 0.424 & 0.042 & 0.019 & 0.006 & 0.505 & 0.004 \\
\hline 0.428 & 0.035 & 0.016 & 0.005 & 0.512 & 0.004 \\
\hline 0.432 & 0.041 & 0.02 & 0.005 & 0.498 & 0.004 \\
\hline 0.3197 & 0.047 & 0.027 & 0.009 & 0.5914 & 0.006 \\
\hline 0.4625 & 0.03 & 0.012 & 0.004 & 0.4885 & 0.003 \\
\hline 0.471 & 0.031 & 0.013 & 0.004 & 0.478 & 0.003 \\
\hline 0.4635 & 0.025 & 0.009 & 0.004 & 0.4965 & 0.002 \\
\hline 0.0894 & 0.0531 & 0.0761 & 0.0534 & 0.7057 & 0.0224 \\
\hline 0.521 & 0.03 & 0.015 & 0.005 & 0.426 & 0.003 \\
\hline 0.436 & 0.027 & 0.01 & 0.004 & 0.519 & 0.004 \\
\hline 0.39 & 0.04 & 0.023 & 0.007 & 0.535 & 0.005 \\
\hline 0.391 & 0.034 & 0.016 & 0.005 & 0.551 & 0.003 \\
\hline 0.4264 & 0.033 & 0.014 & 0.004 & 0.5185 & 0.004 \\
\hline 0.4625 & 0.034 & 0.015 & 0.005 & 0.4795 & 0.004 \\
\hline 0.3023 & 0.0766 & 0.0766 & 0.0104 & 0.5031 & 0.0311 \\
\hline 0.39 & 0.031 & 0.013 & 0.004 & 0.56 & 0.002 \\
\hline 0.3666 & 0.042 & 0.023 & 0.006 & 0.5594 & 0.003 \\
\hline 0.385 & 0.069 & 0.044 & 0.015 & 0.477 & 0.01 \\
\hline 0.4016 & 0.0619 & 0.034 & 0.012 & 0.4815 & 0.009 \\
\hline 0.421 & 0.055 & 0.028 & 0.006 & 0.481 & 0.009 \\
\hline 0.3659 & 0.064 & 0.0579 & 0.0091 & 0.4787 & 0.0244 \\
\hline 0.4494 & 0.043 & 0.02 & 0.007 & 0.4745 & 0.006 \\
\hline 0.3734 & 0.038 & 0.019 & 0.006 & 0.5596 & 0.004 \\
\hline 0.3257 & 0.046 & 0.029 & 0.009 & 0.5864 & 0.004 \\
\hline 0.247 & 0.053 & 0.044 & 0.011 & 0.641 & 0.004 \\
\hline 0.2947 & 0.0801 & 0.0883 & 0.0154 & 0.4795 & 0.0421 \\
\hline 0.2995 & 0.0887 & 0.0973 & 0.0171 & 0.4548 & 0.0427 \\
\hline 0.2737 & 0.0721 & 0.0792 & 0.0113 & 0.5283 & 0.0354 \\
\hline 0.272 & 0.0772 & 0.0868 & 0.0127 & 0.5111 & 0.0402 \\
\hline 0.2705 & 0.0691 & 0.0763 & 0.0115 & 0.5381 & 0.0345 \\
\hline 0.2693 & 0.0666 & 0.0712 & 0.0155 & 0.5433 & 0.0341 \\
\hline 0.1235 & 0.0671 & 0.0859 & 0.0494 & 0.6459 & 0.0282 \\
\hline 0.277 & 0.0741 & 0.0748 & 0.0104 & 0.5281 & 0.0356 \\
\hline 0.2872 & 0.0749 & 0.0769 & 0.0103 & 0.519 & 0.0318 \\
\hline 0.17 & 0.0597 & 0.0518 & 0.0159 & 0.6826 & 0.0201 \\
\hline 0.1943 & 0.0548 & 0.0397 & 0.0136 & 0.6863 & 0.0114 \\
\hline 0.2156 & 0.0465 & 0.0297 & 0.0111 & 0.69 & 0.007 \\
\hline 0.5008 & 0.0565 & 0.0232 & 0.0054 & 0.4006 & 0.0134 \\
\hline 0.4994 & 0.06 & 0.0267 & 0.0046 & 0.3962 & 0.0132 \\
\hline 0.4539 & 0.0613 & 0.0295 & 0.0065 & 0.4284 & 0.0205 \\
\hline 0.5112 & 0.0676 & 0.0207 & 0.0032 & 0.3883 & 0.0091 \\
\hline 0.5152 & 0.0698 & 0.0209 & 0.0042 & 0.3832 & 0.0068 \\
\hline 0.4745 & 0.0658 & 0.0303 & 0.0053 & 0.4134 & 0.0106 \\
\hline 0.4833 & 0.0662 & 0.032 & 0.0052 & 0.4039 & 0.0096 \\
\hline 0.5461 & 0.0608 & 0.0197 & 0.0034 & 0.3622 & 0.0078 \\
\hline 0.5143 & 0.06 & 0.0249 & 0.0031 & 0.3896 & 0.0081 \\
\hline 0.5326 & 0.0502 & 0.0167 & 0.0032 & 0.39 & 0.0072 \\
\hline 0.1332 & 0.057 & 0.0874 & 0.0257 & 0.6258 & 0.071 \\
\hline 0.5512 & 0.0341 & 0.0123 & 0.0028 & 0.3872 & 0.0124 \\
\hline 0.5317 & 0.0322 & 0.011 & 0.0028 & 0.4109 & 0.0113 \\
\hline 0.4118 & 0.0705 & 0.0414 & 0.0051 & 0.4522 & 0.0189 \\
\hline 0.4591 & 0.0754 & 0.03 & .0064 & 40 & 0.0162 \\
\hline
\end{tabular}




\begin{tabular}{|c|c|c|c|c|c|}
\hline GDGT-0 & GDGT-1 & GDGT-2 & GDGT-3 & :renarchaeo & Cren' \\
\hline 0.4733 & 0.023 & 0.0092 & 0.0054 & 0.4846 & 0.0045 \\
\hline 0.4866 & 0.0212 & 0.0084 & 0.0047 & 0.475 & 0.0039 \\
\hline 0.4956 & 0.0206 & 0.009 & 0.0056 & 0.4655 & 0.0037 \\
\hline 0.4699 & 0.0278 & 0.0107 & 0.0075 & 0.4794 & 0.0048 \\
\hline 0.489 & 0.0213 & 0.0101 & 0.0061 & 0.4694 & 0.0042 \\
\hline 0.4749 & 0.0222 & 0.0072 & 0.0049 & 0.4865 & 0.0042 \\
\hline 0.5341 & 0.0683 & 0.0264 & 0.015 & 0.346 & 0.0102 \\
\hline 0.4968 & 0.0465 & 0.015 & 0.0106 & 0.4235 & 0.0075 \\
\hline 0.4759 & 0.023 & 0.0075 & 0.0063 & 0.4828 & 0.0046 \\
\hline 0.4965 & 0.0358 & 0.0139 & 0.0097 & 0.4392 & 0.0049 \\
\hline 0.4828 & 0.0245 & 0.0088 & 0.0071 & 0.4716 & 0.0053 \\
\hline 0.4912 & 0.0224 & 0.0065 & 0.0061 & 0.4691 & 0.0046 \\
\hline 0.4835 & 0.0209 & 0.0066 & 0.0065 & 0.4772 & 0.0053 \\
\hline 0.474 & 0.0293 & 0.012 & 0.0067 & 0.4721 & 0.0059 \\
\hline 0.4697 & 0.0199 & 0.0079 & 0.0049 & 0.494 & 0.0036 \\
\hline 0.4941 & 0.0457 & 0.0186 & 0.011 & 0.4258 & 0.0048 \\
\hline 0.4859 & 0.0464 & 0.0213 & 0.0103 & 0.4297 & 0.0064 \\
\hline 0.4871 & 0.034 & 0.0137 & 0.0082 & 0.4524 & 0.0046 \\
\hline 0.4646 & 0.0232 & 0.0079 & 0.0067 & 0.4926 & 0.0049 \\
\hline 0.4764 & 0.0375 & 0.015 & 0.0082 & 0.457 & 0.0059 \\
\hline 0.4773 & 0.0249 & 0.0099 & 0.0061 & 0.4778 & 0.004 \\
\hline 0.46 & 0.0202 & 0.0055 & 0.0055 & 0.5034 & 0.0055 \\
\hline 0.4788 & 0.0227 & 0.0076 & 0.0065 & 0.4791 & 0.0053 \\
\hline 0.4482 & 0.0434 & 0.0254 & 0.0116 & 0.4596 & 0.0118 \\
\hline 0.3918 & 0.0361 & 0.0134 & 0.0053 & 0.5428 & 0.0107 \\
\hline 0.3767 & 0.0369 & 0.0147 & 0.0061 & 0.5565 & 0.0091 \\
\hline 0.4058 & 0.0328 & 0.015 & 0.0035 & 0.5319 & 0.0109 \\
\hline 0.3819 & 0.0332 & 0.0175 & 0.0035 & 0.5527 & 0.0111 \\
\hline 0.4148 & 0.0306 & 0.0141 & 0.0038 & 0.529 & 0.0078 \\
\hline 0.365 & 0.0368 & 0.0218 & 0.0055 & 0.5582 & 0.0128 \\
\hline 0.3462 & 0.0186 & 0.0065 & 0.0035 & 0.6216 & 0.0035 \\
\hline 0.4688 & 0.0233 & 0.0078 & 0.0029 & 0.4936 & 0.0036 \\
\hline 0.4519 & 0.018 & 0.0066 & 0.0026 & 0.5173 & 0.0036 \\
\hline 0.4333 & 0.0216 & 0.0072 & 0.0022 & 0.5319 & 0.0037 \\
\hline 0.4062 & 0.0218 & 0.0077 & 0.0021 & 0.558 & 0.0042 \\
\hline 0.5419 & 0.0398 & 0.0117 & 0.0053 & 0.3975 & 0.0038 \\
\hline 0.5492 & 0.0329 & 0.0092 & 0.0042 & 0.4016 & 0.003 \\
\hline 0.5574 & 0.0261 & 0.0077 & 0.0037 & 0.4023 & 0.0028 \\
\hline 0.5517 & 0.0195 & 0.0057 & 0.0031 & 0.4178 & 0.0023 \\
\hline 0.5591 & 0.0189 & 0.006 & 0.0033 & 0.4106 & 0.0021 \\
\hline 0.4776 & 0.0135 & 0.0043 & 0.0029 & 0.4994 & 0.0022 \\
\hline 0.4971 & 0.0142 & 0.0045 & 0.0029 & 0.4794 & 0.0018 \\
\hline 0.5258 & 0.0148 & 0.0043 & 0.0026 & 0.4504 & 0.0021 \\
\hline 0.5124 & 0.0145 & 0.0044 & 0.0027 & 0.464 & 0.002 \\
\hline 0.5067 & 0.0153 & 0.0047 & 0.0028 & 0.4683 & 0.0023 \\
\hline 0.55 & 0.0172 & 0.0052 & 0.003 & 0.4225 & 0.002 \\
\hline 0.5596 & 0.0176 & 0.0053 & 0.0031 & 0.4126 & 0.0017 \\
\hline 0.5515 & 0.0198 & 0.0058 & 0.0031 & 0.4178 & 0.0021 \\
\hline 0.5642 & 0.0242 & 0.0068 & 0.0034 & 0.3991 & 0.0022 \\
\hline 0.5486 & 0.0281 & 0.0091 & 0.0042 & 0.4072 & 0.0028 \\
\hline 0.5421 & 0.0341 & 0.0104 & 0.0047 & 0.4057 & 0.0029 \\
\hline 0.5258 & 0.0389 & 0.0118 & 0.0053 & 0.4145 & 0.0037 \\
\hline 0.5436 & 0.0282 & 0.0085 & 0.0042 & 0.4126 & 0.0031 \\
\hline 0.5681 & 0.0274 & 0.0084 & 0.0037 & 0.3898 & 0.0026 \\
\hline 0.5527 & 0.021 & 0.0062 & 0.0032 & 0.4143 & 0.0025 \\
\hline 0.5431 & 0.019 & 0.0057 & 0.0032 & 0.4267 & 0.0024 \\
\hline 0.4712 & 0.0125 & 0.0042 & 0.003 & 0.5069 & 0.0022 \\
\hline 0.4798 & 0.0127 & 0.0042 & 0.003 & 0.4977 & 0.0025 \\
\hline 0.5127 & 0.0148 & 0.0048 & 0.0029 & 0.4624 & 0.0025 \\
\hline 0.5107 & 0.0158 & 0.0047 & 0.003 & 0.4639 & 0.0019 \\
\hline 0.5306 & 0.0154 & 0.0046 & 0.0027 & 0.4445 & 0.0021 \\
\hline 0.5295 & 0.0234 & 0.007 & 0.0035 & 0.4339 & 0.0028 \\
\hline 0.5183 & 0.0256 & 0.0078 & 0.0037 & 0.4415 & 0.003 \\
\hline 0.4429 & 0.0215 & 0.0082 & 0.0025 & 0.5211 & 0.0038 \\
\hline 0.4957 & 0.0233 & 0.0073 & 0.0028 & 0.4676 & 0.0034 \\
\hline 0.4313 & 0.0234 & 0.0085 & 0.0028 & 0.5308 & 0.0032 \\
\hline 0.1624 & 0.0651 & 0.0937 & 0.0237 & 0.6034 & 0.0516 \\
\hline 0.434 & 0.0846 & 0.0547 & 0.0035 & 0.4062 & 0.0169 \\
\hline 0.4052 & 0.0704 & 0.0466 & 0.0037 & 0.4606 & 0.0135 \\
\hline 0.0894 & 0.0411 & 0.1019 & 0.0264 & 0.6366 & 0.1047 \\
\hline 0.1397 & 0.0478 & 0.0931 & 0.0256 & 0.6034 & 0.0904 \\
\hline 0.4471 & 0.0779 & 0.0337 & 0.0109 & 0.4201 & 0.0103 \\
\hline 0.5434 & 0.061 & 0.0192 & 0.0039 & 0.3655 & 0.0069 \\
\hline 0.4461 & 0.0578 & 0.0334 & 0.0096 & 0.4461 & 0.007 \\
\hline 0.4682 & 0.034 & 0.0127 & 0.0032 & 0.4773 & 0.0047 \\
\hline 0.4141 & 0.0584 & 0.0268 & 0.0096 & 0.4683 & 0.0228 \\
\hline 0.3988 & 0.0759 & 0.0503 & 0.0078 & 0.4456 & 0.0217 \\
\hline 0.3716 & 0.0713 & 0.0544 & 0.0088 & 0.4631 & 0.0307 \\
\hline 0.399 & 0.0399 & 0.0267 & 0.005 & 0.516 & 0.0133 \\
\hline 0.3508 & 0.0783 & 0.0623 & 0.0086 & 0.4705 & 0.0295 \\
\hline 0.412 & 0.0783 & 0.0462 & 0.0044 & 0.4412 & 0.018 \\
\hline 0.3792 & 0.0549 & 0.0283 & 0.0069 & 0.5121 & 0.0186 \\
\hline 0.4634 & 0.0432 & 0.0123 & 0.005 & 0.4733 & 0.0029 \\
\hline 0.4901 & 0.0251 & 0.0099 & 0.0035 & 0.467 & 0.0043 \\
\hline 0.4948 & 0.0346 & 0.0172 & 0.004 & 0.4445 & 0.0049 \\
\hline 0.4901 & 0.0251 & 0.0099 & 0.0035 & 0.467 & 0.0043 \\
\hline 0.528 & 0.0321 & 0.0098 & 0.0035 & 0.4216 & 0.0049 \\
\hline 0.4632 & 0.0393 & 0.0171 & 0.0058 & 0.471 & 0.0034 \\
\hline 0.242 & 0.0689 & 0.0672 & 0.0181 & 0.5573 & 0.0464 \\
\hline 0.2343 & 0.0718 & 0.072 & 0.0195 & 0.5564 & 0.0459 \\
\hline 0.2236 & 0.0732 & 0.0777 & 0.0195 & 0.5465 & 0.0595 \\
\hline 0.2023 & 0.0696 & .0813 & .0179 & 0.5764 & 0.0524 \\
\hline
\end{tabular}


Modern Calibration Dataset for OPTiMAL

\begin{tabular}{|c|c|c|c|c|c|}
\hline GDGT-0 & GDGT-1 & GDGT-2 & GDGT-3 & :renarchaeo & Cren' \\
\hline 0.2366 & 0.0663 & 0.0781 & 0.0148 & 0.5488 & 0.0553 \\
\hline 0.2172 & 0.0643 & 0.0942 & 0.0177 & 0.5573 & 0.0494 \\
\hline 0.2074 & 0.0763 & 0.1012 & 0.0184 & 0.5411 & 0.0556 \\
\hline 0.2145 & 0.0729 & 0.0941 & 0.0179 & 0.5477 & 0.053 \\
\hline 0.2311 & 0.0734 & 0.0933 & 0.017 & 0.5364 & 0.0488 \\
\hline 0.2877 & 0.0702 & 0.0831 & 0.014 & 0.503 & 0.0419 \\
\hline 0.2899 & 0.0724 & 0.0829 & 0.0143 & 0.4977 & 0.0427 \\
\hline 0.2477 & 0.071 & 0.0803 & 0.0187 & 0.5368 & 0.0455 \\
\hline 0.285 & 0.074 & 0.08 & 0.015 & 0.515 & 0.031 \\
\hline 0.356 & 0.054 & 0.04 & 0.01 & 0.509 & 0.03 \\
\hline 0.356 & 0.062 & 0.038 & 0.013 & 0.521 & 0.01 \\
\hline 0.354 & 0.062 & 0.039 & 0.011 & 0.525 & 0.009 \\
\hline 0.295 & 0.064 & 0.064 & 0.011 & 0.54 & 0.026 \\
\hline 0.306 & 0.07 & 0.061 & 0.011 & 0.533 & 0.02 \\
\hline 0.363 & 0.059 & 0.036 & 0.01 & 0.516 & 0.016 \\
\hline 0.298 & 0.067 & 0.065 & 0.011 & 0.527 & 0.031 \\
\hline 0.293 & 0.073 & 0.067 & 0.014 & 0.52 & 0.035 \\
\hline 0.328 & 0.063 & 0.054 & 0.012 & 0.519 & 0.024 \\
\hline 0.37 & 0.059 & 0.039 & 0.014 & 0.507 & 0.012 \\
\hline 0.34 & 0.066 & 0.044 & 0.012 & 0.521 & 0.017 \\
\hline 0.274 & 0.078 & 0.066 & 0.014 & 0.537 & 0.03 \\
\hline 0.275 & 0.071 & 0.076 & 0.011 & 0.523 & 0.044 \\
\hline 0.359 & 0.063 & 0.036 & 0.013 & 0.524 & 0.005 \\
\hline 0.347 & 0.066 & 0.039 & 0.013 & 0.525 & 0.01 \\
\hline 0.302 & 0.068 & 0.055 & 0.013 & 0.528 & 0.034 \\
\hline 0.253 & 0.095 & 0.087 & 0.012 & 0.503 & 0.051 \\
\hline 0.34 & 0.069 & 0.046 & 0.013 & 0.516 & 0.015 \\
\hline 0.371 & 0.069 & 0.037 & 0.012 & 0.503 & 0.008 \\
\hline 0.375 & 0.058 & 0.029 & 0.011 & 0.51 & 0.017 \\
\hline 0.387 & 0.052 & 0.023 & 0.01 & 0.51 & 0.018 \\
\hline 0.403 & 0.048 & 0.021 & 0.009 & 0.507 & 0.011 \\
\hline 0.382 & 0.051 & 0.024 & 0.008 & 0.526 & 0.009 \\
\hline 0.373 & 0.067 & 0.029 & 0.01 & 0.511 & 0.01 \\
\hline 0.351 & 0.072 & 0.048 & 0.013 & 0.496 & 0.02 \\
\hline 0.305 & 0.071 & 0.057 & 0.014 & 0.525 & 0.029 \\
\hline 0.326 & 0.073 & 0.048 & 0.013 & 0.527 & 0.012 \\
\hline 0.377 & 0.055 & 0.027 & 0.009 & 0.523 & 0.01 \\
\hline 0.376 & 0.046 & 0.019 & 0.007 & 0.549 & 0.003 \\
\hline 0.379 & 0.055 & 0.029 & 0.01 & 0.522 & 0.006 \\
\hline 0.382 & 0.044 & 0.021 & 0.008 & 0.54 & 0.005 \\
\hline 0.305 & 0.07 & 0.059 & 0.012 & 0.528 & 0.025 \\
\hline 0.356 & 0.062 & 0.035 & 0.011 & 0.524 & 0.013 \\
\hline 0.37 & 0.06 & 0.034 & 0.01 & 0.515 & 0.01 \\
\hline 0.357 & 0.061 & 0.035 & 0.013 & 0.511 & 0.023 \\
\hline 0.324 & 0.07 & 0.051 & 0.011 & 0.521 & 0.023 \\
\hline 0.345 & 0.062 & 0.037 & 0.012 & 0.531 & 0.011 \\
\hline 0.352 & 0.063 & 0.039 & 0.011 & 0.521 & 0.013 \\
\hline 0.3 & 0.07 & 0.059 & 0.012 & 0.534 & 0.024 \\
\hline 0.346 & 0.061 & 0.04 & 0.014 & 0.539 & 0 \\
\hline 0.341 & 0.063 & 0.038 & 0.012 & 0.535 & 0.011 \\
\hline 0.338 & 0.062 & 0.037 & 0.011 & 0.539 & 0.014 \\
\hline 0.323 & 0.064 & 0.041 & 0.013 & 0.54 & 0.018 \\
\hline 0.328 & 0.065 & 0.05 & 0.012 & 0.53 & 0.016 \\
\hline 0.308 & 0.07 & 0.049 & 0.012 & 0.533 & 0.027 \\
\hline 0.3144 & 0.0844 & 0.0815 & 0.0081 & 0.4764 & 0.0351 \\
\hline 0.3162 & 0.0822 & 0.0802 & 0.0082 & 0.4808 & 0.0323 \\
\hline 0.3121 & 0.0806 & 0.0769 & 0.0082 & 0.4872 & 0.035 \\
\hline 0.3076 & 0.0823 & 0.0772 & 0.0078 & 0.4888 & 0.0364 \\
\hline 0.2972 & 0.0815 & 0.0797 & 0.0101 & 0.4949 & 0.0367 \\
\hline 0.365 & 0.0852 & 0.0687 & 0.0081 & 0.4466 & 0.0263 \\
\hline 0.2721 & 0.0665 & 0.0771 & 0.0113 & 0.5377 & 0.0353 \\
\hline 0.2617 & 0.0694 & 0.0823 & 0.0114 & 0.5358 & 0.0394 \\
\hline 0.2664 & 0.0732 & 0.0655 & 0.0155 & 0.5539 & 0.0254 \\
\hline 0.2959 & 0.0673 & 0.0659 & 0.0116 & 0.5305 & 0.0287 \\
\hline 0.2723 & 0.0701 & 0.0783 & 0.0116 & 0.5336 & 0.034 \\
\hline 0.291 & 0.0743 & 0.0832 & 0.0112 & 0.5028 & 0.0375 \\
\hline 0.2828 & 0.0708 & 0.0856 & 0.0099 & 0.5122 & 0.0387 \\
\hline 0.2889 & 0.0728 & 0.0821 & 0.0091 & 0.5099 & 0.0373 \\
\hline 0.2884 & 0.0715 & 0.0768 & 0.0085 & 0.5167 & 0.038 \\
\hline 0.2759 & 0.0677 & 0.0842 & 0.0104 & 0.5269 & 0.0349 \\
\hline 0.275 & 0.0652 & 0.0749 & 0.0116 & 0.5395 & 0.0339 \\
\hline 0.2909 & 0.0713 & 0.0872 & 0.0114 & 0.5001 & 0.0391 \\
\hline 0.2993 & 0.0748 & 0.0931 & 0.0179 & 0.4775 & 0.0375 \\
\hline 0.2506 & 0.0651 & 0.086 & 0.0175 & 0.5453 & 0.0355 \\
\hline 0.2394 & 0.0902 & 0.1557 & 0.017 & 0.4646 & 0.0331 \\
\hline 0.2596 & 0.073 & 0.0907 & 0.0098 & 0.5291 & 0.0378 \\
\hline 0.2633 & 0.0712 & 0.0948 & 0.0125 & 0.5182 & 0.0399 \\
\hline 0.2739 & 0.0812 & 0.0915 & 0.0084 & 0.5085 & 0.0365 \\
\hline 0.3274 & 0.082 & 0.0749 & 0.0062 & 0.4784 & 0.0311 \\
\hline 0.3427 & 0.0851 & 0.0742 & 0.006 & 0.4574 & 0.0346 \\
\hline 0.3134 & 0.0815 & 0.0733 & 0.0072 & 0.4908 & 0.0339 \\
\hline 0.3102 & 0.0802 & 0.0721 & 0.008 & 0.4944 & 0.035 \\
\hline 0.2957 & 0.0806 & 0.0734 & 0.0076 & 0.5073 & 0.0353 \\
\hline 0.4195 & 0.0191 & 0.0068 & 0.0027 & 0.5476 & 0.0043 \\
\hline 0.4874 & 0.0398 & 0.0176 & 0.0039 & 0.4446 & 0.0068 \\
\hline 0.4707 & 0.0557 & 0.0293 & 0.0046 & 0.4292 & 0.0106 \\
\hline 0.4808 & 0.0622 & 0.0283 & 0.0039 & 0.4142 & 0.0107 \\
\hline 0.499 & 0.0543 & 0.022 & 0.0032 & 0.414 & 0.0075 \\
\hline 0.4296 & 0.0198 & 0.0067 & 0.0026 & 0.5367 & 0.0045 \\
\hline 0.5234 & 0.041 & 0.0151 & 0.0031 & 0.4127 & 0.0047 \\
\hline 0.4995 & 0.0566 & 0.0223 & 0.0032 & 0.4111 & 0.0073 \\
\hline 0.5117 & 0.0607 & .0291 & .0035 & 0.3875 & 0.0074 \\
\hline
\end{tabular}




\begin{tabular}{|c|c|c|c|c|c|}
\hline GDGT-0 & GDGT-1 & GDGT-2 & GDGT-3 & :renarchaeo & Cren' \\
\hline 0.4928 & 0.0606 & 0.0248 & 0.0032 & 0.4093 & 0.0094 \\
\hline 0.4872 & 0.0603 & 0.0261 & 0.0035 & 0.4151 & 0.0077 \\
\hline 0.5404 & 0.0342 & 0.011 & 0.003 & 0.4074 & 0.004 \\
\hline 0.5004 & 0.0613 & 0.0291 & 0.0035 & 0.3992 & 0.0065 \\
\hline 0.5261 & 0.0325 & 0.0108 & 0.0031 & 0.4232 & 0.0044 \\
\hline 0.4948 & 0.0636 & 0.0262 & 0.0032 & 0.4035 & 0.0087 \\
\hline 0.4921 & 0.0592 & 0.0243 & 0.0035 & 0.413 & 0.008 \\
\hline 0.4738 & 0.0557 & 0.0254 & 0.0035 & 0.4314 & 0.0102 \\
\hline 0.4615 & 0.0566 & 0.0267 & 0.004 & 0.4393 & 0.012 \\
\hline 0.4995 & 0.0472 & 0.0191 & 0.0032 & 0.4247 & 0.0063 \\
\hline 0.4961 & 0.0324 & 0.014 & 0.0034 & 0.4481 & 0.006 \\
\hline 0.4737 & 0.0377 & 0.0183 & 0.0039 & 0.4586 & 0.0078 \\
\hline 0.4645 & 0.0421 & 0.0229 & 0.0042 & 0.4577 & 0.0087 \\
\hline 0.4531 & 0.0447 & 0.0249 & 0.0043 & 0.4629 & 0.0101 \\
\hline 0.4456 & 0.0491 & 0.0308 & 0.0052 & 0.4567 & 0.0126 \\
\hline 0.4045 & 0.0532 & 0.0395 & 0.0061 & 0.4803 & 0.0163 \\
\hline 0.3929 & 0.0493 & 0.0382 & 0.0065 & 0.4964 & 0.0166 \\
\hline 0.4131 & 0.0395 & 0.0261 & 0.0052 & 0.5033 & 0.0128 \\
\hline 0.4058 & 0.0261 & 0.0137 & 0.0038 & 0.5438 & 0.0069 \\
\hline 0.4318 & 0.0238 & 0.011 & 0.0037 & 0.5242 & 0.0056 \\
\hline 0.3201 & 0.0763 & 0.0744 & 0.0077 & 0.4886 & 0.0329 \\
\hline 0.314 & 0.077 & 0.078 & 0.008 & 0.4916 & 0.0314 \\
\hline 0.3167 & 0.0783 & 0.0765 & 0.0089 & 0.4847 & 0.0349 \\
\hline 0.3145 & 0.0813 & 0.0815 & 0.0078 & 0.4836 & 0.0312 \\
\hline 0.3173 & 0.0785 & 0.0759 & 0.0079 & 0.4876 & 0.0328 \\
\hline 0.3785 & 0.0674 & 0.0502 & 0.0068 & 0.4759 & 0.0212 \\
\hline 0.3717 & 0.0665 & 0.0514 & 0.0071 & 0.4784 & 0.0249 \\
\hline 0.3309 & 0.0693 & 0.0657 & 0.0081 & 0.4953 & 0.0307 \\
\hline 0.3176 & 0.0737 & 0.0723 & 0.0078 & 0.494 & 0.0346 \\
\hline 0.3088 & 0.075 & 0.0745 & 0.0079 & 0.5013 & 0.0325 \\
\hline 0.3037 & 0.0736 & 0.0781 & 0.0087 & 0.5005 & 0.0354 \\
\hline 0.2946 & 0.0728 & 0.0808 & 0.0081 & 0.5072 & 0.0364 \\
\hline 0.4372 & 0.0705 & 0.0388 & 0.0065 & 0.4328 & 0.0142 \\
\hline 0.4527 & 0.0684 & 0.0332 & 0.0047 & 0.4286 & 0.0123 \\
\hline 0.4499 & 0.0674 & 0.0338 & 0.0051 & 0.429 & 0.0148 \\
\hline 0.4576 & 0.0699 & 0.0346 & 0.005 & 0.4212 & 0.0117 \\
\hline 0.471 & 0.0684 & 0.0312 & 0.0043 & 0.4148 & 0.0103 \\
\hline 0.4704 & 0.0667 & 0.0294 & 0.0042 & 0.4186 & 0.0106 \\
\hline 0.5015 & 0.0642 & 0.0262 & 0.0034 & 0.3966 & 0.0081 \\
\hline 0.4709 & 0.0599 & 0.0316 & 0.0054 & 0.4166 & 0.0156 \\
\hline 0.4789 & 0.0648 & 0.0274 & 0.0034 & 0.4164 & 0.0091 \\
\hline 0.4968 & 0.057 & 0.0255 & 0.004 & 0.408 & 0.0087 \\
\hline 0.4786 & 0.0627 & 0.0271 & 0.0038 & 0.4189 & 0.009 \\
\hline 0.4871 & 0.0637 & 0.0288 & 0.0037 & 0.4077 & 0.0092 \\
\hline 0.4519 & 0.0693 & 0.0381 & 0.0059 & 0.4209 & 0.0138 \\
\hline 0.4224 & 0.0719 & 0.0401 & 0.0063 & 0.4443 & 0.015 \\
\hline 0.4194 & 0.0723 & 0.0417 & 0.0068 & 0.443 & 0.0169 \\
\hline 0.4392 & 0.0685 & 0.0436 & 0.009 & 0.4231 & 0.0166 \\
\hline 0.3929 & 0.0761 & 0.0488 & 0.0072 & 0.4567 & 0.0182 \\
\hline 0.3914 & 0.0745 & 0.0493 & 0.0083 & 0.4551 & 0.0214 \\
\hline 0.3763 & 0.0795 & 0.0581 & 0.0088 & 0.4554 & 0.0218 \\
\hline 0.3667 & 0.0766 & 0.057 & 0.009 & 0.4665 & 0.0241 \\
\hline 0.3562 & 0.077 & 0.0589 & 0.009 & 0.4729 & 0.0259 \\
\hline 0.3454 & 0.0798 & 0.0652 & 0.0086 & 0.4724 & 0.0286 \\
\hline 0.3397 & 0.0777 & 0.0655 & 0.0074 & 0.4826 & 0.0271 \\
\hline 0.3413 & 0.0762 & 0.0674 & 0.0072 & 0.4804 & 0.0274 \\
\hline 0.3272 & 0.0776 & 0.0693 & 0.0075 & 0.4883 & 0.0301 \\
\hline 0.2985 & 0.0751 & 0.0714 & 0.0113 & 0.5087 & 0.0351 \\
\hline 0.5178 & 0.0769 & 0.0496 & 0.0077 & 0.3303 & 0.0177 \\
\hline 0.4635 & 0.0758 & 0.0502 & 0.0072 & 0.386 & 0.0173 \\
\hline 0.4954 & 0.0649 & 0.0382 & 0.0068 & 0.3808 & 0.0138 \\
\hline 0.4806 & 0.0709 & 0.045 & 0.0071 & 0.381 & 0.0154 \\
\hline 0.4551 & 0.0748 & 0.0494 & 0.0076 & 0.3951 & 0.0181 \\
\hline 0.4523 & 0.078 & 0.053 & 0.0078 & 0.3906 & 0.0184 \\
\hline 0.5038 & 0.0694 & 0.0417 & 0.007 & 0.3616 & 0.0165 \\
\hline 0.492 & 0.0705 & 0.0454 & 0.0073 & 0.3674 & 0.0174 \\
\hline 0.489 & 0.0726 & 0.0439 & 0.0073 & 0.3714 & 0.0157 \\
\hline 0.496 & 0.0763 & 0.0457 & 0.0075 & 0.3585 & 0.016 \\
\hline 0.463 & 0.0748 & 0.0507 & 0.0074 & 0.386 & 0.0181 \\
\hline 0.4617 & 0.0768 & 0.0512 & 0.0074 & 0.3849 & 0.018 \\
\hline 0.4508 & 0.0787 & 0.054 & 0.008 & 0.3891 & 0.0193 \\
\hline 0.5819 & 0.0569 & 0.0244 & 0.0064 & 0.3203 & 0.0101 \\
\hline 0.5377 & 0.0359 & 0.015 & 0.0043 & 0.4024 & 0.0047 \\
\hline 0.5166 & 0.0393 & 0.0166 & 0.0045 & 0.4168 & 0.0062 \\
\hline 0.5209 & 0.0397 & 0.0165 & 0.0045 & 0.4129 & 0.0055 \\
\hline 0.5214 & 0.0378 & 0.0162 & 0.0046 & 0.4146 & 0.0055 \\
\hline 0.5263 & 0.04 & 0.0168 & 0.0048 & 0.406 & 0.0061 \\
\hline 0.5753 & 0.044 & 0.0186 & 0.0044 & 0.3512 & 0.0066 \\
\hline 0.5359 & 0.0411 & 0.0185 & 0.0049 & 0.3932 & 0.0063 \\
\hline 0.5259 & 0.0407 & 0.0177 & 0.0048 & 0.405 & 0.0059 \\
\hline 0.525 & 0.0403 & 0.0181 & 0.0049 & 0.4058 & 0.006 \\
\hline 0.5886 & 0.041 & 0.0156 & 0.005 & 0.3431 & 0.0066 \\
\hline 0.5453 & 0.0395 & 0.0181 & 0.0052 & 0.3862 & 0.0058 \\
\hline 0.5575 & 0.0417 & 0.0182 & 0.0051 & 0.3713 & 0.0062 \\
\hline 0.4428 & 0.0903 & 0.06 & 0.0067 & 0.381 & 0.0192 \\
\hline 0.4499 & 0.0844 & 0.0572 & 0.0069 & 0.3833 & 0.0183 \\
\hline 0.4964 & 0.0737 & 0.042 & 0.0061 & 0.3665 & 0.0153 \\
\hline 0.4507 & 0.0713 & 0.042 & 0.0073 & 0.4137 & 0.015 \\
\hline 0.4297 & 0.0832 & 0.0607 & 0.0082 & 0.397 & 0.0212 \\
\hline 0.4232 & 0.0835 & 0.0577 & 0.0079 & 0.4071 & 0.0206 \\
\hline 0.3176 & 0.0607 & 0.0439 & 0.0161 & 0.5458 & 0.016 \\
\hline 0.3451 & 0.0751 & .0692 & .0088 & 0.4698 & 0.0321 \\
\hline
\end{tabular}


Modern Calibration Dataset for OPTiMAL

\begin{tabular}{|c|c|c|c|c|c|}
\hline GDGT-0 & GDGT-1 & GDGT-2 & GDGT-3 & Zrenarchaeo & Cren' \\
\hline 0.3368 & 0.0779 & 0.0718 & 0.0091 & 0.4736 & 0.0308 \\
\hline 0.3357 & 0.0793 & 0.0685 & 0.0101 & 0.477 & 0.0293 \\
\hline 0.3142 & 0.0702 & 0.0583 & 0.0124 & 0.5157 & 0.0291 \\
\hline 0.3251 & 0.0702 & 0.0576 & 0.014 & 0.5073 & 0.0258 \\
\hline 0.3135 & 0.0754 & 0.0637 & 0.011 & 0.5069 & 0.0294 \\
\hline 0.3131 & 0.0684 & 0.0579 & 0.0144 & 0.5216 & 0.0246 \\
\hline 0.3329 & 0.0805 & 0.0738 & 0.0111 & 0.4726 & 0.0291 \\
\hline 0.3381 & 0.0776 & 0.0738 & 0.0099 & 0.4704 & 0.0302 \\
\hline 0.3225 & 0.0798 & 0.0785 & 0.0074 & 0.4752 & 0.0366 \\
\hline 0.3296 & 0.0797 & 0.0796 & 0.0085 & 0.4682 & 0.0344 \\
\hline 0.2958 & 0.0721 & 0.072 & 0.0135 & 0.5176 & 0.0289 \\
\hline 0.3009 & 0.0741 & 0.0743 & 0.0124 & 0.5081 & 0.0302 \\
\hline 0.4317 & 0.0237 & 0.0086 & 0.0037 & 0.5291 & 0.0032 \\
\hline 0.5356 & 0.0273 & 0.0113 & 0.0038 & 0.4192 & 0.0028 \\
\hline 0.4967 & 0.0204 & 0.0049 & 0.0025 & 0.4724 & 0.003 \\
\hline 0.5002 & 0.0145 & 0.0045 & 0.0026 & 0.4763 & 0.0019 \\
\hline 0.4966 & 0.0389 & 0.0141 & 0.0031 & 0.4427 & 0.0046 \\
\hline 0.2286 & 0.0575 & 0.0696 & 0.01 & 0.592 & 0.0424 \\
\hline 0.411 & 0.0211 & 0.0129 & 0.0074 & 0.5408 & 0.0067 \\
\hline 0.4342 & 0.0204 & 0.0108 & 0.0066 & 0.5223 & 0.0058 \\
\hline 0.4585 & 0.0279 & 0.0099 & 0.0061 & 0.4928 & 0.0049 \\
\hline 0.4927 & 0.0209 & 0.0064 & 0.0047 & 0.4715 & 0.0038 \\
\hline 0.485 & 0.0217 & 0.0062 & 0.0045 & 0.4782 & 0.0043 \\
\hline 0.5038 & 0.021 & 0.005 & 0.0045 & 0.4619 & 0.0038 \\
\hline 0.5333 & 0.0235 & 0.0113 & 0.0054 & 0.4217 & 0.0049 \\
\hline 0.5001 & 0.0218 & 0.0057 & 0.0042 & 0.4641 & 0.0041 \\
\hline 0.4486 & 0.0225 & 0.0088 & 0.0053 & 0.5105 & 0.0044 \\
\hline 0.4491 & 0.018 & 0.0052 & 0.0044 & 0.5183 & 0.0049 \\
\hline 0.4537 & 0.0191 & 0.0055 & 0.0045 & 0.5133 & 0.0038 \\
\hline 0.4853 & 0.0203 & 0.0072 & 0.0043 & 0.4789 & 0.004 \\
\hline 0.4966 & 0.0462 & 0.0365 & 0.019 & 0.391 & 0.0107 \\
\hline 0.4731 & 0.0348 & 0.0166 & 0.012 & 0.4559 & 0.0076 \\
\hline 0.4658 & 0.0428 & 0.0191 & 0.0154 & 0.449 & 0.0079 \\
\hline 0.5291 & 0.0357 & 0.0167 & 0.0102 & 0.4019 & 0.0063 \\
\hline 0.3757 & 0.0686 & 0.0575 & 0.0089 & 0.4677 & 0.0216 \\
\hline 0.3909 & 0.0662 & 0.0486 & 0.0098 & 0.4662 & 0.0184 \\
\hline 0.4116 & 0.0678 & 0.0427 & 0.0106 & 0.4525 & 0.0147 \\
\hline 0.4635 & 0.0373 & 0.0127 & 0.0049 & 0.4779 & 0.0037 \\
\hline 0.4588 & 0.0345 & 0.0103 & 0.0038 & 0.4891 & 0.0036 \\
\hline 0.4886 & 0.0279 & 0.0087 & 0.0037 & 0.4686 & 0.0025 \\
\hline 0.384 & 0.059 & 0.047 & 0.004 & 0.494 & 0.013 \\
\hline 0.388 & 0.056 & 0.043 & 0.003 & 0.494 & 0.015 \\
\hline 0.251 & 0.051 & 0.063 & 0.006 & 0.586 & 0.042 \\
\hline 0.365 & 0.058 & 0.051 & 0.003 & 0.498 & 0.024 \\
\hline 0.34 & 0.063 & 0.059 & 0.007 & 0.506 & 0.026 \\
\hline 0.345 & 0.067 & 0.063 & 0.006 & 0.49 & 0.031 \\
\hline 0.34 & 0.062 & 0.061 & 0.005 & 0.503 & 0.029 \\
\hline 0.224 & 0.06 & 0.066 & 0.007 & 0.598 & 0.045 \\
\hline 0.342 & 0.066 & 0.059 & 0.006 & 0.499 & 0.029 \\
\hline 0.359 & 0.068 & 0.062 & 0.004 & 0.48 & 0.026 \\
\hline 0.369 & 0.062 & 0.055 & 0.006 & 0.482 & 0.027 \\
\hline 0.344 & 0.071 & 0.069 & 0.004 & 0.481 & 0.032 \\
\hline 0.23 & 0.045 & 0.067 & 0.007 & 0.603 & 0.047 \\
\hline 0.548 & 0.041 & 0.012 & 0.002 & 0.392 & 0.005 \\
\hline 0.543 & 0.031 & 0.009 & 0.002 & 0.41 & 0.005 \\
\hline 0.54 & 0.042 & 0.014 & 0.002 & 0.396 & 0.007 \\
\hline 0.432 & 0.046 & 0.025 & 0.003 & 0.484 & 0.011 \\
\hline 0.452 & 0.049 & 0.025 & 0.002 & 0.462 & 0.009 \\
\hline 0.426 & 0.053 & 0.028 & 0.003 & 0.479 & 0.011 \\
\hline 0.419 & 0.055 & 0.033 & 0.003 & 0.479 & 0.011 \\
\hline 0.4703 & 0.012 & 0.004 & 0.0027 & 0.5084 & 0.0026 \\
\hline 0.4576 & 0.0125 & 0.0041 & 0.0029 & 0.5203 & 0.0026 \\
\hline 0.5037 & 0.0126 & 0.0036 & 0.0022 & 0.4754 & 0.0025 \\
\hline 0.5221 & 0.0248 & 0.0067 & 0.0019 & 0.4411 & 0.0034 \\
\hline 0.5262 & 0.0368 & 0.013 & 0.0039 & 0.4145 & 0.0056 \\
\hline 0.5312 & 0.0328 & 0.0107 & 0.0035 & 0.4165 & 0.0052 \\
\hline 0.5303 & 0.0337 & 0.0109 & 0.0036 & 0.4167 & 0.0048 \\
\hline 0.4821 & 0.0394 & 0.0176 & 0.0041 & 0.4511 & 0.0056 \\
\hline 0.5328 & 0.0367 & 0.0108 & 0.0039 & 0.4104 & 0.0053 \\
\hline 0.2393 & 0.0721 & 0.0885 & 0.0128 & 0.5367 & 0.0506 \\
\hline 0.2369 & 0.0699 & 0.0854 & 0.012 & 0.5491 & 0.0468 \\
\hline 0.2264 & 0.0747 & 0.0959 & 0.0125 & 0.537 & 0.0534 \\
\hline 0.5283 & 0.0365 & 0.0133 & 0.0051 & 0.4123 & 0.0045 \\
\hline 0.5824 & 0.0179 & 0.006 & 0.0034 & 0.3882 & 0.0021 \\
\hline 0.5319 & 0.0361 & 0.0116 & 0.0027 & 0.411 & 0.0068 \\
\hline 0.565 & 0.0373 & 0.0123 & 0.0025 & 0.376 & 0.0068 \\
\hline 0.1637 & 0.0535 & 0.0787 & 0.0142 & 0.6414 & 0.0485 \\
\hline 0.2178 & 0.0587 & 0.0804 & 0.0098 & 0.5938 & 0.0395 \\
\hline 0.2218 & 0.0617 & 0.0802 & 0.0115 & 0.5795 & 0.0453 \\
\hline 0.2101 & 0.0603 & 0.0768 & 0.0116 & 0.5949 & 0.0464 \\
\hline 0.1999 & 0.0616 & 0.0812 & 0.0109 & 0.5964 & 0.0501 \\
\hline 0.217 & 0.0631 & 0.0781 & 0.0121 & 0.5811 & 0.0487 \\
\hline 0.2086 & 0.0621 & 0.0772 & 0.0124 & 0.5913 & 0.0484 \\
\hline 0.5122 & 0.0476 & 0.0209 & 0.0099 & 0.4059 & 0.0036 \\
\hline 0.226 & 0.0658 & 0.0848 & 0.013 & 0.5469 & 0.0635 \\
\hline 0.2251 & 0.066 & 0.0816 & 0.0125 & 0.5547 & 0.06 \\
\hline 0.2314 & 0.0633 & 0.082 & 0.0125 & 0.5509 & 0.06 \\
\hline 0.2393 & 0.0656 & 0.0806 & 0.0119 & 0.5432 & 0.0593 \\
\hline 0.2276 & 0.0662 & 0.0809 & 0.0116 & 0.5541 & 0.0596 \\
\hline 0.2398 & 0.0678 & 0.084 & 0.0125 & 0.5334 & 0.0626 \\
\hline 0.2441 & 0.0681 & 0.0826 & 0.0128 & 0.5326 & 0.0598 \\
\hline 0.2341 & .06 & .08 & 013 & 54 & 0.06 \\
\hline
\end{tabular}


Modern Calibration Dataset for OPTiMAL

\begin{tabular}{|c|c|c|c|c|c|}
\hline GDGT-0 & GDGT-1 & GDGT-2 & GDGT-3 & Zrenarchaeo & Cren' \\
\hline 0.2248 & 0.0656 & 0.0802 & 0.012 & 0.5547 & 0.0627 \\
\hline 0.2802 & 0.0634 & 0.0791 & 0.0123 & 0.5097 & 0.0553 \\
\hline 0.2411 & 0.0633 & 0.0804 & 0.0121 & 0.5427 & 0.0604 \\
\hline 0.2348 & 0.0652 & 0.077 & 0.0117 & 0.5485 & 0.0627 \\
\hline 0.2508 & 0.0671 & 0.0871 & 0.0117 & 0.5258 & 0.0576 \\
\hline 0.2557 & 0.0638 & 0.076 & 0.0115 & 0.5319 & 0.0611 \\
\hline 0.2441 & 0.0678 & 0.0861 & 0.0125 & 0.533 & 0.0564 \\
\hline 0.5002 & 0.0137 & 0.0038 & 0.002 & 0.4774 & 0.0028 \\
\hline 0.4931 & 0.0146 & 0.0044 & 0.0021 & 0.4821 & 0.0037 \\
\hline 0.502 & 0.0131 & 0.0037 & 0.002 & 0.4784 & 0.0008 \\
\hline 0.5082 & 0.0122 & 0.0034 & 0.002 & 0.4719 & 0.0023 \\
\hline 0.541 & 0.0234 & 0.0068 & 0.0024 & 0.4237 & 0.0027 \\
\hline 0.4461 & 0.0237 & 0.0067 & 0.0023 & 0.5167 & 0.0045 \\
\hline 0.5435 & 0.017 & 0.0047 & 0.0021 & 0.4302 & 0.0025 \\
\hline 0.4704 & 0.0121 & 0.0041 & 0.0026 & 0.5081 & 0.0027 \\
\hline 0.5173 & 0.0162 & 0.0042 & 0.0017 & 0.4579 & 0.0028 \\
\hline 0.5208 & 0.015 & 0.004 & 0.0018 & 0.4555 & 0.0029 \\
\hline 0.5152 & 0.0206 & 0.0057 & 0.003 & 0.4527 & 0.0029 \\
\hline 0.5056 & 0.0232 & 0.0064 & 0.0027 & 0.459 & 0.0032 \\
\hline 0.531 & 0.0204 & 0.0056 & 0.0021 & 0.4378 & 0.0031 \\
\hline 0.5164 & 0.0328 & 0.0097 & 0.0027 & 0.4341 & 0.0043 \\
\hline 0.4778 & 0.0345 & 0.0113 & 0.003 & 0.4683 & 0.005 \\
\hline 0.5414 & 0.0219 & 0.0068 & 0.0024 & 0.4248 & 0.0026 \\
\hline 0.4367 & 0.013 & 0.0038 & 0.0017 & 0.542 & 0.0027 \\
\hline 0.4943 & 0.0148 & 0.0047 & 0.0024 & 0.4811 & 0.0028 \\
\hline 0.5227 & 0.0161 & 0.005 & 0.0025 & 0.4512 & 0.0025 \\
\hline 0.2791 & 0.0729 & 0.0924 & 0.0108 & 0.4953 & 0.0495 \\
\hline 0.2249 & 0.062 & 0.0614 & 0.0165 & 0.5924 & 0.0428 \\
\hline 0.2229 & 0.0675 & 0.0794 & 0.0166 & 0.5636 & 0.0501 \\
\hline 0.2319 & 0.0693 & 0.0761 & 0.0138 & 0.5611 & 0.0477 \\
\hline 0.2466 & 0.0682 & 0.0852 & 0.0155 & 0.5313 & 0.0531 \\
\hline 0.2443 & 0.0636 & 0.077 & 0.012 & 0.5429 & 0.0601 \\
\hline 0.2607 & 0.0649 & 0.0798 & 0.0128 & 0.5285 & 0.0533 \\
\hline 0.2863 & 0.0715 & 0.0842 & 0.0127 & 0.4941 & 0.0513 \\
\hline 0.2824 & 0.0717 & 0.0879 & 0.0121 & 0.4919 & 0.0541 \\
\hline 0.1955 & 0.0599 & 0.072 & 0.0139 & 0.5988 & 0.0599 \\
\hline 0.5059 & 0.0345 & 0.0131 & 0.0048 & 0.4381 & 0.0036 \\
\hline 0.4563 & 0.0532 & 0.0188 & 0.0071 & 0.4605 & 0.0041 \\
\hline 0.4572 & 0.0445 & 0.0196 & 0.0071 & 0.4676 & 0.0041 \\
\hline 0.4792 & 0.0495 & 0.0167 & 0.0057 & 0.4436 & 0.0051 \\
\hline 0.4896 & 0.014 & 0.0048 & 0.0031 & 0.486 & 0.0025 \\
\hline 0.4771 & 0.0132 & 0.0049 & 0.0031 & 0.4989 & 0.0028 \\
\hline 0.4563 & 0.0117 & 0.0043 & 0.0031 & 0.5225 & 0.002 \\
\hline 0.5652 & 0.0147 & 0.0048 & 0.0029 & 0.4109 & 0.0016 \\
\hline 0.5621 & 0.0175 & 0.0065 & 0.004 & 0.4073 & 0.0026 \\
\hline 0.5789 & 0.0182 & 0.0056 & 0.0031 & 0.3921 & 0.0022 \\
\hline 0.5814 & 0.0174 & 0.0054 & 0.003 & 0.3906 & 0.0022 \\
\hline 0.5641 & 0.0147 & 0.005 & 0.0031 & 0.4107 & 0.0023 \\
\hline 0.5511 & 0.0152 & 0.0051 & 0.0031 & 0.4231 & 0.0023 \\
\hline 0.4911 & 0.0133 & 0.0047 & 0.0031 & 0.486 & 0.0018 \\
\hline 0.4687 & 0.0123 & 0.0044 & 0.0031 & 0.5095 & 0.002 \\
\hline 0.483 & 0.0134 & 0.0047 & 0.0031 & 0.4936 & 0.0022 \\
\hline 0.4784 & 0.013 & 0.0046 & 0.0032 & 0.4983 & 0.0024 \\
\hline 0.5182 & 0.0161 & 0.0053 & 0.003 & 0.455 & 0.0023 \\
\hline 0.5301 & 0.0157 & 0.0051 & 0.0028 & 0.4444 & 0.0019 \\
\hline 0.5033 & 0.015 & 0.0051 & 0.0032 & 0.4711 & 0.0024 \\
\hline 0.5115 & 0.0149 & 0.0052 & 0.0031 & 0.463 & 0.0024 \\
\hline 0.5132 & 0.0148 & 0.0051 & 0.0032 & 0.4612 & 0.0025 \\
\hline 0.5524 & 0.0154 & 0.005 & 0.0031 & 0.4221 & 0.0021 \\
\hline 0.5478 & 0.0163 & 0.0055 & 0.0033 & 0.425 & 0.0021 \\
\hline 0.5492 & 0.0151 & 0.0052 & 0.0031 & 0.4256 & 0.0018 \\
\hline 0.5462 & 0.0146 & 0.005 & 0.003 & 0.4295 & 0.0018 \\
\hline 0.5433 & 0.0153 & 0.0049 & 0.0031 & 0.4316 & 0.0018 \\
\hline 0.5475 & 0.015 & 0.005 & 0.0031 & 0.4278 & 0.0016 \\
\hline 0.5304 & 0.0149 & 0.0049 & 0.0031 & 0.4447 & 0.0019 \\
\hline 0.5327 & 0.0159 & 0.0052 & 0.0031 & 0.441 & 0.0022 \\
\hline 0.4924 & 0.0129 & 0.0045 & 0.003 & 0.4852 & 0.0019 \\
\hline 0.4763 & 0.0124 & 0.0045 & 0.003 & 0.5017 & 0.0021 \\
\hline 0.4888 & 0.013 & 0.0044 & 0.0029 & 0.4888 & 0.0021 \\
\hline 0.5015 & 0.0136 & 0.0051 & 0.0032 & 0.4749 & 0.0018 \\
\hline 0.3788 & 0.0113 & 0.004 & 0.0028 & 0.6 & 0.0031 \\
\hline 0.5358 & 0.0152 & 0.0052 & 0.0031 & 0.4387 & 0.002 \\
\hline 0.5767 & 0.0147 & 0.0047 & 0.0027 & 0.3996 & 0.0016 \\
\hline 0.551 & 0.0151 & 0.0051 & 0.0033 & 0.424 & 0.0015 \\
\hline 0.5472 & 0.0171 & 0.0088 & 0.0045 & 0.4198 & 0.0025 \\
\hline 0.5382 & 0.0145 & 0.0047 & 0.0029 & 0.4376 & 0.002 \\
\hline 0.1854 & 0.0667 & 0.0771 & 0.0376 & 0.6004 & 0.0328 \\
\hline 0.307 & 0.0673 & 0.0593 & 0.0132 & 0.5256 & 0.0277 \\
\hline 0.2896 & 0.0697 & 0.0772 & 0.0115 & 0.5157 & 0.0362 \\
\hline 0.296 & 0.067 & 0.059 & 0.0139 & 0.5362 & 0.0279 \\
\hline 0.2766 & 0.073 & 0.0808 & 0.0115 & 0.5211 & 0.0369 \\
\hline 0.2826 & 0.0682 & 0.0747 & 0.0105 & 0.5272 & 0.0367 \\
\hline 0.3345 & 0.0648 & 0.0555 & 0.01 & 0.5068 & 0.0283 \\
\hline 0.316 & 0.0654 & 0.0621 & 0.0095 & 0.5159 & 0.0311 \\
\hline 0.276 & 0.0711 & 0.0771 & 0.0091 & 0.5272 & 0.0395 \\
\hline 0.3288 & 0.066 & 0.0639 & 0.0095 & 0.5003 & 0.0314 \\
\hline 0.2893 & 0.0723 & 0.0823 & 0.0086 & 0.5076 & 0.0399 \\
\hline 0.2982 & 0.0762 & 0.077 & 0.0084 & 0.5024 & 0.0379 \\
\hline 0.3084 & 0.0724 & 0.0697 & 0.0085 & 0.5036 & 0.0374 \\
\hline 0.3149 & 0.073 & 0.0723 & 0.0078 & 0.4955 & 0.0366 \\
\hline 0.2778 & 0.0654 & 0.076 & 0.0091 & 0.5311 & 0.0407 \\
\hline 0.3181 & .07 & .06 & 00 & 0.4975 & 0.0363 \\
\hline
\end{tabular}


Modern Calibration Dataset for OPTiMAL

\begin{tabular}{|c|c|c|c|c|c|}
\hline GDGT-0 & GDGT-1 & GDGT-2 & GDGT-3 & Zrenarchaeo & Cren' \\
\hline 0.3278 & 0.0682 & 0.0565 & 0.0084 & 0.5055 & 0.0335 \\
\hline 0.3331 & 0.0643 & 0.0504 & 0.0094 & 0.5123 & 0.0304 \\
\hline 0.3426 & 0.0701 & 0.0614 & 0.0093 & 0.4837 & 0.0329 \\
\hline 0.3315 & 0.0699 & 0.0565 & 0.0101 & 0.4994 & 0.0325 \\
\hline 0.3279 & 0.0721 & 0.0593 & 0.0095 & 0.4982 & 0.0329 \\
\hline 0.3401 & 0.0727 & 0.0639 & 0.0097 & 0.48 & 0.0336 \\
\hline 0.2814 & 0.0726 & 0.0794 & 0.0103 & 0.5158 & 0.0405 \\
\hline 0.2878 & 0.063 & 0.0749 & 0.0098 & 0.526 & 0.0384 \\
\hline 0.3804 & 0.0671 & 0.0484 & 0.0114 & 0.4737 & 0.019 \\
\hline 0.4117 & 0.0592 & 0.0357 & 0.0102 & 0.4663 & 0.0168 \\
\hline 0.5028 & 0.0331 & 0.0123 & 0.0049 & 0.4438 & 0.0032 \\
\hline 0.5256 & 0.0161 & 0.0057 & 0.0025 & 0.4474 & 0.0026 \\
\hline 0.5165 & 0.0192 & 0.0061 & 0.0025 & 0.4524 & 0.0033 \\
\hline 0.5426 & 0.0207 & 0.0062 & 0.0024 & 0.4246 & 0.0035 \\
\hline 0.5472 & 0.0195 & 0.006 & 0.0023 & 0.4226 & 0.0025 \\
\hline 0.3144 & 0.0481 & 0.0326 & 0.0102 & 0.5806 & 0.014 \\
\hline 0.2854 & 0.0516 & 0.0496 & 0.0133 & 0.5812 & 0.0189 \\
\hline 0.496 & 0.0225 & 0.0055 & 0.0026 & 0.4707 & 0.0027 \\
\hline 0.494 & 0.0554 & 0.0175 & 0.0029 & 0.4217 & 0.0085 \\
\hline 0.4962 & 0.0609 & 0.0179 & 0.0027 & 0.4146 & 0.0077 \\
\hline 0.497 & 0.0481 & 0.014 & 0.0031 & 0.4307 & 0.0071 \\
\hline 0.5214 & 0.0371 & 0.0121 & 0.0043 & 0.4194 & 0.0057 \\
\hline 0.503 & 0.0342 & 0.0099 & 0.003 & 0.4446 & 0.0052 \\
\hline 0.49 & 0.0383 & 0.0152 & 0.0038 & 0.4473 & 0.0053 \\
\hline 0.5049 & 0.0218 & 0.0058 & 0.006 & 0.4578 & 0.0037 \\
\hline 0.5506 & 0.0245 & 0.0062 & 0.0059 & 0.4103 & 0.0025 \\
\hline 0.5336 & 0.0275 & 0.0078 & 0.0035 & 0.4238 & 0.0038 \\
\hline 0.5053 & 0.0248 & 0.0066 & 0.0035 & 0.4559 & 0.0039 \\
\hline 0.5111 & 0.0208 & 0.0052 & 0.0025 & 0.4566 & 0.0038 \\
\hline 0.541 & 0.0527 & 0.0221 & 0.0033 & 0.371 & 0.0099 \\
\hline 0.5614 & 0.0533 & 0.0226 & 0 & 0.3539 & 0.0089 \\
\hline 0.5265 & 0.0611 & 0.0232 & 0.0046 & 0.372 & 0.0126 \\
\hline 0.4988 & 0.0659 & 0.0259 & 0.0047 & 0.3917 & 0.0131 \\
\hline 0.4952 & 0.0242 & 0.0255 & 0.0067 & 0.4423 & 0.0061 \\
\hline 0.4942 & 0.0415 & 0.0128 & 0.0063 & 0.4388 & 0.0063 \\
\hline 0.4911 & 0.0238 & 0.0136 & 0.0046 & 0.4626 & 0.0044 \\
\hline 0.4878 & 0.0214 & 0.0152 & 0.0047 & 0.4671 & 0.0036 \\
\hline 0.4756 & 0.022 & 0.0179 & 0.0058 & 0.4735 & 0.0052 \\
\hline 0.4903 & 0.0409 & 0.0164 & 0.0056 & 0.4401 & 0.0067 \\
\hline 0.4891 & 0.0306 & 0.0118 & 0.0043 & 0.4591 & 0.0051 \\
\hline 0.5031 & 0.0272 & 0.016 & 0.0044 & 0.4444 & 0.0048 \\
\hline 0.4938 & 0.0266 & 0.0137 & 0.004 & 0.4569 & 0.005 \\
\hline 0.5034 & 0.02 & 0.0119 & 0.004 & 0.4566 & 0.004 \\
\hline 0.4497 & 0.068 & 0.044 & 0.0037 & 0.4187 & 0.0159 \\
\hline 0.6066 & 0.0459 & 0.0149 & 0.0025 & 0.3221 & 0.0079 \\
\hline 0.5795 & 0.0552 & 0.0178 & 0.0029 & 0.3372 & 0.0075 \\
\hline 0.5006 & 0.0674 & 0.0389 & 0.0016 & 0.3815 & 0.0101 \\
\hline 0.5546 & 0.0547 & 0.0277 & 0.0022 & 0.3541 & 0.0067 \\
\hline 0.5462 & 0.0565 & 0.0321 & 0.0024 & 0.3518 & 0.0109 \\
\hline 0.5339 & 0.0659 & 0.0216 & 0.0011 & 0.3713 & 0.0061 \\
\hline 0.4546 & 0.0795 & 0.0335 & 0.0038 & 0.418 & 0.0106 \\
\hline 0.5447 & 0.0662 & 0.0277 & 0.0017 & 0.3526 & 0.007 \\
\hline 0.5028 & 0.0233 & 0.0324 & 0.0067 & 0.4295 & 0.0053 \\
\hline 0.5256 & 0.0292 & 0.0132 & 0.0045 & 0.4223 & 0.0052 \\
\hline 0.5121 & 0.0331 & 0.0121 & 0.0043 & 0.4329 & 0.0056 \\
\hline 0.5794 & 0.0538 & 0.0193 & 0.0023 & 0.3387 & 0.0064 \\
\hline 0.577 & 0.057 & 0.0188 & 0.0036 & 0.3356 & 0.008 \\
\hline 0.5816 & 0.0496 & 0.0156 & 0.0029 & 0.3428 & 0.0074 \\
\hline 0.5585 & 0.0169 & 0.006 & 0.0042 & 0.3825 & 0.032 \\
\hline 0.5603 & 0.0169 & 0.0058 & 0.0027 & 0.3815 & 0.0328 \\
\hline 0.5425 & 0.0598 & 0.0221 & 0.0054 & 0.348 & 0.0221 \\
\hline 0.581 & 0.0323 & 0.01 & 0.0026 & 0.3443 & 0.0299 \\
\hline 0.5818 & 0.0341 & 0.0107 & 0.0034 & 0.3468 & 0.0232 \\
\hline 0.5675 & 0.0315 & 0.0098 & 0.0034 & 0.3642 & 0.0236 \\
\hline 0.5649 & 0.0353 & 0.0111 & 0.003 & 0.3504 & 0.0353 \\
\hline 0.5805 & 0.0273 & 0.0091 & 0.0029 & 0.3557 & 0.0245 \\
\hline 0.5846 & 0.0301 & 0.0087 & 0.0036 & 0.3401 & 0.0329 \\
\hline 0.5744 & 0.0381 & 0.0147 & 0.0033 & 0.3457 & 0.0239 \\
\hline 0.6003 & 0.0331 & 0.0113 & 0.0037 & 0.331 & 0.0207 \\
\hline 0.612 & 0.0301 & 0.0102 & 0.004 & 0.3116 & 0.0322 \\
\hline 0.6201 & 0.0226 & 0.0063 & 0.0022 & 0.331 & 0.0178 \\
\hline 0.5652 & 0.0214 & 0.0061 & 0.0034 & 0.375 & 0.0289 \\
\hline 0.5504 & 0.0386 & 0.0135 & 0.0038 & 0.3704 & 0.0233 \\
\hline 0.4931 & 0.0159 & 0.0041 & 0.0024 & 0.4829 & 0.0016 \\
\hline 0.4912 & 0.0307 & 0.0079 & 0 & 0.4702 & 0 \\
\hline 0.5653 & 0.0358 & 0.0109 & 0.0023 & 0.3858 & 0 \\
\hline 0.5226 & 0.0407 & 0.0128 & 0.0027 & 0.4175 & 0.0037 \\
\hline 0.5127 & 0.0618 & 0.0215 & 0.0033 & 0.3955 & 0.0053 \\
\hline 0.5191 & 0.0537 & 0.0177 & 0.003 & 0.4002 & 0.0064 \\
\hline 0.4523 & 0.0672 & 0.0281 & 0.0026 & 0.4421 & 0.0078 \\
\hline 0.5263 & 0.0342 & 0.0109 & 0.0037 & 0.4215 & 0.0034 \\
\hline 0.5288 & 0.0255 & 0.0105 & 0.0037 & 0.4163 & 0.0154 \\
\hline 0.5246 & 0.0443 & 0.0155 & 0.0054 & 0.3868 & 0.0234 \\
\hline 0.5409 & 0.0465 & 0.0142 & 0.0022 & 0.3658 & 0.0303 \\
\hline 0.5446 & 0.062 & 0.0172 & 0.0018 & 0.345 & 0.0294 \\
\hline 0.5656 & 0.0531 & 0.0234 & 0.0011 & 0.3304 & 0.0264 \\
\hline 0.486 & 0.0703 & 0.0353 & 0.0032 & 0.3995 & 0.0056 \\
\hline 0.4717 & 0.0627 & 0.0338 & 0.0033 & 0.4134 & 0.0151 \\
\hline 0.5165 & 0.0692 & 0.0309 & 0.0038 & 0.3682 & 0.0115 \\
\hline 0.5482 & 0.0736 & 0.0295 & 0.0028 & 0.3362 & 0.0096 \\
\hline 0.548 & 0.0604 & 0.0248 & 0.0028 & 0.3503 & 0.0137 \\
\hline 0. & 06 & 01 & 00 & 0.322 & 0.00 \\
\hline
\end{tabular}




\begin{tabular}{|c|c|c|c|c|c|}
\hline GDGT-0 & GDGT-1 & GDGT-2 & GDGT-3 & renarchaeo & Cren' \\
\hline 0.5682 & 0.0462 & 0.0178 & 0.0019 & 0.3573 & 0.0086 \\
\hline 0.5807 & 0.0632 & 0.0193 & 0.0023 & 0.3232 & 0.0114 \\
\hline 0.5516 & 0.0614 & 0.0226 & 0.0028 & 0.3524 & 0.0092 \\
\hline 0.563 & 0.0672 & 0.026 & 0.0035 & 0.3307 & 0.0097 \\
\hline 0.5296 & 0.0703 & 0.0301 & 0.0027 & 0.3522 & 0.0152 \\
\hline 0.4954 & 0.0719 & 0.0347 & 0.0029 & 0.3845 & 0.0105 \\
\hline 0.5449 & 0.0641 & 0.0272 & 0.0027 & 0.3531 & 0.008 \\
\hline 0.5387 & 0.0712 & 0.03 & 0.0033 & 0.3479 & 0.0088 \\
\hline 0.5464 & 0.0592 & 0.0268 & 0.0034 & 0.357 & 0.0072 \\
\hline 0.5371 & 0.0638 & 0.0264 & 0.0029 & 0.3599 & 0.0099 \\
\hline 0.5915 & 0.0545 & 0.0194 & 0.0023 & 0.3258 & 0.0064 \\
\hline 0.5809 & 0.0484 & 0.0175 & 0.0026 & 0.3421 & 0.0085 \\
\hline 0.5497 & 0.0502 & 0.019 & 0.0028 & 0.3677 & 0.0106 \\
\hline 0.5576 & 0.0562 & 0.018 & 0.0027 & 0.3541 & 0.0114 \\
\hline 0.5626 & 0.0578 & 0.0204 & 0.0025 & 0.3483 & 0.0085 \\
\hline 0.5707 & 0.0684 & 0.0246 & 0.003 & 0.323 & 0.0103 \\
\hline 0.5065 & 0.0798 & 0.0342 & 0.0028 & 0.3633 & 0.0133 \\
\hline 0.5406 & 0.0653 & 0.0271 & 0.0026 & 0.3547 & 0.0096 \\
\hline 0.516 & 0.0699 & 0.0316 & 0.0039 & 0.3681 & 0.0105 \\
\hline 0.5314 & 0.0673 & 0.0341 & 0.0027 & 0.3528 & 0.0116 \\
\hline 0.5074 & 0.0677 & 0.0349 & 0.0037 & 0.3712 & 0.0153 \\
\hline 0.5105 & 0.0639 & 0.0346 & 0.0045 & 0.3719 & 0.0147 \\
\hline 0.4991 & 0.0653 & 0.0364 & 0.0035 & 0.383 & 0.0127 \\
\hline 0.5046 & 0.0692 & 0.0316 & 0.0044 & 0.3769 & 0.0133 \\
\hline 0.5052 & 0.0582 & 0.029 & 0.0071 & 0.3917 & 0.0088 \\
\hline 0.5133 & 0.0502 & 0.0231 & 0.0066 & 0.3953 & 0.0115 \\
\hline 0.1013 & 0.0459 & 0.0906 & 0.0226 & 0.6541 & 0.0855 \\
\hline 0.1705 & 0.0584 & 0.0995 & 0.02 & 0.5709 & 0.0807 \\
\hline 0.0989 & 0.047 & 0.0962 & 0.0222 & 0.654 & 0.0816 \\
\hline 0.1231 & 0.0545 & 0.1113 & 0.0241 & 0.5947 & 0.0924 \\
\hline 0.1112 & 0.0477 & 0.0924 & 0.0213 & 0.6395 & 0.0878 \\
\hline 0.1137 & 0.0497 & 0.1137 & 0.022 & 0.6022 & 0.0988 \\
\hline 0.1061 & 0.0458 & 0.1216 & 0.021 & 0.6056 & 0.0999 \\
\hline 0.0828 & 0.0393 & 0.1143 & 0.0215 & 0.6269 & 0.1152 \\
\hline 0.0874 & 0.0402 & 0.1155 & 0.0205 & 0.6156 & 0.1207 \\
\hline 0.0771 & 0.0373 & 0.1111 & 0.0236 & 0.6423 & 0.1086 \\
\hline 0.0768 & 0.0327 & 0.1125 & 0.0226 & 0.6377 & 0.1177 \\
\hline 0.0795 & 0.0377 & 0.1249 & 0.0236 & 0.6174 & 0.1169 \\
\hline 0.0721 & 0.0368 & 0.1015 & 0.0208 & 0.6691 & 0.0996 \\
\hline 0.0841 & 0.0401 & 0.1228 & 0.0238 & 0.617 & 0.1122 \\
\hline 0.0749 & 0.0385 & 0.1079 & 0.0231 & 0.639 & 0.1167 \\
\hline 0.0781 & 0.0372 & 0.1074 & 0.0211 & 0.6565 & 0.0998 \\
\hline 0.0747 & 0.0367 & 0.1016 & 0.0194 & 0.6622 & 0.1054 \\
\hline 0.0808 & 0.0422 & 0.1028 & 0.0186 & 0.6564 & 0.0992 \\
\hline 0.0904 & 0.043 & 0.0983 & 0.0195 & 0.6583 & 0.0905 \\
\hline 0.1146 & 0.0457 & 0.0807 & 0.019 & 0.6577 & 0.0823 \\
\hline 0.182 & 0.05 & 0.064 & 0.011 & 0.635 & 0.058 \\
\hline 0.176 & 0.051 & 0.07 & 0.011 & 0.63 & 0.061 \\
\hline 0.152 & 0.046 & 0.068 & 0.016 & 0.66 & 0.059 \\
\hline 0.204 & 0.057 & 0.07 & 0.012 & 0.602 & 0.055 \\
\hline 0.181 & 0.048 & 0.065 & 0.012 & 0.642 & 0.051 \\
\hline 0.194 & 0.05 & 0.063 & 0.009 & 0.625 & 0.06 \\
\hline 0.18 & 0.049 & 0.072 & 0.009 & 0.628 & 0.061 \\
\hline 0.177 & 0.052 & 0.076 & 0.009 & 0.622 & 0.063 \\
\hline 0.4363 & 0.0824 & 0.0507 & 0.0021 & 0.4135 & 0.0151 \\
\hline 0.4892 & 0.0156 & 0.0052 & 0.003 & 0.4848 & 0.0021 \\
\hline 0.4824 & 0.073 & 0.0388 & 0.004 & 0.3894 & 0.0124 \\
\hline 0.4922 & 0.0798 & 0.0409 & 0.0035 & 0.3726 & 0.0109 \\
\hline 0.4359 & 0.082 & 0.0535 & 0 & 0.4286 & 0 \\
\hline 0.4306 & 0.0924 & 0.041 & 0 & 0.4222 & 0.0138 \\
\hline 0.4564 & 0.0873 & 0.0533 & 0.0037 & 0.3981 & 0.0013 \\
\hline 0.4448 & 0.0736 & 0.0462 & 0.0038 & 0.4182 & 0.0134 \\
\hline 0.4852 & 0.0735 & 0.0378 & 0.004 & 0.3981 & 0.0013 \\
\hline 0.463 & 0.0649 & 0.0407 & 0.0067 & 0.4089 & 0.0158 \\
\hline 0.4877 & 0.0559 & 0.0327 & 0.0037 & 0.4088 & 0.0112 \\
\hline 0.7588 & 0.1155 & 0.0583 & 0 & 0.0674 & 0 \\
\hline 0.4475 & 0.07 & 0.043 & 0.0033 & 0.4241 & 0.0121 \\
\hline 0.4682 & 0.0602 & 0.0337 & 0.0036 & 0.4242 & 0.0102 \\
\hline 0.447 & 0.0356 & 0.0165 & 0.003 & 0.4891 & 0.0088 \\
\hline 0.4577 & 0.0751 & 0.0421 & 0.0032 & 0.4101 & 0.0118 \\
\hline 0.4388 & 0.0687 & 0.028 & 0.0074 & 0.4461 & 0.0111 \\
\hline 0.4963 & 0.0556 & 0.0275 & 0.0036 & 0.4093 & 0.0078 \\
\hline 0.4385 & 0.0779 & 0.0555 & 0 & 0.4102 & 0.018 \\
\hline 0.4415 & 0.0714 & 0.0533 & 0 & 0.421 & 0.0128 \\
\hline 0.4447 & 0.0851 & 0.0521 & 0 & 0.4012 & 0.017 \\
\hline 0.4467 & 0.0702 & 0.0467 & 0 & 0.4205 & 0.016 \\
\hline 0.4279 & 0.0794 & 0.0516 & 0.004 & 0.4229 & 0.0142 \\
\hline 0.4391 & 0.0803 & 0.0512 & 0.0038 & 0.4121 & 0.0136 \\
\hline 0.7312 & 0.1237 & 0.0765 & 0.0057 & 0.0613 & 0.0018 \\
\hline 0.4857 & 0.0611 & 0.0373 & 0.0035 & 0.4023 & 0.01 \\
\hline 0.4465 & 0.0595 & 0.0288 & 0.003 & 0.4483 & 0.0139 \\
\hline 0.444 & 0.0657 & 0.0392 & 0.0045 & 0.4295 & 0.0171 \\
\hline 0.7234 & 0.1208 & 0.0704 & 0.0099 & 0.0728 & 0.0028 \\
\hline 0.7106 & 0.1187 & 0.0761 & 0 & 0.0904 & 0.0042 \\
\hline 0.4646 & 0.0621 & 0.0375 & 0 & 0.4102 & 0.0257 \\
\hline 0.4263 & 0.0665 & 0.0437 & 0 & 0.4439 & 0.0196 \\
\hline 0.4192 & 0.0646 & 0.0429 & 0.0064 & 0.4454 & 0.0214 \\
\hline 0.7518 & 0.1037 & 0.0667 & 0.0102 & 0.065 & 0.0027 \\
\hline 0.4224 & 0.0647 & 0.0428 & 0 & 0.4544 & 0.0157 \\
\hline 0.2 & 0.059 & 0.078 & 0.011 & 0.597 & 0.056 \\
\hline 0.169 & 0.051 & 0.063 & 0.014 & 0.651 & 0.053 \\
\hline & & 0 & 0 & & 0 \\
\hline
\end{tabular}


Modern Calibration Dataset for OPTiMAL

\begin{tabular}{|c|c|c|c|c|c|}
\hline GDGT-0 & GDGT-1 & GDGT-2 & GDGT-3 & :renarchaeo & Cren' \\
\hline 0.197 & 0.061 & 0.073 & 0.01 & 0.604 & 0.054 \\
\hline 0.193 & 0.066 & 0.07 & 0.009 & 0.613 & 0.048 \\
\hline 0.217 & 0.055 & 0.073 & 0.009 & 0.592 & 0.054 \\
\hline 0.2 & 0.063 & 0.086 & 0.012 & 0.575 & 0.065 \\
\hline 0.213 & 0.057 & 0.073 & 0.011 & 0.592 & 0.054 \\
\hline 0.203 & 0.064 & 0.087 & 0.011 & 0.573 & 0.06 \\
\hline 0.193 & 0.062 & 0.085 & 0.011 & 0.591 & 0.059 \\
\hline 0.183 & 0.057 & 0.077 & 0.01 & 0.625 & 0.049 \\
\hline 0.212 & 0.06 & 0.078 & 0.01 & 0.584 & 0.057 \\
\hline 0.205 & 0.061 & 0.078 & 0.012 & 0.586 & 0.058 \\
\hline 0.201 & 0.058 & 0.075 & 0.01 & 0.604 & 0.053 \\
\hline 0.213 & 0.062 & 0.085 & 0.01 & 0.571 & 0.059 \\
\hline 0.218 & 0.055 & 0.079 & 0.009 & 0.577 & 0.061 \\
\hline 0.199 & 0.052 & 0.076 & 0.009 & 0.606 & 0.058 \\
\hline 0.179 & 0.05 & 0.08 & 0.01 & 0.63 & 0.051 \\
\hline 0.179 & 0.052 & 0.077 & 0.01 & 0.633 & 0.049 \\
\hline 0.145 & 0.05 & 0.071 & 0.013 & 0.663 & 0.058 \\
\hline 0.135 & 0.05 & 0.077 & 0.014 & 0.667 & 0.058 \\
\hline 0.119 & 0.047 & 0.072 & 0.015 & 0.7 & 0.046 \\
\hline 0.137 & 0.049 & 0.07 & 0.013 & 0.67 & 0.06 \\
\hline 0.4781 & 0.043 & 0.0174 & 0.0068 & 0.4498 & 0.0049 \\
\hline 0.5112 & 0.0533 & 0.022 & 0.0038 & 0.3923 & 0.0174 \\
\hline 0.5417 & 0.0505 & 0.0176 & 0.003 & 0.3781 & 0.0092 \\
\hline 0.5119 & 0.0597 & 0.0233 & 0.0038 & 0.3865 & 0.0148 \\
\hline 0.5317 & 0.0235 & 0.0069 & 0.0027 & 0.4308 & 0.0044 \\
\hline 0.4826 & 0.0197 & 0.0051 & 0.0025 & 0.487 & 0.0031 \\
\hline 0.4794 & 0.0181 & 0.0048 & 0.0025 & 0.4917 & 0.0035 \\
\hline 0.175 & 0.05 & 0.069 & 0.012 & 0.64 & 0.054 \\
\hline 0.3429 & 0.0696 & 0.0654 & 0.0088 & 0.4751 & 0.0381 \\
\hline 0.3484 & 0.0695 & 0.0632 & 0.0035 & 0.4421 & 0.0733 \\
\hline 0.1884 & 0.0503 & 0.0652 & 0.0102 & 0.6468 & 0.0391 \\
\hline 0.2911 & 0.0759 & 0.0732 & 0.0142 & 0.5139 & 0.0318 \\
\hline 0.2987 & 0.0774 & 0.0816 & 0.0133 & 0.4918 & 0.0373 \\
\hline 0.2723 & 0.0743 & 0.0804 & 0.0173 & 0.5192 & 0.0363 \\
\hline 0.3191 & 0.0734 & 0.0738 & 0.0164 & 0.4724 & 0.0447 \\
\hline 0.3389 & 0.0778 & 0.0765 & 0.0126 & 0.4624 & 0.0318 \\
\hline 0.2737 & 0.0661 & 0.0746 & 0.0109 & 0.5372 & 0.0375 \\
\hline 0.2628 & 0.0708 & 0.0743 & 0.0158 & 0.5438 & 0.0324 \\
\hline 0.2571 & 0.074 & 0.0754 & 0.0137 & 0.5483 & 0.0315 \\
\hline 0.2758 & 0.0678 & 0.0733 & 0.014 & 0.5384 & 0.0307 \\
\hline 0.3241 & 0.0813 & 0.0848 & 0.01 & 0.4627 & 0.0371 \\
\hline 0.2383 & 0.0581 & 0.0735 & 0.0118 & 0.5901 & 0.0283 \\
\hline 0.1695 & 0.0709 & 0.0892 & 0.0113 & 0.6436 & 0.0157 \\
\hline 0.3931 & 0.0796 & 0.0613 & 0.0051 & 0.4273 & 0.0336 \\
\hline 0.335 & 0.0896 & 0.0627 & 0.0087 & 0.4717 & 0.0324 \\
\hline 0.3645 & 0.0895 & 0.0729 & 0.0126 & 0.4322 & 0.0284 \\
\hline 0.322 & 0.0759 & 0.0599 & 0.01 & 0.5052 & 0.0269 \\
\hline 0.3168 & 0.0642 & 0.0499 & 0.0097 & 0.534 & 0.0254 \\
\hline 0.3522 & 0.0556 & 0.0336 & 0.0111 & 0.5269 & 0.0206 \\
\hline 0.3533 & 0.0909 & 0.0732 & 0.0068 & 0.4476 & 0.0282 \\
\hline 0.3473 & 0.0911 & 0.0716 & 0.0058 & 0.4471 & 0.0371 \\
\hline 0.3528 & 0.0878 & 0.0719 & 0.0055 & 0.4494 & 0.0326 \\
\hline 0.3961 & 0.0619 & 0.0392 & 0.006 & 0.4718 & 0.0251 \\
\hline 0.4492 & 0.0709 & 0.0411 & 0.0055 & 0.4167 & 0.0166 \\
\hline 0.454 & 0.0675 & 0.04 & 0.0043 & 0.4194 & 0.0149 \\
\hline 0.5034 & 0.0266 & 0.0123 & 0.0042 & 0.4472 & 0.0063 \\
\hline 0.4653 & 0.0275 & 0.0153 & 0.0055 & 0.4781 & 0.0083 \\
\hline 0.472 & 0.0255 & 0.0109 & 0.0021 & 0.4842 & 0.0054 \\
\hline 0.4461 & 0.053 & 0.0288 & 0.0038 & 0.4565 & 0.0117 \\
\hline 0.4304 & 0.0617 & 0.0385 & 0.0038 & 0.451 & 0.0147 \\
\hline 0.2102 & 0.0669 & 0.0783 & 0.0125 & 0.5662 & 0.066 \\
\hline 0.6513 & 0.0659 & 0.0254 & 0.0041 & 0.2463 & 0.0071 \\
\hline 0.6548 & 0.0578 & 0.0219 & 0.0030 & 0.2554 & 0.0070 \\
\hline 0.5894 & 0.0736 & 0.0359 & 0.0033 & 0.2901 & 0.0077 \\
\hline 0.5899 & 0.0864 & 0.0405 & 0.0052 & 0.2695 & 0.0085 \\
\hline 0.6677 & 0.0668 & 0.0251 & 0.0036 & 0.2306 & 0.0063 \\
\hline 0.8165 & 0.0969 & 0.0407 & 0.0039 & 0.0324 & 0.0096 \\
\hline 0.7602 & 0.0296 & 0.0078 & 0.0030 & 0.1958 & 0.0035 \\
\hline 0.6775 & 0.0381 & 0.0125 & 0.0046 & 0.2617 & 0.0055 \\
\hline 0.6828 & 0.0394 & 0.0148 & 0.0030 & 0.2551 & 0.0050 \\
\hline 0.6728 & 0.0201 & 0.0061 & 0.0030 & 0.2924 & 0.0056 \\
\hline 0.1671 & 0.0456 & 0.0136 & 0.0115 & 0.7532 & 0.0090 \\
\hline 0.6624 & 0.0188 & 0.0065 & 0.0044 & 0.3038 & 0.0040 \\
\hline 0.6856 & 0.0207 & 0.0059 & 0.0044 & 0.2800 & 0.0034 \\
\hline 0.6515 & 0.0211 & 0.0069 & 0.0049 & 0.3124 & 0.0032 \\
\hline 0.6607 & 0.0585 & 0.0274 & 0.0035 & 0.2431 & 0.0067 \\
\hline 0.6888 & 0.0205 & 0.0067 & 0.0044 & 0.2758 & 0.0038 \\
\hline 0.6648 & 0.0217 & 0.0068 & 0.0040 & 0.2984 & 0.0044 \\
\hline 0.6352 & 0.0193 & 0.0064 & 0.0049 & 0.3300 & 0.0042 \\
\hline 0.6602 & 0.0158 & 0.0042 & 0.0030 & 0.3125 & 0.0042 \\
\hline 0.1593 & 0.0654 & 0.0196 & 0.0083 & 0.7371 & 0.0103 \\
\hline 0.6442 & 0.0209 & 0.0064 & 0.0039 & 0.3202 & 0.0043 \\
\hline 0.7043 & 0.0350 & 0.0118 & 0.0033 & 0.2402 & 0.0053 \\
\hline 0.6466 & 0.0742 & 0.0287 & 0.0031 & 0.2403 & 0.0071 \\
\hline 0.6843 & 0.0312 & 0.0113 & 0.0042 & 0.2631 & 0.0059 \\
\hline 0.6046 & 0.0831 & 0.0440 & 0.0035 & 0.2553 & 0.0094 \\
\hline 0.6583 & 0.0203 & 0.0073 & 0.0049 & 0.3047 & 0.0044 \\
\hline 0.7365 & 0.0220 & 0.0066 & 0.0038 & 0.2271 & 0.0039 \\
\hline 0.6551 & 0.0701 & 0.0284 & 0.0043 & 0.2335 & 0.0085 \\
\hline 0.6446 & 0.0698 & 0.0299 & 0.0036 & 0.2434 & 0.0086 \\
\hline 0.6476 & 0.0910 & 0.0377 & 0.0034 & 0.2109 & 0.0094 \\
\hline 0.6404 & 0.0818 & .0404 & .0031 & 0.2266 & 0.0077 \\
\hline
\end{tabular}


Modern Calibration Dataset for OPTiMAL

\begin{tabular}{|c|c|c|c|c|c|c|c|c|c|c|c|}
\hline GDGT-0 & GDGT-1 & GDGT-2 & GDGT-3 & :renarchaeo & Cren' & longitude & latitude & Sea Surface Temp & water depth & Core name & Source \\
\hline 0.6265 & 0.0590 & 0.0302 & 0.0038 & 0.2710 & 0.0096 & 150.074 & 53.276 & 4.7 & 1140 & YK07-12 St8 & Seki et al. 2044 \\
\hline 0.7289 & 0.0321 & 0.0089 & 0.0027 & 0.2225 & 0.0050 & 149.323 & 54.751 & 4.7 & 600 & YK07-12 St10 & Seki et al. 2045 \\
\hline 0.2378 & 0.0625 & 0.0824 & 0.0148 & 0.5533 & 0.0493 & 123.3 & 4.47 & 28.9 & 4095 & ODP767 & Seki et al. 2046 \\
\hline 0.1515 & 0.0491 & 0.0891 & 0.0155 & 0.6439 & 0.0510 & 119.421 & 16.391 & 28.3 & 1529 & ODP772 & Seki et al. 2047 \\
\hline 0.3011 & 0.0667 & 0.0660 & 0.0096 & 0.5230 & 0.0336 & 141.189 & 30.517 & 22.9 & 2958 & ODP782 & Seki et al. 2048 \\
\hline 0.2633 & 0.0553 & 0.0557 & 0.0106 & 0.5788 & 0.0364 & 140.533 & 31.635 & 22.7 & 2964 & ODP793 & Seki et al. 2049 \\
\hline 0.4809 & 0.0507 & 0.0233 & 0.0037 & 0.4323 & 0.0092 & 139.247 & 42.509 & 12 & 2570 & ODP796 & Seki et al. 2050 \\
\hline 0.4830 & 0.0331 & 0.0172 & 0.0063 & 0.4515 & 0.0090 & 133.52 & 39.132 & 13.4 & 2073 & ODP799 & Seki et al. 2051 \\
\hline 0.2908 & 0.0608 & 0.0655 & 0.0129 & 0.5331 & 0.0369 & 134.567 & 32.211 & 23.1 & 4676 & ODP808 & Seki et al. 2052 \\
\hline 0.2734 & 0.0662 & 0.0763 & 0.0128 & 0.5383 & 0.0330 & 172.207 & 5.334 & 28.8 & 1254 & ODP871 & Seki et al. 2053 \\
\hline 0.2581 & 0.0707 & 0.0817 & 0.0117 & 0.5350 & 0.0429 & 167.36 & 50.218 & 5.5 & 3244 & ODP882 & Seki et al. 2054 \\
\hline 0.4689 & 0.0680 & 0.0302 & 0.0031 & 0.4192 & 0.0106 & 167.4613 & 51.11898 & 5.5 & 3826 & ODP883 & Seki et al. 2055 \\
\hline 0.4305 & 0.0724 & 0.0343 & 0.0039 & 0.4460 & 0.0130 & 168.202 & 51.27 & 5.5 & 3826 & ODP884 & Seki et al. 2056 \\
\hline 0.4510 & 0.0567 & 0.0438 & 0.0031 & 0.4327 & 0.0128 & -148.268 & 54.219 & 7.2 & 3631 & ODP887 & Seki et al. 2057 \\
\hline 0.2480 & 0.0710 & 0.0906 & 0.0142 & 0.5206 & 0.0556 & 117.251 & 20.318 & 26.4 & 2035 & ODP1144 & Seki et al. 2058 \\
\hline 0.2346 & 0.0634 & 0.0816 & 0.0120 & 0.5533 & 0.0551 & 116.339 & 18.502 & 27 & 3297 & ODP1148 & Seki et al. 2059 \\
\hline 0.3514 & 0.0502 & 0.0530 & 0.0102 & 0.4996 & 0.0357 & 143.211 & 31.205 & 22.5 & 5817 & ODP1149 & Seki et al. 2060 \\
\hline 0.2448 & 0.0556 & 0.0581 & 0.0122 & 0.5940 & 0.0354 & 135.151 & 32.147 & 22.9 & 4790 & ODP1173 & Seki et al. 2061 \\
\hline 0.3779 & 0.0811 & 0.0603 & 0.0087 & 0.4507 & 0.0213 & 134.387 & 32.359 & 23.1 & 2997 & ODP1175 & Seki et al. 2062 \\
\hline 0.2878 & 0.0703 & 0.0738 & 0.0131 & 0.5134 & 0.0416 & 134.288 & 32.439 & 23.3 & 1741 & ODP1178 & Seki et al. 2063 \\
\hline 0.4647 & 0.0628 & 0.0273 & 0.0040 & 0.4284 & 0.0127 & 159.578 & 41.479 & 11.5 & 5565 & ODP1179 & Seki et al. 2064 \\
\hline 0.2949 & 0.0557 & 0.0676 & 0.0141 & 0.5288 & 0.0389 & 122.3 & 24.482 & 26.2 & 1274 & ODP1202 & Seki et al. 2065 \\
\hline 0.3564 & 0.0487 & 0.0364 & 0.0042 & 0.5174 & 0.0369 & 162.451 & 37.474 & 17.1 & 3100 & ODP1207 & Seki et al. 2066 \\
\hline 0.3339 & 0.0567 & 0.0511 & 0.0080 & 0.5180 & 0.0324 & 158.121 & 36.763 & 18.3 & 3345 & ODP1208 & Seki et al. 2067 \\
\hline 0.2990 & 0.0575 & 0.0540 & 0.0097 & 0.5381 & 0.0417 & 157.51 & 32.013 & 21.4 & 2907 & ODP1211 & Seki et al. 2068 \\
\hline 0.3020 & 0.0584 & 0.0523 & 0.0087 & 0.5408 & 0.0378 & 157.427 & 32.269 & 21.4 & 2682 & ODP1212 & Seki et al. 2069 \\
\hline
\end{tabular}


Eocene dataset for OPTiMAL

\begin{tabular}{|c|c|c|c|c|c|c|c|c|c|c|c|c|c|c|c|c|c|}
\hline SITE & AGE & $\begin{array}{c}1302 \\
\text { GDGTO }\end{array}$ & $\begin{array}{c}1300 \\
\text { GDGT1 }\end{array}$ & $\begin{array}{c}1298 \\
\text { GDGT2 } \\
\end{array}$ & $\begin{array}{c}1296 \\
\text { GDGT3 } \\
\end{array}$ & $\begin{array}{l}1292 \\
\text { CREN } \\
\end{array}$ & $\begin{array}{l}1292^{\prime} \\
\text { CREN }\end{array}$ & Fr. 1302 & Fr.1300 & Fr.1298 & Fr.1296 & Fr.1292 & Fr.1292' & $\begin{array}{c}\text { TEX } \\
\text { (published) }\end{array}$ & & $\begin{array}{c}\text { OPTIMAL } \\
\text { SST }\end{array}$ & $\begin{array}{l}\text { OPTIMAL } \\
\text { StDev }\end{array}$ \\
\hline 277 & 33.60 & 399262 & 72276 & 64028 & 12946 & 548757 & 39613 & 0.35 & 0.06 & 0.06 & 0.01 & 0.48 & 0.03 & 0.62 & 0.34 & 21.33 & 3.56 \\
\hline 277 & 33.62 & 455976 & 97219 & 88849 & 17494 & 665582 & 57692 & 0.33 & & 0.06 & 0.01 & 0.48 & 0.04 & 0.63 & 0.29 & 23.27 & \\
\hline 277 & 33.64 & 529802 & 125675 & 133551 & 27576 & 944662 & 81286 & 0.29 & 0.07 & 0.07 & 0.01 & 0.51 & 0.04 & 0.66 & 0.29 & 25.87 & 3.49 \\
\hline 277 & 33.64 & 95110 & 21042 & 22104 & 4411 & 158033 & 14683 & 0.30 & 0.07 & 0.07 & 0.01 & 0.50 & 0.05 & 0.66 & 0.29 & 25.51 & 3.54 \\
\hline 277 & 33.66 & 26399 & 5661 & 5666 & 1442 & 38412 & 3464 & 0.33 & 0.07 & 0.07 & 0.02 & 0.47 & 0.04 & 0.65 & 0.34 & 25.02 & 4.24 \\
\hline 277 & 33.69 & 290097 & 59779 & 76566 & 12952 & 395740 & 41152 & 0.33 & 0.07 & 0.09 & 0.01 & 0.45 & 0.05 & 0.69 & 0.50 & 28.01 & 4.15 \\
\hline 277 & 33.73 & 81897 & 15575 & 17157 & 3172 & 91606 & 9753 & 0.37 & 0.07 & 0.08 & 0.01 & 0.42 & 0.04 & 0.66 & 0.63 & 25.52 & 4.73 \\
\hline 277 & 33.83 & 556111 & 117435 & 120342 & 25453 & 828508 & 73815 & 0.32 & 0.07 & 0.07 & 0.01 & 0.48 & 0.04 & 0.65 & 0.40 & 25.50 & 3.68 \\
\hline 277 & 34.57 & 270004 & 59050 & 60130 & 10788 & 396784 & 37311 & 0.32 & 0.07 & 0.07 & 0.01 & 0.48 & 0.04 & 0.65 & 0.38 & 24.97 & 3.59 \\
\hline 277 & 34.69 & 34760 & 7243 & 7964 & 1584 & 46453 & 4557 & 0.34 & 0.07 & 0.08 & 0.02 & 0.45 & 0.04 & 0.66 & 0.32 & 26.62 & 4.03 \\
\hline 277 & $\begin{array}{l}34.75 \\
35.53\end{array}$ & $\begin{array}{l}82836 \\
24304\end{array}$ & 17912 & 20483 & $\begin{array}{l}3429 \\
\end{array}$ & 121929 & 12402 & 0.32 & 0.07 & 0.08 & 0.01 & 0.47 & 0.05 & 0.67 & 0.38 & 26.53 & 3.67 \\
\hline $\begin{array}{l}277 \\
925\end{array}$ & $\begin{array}{r}35.53 \\
285657\end{array}$ & $\begin{array}{r}243204 \\
371885\end{array}$ & $\begin{array}{l}51087 \\
37159\end{array}$ & $\begin{array}{r}66343 \\
43559\end{array}$ & $\begin{array}{l}11881 \\
8972\end{array}$ & $\begin{array}{l}322185 \\
217396\end{array}$ & $\begin{array}{l}40008 \\
30577\end{array}$ & $\begin{array}{l}0.33 \\
0.52\end{array}$ & 0.07 & 0.09 & 0.02 & 0.44 & 0.05 & 0.70 & 0.42 & $\begin{array}{l}28.14 \\
12.50\end{array}$ & 4.48 \\
\hline $\begin{array}{l}925 \\
925\end{array}$ & $\begin{array}{l}28.567 \\
29272\end{array}$ & $\begin{array}{c}371885 \\
489884\end{array}$ & $\begin{array}{l}37159 \\
276324\end{array}$ & $\begin{array}{r}43559 \\
3344266\end{array}$ & $\begin{array}{c}8972 \\
78956\end{array}$ & $\begin{array}{l}217396 \\
2164039\end{array}$ & $\begin{array}{l}30547 \\
283752\end{array}$ & $\begin{array}{l}0.52 \\
0.60\end{array}$ & 0.05 & 0.06 & 0.01 & 0.31 & 0.04 & 0.69 & $\begin{array}{l}1.17 \\
1.17\end{array}$ & $\begin{array}{l}12.50 \\
13.57\end{array}$ & $\begin{array}{l}7.00 \\
7.05\end{array}$ \\
\hline $\begin{array}{l}925 \\
925\end{array}$ & $\begin{array}{l}\begin{array}{l}29.272 \\
29.287\end{array} \\
2\end{array}$ & $\begin{array}{c}48398664 \\
222691\end{array}$ & $\begin{array}{c}2776324 \\
47899\end{array}$ & $\begin{array}{c}3742666 \\
59474\end{array}$ & $\begin{array}{l}78956 \\
13105\end{array}$ & $\begin{array}{l}21640399 \\
348407\end{array}$ & $\begin{array}{c}283752 \\
51332\end{array}$ & $\begin{array}{l}0.60 \\
0.30\end{array}$ & $\begin{array}{l}0.03 \\
0.06\end{array}$ & $\begin{array}{l}0.05 \\
0.08\end{array}$ & $\begin{array}{l}0.01 \\
0.02\end{array}$ & $\begin{array}{l}0.27 \\
0.47\end{array}$ & $\begin{array}{l}0.04 \\
0.07\end{array}$ & $\begin{array}{l}0.73 \\
0.72\end{array}$ & $\begin{array}{l}1.13 \\
0.61\end{array}$ & $\begin{array}{l}13.57 \\
27.69\end{array}$ & $\begin{array}{l}7.05 \\
4.85\end{array}$ \\
\hline 925 & 30.008 & 101302 & 27034 & 40719 & 8673 & 204521 & 33873 & $\begin{array}{l}0.24 \\
0.24\end{array}$ & $\begin{array}{l}0.06 \\
0.06\end{array}$ & $\begin{array}{l}0.08 \\
0.10\end{array}$ & $\begin{array}{l}0.02 \\
0.02\end{array}$ & $\begin{array}{l}0.47 \\
0.49\end{array}$ & 0.08 & $\begin{array}{l}0.12 \\
0.75\end{array}$ & $\begin{array}{l}0.71 \\
0.71\end{array}$ & $\begin{array}{l}27.69 \\
26.32\end{array}$ & $\begin{array}{l}4.85 \\
5.48\end{array}$ \\
\hline 925 & 30.560 & 235590 & 23178 & 28433 & 5256 & 127073 & 17324 & 0.54 & 0.05 & 0.07 & 0.01 & $\begin{array}{l}0.29 \\
0.29\end{array}$ & $\begin{array}{l}0.04 \\
0.04\end{array}$ & 0.69 & 1.22 & $\begin{array}{l}20.32 \\
14.93\end{array}$ & $\begin{array}{l}5.48 \\
7.13\end{array}$ \\
\hline 925 & & & & & 7295 & 181854 & & 0.27 & 0.07 & 0.09 & & 0.47 & 0.08 & 0.74 & 0.64 & 27.58 & 516 \\
\hline 925 & 30.779 & 461034 & 66172 & 99174 & 20818 & 552761 & 87526 & 0.36 & 0.05 & 0.08 & 0.02 & 0.43 & 0.07 & 0.76 & 0.96 & 25.76 & $\begin{array}{l}3.10 \\
6.34\end{array}$ \\
\hline 925 & 31.103 & 65752 & 15286 & 23368 & 4890 & 121458 & 18461 & 0.26 & 0.06 & 0.09 & 0.02 & 0.49 & 0.07 & 0.75 & 0.68 & 27.02 & $\begin{array}{l}0.35 \\
5.35\end{array}$ \\
\hline 925 & 31.724 & 139022 & 17935 & 22236 & 5010 & 137589 & 22126 & 0.40 & 0.05 & 0.06 & 0.01 & 0.40 & 0.06 & 0.73 & 1.08 & 22.15 & 6.54 \\
\hline 925 & 32.68 & 10931241 & 1465161 & 2096084 & 421840 & 14254692 & 1955273 & 0.35 & 0.05 & 0.07 & 0.01 & 0.46 & 0.06 & 0.75 & 0.79 & 25.89 & 5.50 \\
\hline 925 & 32.78 & 12687958 & 2572163 & 3977902 & 833161 & 26261962 & 3393341 & 0.26 & 0.05 & 0.08 & 0.02 & 0.53 & 0.07 & 0.76 & 0.67 & 28.50 & 4.81 \\
\hline 925 & 33.04 & 21001648 & 6201187 & 8310747 & 1751582 & 53691244 & 6885830 & 0.21 & 0.06 & 0.08 & 0.02 & 0.55 & 0.07 & 0.73 & 0.38 & 28.58 & 3.90 \\
\hline 925 & 33.27 & 162683 & 20988 & 29854 & 5632 & 275923 & 26313 & 0.31 & 0.04 & 0.06 & 0.01 & 0.53 & 0.05 & 0.75 & 0.62 & 22.80 & 4.72 \\
\hline 925 & 33.46 & 4724260 & 1234661 & 1813649 & 368949 & 12215482 & 1533550 & 0.22 & 0.06 & 0.08 & 0.02 & 0.56 & 0.07 & 0.75 & 0.51 & 28.94 & 4.11 \\
\hline 925 & 33.64 & 267958 & 73001 & 106371 & 22481 & 681789 & & 0.21 & 0.06 & 0.08 & 0.02 & 0.54 & 0.09 & 0.76 & 0.64 & 27.85 & \\
\hline 925 & 33.80 & 2592947 & 1152029 & 888343 & 177766 & 5507807 & 828296 & 0.23 & 0.10 & 0.08 & 0.02 & 0.49 & 0.07 & 0.62 & 1.00 & 18.38 & 6.42 \\
\hline 925 & 35.71 & 529883 & 102749 & 144066 & 27470 & 832557 & 122555 & 0.30 & 0.06 & 0.08 & 0.02 & 0.47 & 0.07 & 0.74 & 0.66 & 28.93 & 4.72 \\
\hline 925 & 35.70 & 872446 & 134704 & 183633 & 37166 & 1160097 & 168026 & 0.34 & 0.05 & 0.07 & 0.01 & 0.45 & 0.07 & 0.74 & 0.68 & 27.01 & 5.32 \\
\hline 925 & 35.75 & 2060238 & 357384 & 400825 & 81708 & 2741852 & 387629 & 0.34 & 0.06 & 0.07 & 0.01 & 0.45 & 0.06 & 0.71 & 0.67 & 26.25 & 4.68 \\
\hline 925 & 35.85 & 3783069 & 468151 & 454857 & 94610 & 2795988 & 439607 & 0.47 & 0.06 & 0.06 & 0.01 & 0.35 & 0.05 & 0.68 & 1.01 & 15.14 & 6.27 \\
\hline 925 & 36.00 & 935909 & 18247 & 26068 & 6027 & 165456 & 29825 & 0.79 & 0.02 & 0.02 & 0.01 & 0.14 & 0.03 & 0.77 & 0.85 & 9.99 & 6.71 \\
\hline 925 & 36.24 & 989460 & 262906 & 366016 & 76437 & 1893992 & 282011 & 0.26 & 0.07 & 0.09 & 0.02 & 0.49 & 07 & 0.73 & 0.57 & 27.55 & 4.87 \\
\hline 925 & 36.43 & 2601828 & 772026 & 1104442 & 243000 & 7674369 & 1045100 & 0.19 & 0.06 & 0.08 & 0.02 & 0.57 & 0.08 & 0.76 & 0.59 & 28.01 & 4.21 \\
\hline 925 & $\begin{array}{l}36.74 \\
37.01\end{array}$ & $\begin{array}{l}3114479 \\
253526\end{array}$ & $\begin{array}{l}1041854 \\
7567\end{array}$ & $\begin{array}{l}1186804 \\
13439\end{array}$ & $\begin{array}{l}288160 \\
30143\end{array}$ & $\begin{array}{l}8315579 \\
883883\end{array}$ & $\begin{array}{l}1233779 \\
15656\end{array}$ & 0.21 & 0.07 & 0.08 & 0.02 & 0.55 & 0.08 & 0.72 & 0.44 & $\begin{array}{l}27.34 \\
25.71\end{array}$ & $\begin{array}{l}4.39 \\
4.97\end{array}$ \\
\hline $\begin{array}{l}925 \\
925\end{array}$ & $\begin{array}{l}37.01 \\
37.25\end{array}$ & $\begin{array}{c}253526 \\
1110453\end{array}$ & $\begin{array}{c}75267 \\
369832\end{array}$ & $\begin{array}{l}134389 \\
44288\end{array}$ & $\begin{array}{l}30143 \\
99963\end{array}$ & $\begin{array}{c}883883 \\
286099\end{array}$ & $\begin{array}{l}156556 \\
444043\end{array}$ & $\begin{array}{l}0.17 \\
0.22\end{array}$ & 0.05 & 0.09 & 0.02 & $\begin{array}{l}0.58 \\
0.53\end{array}$ & 0.10 & 0.81 & $\begin{array}{l}0.54 \\
0.45\end{array}$ & $\begin{array}{l}25.71 \\
27.59\end{array}$ & $\begin{array}{l}4.92 \\
4.37\end{array}$ \\
\hline $\begin{array}{l}925 \\
925\end{array}$ & $\begin{array}{l}37.25 \\
37.51\end{array}$ & $\begin{array}{l}11160453 \\
11812031\end{array}$ & $\begin{array}{l}369832 \\
3448737\end{array}$ & $\begin{array}{l}442288 \\
5030909\end{array}$ & $\begin{array}{l}99963 \\
854215\end{array}$ & $\begin{array}{l}28809991 \\
33264954\end{array}$ & $\begin{array}{r}4440453 \\
4902532\end{array}$ & $\begin{array}{l}0.22 \\
0.20\end{array}$ & $\begin{array}{l}0.07 \\
0.06\end{array}$ & $\begin{array}{l}0.08 \\
0.08\end{array}$ & $\begin{array}{l}0.02 \\
0.01\end{array}$ & $\begin{array}{l}0.53 \\
0.56\end{array}$ & $\begin{array}{l}0.08 \\
0.08\end{array}$ & $\begin{array}{l}0.73 \\
0.76\end{array}$ & $\begin{array}{l}0.45 \\
0.45\end{array}$ & $\begin{array}{l}27.59 \\
28.96\end{array}$ & $\begin{array}{l}4.37 \\
4.12\end{array}$ \\
\hline $\begin{array}{l}925 \\
925\end{array}$ & $\begin{array}{l}38.51 \\
38.02\end{array}$ & $\begin{array}{c}11818031 \\
23210\end{array}$ & $\begin{array}{c}3488 / 37 \\
2442\end{array}$ & $\begin{array}{c}5030009 \\
5020\end{array}$ & $\begin{array}{c}854215 \\
1117\end{array}$ & $\begin{array}{c}33264954 \\
31718\end{array}$ & $\begin{array}{c}49022352 \\
6115\end{array}$ & $\begin{array}{l}0.20 \\
0.33\end{array}$ & $\begin{array}{l}0.06 \\
0.04\end{array}$ & $\begin{array}{l}0.08 \\
0.07\end{array}$ & $\begin{array}{l}0.01 \\
0.02\end{array}$ & $\begin{array}{l}0.56 \\
0.46\end{array}$ & $\begin{array}{l}0.08 \\
0.09\end{array}$ & $\begin{array}{l}0.76 \\
0.83\end{array}$ & $\begin{array}{l}0.45 \\
1.21\end{array}$ & $\begin{array}{l}28.96 \\
23.88\end{array}$ & $\begin{array}{l}4.12 \\
7.61\end{array}$ \\
\hline $\begin{array}{l}925 \\
925\end{array}$ & 38.27 & 2102060 & 451032 & 646792 & 144756 & 4601664 & 708418 & 0.24 & $\begin{array}{l}0.04 \\
0.05\end{array}$ & 0.07 & 0.02 & $\begin{array}{l}0.46 \\
0.53\end{array}$ & 0.08 & $\begin{array}{l}0.77 \\
0.77\end{array}$ & $\begin{array}{l}1.21 \\
0.77\end{array}$ & 2831 & $\begin{array}{l}7.61 \\
5.14\end{array}$ \\
\hline 作 & 38.54 & 707844 & 192124 & 310691 & 58687 & 1672801 & $\begin{array}{l}100410 \\
273167\end{array}$ & $\begin{array}{l}0.24 \\
0.22\end{array}$ & 0.06 & 0.10 & 0.02 & 0.52 & 0.08 & 0.77 & 0.59 & 27.8 & $\begin{array}{l}5.14 \\
4.77\end{array}$ \\
\hline 925 & 38.49 & 161284 & 51575 & 79878 & 19309 & 525104 & 92933 & 0.17 & 0.06 & 0.09 & 0.02 & 0.56 & 0.10 & 0.79 & 0.46 & 25.39 & 4.91 \\
\hline 925 & 38.33 & 189644 & 49185 & 69933 & 13000 & 331586 & 53212 & 0.27 & 0.07 & 0.10 & 0.02 & 0.47 & 0.08 & 0.73 & 0.65 & 28.05 & 4.91 \\
\hline 925 & 38.69 & 26977 & 3856 & 7053 & 1430 & 49692 & 10170 & 0.27 & 0.04 & 0.07 & 0.01 & 0.50 & 0.10 & 0.83 & 1.19 & 26.78 & 6.97 \\
\hline 511 & 31.836 & 7585985 & 995499 & 558441 & 100994 & 5516933 & 305485 & 0.50 & 0.07 & 0.04 & 0.01 & 0.37 & 0.02 & 0.49 & 0.15 & 12.61 & 3.51 \\
\hline 511 & 32.412 & 16804794 & 1598778 & 665883 & 136655 & 9806814 & 297738 & 0.57 & 0.05 & 0.02 & 0.00 & 0.33 & 0.01 & 0.41 & 0.26 & 6.90 & 3.49 \\
\hline 511 & 32.58 & 30597348 & 3238354 & 1446366 & 254525 & 20519746 & 747760 & 0.54 & 0.06 & 0.03 & 0.00 & 0.36 & 0.01 & 0.43 & 0.20 & 6.45 & 3.43 \\
\hline 511 & 32.76 & 27495930 & 3341567 & 1575671 & 303175 & 18968080 & 775932 & 0.52 & 0.06 & 0.03 & 0.01 & 0.36 & 0.01 & 0.44 & 0.31 & 9.27 & 3.48 \\
\hline 511 & 32.89 & 10398928 & 1091973 & 499850 & 95828 & 6616941 & 251331 & 0.55 & 0.06 & 0.03 & 0.01 & 0.35 & 0.01 & 0.44 & 0.21 & 7.94 & 3.48 \\
\hline 511 & 33.17 & 21264646 & 1631833 & 629846 & 147125 & 12274376 & 305591 & 0.59 & 0.05 & 0.02 & 0.00 & 0.34 & 0.01 & 0.40 & 0.15 & 5.93 & 3.47 \\
\hline 511 & 33.28 & 14619175 & 1257344 & 548795 & 125863 & 9649294 & 293331 & 0.55 & 0.05 & 0.02 & 0.00 & 0.36 & 0.01 & 0.43 & 0.24 & 8.03 & 3.43 \\
\hline 511 & $\begin{array}{r}33.45 \\
33.45\end{array}$ & $\begin{array}{l}18397468 \\
11184108\end{array}$ & $\begin{array}{l}2007194 \\
838291\end{array}$ & $\begin{array}{l}937665 \\
337519\end{array}$ & $\begin{array}{l}181289 \\
79050\end{array}$ & $\begin{array}{l}12311290 \\
5470280\end{array}$ & $\begin{array}{l}439843 \\
110447\end{array}$ & 0.54 & 0.06 & $\begin{array}{l}0.03 \\
0.02\end{array}$ & 0.01 & 0.36 & 0.01 & 0.44 & $\begin{array}{l}0.20 \\
0.41\end{array}$ & 8.76 & $\begin{array}{l}3.47 \\
3.60\end{array}$ \\
\hline $\begin{array}{l}511 \\
511\end{array}$ & $\begin{array}{l}33.48 \\
33.59\end{array}$ & $\begin{array}{l}11184109 \\
2624844\end{array}$ & $\begin{array}{l}838291 \\
2225182\end{array}$ & $\begin{array}{l}337519 \\
885846\end{array}$ & $\begin{array}{l}79050 \\
207003\end{array}$ & $\begin{array}{c}5470280 \\
14617906\end{array}$ & $\begin{array}{l}160447 \\
398521\end{array}$ & $\begin{array}{l}0.62 \\
0.59\end{array}$ & $\begin{array}{l}0.05 \\
0.05\end{array}$ & $\begin{array}{l}0.02 \\
0.02\end{array}$ & $\begin{array}{l}0.00 \\
0.00\end{array}$ & $\begin{array}{l}0.30 \\
0.33\end{array}$ & $\begin{array}{l}0.01 \\
0.01\end{array}$ & $\begin{array}{l}0.41 \\
0.40\end{array}$ & $\begin{array}{l}0.41 \\
0.27\end{array}$ & 6.22 & $\begin{array}{l}3.60 \\
3.53\end{array}$ \\
\hline $\begin{array}{l}511 \\
511\end{array}$ & $\begin{array}{l}33.59 \\
3.61\end{array}$ & $\begin{array}{l}262448444 \\
17122232\end{array}$ & $\begin{array}{l}2225182 \\
1642815\end{array}$ & $\begin{array}{l}8858466 \\
681837\end{array}$ & $\begin{array}{l}207003 \\
143143\end{array}$ & $\begin{array}{c}1461717006 \\
9388046\end{array}$ & $\begin{array}{l}398521 \\
299333\end{array}$ & $\begin{array}{l}0.59 \\
0.58\end{array}$ & $\begin{array}{l}0.05 \\
0.06\end{array}$ & $\begin{array}{l}0.02 \\
0.02\end{array}$ & $\begin{array}{l}0.00 \\
0.00\end{array}$ & $\begin{array}{l}0.33 \\
0.32\end{array}$ & $\begin{array}{l}0.01 \\
0.01\end{array}$ & $\begin{array}{l}0.40 \\
0.41\end{array}$ & $\begin{array}{l}0.27 \\
0.31\end{array}$ & $\begin{array}{l}7.19 \\
7.22\end{array}$ & $\begin{array}{l}3.53 \\
3.56\end{array}$ \\
\hline 511 & $\begin{array}{l}3.61 \\
34.04\end{array}$ & 3776361 & 425820 & $\begin{array}{l}688387 \\
194989\end{array}$ & $\begin{array}{l}1435143 \\
33837\end{array}$ & $\begin{array}{l}93580046 \\
2184045\end{array}$ & $\begin{array}{l}9293333 \\
91636\end{array}$ & $\begin{array}{l}0.58 \\
0.56\end{array}$ & 0.06 & $\begin{array}{l}0.02 \\
0.03\end{array}$ & 0.01 & 0.33 & 0.01 & $\begin{array}{l}0.41 \\
0.43\end{array}$ & $\begin{array}{l}0.31 \\
0.34\end{array}$ & 7.28 & $\begin{array}{l}3.56 \\
3.56\end{array}$ \\
\hline 511 & $\begin{array}{l}54.04 \\
34.19\end{array}$ & 125902696 & $\begin{array}{l}425080 \\
1454180\end{array}$ & 7688243 & 2071190 & $\begin{array}{l}216404040 \\
116072024\end{array}$ & 4565806 & 0.46 & 0.05 & 0.0 & 0.01 & 年 & 0.0 & $\begin{array}{l}0.45 \\
0.50\end{array}$ & $\begin{array}{l}0.34 \\
0.32\end{array}$ & 1057 & $\begin{array}{l}3.30 \\
3.52\end{array}$ \\
\hline 511 & 34.20 & 65363492 & 8723104 & 4936521 & 1286238 & 66754182 & 3011769 & 0.44 & 0.06 & 00 & 0.0 & 0. & 0 & 0.5 & 0 & 1293 & 3.53 \\
\hline 511 & 34.32 & 8042662 & 986513 & 510336 & $\begin{array}{l}100263 \\
1003\end{array}$ & 7089987 & 277396 & 0.47 & 0.06 & 0.03 & 0.01 & 0.42 & 0.02 & 0.47 & 0.13 & 10.71 & 3.43 \\
\hline 511 & 34.37 & 10063078 & 1319430 & 720036 & 154048 & 10124850 & 418549 & 0.44 & 0.06 & 0.03 & 0.01 & 0.44 & 0.02 & 0.49 & 0.20 & 12.93 & 3.46 \\
\hline 511 & 35.36 & 59190812 & 10303085 & 7122944 & 1284026 & 70778736 & 4441188 & 0.39 & 0.07 & 0.05 & 0.01 & 0.46 & 0.03 & 0.55 & 0.29 & 17.99 & 3.48 \\
\hline 929 & 34.86 & 131784336 & 33704876 & 43190608 & 8970174 & 218540928 & 38366452 & 0.28 & 0.07 & 0.09 & 0.02 & 0.46 & 0.08 & 0.73 & 0.68 & 27.27 & 5.27 \\
\hline 929 & 35.48 & 140806240 & 34898272 & 47702704 & 9664573 & 266739664 & 45774904 & 0.26 & 0.06 & 0.09 & 0.02 & 0.49 & 0.08 & 0.75 & 0.70 & 28.30 & 5.00 \\
\hline 929 & 36.07 & 6671968 & 464072 & 694019 & 122735 & 3015787 & 517003 & 0.58 & 0.04 & 0.06 & 0.01 & 0.26 & 0.05 & 0.74 & 1.25 & 14.93 & 7.60 \\
\hline 929 & 36.70 & 4599892 & 197736 & 251904 & 37154 & 1017590 & 167860 & 0.73 & 0.03 & 0.04 & 0.01 & 0.16 & 0.03 & 0.70 & 0.94 & 9.78 & 6.75 \\
\hline 929 & 37.31 & 90363 & 6991 & 8657 & 1500 & 49586 & 7273 & 0.55 & 0.04 & 0.05 & 0.01 & 0.30 & 0.04 & 0.71 & 1.04 & 14.01 & 6.45 \\
\hline 929 & 37.94 & 18545756 & 918926 & 1264245 & 221522 & 5721923 & 891261 & & 0.03 & 0.05 & 0.01 & 0.21 & 0.03 & 0.72 & 1.11 & 13.19 & 7.22 \\
\hline 929 & 43.52 & 2172128 & 167938 & 267906 & 38797 & 1090903 & 215376 & 0.55 & 0.04 & & 0.01 & 0.28 & 0.05 & 0.76 & 1.34 & 15.62 & 7.59 \\
\hline 929 & 48.28 & 138956 & 7825 & 11866 & 4000 & 60672 & 12525 & 0.59 & 0.03 & 0.05 & 0.02 & 0.26 & 0.05 & 0.78 & 1.40 & 9.71 & 8.26 \\
\hline
\end{tabular}


Eocene dataset for OPTiMAL

\begin{tabular}{|c|c|c|c|c|c|c|c|c|c|c|c|c|c|c|c|c|c|}
\hline SITE & AGE & $\begin{array}{c}1302 \\
\text { GDGTO }\end{array}$ & $\begin{array}{l}1300 \\
\text { GDGT1 }\end{array}$ & $\begin{array}{c}1298 \\
\text { GDGT2 }\end{array}$ & $\begin{array}{l}1296 \\
\text { GDGT3 }\end{array}$ & $\begin{array}{l}1292 \\
\text { CREN }\end{array}$ & $\begin{array}{l}1292^{\prime} \\
\text { CREN }^{\prime}\end{array}$ & Fr. 1302 & Fr. 1300 & Fr.1298 & Fr.1296 & Fr.1292 & Fr.1292' & $\begin{array}{c}\text { TEX } \\
\text { (published) }\end{array}$ & & $\begin{array}{c}\text { OPTTMAL } \\
\text { SST }\end{array}$ & $\begin{array}{c}\text { OPTTMAL } \\
\text { StDev }\end{array}$ \\
\hline 929 & 49.84 & 1992415 & 13190 & 40102 & 22270 & 85480 & 14475 & 0.92 & 0.01 & 0.02 & 0.01 & 0.04 & 0.01 & 0.85 & 2.48 & $\begin{array}{l}5.10 \\
15.10\end{array}$ & 8.89 \\
\hline 929 & 52.97 & 24658 & 2120 & 4993 & 1970 & 13689 & 1124 & 0.51 & 0.04 & & 0.04 & 0.28 & 0.02 & 0.79 & 3.73 & 15.55 & 8.91 \\
\hline 929 & 54.12 & 182070 & 32153 & 58059 & 11531 & 351952 & 49574 & 0.27 & 0.05 & 0.08 & 0.02 & 0.51 & 0.07 & 0.79 & 0.85 & 27.74 & 5.61 \\
\hline 929 & 36.64 & 4780792 & 172648 & 149235 & 26302 & 1008010 & 173110 & 0.76 & 0.03 & 0.02 & 0.00 & 0.16 & 0.03 & 0.67 & 0.56 & 7.54 & 5.53 \\
\hline 929 & 37.83 & 6313019 & 1624554 & 2668454 & 552932 & 14169613 & 2689438 & 0.23 & 0.06 & 0.10 & 0.02 & 0.51 & 0.10 & 0.78 & 0.66 & 25.69 & 5.58 \\
\hline Mid Waipara & 42.00 & 6070000 & 1560000 & 1140000 & 417000 & 12000000 & 477000 & 0.28 & 0.07 & 0.05 & 0.02 & 0.55 & 0.02 & 0.57 & 0.47 & 20.86 & 4.03 \\
\hline Mid Waipara & 45.78 & 1050000 & 390000 & 500000 & 205000 & 3800000 & 359000 & 0.17 & 0.06 & 0.08 & 0.03 & 0.60 & 0.06 & 0.73 & 1.17 & 22.61 & 7.52 \\
\hline Mid Waipara & 45.85 & 683000 & 268000 & 341000 & 140000 & 2450000 & 213000 & 0.17 & 0.07 & 0.08 & 0.03 & 0.60 & 0.05 & 0.72 & 0.82 & 23.00 & 6.84 \\
\hline Mid Waipara & $\begin{array}{l}46.15 \\
4652\end{array}$ & 3910000 & $\begin{array}{r}1270000 \\
331900\end{array}$ & $\begin{array}{l}1470000 \\
37700\end{array}$ & $\begin{array}{l}603000 \\
15000\end{array}$ & 10400000 & 812000 & 0.21 & 0.07 & 0.08 & 0.03 & 0.56 & 0.04 & 0.69 & 1.11 & 21.55 & 7.64 \\
\hline $\begin{array}{l}\text { Mid Waipara } \\
\text { Mid Waijara }\end{array}$ & $\begin{array}{l}46.52 \\
46.64\end{array}$ & $\begin{array}{l}36700000 \\
540000\end{array}$ & $\begin{array}{l}3190000 \\
1930000\end{array}$ & $\begin{array}{c}327000 \\
2300000\end{array}$ & $\begin{array}{r}145000 \\
1070000\end{array}$ & $\begin{array}{l}19900000 \\
15100000\end{array}$ & $\begin{array}{c}131000 \\
1200000\end{array}$ & $\begin{array}{l}0.56 \\
0.20\end{array}$ & $\begin{array}{l}0.05 \\
0.07\end{array}$ & $\begin{array}{l}0.05 \\
0.09\end{array}$ & $\begin{array}{l}0.02 \\
0.04\end{array}$ & $\begin{array}{l}0.30 \\
0.56\end{array}$ & $\begin{array}{l}0.02 \\
0.04\end{array}$ & 0.65 & $\begin{array}{l}0.98 \\
0.55\end{array}$ & $\begin{array}{l}10.15 \\
24.74\end{array}$ & $\begin{array}{l}7.28 \\
5.84\end{array}$ \\
\hline $\begin{array}{l}\text { Mid Walarara } \\
\text { Mid Waipara }\end{array}$ & 46.88 & 3920000 & 1340000 & 1470000 & $\begin{array}{l}10700000 \\
573000\end{array}$ & $\begin{array}{l}15100000 \\
11500000\end{array}$ & $\begin{array}{l}12000000 \\
846000\end{array}$ & $\begin{array}{l}0.20 \\
0.20\end{array}$ & $\begin{array}{l}0.07 \\
0.07\end{array}$ & $\begin{array}{l}0.09 \\
0.07\end{array}$ & $\begin{array}{l}0.04 \\
0.03\end{array}$ & $\begin{array}{l}0.56 \\
0.59\end{array}$ & $\begin{array}{l}0.04 \\
0.04\end{array}$ & $\begin{array}{l}0.70 \\
0.68\end{array}$ & $\begin{array}{l}0.55 \\
1.22\end{array}$ & $\begin{array}{l}24.74 \\
2160\end{array}$ & $\begin{array}{l}5.84 \\
7.57\end{array}$ \\
\hline Mid Waipara & 47.16 & 713000 & 266000 & 307000 & 131000 & 2390000 & 195000 & 0.18 & 0.07 & 0.08 & $\begin{array}{l}0.03 \\
0.03\end{array}$ & $\begin{array}{l}0.59 \\
0.60\end{array}$ & $\begin{array}{l}0.04 \\
0.05\end{array}$ & $\begin{array}{l}0.68 \\
0.70\end{array}$ & $\begin{array}{l}1.222 \\
1.07\end{array}$ & $\begin{array}{l}\begin{array}{l}21.60 \\
22.30\end{array} \\
2.0\end{array}$ & $\begin{array}{l}7.57 \\
7.41\end{array}$ \\
\hline Mid Waipara & 47.53 & 13300000 & 4800000 & 5460000 & 2310000 & 39700000 & 3200000 & 0.19 & 0.07 & 0.08 & 0.03 & 0.58 & 0.05 & 0.70 & 0.89 & 22.35 & $\begin{array}{l}7.41 \\
7.07\end{array}$ \\
\hline Mid Waipara & 47.89 & 13500000 & 6330000 & 7050000 & 2880000 & 49500000 & 4650000 & 0.16 & 0.08 & 0.08 & 0.03 & 0.59 & 0.06 & 0.70 & 0.89 & 22.18 & 7.13 \\
\hline & 48.26 & 3110000 & 1260000 & 1750000 & 740000 & 12800000 & 1530000 & 0.1 & 0.06 & 0.08 & 0.03 & 0.60 & 0.07 & 0.76 & 0.98 & 22.56 & 741 \\
\hline Mid Waipara & 48.63 & 368000 & 173000 & 244000 & 109000 & 1810000 & 225000 & 0.13 & 0.06 & 0.08 & 0.04 & 0.62 & 0.08 & 0.77 & 1.00 & 22.23 & $\begin{array}{l}.1 .41 \\
7.56\end{array}$ \\
\hline Mid Waipara & 49.00 & 5660000 & 2170000 & 2850000 & 1230000 & 19700000 & 1990000 & 0.17 & 0.06 & 0.08 & 0.04 & 0.59 & 0.06 & 0.74 & 0.53 & 23.99 & 5.90 \\
\hline Mid Waipara & 49.41 & 2520000 & 1080000 & 1510000 & 514000 & 10800000 & 1300000 & 0.14 & 0.06 & 0.09 & 0.03 & 0.61 & 0.07 & 0.75 & 0.72 & 24.41 & 6.15 \\
\hline Mid Waipara & $\begin{array}{l}49.81 \\
49.86\end{array}$ & 1380000 & 564000 & 838000 & 347000 & 6230000 & 805000 & 0.14 & 0.06 & 0.08 & 0.03 & 0.61 & 0.08 & 0.78 & 1.24 & 22.08 & 7.90 \\
\hline Mid Waipara & 50.31 & 176000 & 76000 & 111000 & 44900 & 849000 & 117000 & 0.13 & 0.06 & 0.08 & 0.03 & 0.62 & 0.09 & 0.78 & 1.51 & 22.31 & 7.92 \\
\hline Mid Waipara & 50.77 & 4290000 & 1750000 & 2400000 & 918000 & 17700000 & 1900000 & 0.15 & 0.06 & 0.08 & 0.03 & 0.61 & 0.07 & 0.75 & 1.30 & 22.88 & 7.55 \\
\hline Mid Waipara & 51.22 & 1750000 & 883000 & 1440000 & 645000 & 11800000 & 1540000 & 0.10 & 0.05 & 0.08 & 0.04 & 0.65 & 0.09 & 0.80 & 1.47 & 20.28 & 8.44 \\
\hline Mid Waipara & 51.52 & 122000 & 49100 & 75700 & 33100 & 648000 & 95400 & 0.12 & 0.05 & 0.07 & 0.03 & 0.63 & 0.09 & 0.81 & 1.46 & 22.03 & 7.99 \\
\hline Mid Waipara & 51.67 & 2320000 & 1080000 & 1780000 & 716000 & 13200000 & 1840000 & 0.11 & 0.05 & 0.09 & 0.03 & 0.63 & 0.09 & 0.80 & 1.50 & 21.20 & 8.27 \\
\hline $\begin{array}{l}\text { Mid Waipara } \\
\text { Mid Waiapara }\end{array}$ & $\begin{array}{l}53.18 \\
54.83\end{array}$ & 0.108 & $\begin{array}{l}0.048 \\
0.065\end{array}$ & $\begin{array}{l}0.084 \\
0.072\end{array}$ & $\begin{array}{l}0.030 \\
0.021\end{array}$ & $\begin{array}{l}0.635 \\
0.563\end{array}$ & $\begin{array}{l}0.096 \\
\end{array}$ & 0.11 & 0.05 & 0.08 & 0.03 & $\begin{array}{l}0.63 \\
0.56\end{array}$ & 0.10 & 0.82 & 0.94 & 24.59 & 6.68 \\
\hline $\begin{array}{l}\text { Mid Waipara } \\
\text { Mid Waipara }\end{array}$ & $\begin{array}{l}54.83 \\
5.23\end{array}$ & $\begin{array}{l}0.231 \\
0.223\end{array}$ & $\begin{array}{l}0.065 \\
0.065\end{array}$ & $\begin{array}{l}0.072 \\
0.071\end{array}$ & $\begin{array}{l}0.021 \\
0.024\end{array}$ & $\begin{array}{l}0.563 \\
0.573\end{array}$ & $\begin{array}{l}0.049 \\
0.045\end{array}$ & $\begin{array}{l}0.23 \\
0.22\end{array}$ & $\begin{array}{l}0.07 \\
0.06\end{array}$ & $\begin{array}{l}0.07 \\
0.07\end{array}$ & $\begin{array}{l}0.02 \\
0.02\end{array}$ & $\begin{array}{l}0.56 \\
0.57\end{array}$ & $\begin{array}{l}0.05 \\
0.04\end{array}$ & $\begin{array}{l}0.68 \\
0.68\end{array}$ & $\begin{array}{l}0.35 \\
0.82\end{array}$ & $\begin{array}{l}26.95 \\
2460\end{array}$ & $\begin{array}{l}4.20 \\
5.54\end{array}$ \\
\hline Hampden Beach & 41.20 & 7030000 & 1970000 & 1620000 & 814000 & 14500000 & $\begin{array}{l}.0 .045 \\
571000\end{array}$ & 0.27 & 0.07 & 0.06 & $\begin{array}{l}0.02 \\
0.03\end{array}$ & $\begin{array}{l}0.57 \\
0.55\end{array}$ & $\begin{array}{l}0.04 \\
0.02\end{array}$ & $\begin{array}{l}0.68 \\
0.60\end{array}$ & $\begin{array}{l}0.82 \\
1.77\end{array}$ & $\begin{array}{l}24.60 \\
18.08\end{array}$ & $\begin{array}{l}5.54 \\
8.58\end{array}$ \\
\hline Hampden Beach & 41.21 & 6860000 & 1910000 & 1580000 & 747000 & 14300000 & 577000 & 0.26 & 0.07 & 0.06 & 0.03 & 0.55 & 0.02 & 0.60 & 1.57 & 18.06 & 8.32 \\
\hline Hampden Beach & 41.22 & 2870000 & 775000 & 645000 & 305000 & 6190000 & 280000 & 0.26 & 0.07 & 0.06 & 0.03 & 0.56 & 0.03 & 0.61 & 1.32 & 18.54 & 7.87 \\
\hline Hampden Beach & 41.23 & 3100000 & 903000 & 762000 & 362000 & 6940000 & 290000 & 0.25 & 0.07 & 0.06 & 0.03 & 0.56 & 0.02 & 0.61 & 1.70 & 18.48 & 8.38 \\
\hline $\begin{array}{l}\text { Hampden Beach } \\
\text { Hampden Beaca }\end{array}$ & $\begin{array}{l}41.24 \\
41.25\end{array}$ & $\begin{array}{l}2980000 \\
2270000\end{array}$ & $\begin{array}{l}887000 \\
645000\end{array}$ & $\begin{array}{l}743000 \\
55500\end{array}$ & 379000 & 6840000 & 303000 & 0.25 & 0.07 & 0.06 & 0.03 & 0.56 & 0.02 & 0.62 & 1.56 & 18.92 & 8.44 \\
\hline $\begin{array}{l}\text { Hampden Beach } \\
\text { Hampden Beach }\end{array}$ & $\begin{array}{l}41.25 \\
41.28\end{array}$ & $\begin{array}{l}227700000 \\
4020000\end{array}$ & $\begin{array}{l}6450000 \\
1180000\end{array}$ & $\begin{array}{l}5520000 \\
1040000\end{array}$ & $\begin{array}{l}263000 \\
490000\end{array}$ & $\begin{array}{l}5010000 \\
9420000\end{array}$ & $\begin{array}{l}210000 \\
415000\end{array}$ & $\begin{array}{l}0.25 \\
0.24\end{array}$ & $\begin{array}{l}0.07 \\
0.07\end{array}$ & $\begin{array}{l}0.06 \\
0.06\end{array}$ & $\begin{array}{l}0.03 \\
0.03\end{array}$ & $\begin{array}{l}0.56 \\
0.57\end{array}$ & $\begin{array}{l}0.02 \\
0.03\end{array}$ & 0.61 & 1.71 & $\begin{array}{l}18.43 \\
18.88\end{array}$ & $\begin{array}{l}8.39 \\
88.35\end{array}$ \\
\hline Hampden Beach & 41.30 & 1030000 & 303000 & 263000 & 132000 & 2550000 & $\begin{array}{l}415000 \\
117000\end{array}$ & $\begin{array}{l}0.24 \\
0.23\end{array}$ & $\begin{array}{l}0.07 \\
0.07\end{array}$ & $\begin{array}{l}0.06 \\
0.06\end{array}$ & $\begin{array}{l}0.03 \\
0.03\end{array}$ & $\begin{array}{l}0.57 \\
0.58\end{array}$ & $\begin{array}{l}0.03 \\
0.03\end{array}$ & $\begin{array}{l}0.62 \\
0.63\end{array}$ & $\begin{array}{l}1.72 \\
1.74\end{array}$ & $\begin{array}{l}18.88 \\
19.13\end{array}$ & $\begin{array}{l}8.35 \\
8.35\end{array}$ \\
\hline Hampden Beach & 41.31 & 4940000 & 1400000 & 1170000 & 602000 & 10400000 & 419000 & 0.26 & 0.07 & 0.06 & 0.03 & 0.55 & 0.02 & 0.61 & 1.55 & 18.57 & $\begin{array}{l}8.35 \\
8.50\end{array}$ \\
\hline Hampden Beach & 41.32 & 2190000 & 579000 & 457000 & 231000 & 4530000 & 200000 & 0.27 & 0.07 & 0.06 & 0.03 & 0.55 & 0.02 & 0.61 & 1.42 & 17.94 & $\begin{array}{l}8.50 \\
8.15\end{array}$ \\
\hline Hampden Beach & 41.33 & 8950000 & 2560000 & 2150000 & 1010000 & 19200000 & 817000 & 0.26 & 0.07 & 0.06 & 0.03 & 0.55 & 0.02 & 0.61 & 1.66 & 18.28 & 8.38 \\
\hline Hampden Beach & 41.35 & 1050000 & 289000 & 258000 & 130000 & 00 & 105000 & 0.25 & 0.07 & 0.06 & 0.03 & 0.56 & 0.03 & 0.63 & 1.58 & 18.87 & $\begin{array}{l}8.30 \\
8.46\end{array}$ \\
\hline Hampden Beach & 41.36 & 7060000 & 2050000 & 1750000 & 955000 & 16000000 & 708000 & 0.25 & 0.07 & 0.06 & 0.03 & 0.56 & 0.02 & 0.62 & 1.18 & 19.97 & 7.98 \\
\hline Hampden Beach & 41.37 & 5530000 & 1490000 & 1320000 & 700000 & 11300000 & 487000 & 0.27 & 0.07 & 0.06 & 0.03 & 0.54 & 0.02 & 0.63 & 1.27 & 19.40 & 8.18 \\
\hline Hampden Beach & 41.38 & 7329000 & 2030000 & 1710000 & 897000 & 14100000 & 676000 & 0.27 & 0.08 & 0.06 & 0.03 & 0.53 & 0.03 & 0.62 & 1.37 & 18.90 & 8.35 \\
\hline Hampden Beach & 45.67 & 4060000 & 1260000 & 1080000 & 482000 & 9460000 & 438000 & 0.24 & 0.08 & 0.06 & 0.03 & 0.56 & 0.03 & 0.61 & $\begin{array}{l}1.67 \\
1.60\end{array}$ & $\begin{array}{l}10.96 \\
18.96\end{array}$ & 8.23 \\
\hline Hampden Beach & 45.84 & 3850000 & 1280000 & 1230000 & 658000 & 9650000 & 496000 & 0.22 & 0.07 & 0.07 & 0.04 & 0.56 & 0.03 & 0.65 & 0.55 & 23.60 & 5.99 \\
\hline Hampden Beach & 46.22 & 1283867 & 322491 & 535613 & 421640 & 6215649 & 382291 & 0.14 & 0.04 & 0.06 & 0.05 & 0.68 & 0.04 & 0.81 & 0.76 & 23.64 & 6.72 \\
\hline Hampden Beach & 46.40 & 1175196 & 414128 & 450987 & 244098 & 4044745 & 310179 & 0.18 & 0.06 & 0.07 & 0.04 & 0.61 & 0.05 & 0.71 & 0.38 & 24.50 & 5.35 \\
\hline Hampden Beach & 46.59 & 1288854 & 445187 & 479881 & 196107 & 4703904 & 334938 & 0.17 & 0.06 & 0.06 & 0.03 & 0.63 & 0.04 & 0.69 & 0.78 & 23.04 & 6.12 \\
\hline Hampden Beach & 46.78 & 1191714 & 407108 & 519632 & $\begin{array}{l}184301 \\
209060\end{array}$ & 4562637 & 416880 & 0.16 & 0.06 & 0.07 & 0.03 & 0.63 & 0.06 & 0.73 & 0.53 & 24.67 & 5.11 \\
\hline $\begin{array}{l}\text { Hampden Beach } \\
\text { Hamoden Beaca }\end{array}$ & $\begin{array}{l}46.96 \\
47.15\end{array}$ & 1125696 & $\begin{array}{l}463469 \\
686173\end{array}$ & $\begin{array}{l}468625 \\
701210\end{array}$ & $\begin{array}{l}295960 \\
54664\end{array}$ & 5000014 & $\begin{array}{l}471417 \\
664507\end{array}$ & 0.14 & 0.06 & 0.06 & 0.04 & 0.64 & 0.06 & 0.73 & 0.77 & 23.58 & 6.75 \\
\hline $\begin{array}{l}\text { Hampden Beach } \\
\text { Hampden Beach }\end{array}$ & $\begin{array}{l}47.15 \\
47.53\end{array}$ & $\begin{array}{l}1711414 \\
1352045\end{array}$ & $\begin{array}{l}656113 \\
510448\end{array}$ & $\begin{array}{l}701210 \\
803195\end{array}$ & $\begin{array}{l}546647 \\
513114\end{array}$ & $\begin{array}{l}9946854 \\
7447245\end{array}$ & $\begin{array}{l}644597 \\
473567\end{array}$ & $\begin{array}{l}0.12 \\
0.12\end{array}$ & $\begin{array}{l}0.05 \\
0.05\end{array}$ & $\begin{array}{l}0.05 \\
0.07\end{array}$ & $\begin{array}{l}0.04 \\
0.05\end{array}$ & $\begin{array}{l}0.70 \\
0.67\end{array}$ & 0.05 & 0.74 & $\begin{array}{l}1.17 \\
0.63\end{array}$ & $\begin{array}{l}22.03 \\
2586\end{array}$ & $\begin{array}{l}7.75 \\
55.83\end{array}$ \\
\hline Hampden Beach & 47.71 & $\begin{array}{l}1352045 \\
1392619\end{array}$ & 510526 & 615196 & $\begin{array}{l}112472 \\
41242\end{array}$ & 6518477 & 575394 & $\begin{array}{l}0.12 \\
0.14\end{array}$ & 0.05 & $\begin{array}{l}0.07 \\
0.06\end{array}$ & $\begin{array}{l}0.05 \\
0.04\end{array}$ & $\begin{array}{l}0.67 \\
0.65\end{array}$ & $\begin{array}{l}0.04 \\
0.06\end{array}$ & $\begin{array}{l}0.78 \\
0.76\end{array}$ & $\begin{array}{l}0.063 \\
0.82\end{array}$ & $\begin{array}{l}25.86 \\
24.47\end{array}$ & $\begin{array}{l}5.83 \\
6.56\end{array}$ \\
\hline Hampden Beach & 47.90 & 1407503 & 482860 & 580741 & 367693 & 5776392 & 490957 & 0.15 & 0.05 & 0.06 & 0.04 & 0.63 & 0.05 & 0.75 & 0.91 & 25.05 & 6.27 \\
\hline Hampden Beach & 48.09 & 1424701 & 434910 & 507929 & 275620 & 5478357 & 470181 & 0.17 & 0.05 & 0.06 & 0.03 & 0.64 & 0.05 & 0.74 & 1.42 & 21.49 & 7.98 \\
\hline Hampden Beach & 48.27 & 737271 & 297579 & 482906 & 234064 & 542 & 64 & 0.09 & 0.04 & 0.06 & 0.03 & 0.69 & 0.08 & 0.82 & 1.18 & 22.09 & 7.56 \\
\hline Hampden Beach & 48.46 & 1084672 & $\begin{array}{l}374330 \\
54203\end{array}$ & 416591 & 387788 & 4536460 & 413287 & 0.15 & 0.05 & 0.06 & 0.05 & 0.63 & 0.06 & 0.76 & 0.95 & 22.94 & 7.39 \\
\hline $\begin{array}{l}\text { Hampden Beach } \\
\text { Hamoden Beach }\end{array}$ & $\begin{array}{l}48.83 \\
49.02\end{array}$ & $\begin{array}{l}1345295 \\
1629896\end{array}$ & $\begin{array}{l}453393 \\
66246\end{array}$ & $\begin{array}{l}541320 \\
885824\end{array}$ & $\begin{array}{l}416256 \\
524143\end{array}$ & $\begin{array}{l}6471650 \\
578223\end{array}$ & 600933 & 0.14 & 0.05 & 0.06 & 0.04 & 0.66 & 0.06 & 0.77 & 0.76 & $\begin{array}{l}23.66 \\
23.36\end{array}$ & 6.71 \\
\hline $\begin{array}{l}\text { Hampden Beach } \\
\text { Hampden Beach }\end{array}$ & $\begin{array}{l}49.02 \\
49.06\end{array}$ & $\begin{array}{l}16298966 \\
922254\end{array}$ & $\begin{array}{l}662446 \\
428403\end{array}$ & $\begin{array}{l}858284 \\
606666\end{array}$ & $\begin{array}{l}524143 \\
376293\end{array}$ & $\begin{array}{l}5748223 \\
4912090\end{array}$ & $\begin{array}{l}608831 \\
387609\end{array}$ & $\begin{array}{l}0.16 \\
0.12\end{array}$ & $\begin{array}{l}0.07 \\
0.06\end{array}$ & $\begin{array}{l}0.09 \\
0.08\end{array}$ & 0.05 & $\begin{array}{l}0.57 \\
0.64\end{array}$ & 0.06 & 0.75 & 0.90 & $\begin{array}{l}23.36 \\
26.68\end{array}$ & $\begin{array}{l}7.07 \\
5560\end{array}$ \\
\hline $\begin{array}{l}\text { Hampoden Beacn } \\
\text { Hampden Beach }\end{array}$ & 49.11 & 844473 & 358893 & $\begin{array}{l}0.00606 \\
471816\end{array}$ & $\begin{array}{l}302767 \\
30276\end{array}$ & $\begin{array}{l}499209032 \\
429602\end{array}$ & 352161 & $\begin{array}{l}0.12 \\
0.13\end{array}$ & $\begin{array}{l}0.06 \\
0.05\end{array}$ & $\begin{array}{l}0.08 \\
0.07\end{array}$ & $\begin{array}{l}0.05 \\
0.05\end{array}$ & $\begin{array}{l}0.64 \\
0.65\end{array}$ & $\begin{array}{l}0.05 \\
0.05\end{array}$ & $\begin{array}{l}0.76 \\
0.76\end{array}$ & $\begin{array}{l}0.54 \\
0.61\end{array}$ & $\begin{array}{l}26.68 \\
25.89\end{array}$ & $\begin{array}{l}5.60 \\
5.74\end{array}$ \\
\hline Hampden Beach & 49.14 & 2682180 & 1003331 & 1248996 & 717576 & 9048 & 922 & 0. & 0.06 & 0.08 & 0.05 & 0.58 & 0.06 & 0.74 & 0.84 & 24.77 & 6.49 \\
\hline Hampden Beach & 49.18 & 905 & 370855 & 379953 & 290 & 4444584 & 340 & 0.13 & 0.06 & 0.06 & 0.04 & 0.66 & 0.05 & 0.73 & 0.59 & 25.05 & 6.07 \\
\hline Hampden Beach & 49.38 & 2355000 & 996534 & 1491489 & 852868 & 9524824 & 1058650 & 0 & 0.06 & 0.09 & 0.05 & 0.59 & 0.07 & 0.77 & 0.92 & 23.85 & 6.93 \\
\hline Hampden Beach & 52.12 & 525874 & 231161 & 304808 & 183187 & 4790448 & 606200 & 0.08 & 0.03 & 0.05 & 0.03 & 0.72 & 0.09 & 0.83 & 1.24 & 20.82 & 7.78 \\
\hline Hampden Beach & 52.33 & $\begin{array}{l}632911 \\
511233\end{array}$ & $\begin{array}{l}279471 \\
165217\end{array}$ & 391272 & 244204 & 2883577 & 284843 & 0.13 & 0.06 & 0.08 & 0.05 & 0.61 & 0.06 & 0.77 & 0.78 & 25.23 & 6.27 \\
\hline $\begin{array}{l}\text { Hampden Beach } \\
\text { Hampden Beach }\end{array}$ & $\begin{array}{l}5.73 \\
53.34\end{array}$ & $\begin{array}{l}510233 \\
287453\end{array}$ & $\begin{array}{l}6165217 \\
124280\end{array}$ & $\begin{array}{l}3322274 \\
18597\end{array}$ & $\begin{array}{l}2662660 \\
105171\end{array}$ & $\begin{array}{l}3719895 \\
1266967\end{array}$ & $\begin{array}{l}337671 \\
138848\end{array}$ & $\begin{array}{l}0.10 \\
0.14\end{array}$ & $\begin{array}{l}0.03 \\
0.06\end{array}$ & $\begin{array}{l}0.06 \\
0.09\end{array}$ & $\begin{array}{l}0.05 \\
0.05\end{array}$ & $\begin{array}{l}0.70 \\
0.60\end{array}$ & $\begin{array}{l}0.06 \\
0.07\end{array}$ & $\begin{array}{l}0.85 \\
0.78\end{array}$ & $\begin{array}{l}1.35 \\
0.68\end{array}$ & $\begin{array}{l}21.93 \\
25.00\end{array}$ & $\begin{array}{l}8.10 \\
6.38\end{array}$ \\
\hline Hampden Beach & 53.45 & 615953 & 244051 & 360409 & 209314 & 3308842 & 406206 & 0.12 & 0.05 & 0.07 & 0.04 & 0.64 & 0.08 & 0.80 & 1.14 & 22.19 & 7.71 \\
\hline Hampden Beach & 53.72 & 550141 & 193912 & 461075 & 358192 & 3933154 & 359436 & 0.09 & 0.03 & 0.08 & 0.06 & 0.67 & 0.06 & 0.86 & 1.90 & 16.95 & 8.80 \\
\hline
\end{tabular}


Eocene dataset for OPTiMAL

\begin{tabular}{|c|c|c|c|c|c|c|c|c|c|c|c|c|c|c|c|c|c|}
\hline SITE & AGE & $\begin{array}{c}1302 \\
\text { GDGTO } \\
\end{array}$ & $\begin{array}{c}1300 \\
\text { GDGT1 }\end{array}$ & $\begin{array}{c}1298 \\
\text { GDGT2 } \\
\end{array}$ & $\begin{array}{c}1296 \\
\text { GDGT3 }\end{array}$ & $\begin{array}{l}1292 \\
\text { CREN }\end{array}$ & $\begin{array}{l}1292^{\prime} \\
\text { CREN' } \\
\end{array}$ & Fr. 1302 & Fr. 1300 & Fr.1298 & Fr.1296 & Fr. 1292 & Fr.1292' & $\begin{array}{c}\text { TEx } \\
\text { (published) }\end{array}$ & D_nearest & $\begin{array}{c}\text { OPTIMAL } \\
\text { SST } \\
\end{array}$ & $\begin{array}{c}\text { OPTiMAL } \\
\text { StDev }\end{array}$ \\
\hline Hampden Beach & 54.74 & 386204 & 131034 & & 92879 & 1421454 & 149933 & 0.17 & 0.06 & 0.06 & 0.04 & 0.61 & 0.06 & 0.75 & 0.85 & 24.57 & \\
\hline 1172 & 37.01 & 16700000 & 2820000 & 1990000 & 686000 & 19500000 & 851000 & 0.39 & 0.07 & 0.05 & 0.02 & 0.46 & 0.02 & 0.56 & 0.32 & 18.24 & 4.32 \\
\hline 1172 & 38.21 & 10500000 & 1770000 & 1300000 & 454000 & 12600000 & 650000 & 0.38 & 0.06 & 0.05 & 0.02 & 0.46 & 0.02 & 0.58 & 0.44 & 19.38 & 4.46 \\
\hline 1172 & 38.54 & 3720000 & 695000 & 492000 & 164000 & 4430000 & 262000 & 0.38 & 0.07 & 0.05 & 0.02 & 0.45 & 0.03 & 0.57 & 0.49 & 19.98 & 4.58 \\
\hline 1172 & 38.73 & 8360000 & 1620000 & 1150000 & 385000 & 9990000 & 553000 & 0.38 & 0.07 & 0.05 & 0.02 & 0.45 & 0.03 & 0.56 & 0.62 & 20.22 & 4.80 \\
\hline 1172 & 38.97 & 11100000 & 2170000 & 1670000 & 657000 & 13800000 & 807000 & 0.37 & 0.07 & 0.06 & 0.02 & 0.46 & 0.03 & 0.59 & 0.94 & 20.48 & 5.88 \\
\hline 1172 & 39.20 & $\begin{array}{l}14200000 \\
\end{array}$ & 3520000 & 2680000 & 923000 & 17400000 & 1470000 & 0.35 & 0.09 & 0.07 & 0.02 & 0.43 & 0.04 & 0.59 & 1.36 & 20.28 & 7.14 \\
\hline $\begin{array}{l}11172 \\
1172\end{array}$ & $\begin{array}{l}39.48 \\
39.78\end{array}$ & $\begin{array}{l}90500000 \\
9750000\end{array}$ & $\begin{array}{l}19800000 \\
2090000\end{array}$ & $\begin{array}{l}1710000 \\
1909000\end{array}$ & $\begin{array}{l}633000 \\
778000\end{array}$ & $\begin{array}{l}12500000 \\
1360000\end{array}$ & $\begin{array}{l}857000 \\
1080000\end{array}$ & 0.34 & 0.07 & $\begin{array}{l}0.06 \\
0.07\end{array}$ & $\begin{array}{l}0.02 \\
0.03\end{array}$ & $\begin{array}{l}0.47 \\
0.46\end{array}$ & 0.03 & 0.62 & 1.02 & 20.13 & 6.73 \\
\hline $\begin{array}{l}1172 \\
1172\end{array}$ & $\begin{array}{l}39.98 \\
39.98\end{array}$ & $\begin{array}{l}9550000 \\
14700000\end{array}$ & $\begin{array}{l}20900000 \\
3300000\end{array}$ & $\begin{array}{l}19900000 \\
3500000\end{array}$ & $\begin{array}{l}778000 \\
1120000\end{array}$ & $\begin{array}{l}136000000 \\
24300000\end{array}$ & $\begin{array}{l}10800000 \\
1980000\end{array}$ & $\begin{array}{l}0.33 \\
0.30\end{array}$ & $\begin{array}{l}0.07 \\
0.07\end{array}$ & $\begin{array}{l}0.07 \\
0.07\end{array}$ & $\begin{array}{l}0.03 \\
0.02\end{array}$ & $\begin{array}{l}0.46 \\
0.50\end{array}$ & $\begin{array}{l}0.04 \\
0.04\end{array}$ & $\begin{array}{l}0.65 \\
0.67\end{array}$ & $\begin{array}{l}1.20 \\
0.91\end{array}$ & $\begin{array}{l}19.37 \\
22.11\end{array}$ & $\begin{array}{l}7.51 \\
6.11\end{array}$ \\
\hline 1172 & 40.03 & 9250000 & 2290000 & 2280000 & 669000 & $\begin{array}{l}243000000 \\
1570000\end{array}$ & $\begin{array}{l}19400000 \\
141000\end{array}$ & 0.29 & 0.07 & 0.07 & $\begin{array}{l}0.02 \\
0.02\end{array}$ & 0.50 & $\begin{array}{l}0.04 \\
0.04\end{array}$ & $\begin{array}{l}0.66 \\
0.66\end{array}$ & 0.73 & 24.17 & $\begin{array}{l}6.11 \\
5.07\end{array}$ \\
\hline 1172 & 40.24 & 18100000 & 3360000 & 2430000 & 774000 & 23700000 & 1350000 & 0.36 & 0.07 & 0.05 & 0.02 & 0.48 & 0.03 & $\begin{array}{l}0.58 \\
0.58\end{array}$ & 0.39 & 20.78 & 3.95 \\
\hline 1172 & 40.41 & 72800000 & 17200000 & 14700000 & 5430000 & 100000000 & 7890000 & 0.33 & 0.08 & 0.07 & 0.02 & 0.46 & 0.04 & 0.62 & 1.23 & 19.45 & $\begin{array}{l}3.53 \\
7.54\end{array}$ \\
\hline 1172 & 40.58 & 35400000 & 8210000 & 7280000 & 2260000 & 52200000 & 3900000 & 0.32 & 0.08 & 0.07 & 0.02 & 0.48 & 0.04 & 0.62 & 0.86 & 22.62 & 5.30 \\
\hline 1172 & 40.75 & 50100000 & 10800000 & 8950000 & 3180000 & 66500000 & 4790000 & 0.35 & 0.07 & 0.06 & 0.02 & 0.46 & 0.03 & 0.61 & 1.02 & 21.14 & 6.12 \\
\hline 1172 & 40.91 & 8180000 & 1860000 & 1580000 & 503000 & 11700000 & 875000 & 0.33 & 0.08 & 0.06 & 0.02 & 0.47 & 0.04 & 0.61 & 0.88 & 22.44 & 5.30 \\
\hline 1172 & 41.47 & 32200000 & 7620000 & 7420000 & 2390000 & 49300000 & 4320000 & 0.31 & 0.07 & 0.07 & 0.02 & 0.48 & 0.04 & 0.65 & 1.02 & 21.75 & 6.47 \\
\hline 1172 & 41.81 & 5890000 & 1400000 & 1290000 & 439000 & 8540000 & 676000 & 0.32 & 0.08 & 0.07 & 0.02 & 0.47 & 0.04 & 0.63 & 1.11 & 20.44 & 7.07 \\
\hline 1172 & 42.31 & 38800000 & 8810000 & 7940000 & 2620000 & 56100000 & 4240000 & 0.33 & 0.07 & 0.07 & 0.02 & 0.47 & 0.04 & 0.63 & 0.95 & 21.70 & 5.99 \\
\hline 1172 & 42.73 & 3930000 & 988000 & 994000 & 309000 & 6340000 & 542000 & 0.30 & 0.08 & 0.08 & 0.02 & 0.48 & 0.04 & 0.65 & 1.08 & 21.87 & 6.64 \\
\hline 1172 & 43.07 & 4610000 & 1030000 & 904000 & 259000 & 6660000 & 518000 & 0.33 & 0.07 & 0.06 & 0.02 & 0.48 & 0.04 & 0.62 & 0.52 & 23.49 & 4.52 \\
\hline 1172 & 43.35 & 34400000 & 6950000 & 5650000 & 1870000 & 46700000 & 3010000 & 0.35 & 0.07 & 0.06 & 0.02 & 0.47 & 0.03 & 0.60 & 0.76 & 21.94 & 4.91 \\
\hline 1172 & 43.55 & 4940000 & 1200000 & 1160000 & 351000 & 7740000 & 650000 & 0.31 & 0.07 & 0.07 & 0.02 & 0.48 & 0.04 & 0.64 & 0.96 & 22.88 & 5.70 \\
\hline $\begin{array}{l}1172 \\
1172\end{array}$ & $\begin{array}{l}43.88 \\
43.93\end{array}$ & $\begin{array}{l}60300000 \\
5970000\end{array}$ & $\begin{array}{l}14500000 \\
1450000\end{array}$ & $\begin{array}{l}14400000 \\
1280000\end{array}$ & $\begin{array}{l}467000 \\
412000\end{array}$ & $\begin{array}{l}96440000 \\
8840000\end{array}$ & $\begin{array}{l}831000 \\
650000\end{array}$ & $\begin{array}{l}0.30 \\
0.32\end{array}$ & $\begin{array}{l}0.07 \\
0.08\end{array}$ & $\begin{array}{l}0.07 \\
0.07\end{array}$ & $\begin{array}{l}0.02 \\
0.02\end{array}$ & $\begin{array}{l}0.49 \\
0.48\end{array}$ & 0.04 & 0.65 & $\begin{array}{l}1.02 \\
0.99\end{array}$ & $\begin{array}{l}21.68 \\
21.75\end{array}$ & 6.56 \\
\hline $\begin{array}{l}11172 \\
1172\end{array}$ & $\begin{array}{l}43.93 \\
44.03\end{array}$ & $\begin{array}{l}59900000 \\
3770000\end{array}$ & $\begin{array}{c}14500000 \\
896000\end{array}$ & $\begin{array}{c}12800000 \\
790000\end{array}$ & $\begin{array}{l}4212000 \\
242000\end{array}$ & $\begin{array}{l}88800000 \\
5390000\end{array}$ & $\begin{array}{l}\begin{array}{r}650000 \\
398000\end{array} \\
3\end{array}$ & $\begin{array}{l}0.32 \\
0.33\end{array}$ & $\begin{array}{l}0.08 \\
0.08\end{array}$ & $\begin{array}{l}0.07 \\
0.07\end{array}$ & $\begin{array}{l}0.02 \\
0.02\end{array}$ & $\begin{array}{l}0.48 \\
0.47\end{array}$ & $\begin{array}{l}0.03 \\
0.03\end{array}$ & $\begin{array}{l}0.62 \\
0.61\end{array}$ & $\begin{array}{l}0.99 \\
0.91\end{array}$ & $\begin{array}{l}21.75 \\
22.36\end{array}$ & $\begin{array}{l}6.06 \\
5.63\end{array}$ \\
\hline 1172 & 44.20 & 4430000 & 974000 & 801000 & 245000 & 6340000 & 419000 & 0.34 & 0.07 & 0.06 & 0.02 & 0.48 & 0.03 & 0.60 & 0.60 & 22.55 & $\begin{array}{l}5.63 \\
4.61\end{array}$ \\
\hline 1172 & 44.34 & 19400000 & 4070000 & 3300000 & 1210000 & 27100000 & 1770000 & 0.34 & 0.07 & 0.06 & 0.02 & 0.48 & 0.03 & 0.61 & 0.91 & 21.13 & 5.65 \\
\hline 1172 & 44.44 & 6410000 & 1270000 & 988000 & 348000 & 8170000 & 457000 & 0.36 & 0.07 & 0.06 & 0.02 & 0.46 & 0.03 & 0.59 & 0.98 & 21.12 & 5.29 \\
\hline 1172 & 44.56 & 5510000 & 1260000 & 1110000 & 381000 & 7800000 & 569000 & 0.33 & 0.08 & 0.07 & 0.02 & 0.47 & 0.03 & 0.62 & 1.00 & 20.95 & 6.44 \\
\hline 1172 & 44.66 & 2850000 & 688000 & 637000 & 201000 & 4270000 & 345000 & 0.32 & 0.08 & 0.07 & 0.02 & 0.47 & 0.04 & 0.63 & 1.00 & 22.03 & 6.12 \\
\hline 1172 & 44.81 & 29600000 & 6760000 & 6300000 & 2180000 & 46400000 & 3320000 & 0.31 & 0.07 & 0.07 & 0.02 & 0.49 & 0.04 & 0.64 & 0.87 & 21.13 & 6.18 \\
\hline 1172 & 44.87 & 4540000 & 1000000 & 836000 & 258000 & 6730000 & 440000 & 0.33 & 0.07 & 0.06 & 0.02 & 0.49 & 0.03 & 0.61 & 0.60 & 22.57 & 4.53 \\
\hline 1172 & 44.95 & 1140000 & 255000 & 216000 & 69600 & 1630000 & 115000 & 0.33 & 0.07 & 0.06 & 0.02 & 0.48 & 0.03 & 0.61 & 0.89 & 22.27 & 5.26 \\
\hline 1172 & 45.15 & 33100000 & 7900000 & 7260000 & 2010000 & 51200000 & 4190000 & 0.31 & 0.07 & 0.07 & 0.02 & 0.48 & 0.04 & 0.63 & 0.58 & 24.08 & 4.43 \\
\hline 1172 & 45.28 & 10100000 & 2500000 & 2340000 & 547000 & 16400000 & 1420000 & 0.30 & 0.08 & 0.07 & 0.02 & 0.49 & 0.04 & 0.63 & 0.20 & 24.98 & 3.69 \\
\hline 1172 & 45.44 & 9010000 & 2310000 & 2330000 & 569000 & 15500000 & 1460000 & 0.29 & 0.07 & 0.07 & 0.02 & 0.50 & 0.05 & 0.65 & 0.34 & 25.94 & 3.88 \\
\hline 1172 & 45.50 & 12900000 & 3140000 & 3230000 & 812000 & 22000000 & 2050000 & 0.29 & 0.07 & 0.07 & 0.02 & 0.50 & 0.05 & 0.66 & 0.40 & 25.84 & 3.97 \\
\hline 1172 & $\begin{array}{l}45.52 \\
45.5\end{array}$ & 5214510 & $\begin{array}{l}1315890 \\
25000\end{array}$ & 1480820 & 376629 & 10362200 & 1045540 & 0.26 & 0.07 & 0.07 & 0.02 & 0.52 & 0.05 & 0.69 & 0.29 & 27.07 & 4.00 \\
\hline $\begin{array}{l}1172 \\
1172\end{array}$ & $\begin{array}{l}45.56 \\
45.60\end{array}$ & $\begin{array}{l}98800000 \\
8461870\end{array}$ & $\begin{array}{l}25000000 \\
1989970\end{array}$ & $\begin{array}{l}28660000 \\
2064300\end{array}$ & $\begin{array}{l}8230000 \\
582459\end{array}$ & $\begin{array}{l}19300000 \\
15200900\end{array}$ & $\begin{array}{l}\begin{array}{l}933300000 \\
1354530\end{array} \\
1\end{array}$ & $\begin{array}{l}0.26 \\
0.29\end{array}$ & $\begin{array}{l}0.07 \\
0.07\end{array}$ & $\begin{array}{l}0.08 \\
0.07\end{array}$ & $\begin{array}{l}0.02 \\
0.02\end{array}$ & $\begin{array}{l}0.52 \\
0.51\end{array}$ & $\begin{array}{l}0.05 \\
0.05\end{array}$ & $\begin{array}{l}0.69 \\
0.67\end{array}$ & $\begin{array}{l}0.68 \\
0.53\end{array}$ & $\begin{array}{l}25.15 \\
25.19\end{array}$ & $\begin{array}{l}5.31 \\
4.35\end{array}$ \\
\hline $\begin{array}{l}1172 \\
1172\end{array}$ & $\begin{array}{l}\begin{array}{l}45.60 \\
45.76\end{array} \\
\text {. }\end{array}$ & $\begin{array}{l}8661870 \\
7388080\end{array}$ & $\begin{array}{l}19989970 \\
1724360\end{array}$ & $\begin{array}{l}20643000 \\
1672210\end{array}$ & $\begin{array}{l}582459 \\
456184\end{array}$ & $\begin{array}{l}152023000 \\
13350600\end{array}$ & $\begin{array}{l}\begin{array}{l}33545530 \\
1124530\end{array} \\
1\end{array}$ & $\begin{array}{l}0.29 \\
0.29\end{array}$ & $\begin{array}{l}0.07 \\
0.07\end{array}$ & $\begin{array}{l}0.07 \\
0.07\end{array}$ & $\begin{array}{l}0.02 \\
0.02\end{array}$ & $\begin{array}{l}0.51 \\
0.52\end{array}$ & $\begin{array}{l}0.05 \\
0.04\end{array}$ & $\begin{array}{l}0.67 \\
0.65\end{array}$ & $\begin{array}{l}0.53 \\
0.47\end{array}$ & $\begin{array}{l}25.19 \\
24.99\end{array}$ & $\begin{array}{l}4.35 \\
3.88\end{array}$ \\
\hline 1172 & 45.85 & 19000000 & 4680000 & 5430000 & 1620000 & 34200000 & $\begin{array}{l}\begin{array}{l}11245530 \\
3550000\end{array} \\
3\end{array}$ & 0.28 & 0.07 & $\begin{array}{l}0.07 \\
0.08\end{array}$ & $\begin{array}{l}0.02 \\
0.02\end{array}$ & $\begin{array}{l}0.52 \\
0.50\end{array}$ & $\begin{array}{l}0.04 \\
0.05\end{array}$ & $\begin{array}{l}0.65 \\
0.69\end{array}$ & $\begin{array}{l}0.47 \\
1.02\end{array}$ & $\begin{array}{l}24.99 \\
23.22\end{array}$ & $\begin{array}{l}3.88 \\
6.49\end{array}$ \\
\hline 1172 & 46.08 & 5640000 & 1390000 & 1540000 & 434000 & 10400000 & 1010000 & 0.28 & 0.07 & 0.08 & 0.02 & 0.51 & 0.05 & 0.68 & 0.61 & 25.21 & 4.97 \\
\hline 1172 & 46.19 & 8360000 & 2090000 & 2380000 & 714000 & 15000000 & 1490000 & 0.28 & 0.07 & 0.08 & 0.02 & 0.50 & 0.05 & 0.69 & 1.04 & 23.02 & 6.52 \\
\hline 1172 & 46.42 & 9140000 & 2580000 & 3680000 & 1150000 & 22200000 & 2580000 & 0.22 & 0.06 & 0.09 & 0.03 & 0.54 & 0.06 & . & 1.09 & 22.91 & 7.19 \\
\hline 1172 & 46.76 & 14700000 & 3590000 & 4300000 & 1440000 & 26200000 & 2740000 & 0.28 & 0.07 & 0.08 & 0.03 & 0.49 & 0.05 & 0.70 & 1.49 & 20.15 & 8.08 \\
\hline $\begin{array}{l}1172 \\
1172\end{array}$ & $\begin{array}{l}47.04 \\
47.34\end{array}$ & $\begin{array}{l}31000000 \\
7480000\end{array}$ & $\begin{array}{c}919000 \\
1810000\end{array}$ & $\begin{array}{l}12300000 \\
2220000\end{array}$ & $\begin{array}{l}412000 \\
705000\end{array}$ & $\begin{array}{l}74600000 \\
13500000\end{array}$ & $\begin{array}{l}824000 \\
1440000\end{array}$ & $\begin{array}{l}0.22 \\
0.28\end{array}$ & $\begin{array}{l}0.07 \\
0.07\end{array}$ & $\begin{array}{l}0.09 \\
0.08\end{array}$ & 0.03 & 0.53 & 0.06 & 0.73 & 1.33 & $\begin{array}{l}21.66 \\
21.14\end{array}$ & $\begin{array}{l}7.87 \\
7.65\end{array}$ \\
\hline $\begin{array}{l}1172 \\
1172\end{array}$ & $\begin{array}{l}47.34 \\
47.38\end{array}$ & $\begin{array}{l}74800000 \\
3870000\end{array}$ & $\begin{array}{l}18100000 \\
1070000\end{array}$ & $\begin{array}{l}222200000 \\
1570000\end{array}$ & $\begin{array}{l}7050000 \\
483000\end{array}$ & $\begin{array}{l}135000000 \\
93000000\end{array}$ & 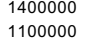 & $\begin{array}{l}0.28 \\
0.22\end{array}$ & $\begin{array}{l}0.07 \\
0.06\end{array}$ & $\begin{array}{l}0.08 \\
0.09\end{array}$ & $\begin{array}{l}0.03 \\
0.03\end{array}$ & $\begin{array}{l}0.50 \\
0.53\end{array}$ & $\begin{array}{l}0.05 \\
0.06\end{array}$ & $\begin{array}{l}0.70 \\
0.75\end{array}$ & $\begin{array}{l}1.36 \\
1.09\end{array}$ & $\begin{array}{l}21.14 \\
22.94\end{array}$ & $\begin{array}{l}7.65 \\
7.20\end{array}$ \\
\hline 1172 & 47.57 & 5610000 & 1630000 & 2240000 & 737000 & 13300000 & 1560000 & 0.22 & 0.06 & 0.09 & 0.03 & 0.53 & 0.06 & 0.74 & $\begin{array}{l}1.39 \\
1.30\end{array}$ & $\begin{array}{l}22.94 \\
21.78\end{array}$ & $\begin{array}{l}7.20 \\
7.84\end{array}$ \\
\hline 1172 & 47.60 & 20600000 & 5760000 & 7910000 & 2620000 & 46500000 & 5320000 & 0.23 & 0.06 & 0.09 & 0.03 & 0.52 & 0.06 & 0.73 & 1.40 & 21.30 & 8.02 \\
\hline 1172 & 47.84 & 2780000 & 691000 & 943 & 325000 & 5390 & 621 & 0.26 & 0.06 & 0.09 & 0.03 & 0.50 & 0.06 & 0.73 & 1.71 & 19.86 & 8.45 \\
\hline 1172 & 47.92 & 2320000 & 688000 & & 304000 & $526 \mathrm{C}$ & & 0.2 & 0.07 & & 0.03 & 0.52 & 0.06 & 0.72 & 1.52 & 20.88 & 8.17 \\
\hline 1172 & $\begin{array}{l}47.96 \\
4807\end{array}$ & & $\begin{array}{l}1100000 \\
147000\end{array}$ & & $\begin{array}{l}485000 \\
77600\end{array}$ & & 851000 & 0.28 & 0.07 & $\begin{array}{l}0.09 \\
0.09\end{array}$ & 0.03 & 0.48 & 0.05 & 0.71 & 1.84 & 18.84 & 8.62 \\
\hline $\begin{array}{l}1172 \\
1172\end{array}$ & $\begin{array}{l}48.07 \\
4811\end{array}$ & 5200000 & $\begin{array}{l}1470000 \\
316000\end{array}$ & $\begin{array}{l}2100000 \\
4770000\end{array}$ & $\begin{array}{l}756000 \\
173000\end{array}$ & 11600000 & $\begin{array}{l}1370000 \\
3190000\end{array}$ & 0.23 & 0.07 & 0.09 & 0.03 & 0.52 & 0.06 & 0.74 & 1.13 & 21.13 & 82 \\
\hline $\begin{array}{l}1172 \\
1172\end{array}$ & $\begin{array}{l}48.11 \\
48.25\end{array}$ & $\begin{array}{r}11100000 \\
140000\end{array}$ & $\begin{array}{r}31600000 \\
46200\end{array}$ & $\begin{array}{l}4770000 \\
65500\end{array}$ & $\begin{array}{r}\begin{array}{r}17330000 \\
23400\end{array} \\
2\end{array}$ & $\begin{array}{r}260000000 \\
333000\end{array}$ & $\begin{array}{l}31900000 \\
42800\end{array}$ & $\begin{array}{l}0.22 \\
0.22\end{array}$ & $\begin{array}{l}0.06 \\
0.07\end{array}$ & $\begin{array}{l}0.10 \\
0.10\end{array}$ & $\begin{array}{l}0.03 \\
0.04\end{array}$ & $\begin{array}{l}0.52 \\
0.51\end{array}$ & $\begin{array}{l}0.06 \\
0.07\end{array}$ & $\begin{array}{l}0.75 \\
0.74\end{array}$ & $\begin{array}{l}0.98 \\
0.87\end{array}$ & $\begin{array}{l}21.69 \\
21.80\end{array}$ & $\begin{array}{l}7.45 \\
7.16\end{array}$ \\
\hline $\begin{array}{l}11 / 2 \\
1172\end{array}$ & $\begin{array}{l}48.25 \\
48.38\end{array}$ & $\begin{array}{l}140000 \\
3640000\end{array}$ & 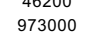 & $\begin{array}{l}655000 \\
1280000\end{array}$ & $\begin{array}{l}23300 \\
496000\end{array}$ & $\begin{array}{l}3333000 \\
7450000\end{array}$ & $\begin{array}{l}488000 \\
870000\end{array}$ & $\begin{array}{l}0.22 \\
0.25\end{array}$ & 0.07 & $\begin{array}{l}0.10 \\
0.09\end{array}$ & $\begin{array}{l}0.04 \\
0.03\end{array}$ & $\begin{array}{l}0.51 \\
0.51\end{array}$ & $\begin{array}{l}0.07 \\
0.06\end{array}$ & $\begin{array}{l}0.14 \\
0.73\end{array}$ & $\begin{array}{l}0.87 \\
1.16\end{array}$ & $\begin{array}{l}21.80 \\
20.62\end{array}$ & $\begin{array}{l}7.16 \\
7.93\end{array}$ \\
\hline 1172 & 48.45 & 4240 & 119 & 178 & 664000 & 10100000 & 1300000 & 0.22 & 0.06 & 0.09 & 0.03 & 0.52 & 0.07 & 0.76 & 1.01 & 21.71 & 7.52 \\
\hline 1172 & 48.58 & 2710 & 765000 & 112 & 435 & $617 C$ & & 0. & 0.06 & 0.09 & 0.04 & 0.51 & 0.07 & 0.75 & 0.81 & 22.20 & 6.97 \\
\hline 1172 & 48.60 & 4370 & 1350 & & 726 & $107 \mathrm{C}$ & 129 & 0. & 0.07 & & 0.04 & 0.53 & 0.06 & 0.74 & 0.77 & 22.44 & 6.81 \\
\hline 1172 & $\begin{array}{l}48.74 \\
483\end{array}$ & 3840000 & 924000 & 1300000 & 511000 & 7150000 & 858000 & 0.26 & 0.06 & 0.09 & 0.04 & 0.49 & 0.06 & 0.74 & 1.13 & 20.37 & 7.89 \\
\hline 1172 & $\begin{array}{l}48.83 \\
48.9\end{array}$ & $\begin{array}{l}7000000 \\
1\end{array}$ & 2080000 & 3180000 & $\begin{array}{l}1170000 \\
287000\end{array}$ & 17100000 & 2180000 & 0.21 & 0.06 & 0.10 & 0.04 & 0.52 & 0.07 & 0.76 & 0.85 & $\begin{array}{l}22.18 \\
21.95\end{array}$ & 7.08 \\
\hline $\begin{array}{l}11172 \\
1172\end{array}$ & $\begin{array}{l}48.89 \\
48.94\end{array}$ & $\begin{array}{l}16100000 \\
4980000\end{array}$ & $\begin{array}{l}485000 \\
1500000\end{array}$ & $\begin{array}{c}794000 \\
2420000\end{array}$ & $\begin{array}{l}287000 \\
9977000\end{array}$ & $\begin{array}{l}4290000 \\
130000\end{array}$ & $\begin{array}{l}616000 \\
1720000\end{array}$ & 0.20 & 0.06 & $\begin{array}{l}0.10 \\
0.10\end{array}$ & $\begin{array}{l}0.04 \\
0.04\end{array}$ & $\begin{array}{l}0.53 \\
0.53\end{array}$ & 0.08 & $\begin{array}{l}0.78 \\
0.77\end{array}$ & $\begin{array}{l}0.96 \\
0.78\end{array}$ & $\begin{array}{l}21.95 \\
22.92\end{array}$ & $\begin{array}{l}7.44 \\
687\end{array}$ \\
\hline 1172 & $\begin{array}{l}48.94 \\
49.11\end{array}$ & 1940000 & 624000 & $\begin{array}{l}244 \\
998\end{array}$ & 399000 & 5310000 & 747000 & $\begin{array}{l}0.19 \\
0.19\end{array}$ & 0.06 & 0.10 & $\begin{array}{l}0.04 \\
0.04\end{array}$ & 0.53 & 0.07 & 0.77 & 0.95 & 22.89 & $\begin{array}{l}6.81 \\
7.30\end{array}$ \\
\hline 1172 & 49.23 & 1267260 & 408540 & 679 & 243481 & 4065090 & 578 & 0.17 & 0.06 & 0.09 & 0.03 & 0.56 & 0.08 & 0.79 & 1.23 & 22.21 & 7.86 \\
\hline 1172 & 49.28 & 3550000 & 1040000 & 979000 & 382000 & 5050000 & 782000 & 0.30 & 0.09 & 0.08 & 0.03 & 0.43 & 0.07 & 0.67 & 1.92 & 17.22 & 8.81 \\
\hline 1172 & 49.29 & 2251630 & 443786 & 794478 & 314423 & 4595120 & 697795 & 0.25 & 0.05 & 0.09 & 0.03 & 0.51 & 0.08 & 0.80 & 1.34 & 19.95 & 8.30 \\
\hline
\end{tabular}


Eocene dataset for OPTiMAL

\begin{tabular}{|c|c|c|c|c|c|c|c|c|c|c|c|c|c|c|c|c|c|}
\hline SITE & AGE & $\begin{array}{c}1302 \\
\text { GDGTO } \\
\end{array}$ & $\begin{array}{r}1300 \\
\text { GDGT1 } \\
\end{array}$ & $\begin{array}{c}1298 \\
\text { GDGT2 }\end{array}$ & $\begin{array}{c}1296 \\
\text { GDGT3 } \\
\end{array}$ & $\begin{array}{l}1292 \\
\text { CREN } \\
\end{array}$ & $\begin{array}{l}1292^{\prime} \\
\text { CREN' }\end{array}$ & Fr. 1302 & Fr. 1300 & Fr.1298 & Fr.1296 & Fr.1292 & Fr.1292' & $\begin{array}{c}\text { TEX } \\
\text { (published) }\end{array}$ & Innarest & $\begin{array}{c}\text { OPTIMAL } \\
\text { SST }\end{array}$ & $\begin{array}{c}\text { OPTTMALAL } \\
\text { StDev }\end{array}$ \\
\hline 1172 & 49.30 & 3240000 & 1090000 & 1740000 & 679000 & 9710000 & 1270000 & 0.18 & 0.06 & 0.10 & 0.04 & 0.55 & 0.07 & 0.77 & 0.79 & 23.18 & 6.87 \\
\hline 1172 & 49.43 & 2470000 & 892000 & 1390000 & 566000 & 7370000 & 1160000 & 0.18 & 0.06 & 0.10 & 0.04 & 0.53 & 0.08 & 0.78 & 1.17 & 22.22 & 7.77 \\
\hline 1172 & 49.65 & 6430000 & 1960000 & 2960000 & 1180000 & 159000000 & 2090000 & 0.21 & 0.06 & 0.10 & 0.04 & 0.52 & 0.07 & 0.76 & 0.79 & 22.96 & 6.88 \\
\hline 1172 & 50.09 & 2060000 & 614000 & 1070000 & 418000 & 6500000 & 1010000 & 0.18 & 0.05 & 0.09 & 0.04 & 0.56 & 0.09 & 0.80 & 1.09 & 21.71 & 7.80 \\
\hline 1172 & 50.16 & 5240000 & 1360000 & 2290000 & 1010000 & 11700000 & 1610000 & 0.23 & 0.06 & 0.10 & 0.04 & 0.50 & 0.07 & 0.78 & 1.21 & 21.69 & 7.98 \\
\hline 1172 & 50.33 & 2190000 & 632000 & 1010000 & 376000 & 5970000 & 777000 & 0.20 & 0.06 & 0.09 & 0.03 & 0.54 & 0.07 & 0.77 & 1.03 & 22.12 & 7.55 \\
\hline 1172 & 50.63 & 544000 & 175000 & 288000 & 119000 & 1480000 & 224000 & 0.19 & 0.06 & 0.10 & 0.04 & 0.52 & 0.08 & 0.78 & 1.26 & 22.26 & 7.83 \\
\hline 1172 & 50.78 & 3870000 & 836000 & 1330000 & 601000 & 7540000 & 1020000 & 0.25 & 0.06 & 0.09 & 0.04 & 0.50 & 0.07 & 0.78 & 1.12 & 21.33 & 7.85 \\
\hline 1172 & $\begin{array}{l}51.41 \\
52.74\end{array}$ & $\begin{array}{l}4190000 \\
147000\end{array}$ & 1060000 & 1620000 & 753000 & 8560000 & 1160000 & 0.24 & 0.06 & 0.09 & 0.04 & 0.49 & 0.07 & 0.77 & 1.30 & 21.27 & 8.12 \\
\hline $\begin{array}{l}1172 \\
1172\end{array}$ & $\begin{array}{l}52.74 \\
53.08\end{array}$ & $\begin{array}{l}\begin{array}{l}14700000 \\
1520000\end{array} \\
0\end{array}$ & $\begin{array}{l}487000 \\
535000\end{array}$ & $\begin{array}{l}639000 \\
865000\end{array}$ & $\begin{array}{l}2790000 \\
426000\end{array}$ & $\begin{array}{l}\begin{array}{l}296600000 \\
4460000\end{array} \\
4\end{array}$ & $\begin{array}{l}398000 \\
630000\end{array}$ & $\begin{array}{l}0.24 \\
0.18\end{array}$ & $\begin{array}{l}0.08 \\
0.06\end{array}$ & 0.10 & $\begin{array}{l}0.04 \\
0.05\end{array}$ & $\begin{array}{l}0.47 \\
0.53\end{array}$ & $\begin{array}{l}0.06 \\
0.07\end{array}$ & $\begin{array}{l}0.73 \\
0.78\end{array}$ & $\begin{array}{l}1.60 \\
1.05\end{array}$ & 19.72 & 8.51 \\
\hline $\begin{array}{l}1172 \\
1172\end{array}$ & $\begin{array}{l}53.08 \\
53.19\end{array}$ & $\begin{array}{l}\begin{array}{l}15200000 \\
342000\end{array} \\
\end{array}$ & $\begin{array}{l}5350000 \\
741000\end{array}$ & $\begin{array}{l}865000 \\
1180000\end{array}$ & $\begin{array}{l}4266000 \\
537000\end{array}$ & $\begin{array}{l}\begin{array}{l}446600000 \\
5860000\end{array} \\
5\end{array}$ & $\begin{array}{l}631000 \\
804000\end{array}$ & $\begin{array}{l}0.18 \\
0.04\end{array}$ & $\begin{array}{l}0.06 \\
0.08\end{array}$ & $\begin{array}{l}0.10 \\
0.12\end{array}$ & $\begin{array}{l}0.05 \\
0.06\end{array}$ & $\begin{array}{l}0.53 \\
0.62\end{array}$ & $\begin{array}{l}0.07 \\
0.08\end{array}$ & $\begin{array}{l}0.78 \\
0.77\end{array}$ & $\begin{array}{l}1.05 \\
1.90\end{array}$ & $\begin{array}{l}21.54 \\
1720\end{array}$ & $\begin{array}{l}7.74 \\
8.82\end{array}$ \\
\hline 1172 & $\begin{array}{l}53.53 \\
53.19\end{array}$ & $\begin{array}{l}\begin{array}{r}342000 \\
6190000\end{array} \\
6\end{array}$ & $\begin{array}{l}7417000 \\
1790000\end{array}$ & $\begin{array}{l}1800000 \\
2450000\end{array}$ & $\begin{array}{l}537000 \\
915000\end{array}$ & $\begin{array}{l}586600000 \\
14800000\end{array}$ & $\begin{array}{l}8044000 \\
1570000\end{array}$ & $\begin{array}{l}0.04 \\
0.22\end{array}$ & $\begin{array}{l}0.08 \\
0.06\end{array}$ & $\begin{array}{l}0.12 \\
0.09\end{array}$ & $\begin{array}{l}0.06 \\
0.03\end{array}$ & $\begin{array}{l}0.62 \\
0.53\end{array}$ & $\begin{array}{l}0.08 \\
0.06\end{array}$ & $\begin{array}{l}0.77 \\
0.73\end{array}$ & $\begin{array}{l}1.90 \\
1.15\end{array}$ & $\begin{array}{l}17.20 \\
21.43\end{array}$ & $\begin{array}{l}8.82 \\
7.79\end{array}$ \\
\hline 1172 & 53.63 & 4880000 & 1540000 & 2540000 & 1170000 & 13800000 & 1710000 & $\begin{array}{l}0.19 \\
0.19\end{array}$ & 0.06 & 0.10 & 0.05 & 0.54 & 0.07 & 0.78 & 1.04 & $\begin{array}{l}21.45 \\
22.92\end{array}$ & $\begin{array}{l}7.19 \\
7.44\end{array}$ \\
\hline 1172 & 53.99 & 2480000 & 758000 & 1080000 & 486000 & 6080000 & 704000 & 0.21 & 0.07 & 0.09 & 0.04 & 0.52 & 0.06 & 0.75 & 1.12 & 23.30 & 7.39 \\
\hline 1172 & 54.06 & 2480000 & 759000 & 1030000 & 396000 & 5960000 & 627000 & 0.22 & 0.07 & 0.09 & 0.04 & 0.53 & 0.06 & 0.73 & 0.79 & 22.29 & 6.86 \\
\hline 1172 & 54.17 & 2480000 & 660000 & 794000 & 257000 & 5750000 & 546000 & 0.24 & 0.06 & 0.08 & 0.02 & 0.55 & 0.05 & 0.71 & 0.92 & 2396 & 619 \\
\hline 1172 & 54.29 & 1110000 & 333000 & 425000 & 142000 & 2870000 & 311000 & 0.21 & 0.06 & 0.08 & 0.03 & 0.55 & 0.06 & 0.73 & 1.00 & 23.21 & 6.83 \\
\hline 1172 & 54.44 & 2320000 & 623000 & 692000 & 214000 & 3930000 & 432000 & 0.28 & 0.08 & 0.08 & 0.03 & 0.48 & 0.05 & 0.68 & 1.48 & 20.72 & 7.88 \\
\hline 1172 & 54.63 & 3880000 & 862000 & 976000 & 271000 & 6930000 & 632000 & 0.29 & 0.06 & 0.07 & 0.02 & 0.51 & 0.05 & 0.69 & 0.60 & 25.12 & 4.70 \\
\hline 1172 & 54.77 & 3730000 & 861000 & 816000 & 229000 & 6650000 & 496000 & 0.29 & 0.07 & 0.06 & 0.02 & 0.52 & 0.04 & 0.64 & 0.49 & 24.26 & 3.87 \\
\hline 1172 & 54.87 & 1640000 & 414000 & 426000 & 122000 & 3180000 & 262000 & 0.27 & 0.07 & 0.07 & 0.02 & 0.53 & 0.04 & 0.66 & 0.45 & 25.36 & 4.24 \\
\hline 1172 & 54.98 & 4070000 & 881000 & 801000 & 210000 & 6230000 & 447000 & 0.32 & 0.07 & 0.06 & 0.02 & 0.49 & 0.04 & 0.62 & 0.30 & 23.82 & 3.82 \\
\hline 1172 & 55.10 & 3850000 & 969000 & 1020000 & 326000 & 7450000 & 672000 & 0.27 & 0.07 & 0.07 & 0.02 & 0.52 & 0.05 & 0.68 & 0.82 & 23.78 & 5.67 \\
\hline 1172 & 55.30 & 6040000 & 1290000 & 1120000 & 344000 & 9210000 & 599000 & 0.32 & 0.07 & 0.06 & 0.02 & 0.50 & 0.03 & 0.62 & 0.58 & 22.70 & 4.37 \\
\hline 1172 & 55.56 & 2030000 & 409000 & 359000 & 98500 & 3030000 & 208000 & 0.33 & 0.07 & 0.06 & 0.02 & 0.49 & 0.03 & 0.62 & 0.43 & 23.17 & 3.83 \\
\hline 1172 & 55.68 & 4100000 & 837000 & 781000 & 203000 & 6320000 & 460000 & 0.32 & 0.07 & 0.06 & 0.02 & 0.50 & 0.04 & 0.63 & 0.40 & 23.84 & 3.78 \\
\hline 1172 & 55.86 & 1780000 & 357000 & 337000 & 86200 & 2700000 & 192000 & 0.33 & 0.07 & 0.06 & 0.02 & 0.50 & 0.04 & 0.63 & 0.39 & 23.85 & 3.77 \\
\hline 1172 & 56.01 & 5550000 & 1170000 & 1050000 & 260000 & 8570000 & 592000 & 0.32 & 0.07 & 0.06 & 0.02 & 0.50 & 0.03 & 0.62 & 0.25 & 23.38 & 3.60 \\
\hline 1172 & 56.24 & 1580000 & 313000 & 320000 & 88800 & 2440000 & 207000 & 0.32 & 0.06 & 0.06 & 0.02 & 0.49 & 0.04 & 0.66 & 0.52 & 24.40 & 4.42 \\
\hline 1172 & 56.37 & 1830000 & 358000 & 320000 & 87000 & 2650000 & 181000 & 0.34 & 0.07 & 0.06 & 0.02 & 0.49 & 0.03 & 0.62 & 0.47 & 23.26 & 3.89 \\
\hline 1172 & 56.84 & 1180000 & 253000 & 216000 & 64400 & 2080000 & 143000 & 0.30 & 0.06 & 0.05 & 0.02 & 0.53 & 0.04 & 0.63 & 0.46 & 22.73 & 3.77 \\
\hline 1172 & 55.29 & 0.00 & 0.09 & 0.09 & 0.03 & 0.73 & 0.05 & 0.00 & 0.09 & 0.09 & 0.03 & 0.73 & 0.05 & 0.65 & 2.02 & 16.26 & 8.83 \\
\hline $\begin{array}{l}1172 \\
1177\end{array}$ & $\begin{array}{l}55.31 \\
55.33\end{array}$ & 0.29 & 0.07 & $\begin{array}{l}0.06 \\
0.09\end{array}$ & 0.02 & 0.52 & 0.04 & 0.29 & $\begin{array}{l}0.07 \\
0.09\end{array}$ & 0.06 & 0.02 & 0.52 & 0.04 & 0.65 & 0.57 & $\begin{array}{l}24.24 \\
1625\end{array}$ & $\begin{array}{l}4.07 \\
8.85\end{array}$ \\
\hline $\begin{array}{l}1172 \\
1172\end{array}$ & $\begin{array}{r}55.33 \\
55.34\end{array}$ & $\begin{array}{l}0.00 \\
0.00\end{array}$ & $\begin{array}{l}0.09 \\
0.10\end{array}$ & $\begin{array}{l}0.09 \\
0.09\end{array}$ & $\begin{array}{l}0.03 \\
0.03\end{array}$ & $\begin{array}{l}0.73 \\
0.73\end{array}$ & $\begin{array}{l}0.05 \\
0.05\end{array}$ & $\begin{array}{l}0.00 \\
0.00\end{array}$ & $\begin{array}{l}0.09 \\
0.10\end{array}$ & $\begin{array}{l}0.09 \\
0.099\end{array}$ & $\begin{array}{l}0.03 \\
0.03\end{array}$ & $\begin{array}{l}0.73 \\
0.73\end{array}$ & $\begin{array}{l}0.05 \\
0.05\end{array}$ & $\begin{array}{l}0.65 \\
0.64\end{array}$ & $\begin{array}{l}2.12 \\
2.07\end{array}$ & $\begin{array}{l}16.25 \\
16.14\end{array}$ & $\begin{array}{l}8.85 \\
8.84\end{array}$ \\
\hline $\begin{array}{l}11122 \\
1172\end{array}$ & $\begin{array}{l}55.34 \\
55.35\end{array}$ & $\begin{array}{l}0.00 \\
0.00\end{array}$ & $\begin{array}{l}0.10 \\
0.09\end{array}$ & $\begin{array}{l}0.09 \\
0.09\end{array}$ & $\begin{array}{l}0.03 \\
0.03\end{array}$ & $\begin{array}{l}0.73 \\
0.73\end{array}$ & $\begin{array}{l}0.05 \\
0.06\end{array}$ & $\begin{array}{l}0.00 \\
0.00\end{array}$ & $\begin{array}{l}0.10 \\
0.09\end{array}$ & $\begin{array}{l}0.099 \\
0.09\end{array}$ & $\begin{array}{l}0.03 \\
0.03\end{array}$ & $\begin{array}{l}0.73 \\
0.73\end{array}$ & $\begin{array}{l}0.05 \\
0.06\end{array}$ & $\begin{array}{l}0.64 \\
0.65\end{array}$ & $\begin{array}{l}2.07 \\
2.09\end{array}$ & $\begin{array}{l}16.14 \\
16.26\end{array}$ & $\begin{array}{l}8.84 \\
8.85\end{array}$ \\
\hline 1172 & 55.36 & 0.27 & 0.07 & $\begin{array}{l}0.09 \\
0.07\end{array}$ & $\begin{array}{l}0.03 \\
0.02\end{array}$ & $\begin{array}{l}0.13 \\
0.54\end{array}$ & $\begin{array}{l}0.06 \\
0.04\end{array}$ & $\begin{array}{l}0.00 \\
0.27\end{array}$ & 0.07 & $\begin{array}{l}0.09 \\
0.07\end{array}$ & $\begin{array}{l}0.03 \\
0.02\end{array}$ & 0.54 & $\begin{array}{l}0.06 \\
0.04\end{array}$ & $\begin{array}{l}0.05 \\
0.66\end{array}$ & $\begin{array}{l}2.09 \\
0.48\end{array}$ & $\begin{array}{l}\begin{array}{l}26.26 \\
24.92\end{array}\end{array}$ & $\begin{array}{l}8.85 \\
4.41\end{array}$ \\
\hline 1172 & 55.38 & 0.29 & 0.07 & 0.06 & 0.02 & 0.52 & 0.04 & 0.29 & 0.07 & 0.06 & 0.02 & 0.52 & 0.04 & 0.64 & 0.64 & 23.71 & 4.22 \\
\hline 1172 & 55.39 & 0.31 & 0.07 & 0.06 & 0.02 & 0.51 & 0.03 & 0.31 & 0.07 & 0.06 & 0.02 & 0.51 & 0.03 & 0.63 & 0.64 & 22.90 & 4.28 \\
\hline 1172 & 55.41 & 0.27 & 0.06 & 0.07 & 0.02 & 0.53 & 0.04 & 0.27 & 0.06 & 0.07 & 0.02 & 0.53 & 0.04 & 0.67 & 0.67 & 24.00 & 5.04 \\
\hline 1172 & 55.43 & 0.27 & 0.06 & 0.06 & 0.02 & 0.54 & 0.04 & 0.27 & 0.06 & 0.06 & 0.02 & 0.54 & 0.04 & 0.67 & 0.63 & 24.11 & 4.89 \\
\hline 1172 & 55.45 & 0.27 & 0.06 & 0.07 & 0.02 & 0.53 & 0.05 & 0.27 & 0.06 & 0.07 & 0.02 & 0.53 & 0.05 & 0.68 & 0.70 & 24.02 & 5.24 \\
\hline 1172 & 55.45 & 0.26 & 0.06 & 0.07 & 0.02 & 0.54 & 0.05 & 0.26 & 0.06 & 0.07 & 0.02 & 0.54 & 0.05 & 0.69 & 0.65 & 24.71 & 5.19 \\
\hline 1172 & 55.47 & 0.23 & 0.06 & 0.07 & 0.02 & 0.55 & 0.06 & 0.23 & 0.06 & 0.07 & 0.02 & 0.55 & 0.06 & 0.73 & 0.96 & 23.69 & 6.47 \\
\hline 1172 & 55.48 & 0.18 & 0.06 & 0.08 & 0.03 & 0.59 & 0.07 & 0.18 & 0.06 & 0.08 & 0.03 & 0.59 & 0.07 & 0.76 & 0.63 & 24.88 & 5.65 \\
\hline 1172 & 55.49 & 0.18 & 0.06 & 0.07 & 0.03 & 0.59 & 0.07 & 0.18 & 0.06 & 0.07 & 0.03 & 0.59 & 0.07 & 0.76 & 0.74 & 24.35 & 6.11 \\
\hline 1172 & 55.50 & 0.16 & 0.05 & 0.07 & 0.03 & 0.60 & 0.08 & 0.16 & 0.05 & 0.07 & 0.03 & 0.60 & 0.08 & 0.79 & 0.87 & 24.47 & 6.45 \\
\hline 1172 & 55.51 & 0.11 & 0.04 & 0.07 & 0.03 & 0.65 & 0.09 & 0.11 & 0.04 & 0.07 & 0.03 & 0.65 & 0.09 & 0.82 & 1.06 & 23.61 & 7.13 \\
\hline 1172 & 55.52 & 0.12 & 0.05 & 0.08 & 0.03 & 0.63 & 0.09 & 0.12 & 0.05 & 0.08 & 0.03 & 0.63 & 0.09 & 0.81 & 1.12 & 23.84 & 7.09 \\
\hline 1172 & 55.53 & 0.15 & 0.05 & 0.07 & 0.03 & 0.61 & 0.09 & 0.15 & 0.05 & 0.07 & 0.03 & 0.61 & 0.09 & 0.80 & 0.94 & 24.43 & 6.69 \\
\hline 1172 & 55.53 & 0.16 & 0.05 & 0.07 & 0.03 & 0.61 & 0.08 & 0.16 & 0.05 & 0.07 & 0.03 & 0.61 & 0.08 & 0.78 & 0.89 & 24.30 & 6.56 \\
\hline 1172 & 55.53 & 0.62 & 0.02 & 0.03 & 0.01 & 0.28 & 0.04 & 0.62 & 0.02 & 0.03 & 0.01 & 0.28 & 0.04 & 0.78 & 1.33 & 10.15 & 7.93 \\
\hline 1172 & 55.54 & 0.00 & 0.07 & 0.09 & 0.03 & 0.73 & 0.08 & 0.00 & 0.07 & 0.09 & 0.03 & 0.73 & 0.08 & 0.75 & 1.88 & 17.60 & 8.75 \\
\hline 1172 & 55.55 & 0.33 & 0.05 & 0.06 & 0.02 & 0.49 & 0.06 & 0.33 & 0.05 & 0.06 & 0.02 & 0.49 & 0.06 & 0.75 & 1.27 & 20.69 & 7.32 \\
\hline 1172 & 55.55 & 0.31 & 0.06 & 0.06 & 0.02 & 0.51 & 0.04 & 0.31 & 0.06 & 0.06 & 0.02 & 0.51 & 0.04 & 0.63 & 0.40 & 22.88 & 3.82 \\
\hline 1172 & 55.56 & 0.30 & 0.06 & 0.06 & 0.02 & 0.53 & 0.04 & 0.30 & 0.06 & 0.06 & 0.02 & 0.53 & 0.04 & 0.66 & 0.61 & 23.92 & 4.26 \\
\hline 1172 & $\begin{array}{l}55.56 \\
55.57\end{array}$ & 0.31 & 0.06 & 0.06 & 0.02 & 0.52 & 0.04 & 0.31 & $\begin{array}{l}0.06 \\
0.06\end{array}$ & 0.06 & 0.02 & 0.52 & 0.04 & 0.64 & 0.43 & 23.10 & 3.90 \\
\hline $\begin{array}{l}1172 \\
1172\end{array}$ & $\begin{array}{l}55.57 \\
55.57\end{array}$ & $\begin{array}{l}0.29 \\
0.30\end{array}$ & $\begin{array}{l}0.06 \\
0.06\end{array}$ & 0.06 & 0.02 & $\begin{array}{l}0.52 \\
0.51\end{array}$ & 0.04 & 0.29 & $\begin{array}{l}0.06 \\
0.06\end{array}$ & 0.06 & 0.02 & $\begin{array}{l}0.52 \\
0.51\end{array}$ & $\begin{array}{l}0.04 \\
0.04\end{array}$ & $\begin{array}{l}0.66 \\
0.66\end{array}$ & $\begin{array}{l}0.52 \\
0.52\end{array}$ & $\begin{array}{l}24.52 \\
24.59\end{array}$ & 3.90 \\
\hline $\begin{array}{l}1172 \\
1172\end{array}$ & $\begin{array}{l}55.57 \\
55.58\end{array}$ & $\begin{array}{l}0.30 \\
0.28\end{array}$ & $\begin{array}{l}0.06 \\
0.06\end{array}$ & $\begin{array}{l}0.06 \\
0.06\end{array}$ & $\begin{array}{l}0.02 \\
0.02\end{array}$ & $\begin{array}{l}0.51 \\
0.53\end{array}$ & $\begin{array}{l}0.04 \\
0.05\end{array}$ & $\begin{array}{l}0.30 \\
0.28\end{array}$ & $\begin{array}{l}0.06 \\
0.06\end{array}$ & $\begin{array}{l}0.06 \\
0.06\end{array}$ & $\begin{array}{l}0.02 \\
0.02\end{array}$ & $\begin{array}{l}0.51 \\
0.53\end{array}$ & $\begin{array}{l}0.04 \\
0.05\end{array}$ & $\begin{array}{l}0.66 \\
0.67\end{array}$ & $\begin{array}{l}0.57 \\
0.62\end{array}$ & $\begin{array}{l}24.59 \\
24.49\end{array}$ & $\begin{array}{l}4.13 \\
4.59\end{array}$ \\
\hline $\begin{array}{l}1172 \\
1172 \\
\end{array}$ & $\begin{array}{l}55.58 \\
55.58\end{array}$ & $\begin{array}{l}0.28 \\
0.32\end{array}$ & $\begin{array}{l}0.06 \\
0.06\end{array}$ & $\begin{array}{l}0.06 \\
0.06\end{array}$ & $\begin{array}{l}0.02 \\
0.02\end{array}$ & $\begin{array}{l}0.33 \\
0.49\end{array}$ & $\begin{array}{l}0.05 \\
0.05\end{array}$ & $\begin{array}{l}0.28 \\
0.32\end{array}$ & $\begin{array}{l}0.06 \\
0.06\end{array}$ & $\begin{array}{l}0.06 \\
0.06\end{array}$ & $\begin{array}{l}0.02 \\
0.02\end{array}$ & $\begin{array}{l}0.53 \\
0.49\end{array}$ & $\begin{array}{l}0.05 \\
0.05\end{array}$ & 0.69 & $\begin{array}{l}0.02 \\
0.87\end{array}$ & 23.30 & 5.47 \\
\hline 1172 & 55.59 & 0.27 & 0.07 & 0.07 & 0.02 & 0.53 & 0.05 & 0.27 & 0.07 & 0.07 & 0.02 & 0.53 & 0.05 & 0.66 & 0.46 & 25.22 & 4.28 \\
\hline 1172 & 55.59 & 0.28 & 0.06 & 0.06 & 0.02 & 0.53 & 0.05 & 0.28 & 0.06 & 0.06 & 0.02 & 0.53 & 0.05 & 0.68 & 0.61 & 24.65 & 4.71 \\
\hline 1172 & 55.60 & 0.28 & 0.06 & 0.06 & 0.02 & 0.53 & 0.04 & 0.28 & 0.06 & 0.06 & 0.02 & 0.53 & 0.04 & 0.67 & 0.46 & 24.90 & 4.05 \\
\hline 1172 & 55.63 & 0.31 & 0.06 & 0.06 & 0.02 & 0.50 & 0.04 & 0.31 & 0.06 & 0.06 & 0.02 & 0.50 & 0.04 & 0.66 & 0.64 & 23.75 & 4.48 \\
\hline $\begin{array}{l}1172 \\
11172\end{array}$ & $\begin{array}{l}55.66 \\
55.67\end{array}$ & 0.28 & $\begin{array}{l}0.06 \\
0.06\end{array}$ & $\begin{array}{l}0.06 \\
0.06\end{array}$ & 0.02 & $\begin{array}{l}0.53 \\
0.53\end{array}$ & 0.04 & 0.28 & 0.06 & 0.06 & 0.02 & 0.53 & 0.04 & 0.66 & 0.49 & $\begin{array}{l}24.80 \\
2.80\end{array}$ & 3.86 \\
\hline $\begin{array}{l}1172 \\
1172\end{array}$ & 55.67 & $\begin{array}{l}0.28 \\
0.31\end{array}$ & $\begin{array}{l}0.06 \\
0.06\end{array}$ & $\begin{array}{l}0.06 \\
0.06\end{array}$ & $\begin{array}{l}0.02 \\
0.02\end{array}$ & $\begin{array}{l}0.53 \\
0.51\end{array}$ & $\begin{array}{l}0.04 \\
0.04\end{array}$ & $\begin{array}{l}0.28 \\
0.31\end{array}$ & $\begin{array}{l}0.06 \\
0.06\end{array}$ & $\begin{array}{l}0.06 \\
0.066\end{array}$ & $\begin{array}{l}0.02 \\
0.02\end{array}$ & $\begin{array}{l}0.53 \\
0.51\end{array}$ & 0.04 & 0.66 & 0.51 & $\begin{array}{l}24.67 \\
2387\end{array}$ & $\begin{array}{l}3.86 \\
3.93\end{array}$ \\
\hline $\begin{array}{l}111 / 2 \\
1172\end{array}$ & $\begin{array}{l}55.68 \\
55.69\end{array}$ & $\begin{array}{l}0.31 \\
0.28\end{array}$ & $\begin{array}{l}0.06 \\
0.06\end{array}$ & $\begin{array}{l}0.06 \\
0.06\end{array}$ & $\begin{array}{l}0.02 \\
0.02\end{array}$ & $\begin{array}{l}0.51 \\
0.53\end{array}$ & $\begin{array}{l}0.04 \\
0.04\end{array}$ & $\begin{array}{l}0.31 \\
0.28\end{array}$ & $\begin{array}{l}0.06 \\
0.06\end{array}$ & $\begin{array}{l}0.06 \\
0.06\end{array}$ & $\begin{array}{l}0.02 \\
0.02\end{array}$ & $\begin{array}{l}0.51 \\
0.53\end{array}$ & $\begin{array}{l}0.04 \\
0.04\end{array}$ & $\begin{array}{l}0.65 \\
0.65\end{array}$ & $\begin{array}{l}0.49 \\
0.48\end{array}$ & $\begin{array}{l}23.82 \\
24.48\end{array}$ & $\begin{array}{l}3.93 \\
3.81\end{array}$ \\
\hline
\end{tabular}


Eocene dataset for OPTiMAL

\begin{tabular}{|c|c|c|c|c|c|c|c|c|c|c|c|c|c|c|c|c|c|}
\hline SITE & AGE & $\begin{array}{r}1302 \\
\text { GDGTO }\end{array}$ & $\begin{array}{c}1300 \\
\text { GDGT1 }\end{array}$ & $\begin{array}{c}1298 \\
\text { GDGT2 }\end{array}$ & $\begin{array}{l}1296 \\
\text { GDGT3 }\end{array}$ & $\begin{array}{l}1292 \\
\text { CREN }\end{array}$ & $\begin{array}{l}1292^{\prime} \\
\text { CREN' }\end{array}$ & Fr. 1302 & Fr. 1300 & Fr. 1298 & Fr. 1296 & Fr. 1292 & Fr.1292' & $\begin{array}{c}\text { TEX } \\
\text { (published) }\end{array}$ & & $\begin{array}{c}\text { OPTIMAL } \\
\text { SST }\end{array}$ & $\begin{array}{c}\text { OPTTMAL } \\
\text { StDev }\end{array}$ \\
\hline 1172 & 55.71 & 0.44 & 0.05 & 0.05 & 0.01 & 0.41 & 0.03 & 0.44 & 0.05 & 0.05 & 0.01 & 0.41 & 0.03 & 0.65 & 0.87 & 13.54 & 5.35 \\
\hline 1172 & 55.72 & 0.29 & 0.06 & 0.06 & 0.02 & 0.53 & 0.04 & 0.29 & 0.06 & 0.06 & 0.02 & 0.53 & 0.04 & 0.65 & 0.58 & 24.07 & \\
\hline 1172 & 55.73 & 0.30 & 0.06 & 0.06 & 0.02 & 0.52 & 0.04 & 0.30 & 0.06 & 0.06 & 0.02 & 0.52 & 0.04 & 0.64 & 0.47 & 23.62 & 3.74 \\
\hline 1172 & 55.76 & 0.31 & 0.06 & 0.06 & 0.02 & 0.52 & 0.04 & 0.31 & 0.06 & 0.06 & 0.02 & 0.52 & 0.04 & 0.63 & 0.47 & 23.48 & 3.75 \\
\hline 1172 & 55.78 & 0.31 & 0.06 & 0.06 & 0.02 & 0.52 & 0.03 & 0.31 & 0.06 & 0.06 & 0.02 & 0.52 & 0.03 & 0.63 & 0.35 & 22.76 & 3.66 \\
\hline 1172 & 55.81 & 0.30 & 0.06 & 0.06 & 0.02 & 0.53 & 0.04 & 0.30 & 0.06 & 0.06 & 0.02 & 0.53 & 0.04 & 0.64 & 0.49 & 23.55 & 3.77 \\
\hline 1172 & 55.86 & 0.29 & 0.06 & 0.06 & 0.02 & 0.53 & 0.04 & 0.29 & 0.06 & 0.06 & 0.02 & 0.53 & 0.04 & 0.64 & 0.36 & 23.80 & 3.61 \\
\hline 1172 & 55.91 & 0.30 & 0.06 & 0.06 & 0.02 & 0.53 & 0.04 & 0.30 & 0.06 & 0.06 & 0.02 & 0.53 & 0.04 & 0.63 & 0.33 & 22.86 & 3.60 \\
\hline $\begin{array}{l}1172 \\
1172\end{array}$ & 55.96 & 0.28 & 0.06 & 0.06 & 0.02 & 0.54 & 0.04 & 0.28 & 0.06 & 0.06 & 0.02 & 0.54 & 0.04 & 0.64 & 0.40 & 23.98 & 3.71 \\
\hline $\begin{array}{l}1172 \\
1172\end{array}$ & $\begin{array}{l}56.01 \\
56.06\end{array}$ & $\begin{array}{l}0.31 \\
0.37\end{array}$ & $\begin{array}{l}0.06 \\
0.06\end{array}$ & $\begin{array}{l}0.06 \\
0.06\end{array}$ & $\begin{array}{l}0.01 \\
0.01\end{array}$ & $\begin{array}{l}0.51 \\
0.46\end{array}$ & $\begin{array}{l}0.03 \\
0.03\end{array}$ & $\begin{array}{l}0.31 \\
0.37\end{array}$ & 0.06 & $\begin{array}{l}0.06 \\
0.06\end{array}$ & 0.01 & $\begin{array}{l}0.51 \\
0.46\end{array}$ & 0.03 & $\begin{array}{l}0.63 \\
0.64\end{array}$ & 0.21 & $\begin{array}{l}22.98 \\
22.39\end{array}$ & 3.55 \\
\hline $\begin{array}{l}1172 \\
1172\end{array}$ & $\begin{array}{l}56.06 \\
56.11\end{array}$ & $\begin{array}{l}0.37 \\
0.24\end{array}$ & $\begin{array}{l}0.06 \\
0.06\end{array}$ & $\begin{array}{l}0.06 \\
0.07\end{array}$ & $\begin{array}{l}0.01 \\
0.02\end{array}$ & $\begin{array}{l}0.46 \\
0.55\end{array}$ & $\begin{array}{l}0.03 \\
0.05\end{array}$ & $\begin{array}{l}0.37 \\
0.24\end{array}$ & $\begin{array}{l}0.06 \\
0.06\end{array}$ & $\begin{array}{l}0.06 \\
0.07\end{array}$ & $\begin{array}{l}0.01 \\
0.02\end{array}$ & $\begin{array}{l}0.46 \\
0.55\end{array}$ & $\begin{array}{l}0.03 \\
0.05\end{array}$ & $\begin{array}{l}0.64 \\
0.69\end{array}$ & $\begin{array}{l}0.53 \\
0.27\end{array}$ & $\begin{array}{l}22.39 \\
27.65\end{array}$ & $\begin{array}{l}4.14 \\
3.75\end{array}$ \\
\hline $\begin{array}{l}1172 \\
1172\end{array}$ & $\begin{array}{l}\begin{array}{l}66.11 \\
56.16\end{array}\end{array}$ & $\begin{array}{l}0.24 \\
0.31\end{array}$ & $\begin{array}{l}0.06 \\
0.06\end{array}$ & $\begin{array}{l}0.07 \\
0.07\end{array}$ & $\begin{array}{l}0.02 \\
0.02\end{array}$ & $\begin{array}{l}0.55 \\
0.50\end{array}$ & $\begin{array}{l}0.05 \\
0.04\end{array}$ & $\begin{array}{l}0.24 \\
0.31\end{array}$ & $\begin{array}{l}0.06 \\
0.06\end{array}$ & $\begin{array}{l}0.07 \\
0.07\end{array}$ & $\begin{array}{l}0.02 \\
0.02\end{array}$ & $\begin{array}{l}0.55 \\
0.50\end{array}$ & $\begin{array}{l}0.05 \\
0.04\end{array}$ & $\begin{array}{l}0.69 \\
0.68\end{array}$ & $\begin{array}{l}0.27 \\
0.48\end{array}$ & $\begin{array}{l}27.65 \\
25.30\end{array}$ & $\begin{array}{l}3.75 \\
4.01\end{array}$ \\
\hline 1172 & 56.24 & 0.30 & 0.06 & 0.06 & 0.02 & 0.52 & $\begin{array}{l}0.04 \\
0.04\end{array}$ & 0.30 & 0.06 & 0.06 & 0.02 & 0.52 & 0.04 & 0.67 & $\begin{array}{l}0.40 \\
0.62\end{array}$ & 24.46 & 4.21 \\
\hline 1172 & 56.34 & 0.33 & 0.06 & 0.05 & 0.01 & 0.52 & 0.03 & 0.33 & 0.06 & 0.05 & 0.01 & 0.52 & 0.03 & 0.62 & 0.37 & 22.07 & 3.59 \\
\hline Tanzania & 33.60 & 293300 & 92750 & 153600 & 85050 & 1581500 & 229000 & 0.12 & 0.04 & 0.06 & 0.03 & 0.65 & 0.09 & 0.83 & 1.69 & 19.57 & 8.61 \\
\hline Tanzania & 39.00 & 327000 & 84000 & 105000 & 73000 & 1650000 & 324000 & 0.13 & 0.03 & 0.04 & 0.03 & 0.64 & 0.13 & 0.86 & 1.48 & 20.11 & 8.28 \\
\hline Tanzania & $\begin{array}{l}41.40 \\
446\end{array}$ & $\begin{array}{l}81000 \\
353000\end{array}$ & $\begin{array}{l}15755 \\
119000\end{array}$ & $\begin{array}{l}32300 \\
23400\end{array}$ & $\begin{array}{l}12220 \\
87700\end{array}$ & $\begin{array}{l}308000 \\
\end{array}$ & $\begin{array}{l}55750 \\
502000\end{array}$ & 0.16 & 0.03 & 0.06 & 0.02 & 0.61 & 0.11 & 0.86 & 0.98 & 23.17 & 7.05 \\
\hline Tanzania & $\begin{array}{l}44.60 \\
463\end{array}$ & $\begin{array}{l}353000 \\
2100\end{array}$ & $\begin{array}{l}119000 \\
7980\end{array}$ & $\begin{array}{l}264000 \\
13770\end{array}$ & $\begin{array}{l}87400 \\
5050\end{array}$ & 2700000 & $\begin{array}{l}502000 \\
44800\end{array}$ & 0.09 & 0.03 & 0.07 & 0.02 & 0.67 & 0.12 & 0.88 & 0.92 & $\begin{array}{l}24.22 \\
20.05\end{array}$ & $\begin{array}{l}6.49 \\
8.29\end{array}$ \\
\hline $\begin{array}{l}\text { Tanzaniaia } \\
\text { Tanzaniai }\end{array}$ & $\begin{array}{l}46.30 \\
46.60\end{array}$ & $\begin{array}{r}22100 \\
0\end{array}$ & $\begin{array}{c}7980 \\
2469809\end{array}$ & $\begin{array}{r}13700 \\
330119\end{array}$ & $\begin{array}{c}5050 \\
1311667\end{array}$ & $\begin{array}{l}182000 \\
379000\end{array}$ & $\begin{array}{c}448800 \\
10886696\end{array}$ & $\begin{array}{l}0.08 \\
0.00\end{array}$ & $\begin{array}{l}0.03 \\
0.16\end{array}$ & $\begin{array}{l}0.05 \\
0.02\end{array}$ & $\begin{array}{l}0.02 \\
0.09\end{array}$ & $\begin{array}{l}0.66 \\
0.02\end{array}$ & $\begin{array}{l}0.16 \\
0.71\end{array}$ & $\begin{array}{l}0.89 \\
0.84\end{array}$ & $\begin{array}{c}1.45 \\
13.29\end{array}$ & $\begin{array}{l}20.05 \\
15.54\end{array}$ & $\begin{array}{l}8.29 \\
8.91\end{array}$ \\
\hline Tanzania & $\begin{array}{l}\text { b } 5.00 \\
53.00\end{array}$ & 0 & $\begin{array}{r}24668009 \\
426943\end{array}$ & 324386 & 208736 & $\begin{array}{c}\text { T/99000 } \\
7960\end{array}$ & $\begin{array}{l}10886696 \\
793875\end{array}$ & $\begin{array}{l}0.00 \\
0.00\end{array}$ & $\begin{array}{l}0.16 \\
0.24\end{array}$ & $\begin{array}{l}.02 \\
0.18\end{array}$ & $\begin{array}{l}0.09 \\
0.12\end{array}$ & $\begin{array}{l}0.02 \\
0.00\end{array}$ & $\begin{array}{l}0.11 \\
0.45\end{array}$ & $\begin{array}{l}0.84 \\
0.76\end{array}$ & $\begin{array}{l}13.29 \\
16.92\end{array}$ & $\begin{array}{l}\begin{array}{l}15.54 \\
15.54\end{array} \\
1\end{array}$ & $\begin{array}{l}8.91 \\
8.91\end{array}$ \\
\hline Tanzania & 53.00 & 0 & 23665 & 32417 & 12092 & 1980 & 68293 & $\begin{array}{l}0.00 \\
0.00\end{array}$ & $\begin{array}{l}0.24 \\
0.17\end{array}$ & $\begin{array}{l}0.18 \\
0.23\end{array}$ & $\begin{array}{l}0.12 \\
0.09\end{array}$ & 0.01 & $\begin{array}{l}0.45 \\
0.49\end{array}$ & 0.83 & $\begin{array}{l}16.92 \\
11.62\end{array}$ & $\begin{array}{l}15.54 \\
15.54\end{array}$ & $\begin{array}{l}8.91 \\
8.91\end{array}$ \\
\hline Hatchitigbee $\mathrm{Fm}$. & 51.00 & 0 & 23207 & 30345 & 22276 & 407824 & 13472 & 0.00 & 0.05 & 0.06 & 0.04 & 0.82 & 0.03 & 0.74 & 1.71 & 18.72 & $\begin{array}{l}8.91 \\
8.69\end{array}$ \\
\hline Hatchitigbee $\mathrm{Fm}$. & 51.00 & 0 & 30728 & 38437 & 26255 & 470024 & 19694 & 0.00 & 0.05 & 0.07 & 0.04 & 0.80 & 0.03 & 0.73 & 170 & 18.96 & 865 \\
\hline Hatchitigbee $\mathrm{Fm}$. & 51.00 & 0 & 137457 & 173166 & 180355 & 2473728 & 103844 & 0.00 & 0.04 & 0.06 & 0.06 & 0.81 & 0.03 & 0.77 & 1.60 & 17.93 & 8.60 \\
\hline Hatchitigbee $\mathrm{Fm}$. & 51.00 & 0 & 107248 & 144800 & 91399 & 2361537 & 105902 & 0.00 & 0.04 & 0.05 & 0.03 & 0.84 & 0.04 & 0.76 & 2.21 & 16.67 & 8.86 \\
\hline Hatchitigbee $\mathrm{Fm}$. & 51.00 & 0 & 37894 & 39775 & 18491 & 676895 & 26369 & 0.00 & 0.05 & 0.05 & 0.02 & 0.85 & 0.03 & 0.69 & 1.63 & 17.61 & 8.49 \\
\hline Hatchitigbee $\mathrm{Fm}$. & 51.00 & 0 & 100784 & 140873 & 68089 & 1701328 & 80133 & 0.00 & 0.05 & 0.07 & 0.03 & 0.81 & 0.04 & 0.74 & 2.08 & 17.10 & 8.82 \\
\hline ACEX & Post-Azolla & 22000000 & 1080000 & 521000 & 182000 & 20300000 & 151000 & 0.50 & 0.02 & 0.01 & 0.00 & 0.46 & 0.00 & 0.44 & 0.10 & 5.10 & 3.38 \\
\hline ACEX & Post-Azolla & 35400000 & 1850000 & 937000 & 312000 & 32200000 & 248000 & 0.50 & 0.03 & 0.01 & 0.00 & 0.45 & 0.00 & 0.45 & 0.08 & 5.83 & 3.38 \\
\hline ACEX & Post-Azolla & 19800000 & 946000 & 477000 & 161000 & 17800000 & 131000 & 0.50 & 0.02 & 0.01 & 0.00 & 0.45 & 0.00 & 0.45 & 0.10 & 4.83 & 3.38 \\
\hline ACEX & Post-Azolla & 28400000 & 1540000 & 740000 & 249000 & 26300000 & 204000 & 0.49 & 0.03 & 0.01 & 0.00 & 0.46 & 0.00 & 0.44 & 0.08 & 6.24 & 3.38 \\
\hline ACEX & Post-Azolla & 30100000 & 1560000 & 773000 & 237000 & 26500000 & 201000 & 0.51 & 0.03 & 0.01 & 0.00 & 0.45 & 0.00 & 0.44 & 0.08 & 5.70 & 3.38 \\
\hline $\begin{array}{l}\text { ACEX } \\
A C F X\end{array}$ & Azolla & $\begin{array}{l}3670000 \\
36100000\end{array}$ & $\begin{array}{l}191000 \\
\end{array}$ & $\begin{array}{l}99600 \\
\\
1144000\end{array}$ & $\begin{array}{l}29900 \\
35000\end{array}$ & $\begin{array}{l}3470000 \\
3780000\end{array}$ & $\begin{array}{l}33500 \\
33500\end{array}$ & 0.49 & 0.03 & 0.01 & 0.00 & 0.46 & 0.00 & 0.46 & 0.06 & 6.02 & $\begin{array}{l}3.39 \\
3.40\end{array}$ \\
\hline $\begin{array}{l}\text { ACEX } \\
\text { ACEX }\end{array}$ & $\begin{array}{l}\text { Azolla } \\
\text { Azolla }\end{array}$ & $\begin{array}{l}3661000000 \\
4840000\end{array}$ & $\begin{array}{l}21300000 \\
280000\end{array}$ & $\begin{array}{l}11440000 \\
1161000\end{array}$ & $\begin{array}{l}3500000 \\
45000\end{array}$ & $\begin{array}{l}378000000 \\
5010000\end{array}$ & $\begin{array}{l}335000 \\
46300\end{array}$ & $\begin{array}{l}0.46 \\
0.47\end{array}$ & $\begin{array}{l}0.03 \\
0.03\end{array}$ & $\begin{array}{l}0.01 \\
0.02\end{array}$ & $\begin{array}{l}0.00 \\
0.00\end{array}$ & $\begin{array}{l}0.49 \\
0.48\end{array}$ & $\begin{array}{l}0.00 \\
0.00\end{array}$ & $\begin{array}{l}0.46 \\
0.47\end{array}$ & $\begin{array}{l}0.15 \\
0.16\end{array}$ & $\begin{array}{l}8.73 \\
8.65\end{array}$ & $\begin{array}{l}3.40 \\
3.41\end{array}$ \\
\hline ACEX & $\begin{array}{l}\text { Azond } \\
\text { Azolla }\end{array}$ & 8720000 & 515000 & 301000 & 85900 & 9600000 & 80200 & 0.45 & 0.03 & $\begin{array}{l}0.02 \\
0.02\end{array}$ & 0.00 & $\begin{array}{l}0.48 \\
0.50\end{array}$ & $\begin{array}{l}0.00 \\
0.00\end{array}$ & $\begin{array}{l}0.41 \\
0.48\end{array}$ & $\begin{array}{l}0.16 \\
0.15\end{array}$ & $\begin{array}{l}8.65 \\
9.83\end{array}$ & $\begin{array}{l}3.41 \\
3.42\end{array}$ \\
\hline ACEX & Azolla & 4030000 & 297000 & 154000 & 40600 & 4470000 & 41900 & 0.45 & 0.03 & 0.02 & 0.00 & 0.49 & 0.00 & 0.44 & 0.19 & $\begin{array}{l}3.03 \\
12.71\end{array}$ & $\begin{array}{l}.42 \\
3.40\end{array}$ \\
\hline ACEX & Azolla & 3390000 & 199000 & 112000 & 31400 & 3470000 & 26900 & 0.47 & 0.03 & 0.02 & 0.00 & 0.48 & 0.00 & 0.46 & 0.15 & 8.77 & 3.40 \\
\hline ACEX & Azolla & 21800000 & 1470000 & 888000 & 249000 & 24900000 & 235000 & 0.44 & 0.03 & 0.02 & 0.01 & 0.50 & 0.00 & 0.48 & 0.13 & 11.88 & 3.44 \\
\hline ACEX & Azolla & 11700000 & 866000 & 537000 & 139000 & 14700000 & 148000 & 0.42 & 0.03 & 0.02 & 0.00 & 0.52 & 0.01 & 0.49 & 0.21 & 14.43 & 3.46 \\
\hline $\begin{array}{l}\text { ACEX } \\
\text { ACEX }\end{array}$ & Azolla & 17300000 & $\begin{array}{l}1310000 \\
1414000\end{array}$ & $\begin{array}{l}796000 \\
8400\end{array}$ & $\begin{array}{l}221000 \\
23800\end{array}$ & $\begin{array}{l}22400000 \\
245000\end{array}$ & $\begin{array}{l}221000 \\
255000\end{array}$ & 0.41 & 0.03 & 0.02 & 0.01 & 0.53 & 0.01 & 0.49 & 0.24 & 14.57 & 3.47 \\
\hline $\begin{array}{l}\text { ACEX } \\
\text { ACEX }\end{array}$ & $\begin{array}{l}\text { Azolla } \\
\text { Aarllla }\end{array}$ & $\begin{array}{l}21400000 \\
4500000\end{array}$ & $\begin{array}{l}1410000 \\
255000\end{array}$ & $\begin{array}{l}841000 \\
140000\end{array}$ & $\begin{array}{l}238000 \\
3800\end{array}$ & $\begin{array}{r}24500000 \\
4570000\end{array}$ & $\begin{array}{l}252000 \\
46400\end{array}$ & $\begin{array}{l}0.44 \\
0.47\end{array}$ & 0.03 & 0.02 & $\begin{array}{l}0.00 \\
0.00\end{array}$ & $\begin{array}{l}0.50 \\
0.48\end{array}$ & 0.01 & $\begin{array}{l}0.49 \\
0.48\end{array}$ & 0.12 & $\begin{array}{l}11.58 \\
7.59\end{array}$ & $\begin{array}{l}3.43 \\
3.40\end{array}$ \\
\hline $\begin{array}{l}\text { ACEX } \\
\text { ACEX }\end{array}$ & $\begin{array}{l}\text { Azolaa } \\
\text { Azolla }\end{array}$ & $\begin{array}{l}45000000 \\
4680000\end{array}$ & $\begin{array}{l}265000 \\
263000\end{array}$ & $\begin{array}{l}1400000 \\
128000\end{array}$ & $\begin{array}{l}38800 \\
35100\end{array}$ & $\begin{array}{l}45700000 \\
4120000\end{array}$ & $\begin{array}{l}464000 \\
40300\end{array}$ & $\begin{array}{l}0.47 \\
0.51\end{array}$ & $\begin{array}{l}0.03 \\
0.03\end{array}$ & $\begin{array}{l}0.01 \\
0.01\end{array}$ & $\begin{array}{l}0.00 \\
0.00\end{array}$ & $\begin{array}{l}0.48 \\
0.44\end{array}$ & $\begin{array}{l}0.00 \\
0.00\end{array}$ & $\begin{array}{l}0.48 \\
0.44\end{array}$ & $\begin{array}{l}0.16 \\
0.12\end{array}$ & $\begin{array}{l}7.59 \\
6.36\end{array}$ & $\begin{array}{l}3.40 \\
3.38\end{array}$ \\
\hline $\begin{array}{l}\text { ACEX } \\
\text { ACEX }\end{array}$ & $\begin{array}{l}\text { Azolaa } \\
\text { Azolla }\end{array}$ & $\begin{array}{l}46880000 \\
3580000\end{array}$ & $\begin{array}{l}2230000 \\
177000\end{array}$ & $\begin{array}{l}1780000 \\
89900\end{array}$ & $\begin{array}{l}35100 \\
26900\end{array}$ & $\begin{array}{l}41200000 \\
3080000\end{array}$ & $\begin{array}{l}\begin{array}{l}403000 \\
32100\end{array}\end{array}$ & $\begin{array}{l}0.51 \\
0.51\end{array}$ & $\begin{array}{l}0.03 \\
0.03\end{array}$ & $\begin{array}{l}.01 \\
0.01\end{array}$ & $\begin{array}{l}0.00 \\
0.00\end{array}$ & $\begin{array}{l}0.44 \\
0.44\end{array}$ & $\begin{array}{l}0.00 \\
0.00\end{array}$ & $\begin{array}{l}0.44 \\
0.46\end{array}$ & $\begin{array}{l}.1 .12 \\
0.13\end{array}$ & $\begin{array}{l}6.36 \\
4.93\end{array}$ & $\begin{array}{l}3.38 \\
3.39\end{array}$ \\
\hline ACEX & Azolla & 57400000 & 2910000 & $\begin{array}{l}89500 \\
1280000\end{array}$ & $\begin{array}{l}265000 \\
454000\end{array}$ & 497000000 & 455000 & 0.51 & 0.03 & 0.01 & 0.00 & $\begin{array}{l}0.44 \\
0.44\end{array}$ & 0.00 & $\begin{array}{l}0.40 \\
0.43\end{array}$ & $\begin{array}{l}.113 \\
0.11\end{array}$ & $\begin{array}{l}4.99 \\
4.99\end{array}$ & $\begin{array}{l}3.39 \\
3.37\end{array}$ \\
\hline ACEX & Azolla & 4950000 & 257000 & 109000 & 34100 & 4250000 & 33500 & 0.51 & 0.0 & 0.01 & 0.00 & 0.44 & 0.0 & 0.44 & 0.10 & 506 & 3.37 \\
\hline ACEX & Azolla & 25600000 & 1260000 & 575000 & 205000 & 23600000 & 187000 & 0.5 & 0.0 & 0.01 & 0 & 0.46 & 0.0 & 0.43 & 0.1 & $\begin{array}{ll}4.099 \\
4.99\end{array}$ & 3.37 \\
\hline ACEX & Azolla & 59000000 & 2780000 & 1320000 & 4740 & 50000000 & 413000 & 0.52 & 0.02 & 0.01 & 0. & 0.44 & 0.00 & 0.38 & 0. & -0.42 & 3.91 \\
\hline ACEX & Azolla & 39400000 & 1920000 & 908000 & 302000 & 34000000 & 289000 & 0.51 & 0.02 & 0.01 & 0.00 & 0.44 & 0.00 & 0.44 & 0.12 & 4.79 & 3.38 \\
\hline ACEX & Azolla & 21200000 & 977000 & 470000 & 157000 & 19000000 & 148000 & 0.51 & 0.02 & 0.01 & 0.00 & 0.45 & 0.00 & 0.44 & 0.12 & 4.31 & 3.38 \\
\hline ACEX & Azolla & 3340000 & 170000 & 93800 & 28900 & 2710000 & 25900 & 0.52 & 0.03 & 0.01 & 0.00 & 0.43 & 0.00 & 0.47 & 0.10 & 5.86 & 3.41 \\
\hline ACEX & Azolla & 49100000 & 2550000 & 1260000 & 406000 & 44200000 & 418000 & 0.50 & 0.03 & 0.01 & 0.00 & 0.45 & 0.00 & 0.45 & 0.05 & 5.58 & 3.38 \\
\hline ACEX & Azolla & 46900000 & 2460000 & 1140000 & 375000 & 41500000 & 361000 & 0.51 & 0.03 & 0.01 & 0.00 & 0.45 & 0.00 & 0.43 & 0.06 & 5.56 & 3.38 \\
\hline ACEX & Azolla & 31100 & 1670000 & 775000 & 254000 & 29300000 & 259000 & 0.49 & 0.03 & 0.01 & 0.00 & 0.46 & 0.00 & 0.44 & 0.05 & 6.12 & 3.38 \\
\hline ACEX & Azolla & 25400000 & 1200000 & 556000 & 188000 & 22800000 & 195000 & 0.50 & 0.02 & 0.01 & 0.00 & 0.45 & 0.00 & 0.44 & 0.11 & 4.43 & 3.38 \\
\hline $\begin{array}{l}\text { ACEX } \\
\text { ACEX }\end{array}$ & Azolla & $\begin{array}{l}5890000 \\
07000\end{array}$ & $\begin{array}{l}305000 \\
198000\end{array}$ & $\begin{array}{l}123000 \\
80800\end{array}$ & $\begin{array}{l}41500 \\
28500\end{array}$ & $\begin{array}{l}5030000 \\
340000\end{array}$ & $\begin{array}{l}44300 \\
27100\end{array}$ & $\begin{array}{l}0.52 \\
0.52\end{array}$ & 0.03 & 0.01 & & 0.44 & 0.00 & 0.41 & 0.08 & 4.88 & 37 \\
\hline $\begin{array}{l}\text { ACEX } \\
\text { ACEX }\end{array}$ & Azolla & $\begin{array}{l}4070000 \\
33200\end{array}$ & $\begin{array}{l}198000 \\
15000\end{array}$ & $\begin{array}{l}80800 \\
67100\end{array}$ & $\begin{array}{l}28500 \\
21400\end{array}$ & $\begin{array}{l}3400000 \\
26000\end{array}$ & $\begin{array}{l}27100 \\
23000\end{array}$ & $\begin{array}{l}0.52 \\
0.53\end{array}$ & 0.03 & 0.01 & 0.00 & 0.44 & 0.00 & 0.41 & 0.07 & 4.42 & 3.37 \\
\hline $\begin{array}{l}\text { ACEX } \\
\text { ACEX }\end{array}$ & $\begin{array}{l}\text { Azolla } \\
\text { Azolla }\end{array}$ & $\begin{array}{l}33200000 \\
8760000\end{array}$ & $\begin{array}{l}145000 \\
436000\end{array}$ & $\begin{array}{r}67100 \\
211000\end{array}$ & $\begin{array}{l}21100 \\
62800\end{array}$ & $\begin{array}{l}26600000 \\
7610000\end{array}$ & $\begin{array}{l}23000 \\
74200\end{array}$ & $\begin{array}{l}0.53 \\
0.51\end{array}$ & $\begin{array}{l}0.02 \\
0.03\end{array}$ & $\begin{array}{l}0.01 \\
0.01\end{array}$ & $\begin{array}{l}0.00 \\
0.00\end{array}$ & $\begin{array}{l}0.43 \\
0.44\end{array}$ & $\begin{array}{l}0.00 \\
0.00\end{array}$ & $\begin{array}{l}0.43 \\
0.44\end{array}$ & $\begin{array}{l}0.09 \\
0.13\end{array}$ & $\begin{array}{l}3.64 \\
4.88\end{array}$ & $\begin{array}{l}3.38 \\
3.38\end{array}$ \\
\hline ACEX & $\begin{array}{l}\text { Azonda } \\
\text { Azolla }\end{array}$ & 4080000 & $\begin{array}{l}4000000 \\
20000\end{array}$ & 87900 & 27200 & 3460000 & $\begin{array}{l}47200 \\
37000\end{array}$ & $\begin{array}{l}0.51 \\
0.52\end{array}$ & $\begin{array}{l}0.03 \\
0.03\end{array}$ & $\begin{array}{l}.01 \\
0.01\end{array}$ & $\begin{array}{l}0.00 \\
0.00\end{array}$ & $\begin{array}{l}0.44 \\
0.44\end{array}$ & $\begin{array}{l}0.00 \\
0.00\end{array}$ & $\begin{array}{l}0.44 \\
0.43\end{array}$ & $\begin{array}{l}0.13 \\
0.09\end{array}$ & $\begin{array}{l}4.88 \\
4.26\end{array}$ & $\begin{array}{l}3.38 \\
3.38\end{array}$ \\
\hline ACEX & Azolla & 8960000 & 383000 & 187000 & 64300 & 7070000 & 61200 & 0.54 & 0.02 & 0.01 & 0.00 & 0.42 & 0.00 & 0.45 & 0.13 & 3.93 & 3.39 \\
\hline$A C$ & Azolla & 5150000 & 254000 & 115000 & 34600 & 4300000 & 39 & & 0.03 & 0.01 & 0.00 & 0.43 & 0 & & & 4.55 & 38 \\
\hline ACEX & Azolla & 4770000 & 217000 & 110000 & 33400 & 4200000 & 3910 & 0. & 0.02 & 0.01 & & 0.45 & 0 & 0. & & 4.09 & 39 \\
\hline ACEX & Azolla & 8030000 & 434000 & 193000 & 56700 & 7020000 & 67000 & $e^{2}+2$ & 0.03 & 0.01 & & 0.44 & 0.00 & 0 & 15 & 5.53 & 38 \\
\hline ACEX & Azolla & 5480000 & 245000 & 122000 & 35700 & 4540000 & 41200 & 0.52 & 0.02 & 0.01 & 0.00 & 0.43 & 0.00 & 0.45 & 12 & 3.83 & 39 \\
\hline ACEX & Azolla & 4040000 & 180000 & 78000 & 25300 & 3310000 & 30100 & 0.53 & 0.02 & 0.01 & 0.00 & 0.43 & 0.00 & 0.43 & 0.08 & 3.47 & 3.38 \\
\hline ACEX & Pre-Azolla/post-ETM2 & 2620000 & 124000 & 63400 & 23300 & 2290000 & 23000 & 0.51 & 0.02 & 0.01 & 0.00 & 0.45 & 0.00 & 0.47 & 0.13 & 4.35 & 3.38 \\
\hline
\end{tabular}


Eocene dataset for OPTIMAL

\begin{tabular}{|c|c|c|c|c|c|c|c|c|c|c|c|c|c|c|c|c|c|}
\hline SITE & AGE & $\begin{array}{c}1302 \\
\text { GDGTO } \\
\end{array}$ & $\begin{array}{l}1300 \\
\text { GDGT1 }\end{array}$ & $\begin{array}{r}1298 \\
\text { GDGT2 } \\
\end{array}$ & $\begin{array}{r}1296 \\
\text { GDGT3 } \\
\end{array}$ & $\begin{array}{l}1292 \\
\text { CREN } \\
\end{array}$ & $\begin{array}{l}1292^{\prime} \\
\text { CREN }\end{array}$ & Fr. 1302 & Fr. 1300 & Fr.1298 & Fr. 1296 & Fr.1292 & Fr.1292 & $\begin{array}{c}\text { TEX } \\
\text { (published) }\end{array}$ & D_nearest & $\begin{array}{c}\text { OPTTMAL } \\
\text { SST }\end{array}$ & $\begin{array}{c}\text { OPTIMAL } \\
\text { StDev } \\
\end{array}$ \\
\hline ACEX & 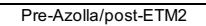 & 944000 & 57500 & 27300 & 10100 & 835000 & 9920 & 0.50 & 0.03 & 0.01 & 0.01 & 0.44 & 0.01 & 0.45 & 0.15 & 6.59 & 3.38 \\
\hline ACEX & Pre-Azolla/post-ETM2 & 2310000 & 125000 & 65700 & 22600 & 2100000 & 23100 & 0.50 & 0.03 & 0.01 & 0.00 & 0.45 & 0.00 & 0.47 & 0.12 & 5.76 & 3.38 \\
\hline ACEX & Pre-Azolla/post-ETM2 & 817000 & 48900 & 23700 & 9560 & 704000 & 9280 & 0.51 & 0.03 & 0.01 & 0.01 & 0.44 & 0.01 & 0.47 & 0.25 & 5.74 & 3.40 \\
\hline ACEX & Pre-Azolla/post-ETM2 & 1830000 & 99200 & 45600 & 16700 & 1500000 & 11900 & 0.52 & 0.03 & 0.01 & 0.00 & 0.43 & 0.00 & 0.43 & 0.08 & 6.19 & 3.39 \\
\hline ACEX & Pre-Azolla/post-ETM2 & 4320000 & 203000 & 119000 & 47800 & 3500000 & 37300 & 0.53 & 0.02 & 0.01 & 0.01 & 0.43 & 0.00 & 0.50 & 0.15 & 4.02 & 3.46 \\
\hline ACEX & Pre-Azolla/post-ETM2 & 2190000 & 87100 & 49000 & 22400 & 1560000 & 14700 & 0.56 & 0.02 & 0.01 & 0.01 & 0.40 & 0.00 & 0.50 & 0.18 & 4.82 & 3.60 \\
\hline ACEX & ETM2 & 4010000 & 492000 & 403000 & 92300 & 3620000 & 125000 & 0.46 & 0.06 & 0.05 & 0.01 & 0.41 & 0.01 & 0.56 & 0.38 & 13.18 & 4.10 \\
\hline ACEX & ETM2 & 3420000 & 398000 & 315000 & 78700 & 27700000 & 94000 & 0.48 & 0.06 & 0.04 & 0.01 & 0.39 & 0.01 & 0.55 & 0.58 & 10.77 & 4.36 \\
\hline ACEX & ETM2 & 1400000 & 177000 & 156000 & 33300 & 1220000 & 46800 & 0.46 & 0.06 & 0.05 & 0.01 & 0.40 & 0.02 & 0.57 & 0.52 & 13.94 & 4.41 \\
\hline ACEX & ЕTM2 & 6980000 & 1010000 & 908000 & 181000 & 6770000 & 285000 & 0.43 & 0.06 & 0.06 & 0.01 & 0.42 & 0.02 & 0.58 & 0.43 & 16.70 & 4.22 \\
\hline ACEX & ЕTM2 & 2880000 & 393000 & 352000 & 72400 & 2570000 & 120000 & 0.45 & 0.06 & 0.06 & 0.01 & 0.40 & 0.02 & 0.58 & 0.58 & 15.13 & 4.48 \\
\hline ACEX & ETM2 & 1550000 & 211000 & 192000 & 40400 & 1350000 & 71100 & 0.45 & 0.06 & 0.06 & 0.01 & 0.40 & 0.02 & 0.59 & 0.65 & 14.62 & 4.73 \\
\hline ACEX & ETM2 & 739000 & 82100 & 71500 & 17400 & 707000 & 23200 & 0.45 & 0.05 & 0.04 & 0.01 & 0.43 & 0.01 & 0.58 & 0.43 & 12.83 & 4.13 \\
\hline ACEX & ETM2 & 3250000 & 295000 & 201000 & 63100 & 3510000 & 83600 & 0.44 & 0.04 & 0.03 & 0.01 & 0.47 & 0.01 & 0.54 & 0.40 & 11.11 & 3.65 \\
\hline ACEX & ETM2 & 1190000 & 178000 & 155000 & 30300 & $\begin{array}{r}961000 \\
557500\end{array}$ & 51700 & 0.46 & 0.07 & 0.06 & 0.01 & 0.37 & 0.02 & 0.57 & 0.71 & $\begin{array}{l}15.46 \\
20.20\end{array}$ & 4.90 \\
\hline $\begin{array}{l}\text { ACEX } \\
\text { ACEX }\end{array}$ & $\begin{array}{l}\text { ETM2 } \\
\text { ETMM }\end{array}$ & $\begin{array}{l}60000000 \\
55210000\end{array}$ & $\begin{array}{l}10700000 \\
839000\end{array}$ & $\begin{array}{l}1080000 \\
85100\end{array}$ & $\begin{array}{l}220000 \\
13600\end{array}$ & $\begin{array}{r}5570000 \\
531000\end{array}$ & $\begin{array}{r}353000 \\
26300\end{array}$ & 0.42 & 0.07 & 0.08 & 0.02 & 0.39 & 0.02 & 0.61 & 0.83 & 20.25 & 5.75 \\
\hline $\begin{array}{l}\text { ACEX } \\
\text { ACEX }\end{array}$ & $\begin{array}{l}\text { ETT2 } \\
\text { ETM2 }\end{array}$ & $\begin{array}{l}55100000 \\
8180000\end{array}$ & $\begin{array}{l}8890000 \\
1470000\end{array}$ & $\begin{array}{l}851000 \\
1500000\end{array}$ & $\begin{array}{l}136000 \\
219000\end{array}$ & $\begin{array}{l}53110000 \\
891000\end{array}$ & $\begin{array}{l}263000 \\
48200\end{array}$ & $\begin{array}{l}0.41 \\
0.39\end{array}$ & $\begin{array}{l}0.07 \\
0.07\end{array}$ & $\begin{array}{l}0.07 \\
0.07\end{array}$ & $\begin{array}{l}0.01 \\
0.01\end{array}$ & $\begin{array}{l}0.42 \\
0.43\end{array}$ & $\begin{array}{l}0.02 \\
0.02\end{array}$ & $\begin{array}{l}0.60 \\
0.60\end{array}$ & $\begin{array}{l}0.51 \\
0.51\end{array}$ & $\begin{array}{l}20.59 \\
\end{array}$ & $\begin{array}{l}4.28 \\
4.08\end{array}$ \\
\hline $\begin{array}{l}\text { ACEX } \\
\text { ACEX }\end{array}$ & $\begin{array}{l}\text { ETM2 } \\
\text { ETM2 }\end{array}$ & $\begin{array}{l}1880000 \\
765000\end{array}$ & $\begin{array}{l}\begin{array}{l}14700000 \\
154000\end{array} \\
\end{array}$ & $\begin{array}{l}15000000 \\
206000\end{array}$ & $\begin{array}{l}219000 \\
32700\end{array}$ & $\begin{array}{l}89100000 \\
1030000\end{array}$ & $\begin{array}{r}482000 \\
83100\end{array}$ & $\begin{array}{l}0.39 \\
0.34\end{array}$ & $\begin{array}{l}0.07 \\
0.07\end{array}$ & $\begin{array}{l}0.07 \\
0.09\end{array}$ & $\begin{array}{l}0.01 \\
0.01\end{array}$ & $\begin{array}{l}0.43 \\
0.45\end{array}$ & $\begin{array}{l}0.02 \\
0.04\end{array}$ & $\begin{array}{l}0.60 \\
0.68\end{array}$ & $\begin{array}{l}0.51 \\
0.57\end{array}$ & $\begin{array}{l}22.19 \\
27.57\end{array}$ & $\begin{array}{l}4.05 \\
4.33\end{array}$ \\
\hline $\begin{array}{l}\text { ALEX } \\
\text { ACEX }\end{array}$ & $\begin{array}{l}\text { ETM2 } \\
\text { ETM2 }\end{array}$ & 72700000 & $\begin{array}{l}15400000 \\
152000\end{array}$ & 2070000 & 258000 & $\begin{array}{l}10300000 \\
9900000\end{array}$ & 661000 & $\begin{array}{l}0.34 \\
0.34\end{array}$ & 0.07 & $\begin{array}{l}0.109 \\
0.10\end{array}$ & 0.01 & $\begin{array}{l}0.45 \\
0.46\end{array}$ & $\begin{array}{l}0.04 \\
0.03\end{array}$ & $\begin{array}{l}0.08 \\
0.66\end{array}$ & 0.51 & 26.45 & $\begin{array}{l}4.33 \\
4.26\end{array}$ \\
\hline ACEX & ETM2 & 4430000 & 667000 & 534000 & 128000 & 5260000 & 201000 & 0.39 & 0.06 & 0.05 & 0.01 & 0.47 & 0.02 & 0.56 & 0.31 & 18.35 & $\begin{array}{l}3.66 \\
3.66\end{array}$ \\
\hline ACEX & ETM2 & 6360000 & 697000 & 478000 & 185000 & 6670000 & 148000 & 0.44 & 0.05 & 0.03 & 0.01 & 0.46 & 0.01 & 0.54 & 0.35 & 10.85 & 3.94 \\
\hline ACEX & ETM2 & 3420000 & 369000 & 235000 & 87800 & 3530000 & 77300 & 0.44 & 0.05 & 0.03 & 0.01 & 0.46 & 0.01 & 0.52 & 0.20 & 10.11 & 3.73 \\
\hline $\begin{array}{l}\text { ACEX } \\
\end{array}$ & $\begin{array}{l}\text { ETM2 } \\
\end{array}$ & $\begin{array}{r}4020000 \\
40700\end{array}$ & $\begin{array}{l}547000 \\
76700\end{array}$ & $\begin{array}{l}428000 \\
99300\end{array}$ & 141000 & $\begin{array}{l}4540000 \\
6150\end{array}$ & 129000 & 0.41 & 0.06 & 0.04 & 0.01 & 0.46 & 0.01 & 0.56 & 0.47 & 15.94 & 4.18 \\
\hline $\begin{array}{l}\text { ACEX } \\
\text { ACEX }\end{array}$ & $\begin{array}{l}\text { ETM2 } \\
\text { ETMM }\end{array}$ & $\begin{array}{r}40700000 \\
479000\end{array}$ & $\begin{array}{c}7670000 \\
97400\end{array}$ & $\begin{array}{l}993000 \\
127000\end{array}$ & $\begin{array}{l}140000 \\
17500\end{array}$ & $\begin{array}{l}6150000 \\
655000\end{array}$ & $\begin{array}{l}4060000 \\
50400\end{array}$ & $\begin{array}{l}0.32 \\
0.33\end{array}$ & $\begin{array}{l}0.06 \\
0.07\end{array}$ & $\begin{array}{l}0.08 \\
0.09\end{array}$ & $\begin{array}{l}0.01 \\
0.01\end{array}$ & $\begin{array}{l}0.49 \\
0.47\end{array}$ & $\begin{array}{l}0.03 \\
0.04\end{array}$ & $\begin{array}{l}0.67 \\
0.67\end{array}$ & $\begin{array}{l}0.47 \\
0.50\end{array}$ & $\begin{array}{l}22.90 \\
22.02\end{array}$ & $\begin{array}{l}3.80 \\
4.01\end{array}$ \\
\hline $\begin{array}{l}\text { ACEX } \\
\text { ACEX }\end{array}$ & $\begin{array}{l}\text { TTM2 } \\
\text { ETM2 }\end{array}$ & $\begin{array}{l}4690000 \\
3060000\end{array}$ & $\begin{array}{l}94400 \\
736000\end{array}$ & $\begin{array}{l}127000 \\
862000\end{array}$ & $\begin{array}{l}17500 \\
92100\end{array}$ & $\begin{array}{r}6650000 \\
4120000\end{array}$ & $\begin{array}{r}50400 \\
286000\end{array}$ & $\begin{array}{l}0.33 \\
0.33\end{array}$ & $\begin{array}{l}0.07 \\
0.08\end{array}$ & $\begin{array}{l}0.099 \\
0.09\end{array}$ & $\begin{array}{l}0.01 \\
0.01\end{array}$ & $\begin{array}{l}0.47 \\
0.45\end{array}$ & $\begin{array}{l}0.04 \\
0.03\end{array}$ & $\begin{array}{l}0.67 \\
0.63\end{array}$ & $\begin{array}{l}0.50 \\
0.25\end{array}$ & $\begin{array}{l}26.02 \\
26.80\end{array}$ & $\begin{array}{l}4.01 \\
3.92\end{array}$ \\
\hline $\begin{array}{l}\text { ACEX } \\
\text { ACEX }\end{array}$ & $\begin{array}{l}\text { ETM2 } \\
\text { ETM2 }\end{array}$ & 4590000 & 790000 & 834000 & 111000 & $\begin{array}{l}\begin{array}{r}1220000 \\
540000\end{array}\end{array}$ & 271000 & 0.38 & $\begin{array}{l}0.08 \\
0.07\end{array}$ & $\begin{array}{l}0.09 \\
0.07\end{array}$ & $\begin{array}{l}0.01 \\
0.01\end{array}$ & $\begin{array}{l}0.45 \\
0.45\end{array}$ & $\begin{array}{l}0.03 \\
0.02\end{array}$ & $\begin{array}{l}0.03 \\
0.61\end{array}$ & $\begin{array}{l}0.25 \\
0.29\end{array}$ & $\begin{array}{l}21.70 \\
21.76\end{array}$ & $\begin{array}{l}3.92 \\
3.85\end{array}$ \\
\hline ACEX & ETM2 & 7170000 & 1250000 & 1280000 & 169000 & 7930000 & 356000 & 0.39 & 0.07 & 0.07 & 0.01 & 0.44 & 0.02 & 0.59 & 0.36 & 21.90 & $\begin{array}{l}3.00 \\
3.95\end{array}$ \\
\hline ACEX & ETM2 & 1560000 & 253000 & 240000 & 27200 & 1430000 & 76500 & 0.43 & 0.07 & 0.07 & 0.01 & 0.40 & 0.02 & 0.58 & 0.44 & 22.29 & 3.97 \\
\hline ACEX & ietween PETM and ETM & 695000 & 104000 & 77900 & 11700 & 558000 & 25800 & 0.47 & 0.07 & 0.05 & 0.01 & 0.38 & & 0.53 & 0.22 & 18.87 & 3.58 \\
\hline ACEX & ietween PETM and ETM & 2800000 & 487000 & 485000 & 51300 & 3280000 & 195000 & 0.38 & 0.07 & 0.07 & 0.01 & 0.45 & 0. & 0.60 & 0.34 & 22.03 & 3.71 \\
\hline ACEX & ietween PETM and ETM & 6350000 & 1070000 & 1020000 & 99800 & 6860000 & 321000 & 0.40 & 0.07 & 0.06 & 0.01 & 0.44 & 0.02 & 0.57 & 0.43 & 21.24 & 3.79 \\
\hline ACEX & ietween PETM and ETM & 4800000 & 685000 & 567000 & 75200 & 4340000 & 192000 & 0.45 & 0.06 & 0.05 & 0.01 & 0.41 & $0.0-0>>3$ & 0.55 & 0.31 & 19.01 & 3.68 \\
\hline ACEX & ietween PETM and ETM & 4870000 & 796000 & 781000 & 74400 & 5200000 & 238000 & 0.41 & 0.07 & 0.07 & 0.01 & 0.43 & 0.02 & 0.58 & 0.46 & 21.37 & 3.88 \\
\hline ACEX & ietween PETM and ETM & 1340000 & 244000 & 278000 & 28400 & 1460000 & 86700 & 0.39 & 0.07 & 0.08 & 0.01 & 0.42 & 0.03 & 0.62 & 0.54 & 24.20 & 4.26 \\
\hline ACEX & ietween PETM and ETM & 6470000 & 935000 & 862000 & 87200 & 6960000 & 260000 & 0.42 & 0.06 & 0.06 & 0.01 & 0.45 & 0.02 & 0.56 & 0.36 & 19.74 & 3.73 \\
\hline ACEX & ;etween PETM and ETM & 1430000 & 242000 & 241000 & 28500 & 1340000 & 71600 & 0.43 & 0.07 & 0.07 & 0.01 & 0.40 & 0.02 & 0.58 & 0.46 & 23.00 & 4.14 \\
\hline ACEX & ietween PETM and ETM & 14100000 & 2190000 & 2130000 & 230000 & 14200000 & 641000 & 0.42 & 0.07 & 0.06 & 0.01 & 0.42 & 0.02 & 0.58 & 0.45 & 21.45 & 3.92 \\
\hline $\begin{array}{l}\text { ACEX } \\
\text { ACEX }\end{array}$ & etween PETM and ETM & $\begin{array}{l}7140000 \\
2070000\end{array}$ & $\begin{array}{l}1170000 \\
333000\end{array}$ & $\begin{array}{l}11110000 \\
28700\end{array}$ & $\begin{array}{l}116000 \\
38400\end{array}$ & $\begin{array}{r}6880000 \\
2420000\end{array}$ & $\begin{array}{l}316000 \\
101000\end{array}$ & 0.43 & 0.07 & 0.07 & 0.01 & 0.41 & 0.02 & 0.57 & 0.52 & 21.71 & 3.93 \\
\hline $\begin{array}{l}\text { ACEX } \\
\text { ACEX }\end{array}$ & 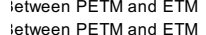 & $\begin{array}{l}2070000 \\
5960000\end{array}$ & $\begin{array}{l}313000 \\
998000\end{array}$ & $\begin{array}{r}287000 \\
1110000\end{array}$ & $\begin{array}{r}38400 \\
116000\end{array}$ & $\begin{aligned} 24200000 \\
6290000\end{aligned}$ & $\begin{array}{l}101000 \\
324000\end{array}$ & $\begin{array}{l}0.40 \\
0.40\end{array}$ & 0.06 & 0.05 & 0.01 & 0.46 & 0.02 & 0.58 & 0.36 & 20.22 & 3.64 \\
\hline $\begin{array}{l}\text { ACEX } \\
\text { ACEX }\end{array}$ & $\begin{array}{l}\text { ietween PETM and ETM } \\
\text { etween PETM and ETM }\end{array}$ & $\begin{array}{r}59600000 \\
900000\end{array}$ & $\begin{array}{l}998000 \\
115000\end{array}$ & $\begin{array}{c}111100000 \\
77300\end{array}$ & $\begin{array}{l}1160000 \\
16600\end{array}$ & $\begin{array}{l}62900000 \\
780000\end{array}$ & $\begin{array}{l}324000 \\
27800\end{array}$ & $\begin{array}{l}0.40 \\
0.47\end{array}$ & $\begin{array}{l}0.07 \\
0.06\end{array}$ & $\begin{array}{l}0.08 \\
0.04\end{array}$ & $\begin{array}{l}0.01 \\
0.01\end{array}$ & $\begin{array}{l}0.43 \\
0.41\end{array}$ & $\begin{array}{l}0.02 \\
0.01\end{array}$ & $\begin{array}{l}0.61 \\
0.51\end{array}$ & $\begin{array}{l}0.52 \\
0.38\end{array}$ & $\begin{array}{l}23.34 \\
13.87\end{array}$ & $\begin{array}{l}4.24 \\
3.62\end{array}$ \\
\hline $\begin{array}{l}\text { ACEX } \\
\text { ACE }\end{array}$ & etween PETM and ETM & 9400000 & $\begin{array}{l}115000 \\
1360000\end{array}$ & $\begin{array}{l}77300 \\
1230000\end{array}$ & $\begin{array}{l}16600 \\
155000\end{array}$ & $\begin{array}{l}7800000 \\
9160000\end{array}$ & $\begin{array}{l}27800 \\
357000\end{array}$ & $\begin{array}{l}0.47 \\
0.43\end{array}$ & $\begin{array}{l}0.06 \\
0.06\end{array}$ & $\begin{array}{l}0.04 \\
0.06\end{array}$ & $\begin{array}{l}0.01 \\
0.01\end{array}$ & $\begin{array}{l}0.41 \\
0.42\end{array}$ & $\begin{array}{l}0.01 \\
0.02\end{array}$ & $\begin{array}{l}0.51 \\
0.56\end{array}$ & $\begin{array}{l}0.38 \\
0.38\end{array}$ & $\begin{array}{l}13.87 \\
20.16\end{array}$ & $\begin{array}{l}3.62 \\
3.80\end{array}$ \\
\hline ACEX & ietween PETM and ETM & 1260000 & 195000 & 176000 & 27600 & 1180000 & 50000 & 0.44 & 0.07 & 0.06 & 0.01 & 0.41 & 0.02 & 0.57 & 0.38 & 19.48 & 3.99 \\
\hline ACEX & ietween PETM and ETM & 1250000 & 224000 & 248000 & 31400 & 1370000 & 75300 & 0.39 & 0.07 & 0.08 & 0.01 & 0.43 & & 0.61 & 0.49 & 23.24 & 4.19 \\
\hline ACEX & ietween PETM and ETM & 6990000 & 1030000 & 1020000 & 105000 & 6930000 & 262000 & 0.43 & 0.06 & 0.06 & 0.01 & 42 & 2 & 0.57 & 0.43 & 21.19 & 4.00 \\
\hline ACEX & ietween PETM and ETM & 531000 & 77700 & 61800 & $\begin{array}{l}10900 \\
-2710\end{array}$ & 476000 & 19100 & 0.45 & 0.07 & 0.05 & 0.01 & 0.40 & 0.02 & 0.54 & 0.25 & 17.26 & 3.77 \\
\hline $\begin{array}{l}\text { ACEX } \\
\text { ACEX }\end{array}$ & $\begin{array}{l}\text { etween PETM and ETM } \\
\text { setween PTM and ETM }\end{array}$ & $\begin{array}{l}14500000 \\
576000\end{array}$ & $\begin{array}{l}186000 \\
86400\end{array}$ & $\begin{array}{l}1520000 \\
73300\end{array}$ & $\begin{array}{l}37100 \\
13000\end{array}$ & $\begin{array}{l}1180000 \\
505000\end{array}$ & $\begin{array}{l}43600 \\
23400\end{array}$ & $\begin{array}{l}0.48 \\
0.45\end{array}$ & $\begin{array}{l}0.06 \\
0.07\end{array}$ & $\begin{array}{l}0.05 \\
0.06\end{array}$ & $\begin{array}{l}0.01 \\
0.01\end{array}$ & 0.39 & 003 & 66 & 0.74 & 10.69 & 4.72 \\
\hline $\begin{array}{l}\text { ACEX } \\
\text { ACEX }\end{array}$ & $\begin{array}{l}\text { etween PETM and ETM } \\
\text { etween PETM and ETM }\end{array}$ & $\begin{array}{l}576000 \\
1450000\end{array}$ & $\begin{array}{l}86600 \\
231000\end{array}$ & $\begin{array}{l}73300 \\
225000\end{array}$ & $\begin{array}{l}13900 \\
32500\end{array}$ & $\begin{array}{l}505000 \\
1350000\end{array}$ & $\begin{array}{l}23300 \\
58800\end{array}$ & $\begin{array}{l}0.45 \\
0.43\end{array}$ & $\begin{array}{l}0.07 \\
0.07\end{array}$ & $\begin{array}{l}0.06 \\
0.07\end{array}$ & $\begin{array}{l}0.01 \\
0.01\end{array}$ & $\begin{array}{l}0.40 \\
0.40\end{array}$ & $\begin{array}{l}0.02 \\
0.02\end{array}$ & $\begin{array}{l}0.56 \\
0.58\end{array}$ & $\begin{array}{l}0.51 \\
0.52\end{array}$ & $\begin{array}{l}16.43 \\
20.92\end{array}$ & 4.27 \\
\hline $\begin{array}{l}\text { ACEX } \\
\text { ACEX }\end{array}$ & $\begin{array}{l}\text { etween PETM and ETM } \\
\text { etween PETM and ETM }\end{array}$ & $\begin{array}{l}\begin{array}{l}1450000 \\
2320000\end{array} \\
2\end{array}$ & $\begin{array}{l}231000 \\
363000\end{array}$ & $\begin{array}{l}2250000 \\
374000\end{array}$ & $\begin{array}{l}32500 \\
49200\end{array}$ & $\begin{array}{l}\begin{array}{l}13550000 \\
2460000\end{array} \\
\end{array}$ & $\begin{array}{l}58800 \\
97700\end{array}$ & $\begin{array}{l}0.43 \\
0.41\end{array}$ & $\begin{array}{l}0.07 \\
0.06\end{array}$ & $\begin{array}{l}0.07 \\
0.07\end{array}$ & $\begin{array}{l}0.01 \\
0.01\end{array}$ & $\begin{array}{l}0.40 \\
0.43\end{array}$ & $\begin{array}{l}0.02 \\
0.02\end{array}$ & $\begin{array}{l}0.58 \\
0.59\end{array}$ & $\begin{array}{l}0.52 \\
0.45\end{array}$ & $\begin{array}{l}20.92 \\
21.42\end{array}$ & $\begin{array}{l}4.24 \\
4.04\end{array}$ \\
\hline ACEX & ietween PETM and ETM & 803000 & 118000 & 111000 & 16300 & 800000 & 36100 & 0.43 & 0.06 & 0.06 & 0.01 & 0.42 & 0.02 & 0.58 & 0.42 & 20.12 & $\begin{array}{l}3.04 \\
3.89\end{array}$ \\
\hline ACEX & ietween PETM and ETM & 731000 & 102000 & 83 & 12700 & 672000 & 25400 & 0.45 & 0.06 & 0.05 & 0.01 & 0.41 & 0.02 & 0.54 & 0.36 & 18.37 & 3.69 \\
\hline ACEX & ietween PETM and ETM & 1360000 & 175000 & 122000 & 26400 & 1170000 & 35 & 0.47 & 0. & 0.04 & 0.01 & & & & 37 & 13.99 & 3.71 \\
\hline ACEX & ietween PETM and ETM & 178000 & 25800 & 22900 & $\begin{array}{r}9030 \\
17000\end{array}$ & 175 & 527 & 0.43 & 0.06 & 0.06 & 0.02 & 0.42 & 1 & 0.59 & 0.29 & 17.85 & 4.75 \\
\hline $\begin{array}{l}\text { ACEX } \\
\text { ACEX }\end{array}$ & etween PETM and ETM & $\begin{array}{l}282000 \\
16000\end{array}$ & $\begin{array}{l}38000 \\
21500\end{array}$ & 29200 & 17000 & 236 & $\begin{array}{l}5570 \\
3370\end{array}$ & 0.46 & 0.06 & 0.05 & 0.03 & 0.39 & 0.01 & .58 & 1.33 & 17.39 & 8.28 \\
\hline $\begin{array}{l}\text { ACEX } \\
\text { ACEX }\end{array}$ & $\begin{array}{l}\text { ietween PETM and ETM } \\
\text { etween PETM and ETM }\end{array}$ & $\begin{array}{l}136000 \\
667000\end{array}$ & $\begin{array}{l}21500 \\
79800\end{array}$ & $\begin{array}{l}18800 \\
68800\end{array}$ & $\begin{array}{r}8810 \\
20300\end{array}$ & $\begin{array}{l}1720000 \\
636000\end{array}$ & $\begin{array}{l}3370 \\
16300\end{array}$ & $\begin{array}{l}0.43 \\
0.45\end{array}$ & $\begin{array}{l}0.07 \\
0.05\end{array}$ & $\begin{array}{l}0.06 \\
0.05\end{array}$ & $\begin{array}{l}0.03 \\
0.01\end{array}$ & $\begin{array}{l}0.40 \\
0.43\end{array}$ & $\begin{array}{l}0.01 \\
0.01\end{array}$ & $\begin{array}{l}0.59 \\
0.57\end{array}$ & $\begin{array}{l}1.29 \\
0.71\end{array}$ & $\begin{array}{l}18.00 \\
10.71\end{array}$ & $\begin{array}{l}8.18 \\
4.65\end{array}$ \\
\hline $\begin{array}{l}\text { ACEX } \\
\text { ACEX }\end{array}$ & $\begin{array}{l}\text { ietween PETM and ETM } \\
\text { etween PETM and ETM }\end{array}$ & $\begin{array}{l}666000 \\
471000\end{array}$ & 73400 & $\begin{array}{l}68800 \\
57200\end{array}$ & $\begin{array}{l}20300 \\
15400\end{array}$ & $\begin{array}{l}6360000 \\
491000\end{array}$ & $\begin{array}{l}16300 \\
15000\end{array}$ & $\begin{array}{l}0.45 \\
0.42\end{array}$ & $\begin{array}{l}0.05 \\
0.07\end{array}$ & $\begin{array}{l}0.05 \\
0.05\end{array}$ & $\begin{array}{l}0.01 \\
0.01\end{array}$ & $\begin{array}{l}0.43 \\
0.44\end{array}$ & $\begin{array}{l}0.01 \\
0.01\end{array}$ & 0.54 & $\begin{array}{l}0.71 \\
0.51\end{array}$ & $\begin{array}{l}10.11 \\
15.03\end{array}$ & $\begin{array}{l}4.65 \\
4.26\end{array}$ \\
\hline ACEX & $\begin{array}{l}\text { PETM } \\
\end{array}$ & 270000 & 27500 & 20400 & 9660 & 224000 & 5280 & 0.48 & 0.05 & 0.04 & 0.02 & $\begin{array}{l}0.44 \\
0.40\end{array}$ & 0.01 & 0.56 & 0.39 & $\begin{array}{l}3.035 \\
3.78\end{array}$ & $\begin{array}{l}4.26 \\
4.34\end{array}$ \\
\hline ACEX & PE & 223000 & 21800 & 13 & 71 & 182 & 38 & 0.49 & $0 . c$ & 0 & 0.02 & 0.40 & 0 & 0.53 & 0.44 & 1.92 & 4.30 \\
\hline ACEX & PETM & 404000 & 52400 & 38800 & 16100 & 366 & 85 & 0.46 & 0. & 0.04 & 0.02 & 0.41 & 0.01 & 0.55 & 0.68 & 9.59 & 4.88 \\
\hline ACEX & PETM & 308000 & 29600 & $\begin{array}{l}19800 \\
52000\end{array}$ & 11100 & $\begin{array}{l}232000 \\
555000\end{array}$ & 4870 & 0.51 & 0.05 & 0.03 & 0.02 & 0.38 & 0.01 & 0.55 & 0.23 & 2.62 & 4.45 \\
\hline $\begin{array}{l}\text { ACEX } \\
\end{array}$ & PETM & $\begin{array}{l}672000 \\
2000\end{array}$ & $\begin{array}{l}70200 \\
43500\end{array}$ & $\begin{array}{l}52200 \\
31100\end{array}$ & $\begin{array}{l}21800 \\
1800\end{array}$ & 558000 & 12800 & 0.48 & 0.05 & 0.04 & 0.02 & $\begin{array}{l}0.40 \\
\end{array}$ & 0.01 & 0.55 & 0.52 & $\begin{array}{r}3.60 \\
1.325\end{array}$ & $\begin{array}{l}4.53 \\
5.53\end{array}$ \\
\hline $\begin{array}{l}\text { ACEX } \\
\text { ACEX }\end{array}$ & $\begin{array}{l}\text { PETM } \\
\text { PETM }\end{array}$ & $\begin{array}{l}278000 \\
437000\end{array}$ & $\begin{array}{l}43500 \\
69100\end{array}$ & $\begin{array}{l}311200 \\
54800\end{array}$ & $\begin{array}{l}11600 \\
14900\end{array}$ & $\begin{array}{l}233000 \\
473000\end{array}$ & $\begin{array}{c}6480 \\
12900\end{array}$ & $\begin{array}{l}0.46 \\
0.41\end{array}$ & $\begin{array}{l}0.07 \\
0.07\end{array}$ & $\begin{array}{l}0.05 \\
0.05\end{array}$ & $\begin{array}{l}0.02 \\
0.01\end{array}$ & $\begin{array}{l}0.39 \\
0.45\end{array}$ & $\begin{array}{l}0.01 \\
0.01\end{array}$ & $\begin{array}{l}0.53 \\
0.54\end{array}$ & $\begin{array}{l}0.73 \\
0.42\end{array}$ & $\begin{array}{l}13.35 \\
16.08\end{array}$ & $\begin{array}{l}5.51 \\
4.22\end{array}$ \\
\hline $\begin{array}{l}\text { ACEX } \\
\text { ACEX }\end{array}$ & $\begin{array}{l}\text { PETM } \\
\text { PETM }\end{array}$ & 321000 & $\begin{array}{l}69100 \\
53600\end{array}$ & $\begin{array}{l}58400 \\
38900\end{array}$ & $\begin{array}{l}14400 \\
14500\end{array}$ & $\begin{array}{l}473 \\
309\end{array}$ & 9090 & $\begin{array}{l}0.41 \\
0.43\end{array}$ & 0.07 & $\begin{array}{l}0.05 \\
0.05\end{array}$ & 0.02 & 0.41 & 0.01 & $\begin{array}{l}0.54 \\
0.54\end{array}$ & $\begin{array}{l}0.42 \\
0.68\end{array}$ & $\begin{array}{l}16.08 \\
16.61\end{array}$ & $\begin{array}{l}4.22 \\
5.29\end{array}$ \\
\hline ACEX & PETM & 354000 & 52300 & 38300 & 13500 & 345000 & 8790 & 0.44 & 0.06 & 0.05 & 0.02 & 0.42 & 0.01 & 0.54 & 0.68 & 12.28 & 4.94 \\
\hline ACEX & РЕTM & 197000 & 38700 & 36600 & 10200 & 241000 & 7470 & 0.37 & 0.07 & 0.07 & 0.02 & 0.45 & 0.01 & 0.58 & 0.96 & 21.75 & 5.27 \\
\hline
\end{tabular}


Eocene dataset for OPTiMAL

\begin{tabular}{|c|c|c|c|c|c|c|c|c|c|c|c|c|c|c|c|c|c|}
\hline SITE & AGE & $\begin{array}{c}1302 \\
\text { GDGTO }\end{array}$ & $\begin{array}{c}1300 \\
\text { GDGT1 }\end{array}$ & $\begin{array}{c}1298 \\
\text { GDGT2 }\end{array}$ & $\begin{array}{c}1296 \\
\text { GDGT3 }\end{array}$ & $\begin{array}{l}1292 \\
\text { CREN }\end{array}$ & $\begin{array}{l}1292^{\prime} \\
\text { CREN }\end{array}$ & Fr. 1302 & Fr. 1300 & Fr.1298 & Fr.1296 & Fr.1292 & Fr.1292' & $\begin{array}{c}\text { TEX } \\
\text { (published) }\end{array}$ & D_nearest & $\begin{array}{c}\text { OPTTMAL } \\
\text { SST }\end{array}$ & $\begin{array}{c}\text { OPTIMAL } \\
\text { StDev } \\
\end{array}$ \\
\hline ACEX & PETM & 304000 & 54000 & 58100 & 17000 & 369000 & 13000 & 0.37 & 0.07 & 0.07 & 0.02 & 0.45 & 0.02 & 0.62 & 0.85 & 21.28 & 5.55 \\
\hline ACEX & РЕТМ & 400000 & 72700 & 64900 & 21700 & 506000 & 14800 & 0.37 & 0.07 & 0.06 & 0.02 & 0.47 & 0.01 & 0.58 & 0.93 & 20.49 & 5.25 \\
\hline ACEX & PETM & 441000 & 86700 & 85800 & 28200 & 709000 & 21600 & 0.32 & 0.06 & 0.06 & 0.02 & 0.52 & 0.02 & 0.61 & 0.71 & 20.39 & 4.96 \\
\hline ACEX & PETM & 306000 & 45000 & 42500 & 17600 & 457000 & 11700 & 0.35 & 0.05 & 0.05 & 0.02 & 0.52 & 0.01 & 0.61 & 0.96 & 18.03 & 5.80 \\
\hline ACEX & PETM & 241000 & 42600 & 35500 & 12800 & 410000 & 10600 & 0.32 & 0.06 & 0.05 & 0.02 & 0.54 & 0.01 & 0.58 & 0.25 & 19.33 & 4.00 \\
\hline ACEX & PETM & 249000 & 46300 & 45900 & 17300 & 503000 & 16000 & 0.28 & 0.05 & 0.05 & 0.02 & 0.57 & 0.02 & 0.63 & 0.59 & 19.67 & 4.76 \\
\hline $\begin{array}{l}\text { ACEX } \\
\text { ACEX }\end{array}$ & $\begin{array}{l}\text { PETM } \\
\text { PETM }\end{array}$ & 646000 & $\begin{array}{l}131000 \\
37300\end{array}$ & 126000 & 49200 & 1340000 & 41500 & 0.28 & 0.06 & 0.05 & 0.02 & 0.57 & 0.02 & 0.62 & 0.52 & 19.75 & 4.81 \\
\hline ACEX & PETM & 214000 & 37300 & 37000 & 13200 & 400000 & 11900 & 0.30 & 0.05 & 0.05 & 0.02 & 0.56 & 0.02 & 0.62 & 0.49 & 19.50 & 4.56 \\
\hline ACEX & PETM & 348000 & 62200 & 64200 & 23500 & 685000 & 23600 & 0.29 & 0.05 & 0.05 & 0.02 & 0.57 & 0.02 & 0.64 & 0.61 & 19.80 & 4.88 \\
\hline ACEX & PETM & 151000 & 30100 & 27600 & 11200 & 290000 & 8330 & 0.29 & 0.06 & 0.05 & 0.02 & 0.56 & 0.02 & 0.61 & 0.43 & 18.99 & 4.89 \\
\hline ACEX & PETM & 204000 & 36000 & 35400 & 13900 & 395000 & 10900 & 0.29 & 0.05 & 0.05 & 0.02 & 0.57 & 0.02 & 0.63 & 0.67 & 18.81 & 5.01 \\
\hline ACEX & PETM & 266000 & 41400 & 41500 & 16200 & 463000 & 14200 & 0.32 & 0.05 & 0.05 & 0.02 & 0.55 & 0.02 & 0.63 & 0.73 & 18.64 & 5.33 \\
\hline ACEX & PETM & 447000 & 80000 & 78700 & 28800 & 845000 & 25000 & 0.30 & 0.05 & 0.05 & 0.02 & 0.56 & 0.02 & 0.62 & 0.56 & 19.41 & 4.66 \\
\hline 913 & 35.86 & 44576470 & 8328164 & 9417640 & 907768 & 49985696 & 4211650 & 0.38 & 0.07 & 0.08 & 0.01 & 0.43 & 0.04 & 0.64 & 0.52 & 24.73 & 4.14 \\
\hline 913 & 36.27 & $\begin{array}{l}6890376 \\
53201670\end{array}$ & 1144017 & 1104032 & $\begin{array}{l}1141222 \\
0508123\end{array}$ & $\begin{array}{l}6922606 \\
57170120\end{array}$ & 443907 & 0.41 & 0.07 & 0.07 & 0.01 & 0.42 & 0.03 & 0.59 & 0.50 & 22.18 & 3.90 \\
\hline $\begin{array}{l}913 \\
913\end{array}$ & 36.53 & $\begin{array}{r}53091672 \\
\end{array}$ & $\begin{array}{c}9368211 \\
17147320\end{array}$ & 9241054 & $\begin{array}{r}958133 \\
\end{array}$ & $\begin{array}{l}54796136 \\
11102077\end{array}$ & 4081748 & 0.40 & 0.07 & 0.07 & 0.01 & 0.42 & 0.03 & 0.60 & 0.49 & 23.17 & 3.92 \\
\hline $\begin{array}{l}913 \\
913\end{array}$ & $\begin{array}{l}36.60 \\
37.21\end{array}$ & $\begin{array}{c}1000744668 \\
56034512\end{array}$ & $\begin{array}{c}1717477320 \\
8776230\end{array}$ & $\begin{array}{c}18184278 \\
8507421\end{array}$ & $\begin{array}{c}1731557 \\
831286\end{array}$ & $\begin{array}{l}11110209776 \\
46583224\end{array}$ & $\begin{array}{l}6905992 \\
3499627\end{array}$ & $\begin{array}{l}0.39 \\
0.45\end{array}$ & $\begin{array}{l}0.07 \\
0.07\end{array}$ & $\begin{array}{l}0.07 \\
0.07\end{array}$ & $\begin{array}{l}0.01 \\
0.01\end{array}$ & $\begin{array}{l}0.43 \\
0.37\end{array}$ & $\begin{array}{l}0.03 \\
0.03\end{array}$ & $\begin{array}{l}0.61 \\
0.59\end{array}$ & 0.48 & $\begin{array}{l}22.98 \\
22.98\end{array}$ & 3.98 \\
\hline $\begin{array}{l}913 \\
913\end{array}$ & $\begin{array}{l}37.21 \\
37.47\end{array}$ & $\begin{array}{c}56034512 \\
168004\end{array}$ & $\begin{array}{c}87762300 \\
29740\end{array}$ & $\begin{array}{c}85072421 \\
25849\end{array}$ & $\begin{array}{c}831286 \\
2415\end{array}$ & $\begin{array}{c}465683224 \\
193216\end{array}$ & $\begin{array}{c}3493627 \\
13431\end{array}$ & $\begin{array}{l}0.45 \\
0.39\end{array}$ & $\begin{array}{l}0.07 \\
0.07\end{array}$ & $\begin{array}{l}0.07 \\
0.06\end{array}$ & $\begin{array}{l}0.01 \\
0.01\end{array}$ & $\begin{array}{l}0.37 \\
0.45\end{array}$ & $\begin{array}{l}0.03 \\
0.03\end{array}$ & $\begin{array}{l}0.59 \\
0.58\end{array}$ & $\begin{array}{l}0.47 \\
0.35\end{array}$ & $\begin{array}{l}22.88 \\
20.73\end{array}$ & $\begin{array}{l}4.21 \\
3.58\end{array}$ \\
\hline 913 & 37.88 & 45748195 & 7174086 & 6217027 & 844961 & 50059292 & 2998621 & 0.40 & 0.06 & 0.05 & 0.01 & 0.44 & 0.03 & 0.58 & $\begin{array}{l}0.37 \\
0.37\end{array}$ & 20.06 & $\begin{array}{l}3.58 \\
3.61\end{array}$ \\
\hline 913 & 37.99 & 22726800 & 3902315 & 3296297 & 335639 & 16642844 & 1200340 & 0.47 & 0.08 & 0.07 & 0.01 & 0.35 & 0.02 & 0.55 & 0.38 & 22.78 & $\begin{array}{l}3.61 \\
4.07\end{array}$ \\
\hline 913 & 38.16 & 32221629 & 5530647 & 4261322 & 440398 & 34149808 & 2183935 & 0.41 & 0.07 & 0.05 & 0.01 & 0.43 & 0.03 & 0.55 & 0.32 & 18.29 & 3.54 \\
\hline 913 & 38.28 & 9584836 & 1526568 & 1109356 & 91793 & 5410909 & 380441 & 0.53 & 0.08 & 0.06 & 0.01 & 0.30 & 0.02 & 0.51 & 0.68 & 15.87 & 4.49 \\
\hline 913 & 38.60 & 22452614 & 4463983 & 3748336 & 367957 & 19762438 & 1745071 & 0.43 & 0.08 & 0.07 & 0.01 & 0.38 & 0.03 & 0.57 & 0.39 & 22.43 & 4.01 \\
\hline 913 & 39.63 & 387530 & 46022 & 31462 & 3432 & 163385 & 10952 & 0.60 & 0.07 & 0.05 & 0.01 & 0.25 & 0.02 & 0.50 & 0.56 & 11.75 & 4.39 \\
\hline 913 & 39.69 & 2921030 & 227384 & 224845 & 18594 & 475567 & 43394 & 0.75 & 0.06 & 0.06 & 0.00 & 0.12 & 0.01 & 0.56 & 1.17 & 11.38 & 7.21 \\
\hline 913 & 40.01 & 21787654 & 2966410 & 2426934 & 309836 & 17309324 & 1292353 & 0.47 & 0.06 & 0.05 & 0.01 & 0.38 & 0.03 & 0.58 & 0.33 & 18.55 & 3.85 \\
\hline 913 & 40.13 & 84060898 & 14490079 & 13780120 & 1359260 & 82842848 & 6537485 & 0.41 & 0.07 & 0.07 & 0.01 & 0.41 & 0.03 & 0.60 & 0.52 & 22.66 & 3.95 \\
\hline 913 & 40.30 & 170353 & 54768 & 17578 & 1407 & 58378 & 4546 & 0.55 & 0.18 & 0.06 & 0.00 & 0.19 & 0.01 & 0.30 & 2.79 & 15.47 & 8.90 \\
\hline 913 & 41.40 & 75817696 & 15240714 & 17597154 & 1850374 & 111595012 & 10218329 & 0.33 & 0.07 & 0.08 & 0.01 & 0.48 & 0.04 & 0.66 & 0.31 & 22.28 & 3.65 \\
\hline 913 & 41.79 & 49396877 & 9953333 & $\begin{array}{l}11984820 \\
5230602\end{array}$ & 1435266 & 75044140 & 5982444 & 0.32 & 0.06 & 0.08 & 0.01 & 0.49 & 0.04 & 0.66 & 0.33 & 22.04 & 3.60 \\
\hline $\begin{array}{l}913 \\
913\end{array}$ & $\begin{array}{l}42.31 \\
43.55\end{array}$ & 35586708 & 5783732 & 5336602 & $\begin{array}{l}661690 \\
\end{array}$ & 44389373 & 3353989 & 0.37 & 0.06 & 0.06 & 0.01 & 0.47 & 0.04 & 0.62 & 0.25 & 20.81 & 3.61 \\
\hline $\begin{array}{l}913 \\
913\end{array}$ & $\begin{array}{l}43.55 \\
43.98\end{array}$ & $\begin{array}{l}41558577 \\
16903132\end{array}$ & $\begin{array}{l}10489195 \\
3050618\end{array}$ & $\begin{array}{l}14217098 \\
2814344\end{array}$ & $\begin{array}{l}1625232 \\
471878\end{array}$ & $\begin{array}{l}84779494 \\
25449972\end{array}$ & $\begin{array}{r}7534865 \\
1766424\end{array}$ & $\begin{array}{l}0.26 \\
0.34\end{array}$ & $\begin{array}{l}0.07 \\
0.06\end{array}$ & $\begin{array}{l}0.09 \\
0.06\end{array}$ & 0.01 & $\begin{array}{l}0.53 \\
0.50\end{array}$ & 0.05 & 0.69 & 0.29 & $\begin{array}{l}25.24 \\
\end{array}$ & 3.50 \\
\hline $\begin{array}{l}\begin{array}{l}913 \\
913\end{array}\end{array}$ & $\begin{array}{l}43.98 \\
44.66\end{array}$ & $\begin{array}{c}119603132 \\
574026\end{array}$ & $\begin{array}{l}30056618 \\
125370\end{array}$ & $\begin{array}{c}28143444 \\
136948\end{array}$ & $\begin{array}{l}417878 \\
15873\end{array}$ & $\begin{array}{c}255449872 \\
830854\end{array}$ & $\begin{array}{c}1766424 \\
74652\end{array}$ & $\begin{array}{l}0.34 \\
0.33\end{array}$ & $\begin{array}{l}0.06 \\
0.07\end{array}$ & $\begin{array}{l}0.06 \\
0.08\end{array}$ & $\begin{array}{l}0.01 \\
0.01\end{array}$ & $\begin{array}{l}0.50 \\
0.47\end{array}$ & $\begin{array}{l}0.04 \\
0.04\end{array}$ & $\begin{array}{l}0.62 \\
0.64\end{array}$ & $\begin{array}{l}0.19 \\
0.29\end{array}$ & $\begin{array}{l}19.87 \\
23.40\end{array}$ & $\begin{array}{l}3.46 \\
3.53\end{array}$ \\
\hline South Dover Bridge & 41.45 & 631090347 & 250934590 & 259894750 & 143331291 & 1830058006 & 133757639 & 0.19 & 0.08 & 0.08 & 0.04 & $\begin{array}{l}0.41 \\
0.56\end{array}$ & $\begin{array}{l}0.04 \\
0.04\end{array}$ & $\begin{array}{l}0.64 \\
0.68\end{array}$ & $\begin{array}{l}0.19 \\
1.10\end{array}$ & $\begin{array}{l}23.40 \\
23.35\end{array}$ & $\begin{array}{l}3.53 \\
7.49\end{array}$ \\
\hline South Dover Bridge & 41.77 & 93582437 & 32063068 & 32378905 & 15980422 & 255918611 & 19358572 & 0.21 & 0.07 & 0.07 & 0.04 & 0.57 & 0.04 & 0.68 & 0.56 & 23.14 & $\begin{array}{l}. .49 \\
5.97\end{array}$ \\
\hline South Dover Bridge & 41.99 & 812722801 & 318757578 & 323986559 & 189013738 & 2344544767 & 189499893 & 0.19 & 0.08 & 0.08 & 0.05 & 0.56 & 0.05 & 0.69 & 1.11 & 23.12 & 7.45 \\
\hline South Dover Bridge & 42.20 & 1104411664 & 343861929 & 397893290 & 216979426 & 2375373968 & 230584690 & 0.24 & 0.07 & 0.09 & 0.05 & 0.51 & 0.05 & 0.71 & 1.41 & 20.72 & 8.20 \\
\hline South Dover Bridge & 42.44 & 688031310 & 249975718 & 271870744 & 156165695 & 1741421491 & 161455372 & 0.21 & 0.08 & 0.08 & 0.05 & 0.53 & 0.05 & 0.70 & 1.12 & 21.51 & 7.72 \\
\hline South Dover Bridge & 42.71 & 447175311 & 203630240 & 264697153 & 140570131 & 1714299972 & 192868995 & 0.15 & 0.07 & 0.09 & 0.0 & & 0.07 & 0.7 & 0.78 & 24.05 & 6.53 \\
\hline South Dover Bridge & 43.61 & 368063515 & 158615858 & 198376222 & 112527292 & 1393543898 & 144347473 & 0.15 & 0.07 & 0.08 & 0.05 & 0.59 & 0.06 & 0.74 & 0.75 & 24.70 & 6.28 \\
\hline South Dover Bridge & 44.62 & 618578980 & 268354811 & 325996667 & 176951487 & 2162293435 & 233883560 & 0.16 & 0.07 & 0.09 & 0.05 & 0.57 & 0.06 & 0.73 & 0.90 & 23.86 & 6.76 \\
\hline South Dover Bridge & 45.83 & 297286057 & 126033144 & 150200353 & 75941378 & 1134979963 & 82103959 & 0.16 & 0.07 & 0.08 & 0.04 & 0.61 & 0.04 & 0.71 & 0.74 & 25.74 & 5.92 \\
\hline South Dover Bridge & 46.99 & 327765781 & 120997531 & 153932798 & 91808850 & 1205093151 & 127282447 & 0.16 & 0.06 & 0.08 & 0.05 & 0.59 & 0.06 & 0.76 & 0.70 & 25.11 & 6.27 \\
\hline South Dover Bridge & 49.07 & 332019599 & 166669336 & $\begin{array}{l}256268595 \\
76677727\end{array}$ & 139380682 & 1631876927 & 192173277 & 0.12 & 0.06 & 0.09 & 0.05 & 0.60 & 0 & 0.78 & 0.82 & 24.18 & 6.74 \\
\hline South Dover Bridge & 50.31 & 99869098 & 50827785 & 76777727 & 39564776 & 510268312 & 67989856 & 0.12 & & 0.09 & 0.05 & 0.60 & 08 & 0.78 & 1.02 & 23.25 & 7.29 \\
\hline $\begin{array}{l}\text { South Dover Bridge } \\
\text { South Dover Bridge }\end{array}$ & 50.74 & $\begin{array}{l}1476637 \\
55952109\end{array}$ & 651633 & $\begin{array}{l}852462 \\
\end{array}$ & $\begin{array}{l}553640 \\
\end{array}$ & $\begin{array}{l}9687308 \\
06701027\end{array}$ & 1206009 & 0.10 & 0.05 & 0.06 & 0.04 & 0.67 & 0.08 & 0.80 & 1.49 & 20.12 & 8.40 \\
\hline $\begin{array}{l}\text { South Dover Bridge } \\
\text { South Dover Bridge }\end{array}$ & $\begin{array}{l}51.47 \\
52.26\end{array}$ & $\begin{array}{l}5585524193 \\
397170268\end{array}$ & $\begin{array}{l}\begin{array}{l}2810124247 \\
211527521\end{array} \\
2152\end{array}$ & $\begin{array}{l}\begin{array}{l}4811170584 \\
355766291\end{array} \\
\text { ? }\end{array}$ & $\begin{array}{l}2499955333 \\
175441158\end{array}$ & 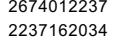 & $\begin{array}{l}385701576 \\
310332203\end{array}$ & $\begin{array}{l}0.12 \\
0.11\end{array}$ & 0.06 & $\begin{array}{l}0.10 \\
0.10\end{array}$ & $\begin{array}{l}0.05 \\
0.05\end{array}$ & $\begin{array}{l}0.58 \\
0.61\end{array}$ & 0.08 & 0.80 & $\begin{array}{l}1.20 \\
1.05\end{array}$ & $\begin{array}{l}21.30 \\
22.62\end{array}$ & 7.96 \\
\hline $\begin{array}{l}\text { South Dover Bridge } \\
\text { South Dover Bridge }\end{array}$ & $\begin{array}{l}52.26 \\
54.03\end{array}$ & $\begin{array}{l}397170268 \\
443190011\end{array}$ & $\begin{array}{l}2111527521 \\
174272223\end{array}$ & $\begin{array}{l}3557662911 \\
248151800\end{array}$ & $\begin{array}{l}\begin{array}{l}175541158 \\
120419431\end{array} \\
\end{array}$ & $\begin{array}{l}223311620034 \\
1541093835\end{array}$ & $\begin{array}{l}\begin{array}{l}3103322003 \\
202337195\end{array} \\
205\end{array}$ & $\begin{array}{l}0.11 \\
0.16\end{array}$ & $\begin{array}{l}0.06 \\
0.06\end{array}$ & $\begin{array}{l}0.10 \\
0.09\end{array}$ & $\begin{array}{l}0.05 \\
0.04\end{array}$ & $\begin{array}{l}0.61 \\
0.56\end{array}$ & $\begin{array}{l}0.08 \\
0.07\end{array}$ & $\begin{array}{l}0.80 \\
0.77\end{array}$ & $\begin{array}{l}1.05 \\
0.96\end{array}$ & $\begin{array}{l}22.62 \\
23.59\end{array}$ & 7.58 \\
\hline South Dover Bridge & 54.65 & 244464780 & $\begin{array}{l}11421992109 \\
11192\end{array}$ & $\begin{array}{l}248518000 \\
178504295\end{array}$ & 85183888 & $\begin{array}{l}154010398353 \\
1161533034\end{array}$ & 152645880 & $\begin{array}{l}0.16 \\
0.13\end{array}$ & $\begin{array}{l}0.06 \\
0.06\end{array}$ & $\begin{array}{l}0.09 \\
0.09\end{array}$ & $\begin{array}{l}0.04 \\
0.04\end{array}$ & $\begin{array}{l}0.56 \\
0.60\end{array}$ & 0.08 & 0.79 & $\begin{array}{l}0.96 \\
0.99\end{array}$ & $\begin{array}{l}23.59 \\
23.07\end{array}$ & $\begin{array}{l}7.28 \\
7.43\end{array}$ \\
\hline Seymour Island & 37.40 & 7314084 & 496162 & 241649 & 93928 & 7901663 & 146629 & 0.45 & 0.03 & 0.01 & 0.01 & 0.49 & 0.01 & 0.49 & 0.19 & 8.51 & 3.43 \\
\hline Seymour Island & 37.50 & 5530598 & 378895 & 143855 & 71918 & 6245 & 744 & 0.44 & 3 & 0.01 & 0. & 0.50 & 0.01 & 0.43 & 0.20 & 9.03 & 3.42 \\
\hline Seymour Island & 38.40 & 22 & 128925 & 70439 & 28631 & 1904370 & 291 & 0.50 & & 0. & 0. & & & & 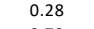 & & 3.43 \\
\hline Seymour Island & $\begin{array}{l}43.50 \\
\text { PFTM }\end{array}$ & 2428920 & 229762 & 175734 & 73174 & 1640330 & 71234 & 0.53 & 0.05 & 0.04 & 0.02 & 6 & 2 & 0.58 & 0.73 & 2.30 & 5.08 \\
\hline $\begin{array}{l}\text { Wilson Lake } \\
\text { Wilison Lake }\end{array}$ & $\begin{array}{l}\text { PETM } \\
\text { PETM }\end{array}$ & $\begin{array}{l}0.18 \\
0.18\end{array}$ & $\begin{array}{l}0.05 \\
0.07\end{array}$ & $\begin{array}{l}0.08 \\
0.09\end{array}$ & $\begin{array}{l}0.04 \\
0.04\end{array}$ & $\begin{array}{l}0.56 \\
0.56\end{array}$ & $\begin{array}{l}0.08 \\
0.07\end{array}$ & $\begin{array}{l}0.18 \\
0.18\end{array}$ & $\begin{array}{l}0.05 \\
0.07\end{array}$ & $\begin{array}{l}0.08 \\
0.09\end{array}$ & $\begin{array}{l}0.04 \\
0.04\end{array}$ & 0.56 & $\begin{array}{l}0.08 \\
0.07\end{array}$ & $\begin{array}{l}0.79 \\
0.74\end{array}$ & $\begin{array}{l}0.95 \\
0.98\end{array}$ & $\begin{array}{l}22.28 \\
22.03\end{array}$ & $\begin{array}{l}7.42 \\
77.18\end{array}$ \\
\hline $\begin{array}{l}\text { Wison Lake } \\
\text { Wilson Lake }\end{array}$ & $\begin{array}{l}\text { PETM } \\
\text { PETM }\end{array}$ & $\begin{array}{l}0.18 \\
0.16\end{array}$ & $\begin{array}{l}0.07 \\
0.07\end{array}$ & $\begin{array}{l}0.09 \\
0.10\end{array}$ & $\begin{array}{l}0.04 \\
0.05\end{array}$ & $\begin{array}{l}0.56 \\
0.57\end{array}$ & $\begin{array}{l}0.07 \\
0.07\end{array}$ & $\begin{array}{l}0.18 \\
0.16\end{array}$ & $\begin{array}{l}0.07 \\
0.07\end{array}$ & $\begin{array}{l}0.09 \\
0.10\end{array}$ & $\begin{array}{l}0.04 \\
0.05\end{array}$ & $\begin{array}{l}0.56 \\
0.57\end{array}$ & $\begin{array}{l}0.07 \\
0.07\end{array}$ & $\begin{array}{l}0.74 \\
0.77\end{array}$ & $\begin{array}{l}0.98 \\
1.02\end{array}$ & $\begin{array}{l}24.03 \\
23.41\end{array}$ & 7.18 \\
\hline $\begin{array}{l}\text { Wisison Lake } \\
\text { Wison Lake }\end{array}$ & $\begin{array}{l}\text { PETM } \\
\text { PETM }\end{array}$ & $\begin{array}{l}0.16 \\
0.26\end{array}$ & $\begin{array}{l}0.07 \\
0.07\end{array}$ & $\begin{array}{l}0.10 \\
0.07\end{array}$ & $\begin{array}{l}0.05 \\
0.03\end{array}$ & $\begin{array}{l}0.57 \\
0.51\end{array}$ & $\begin{array}{l}0.07 \\
0.06\end{array}$ & $\begin{array}{l}0.16 \\
0.26\end{array}$ & $\begin{array}{l}0.07 \\
0.07\end{array}$ & $\begin{array}{l}0.10 \\
0.07\end{array}$ & $\begin{array}{l}0.05 \\
0.03\end{array}$ & $\begin{array}{l}0.57 \\
0.51\end{array}$ & $\begin{array}{l}0.07 \\
0.06\end{array}$ & 0.70 & $\begin{array}{l}1.02 \\
1.50\end{array}$ & $\begin{array}{l}23.41 \\
20.13\end{array}$ & $\begin{array}{l}7.28 \\
8.13\end{array}$ \\
\hline Wilson Lake & $\mathrm{PE}$ & 0.09 & 0.04 & 0.08 & 0.04 & 0.63 & 0.13 & 0.09 & & 0.08 & 0.04 & 0.63 & 0. & 0.87 & 2.14 & 17.80 & $\begin{array}{l}8.13 \\
8.82\end{array}$ \\
\hline Wilson Lake & PE & 0 & 0.04 & 0.0 & 0. & 0. & 0.1 & 0.06 & & 0. & 0. & 0.66 & 0.13 & 0.87 & 1.77 & 18.45 & 8.68 \\
\hline Wilson L & PETM & 0. & 0.0 & 0.0 & 0. & 0. & 0. & 0.05 & & 0. & 0. & 0.6 & & 0.89 & 1.96 & 17.52 & 8.79 \\
\hline Wilson Lake & PETM & 0.05 & 0.03 & 0.08 & 0.04 & 0.66 & 0.1 & 0.05 & & 0.08 & 0.04 & 0.66 & 0.13 & 0.89 & 1.99 & 17.48 & 8.80 \\
\hline Wilson Lake & $\begin{array}{l}\text { PETM } \\
\text { PETM }\end{array}$ & 0.05 & 0.03 & 0.08 & 0.03 & 0.68 & 0.1 & 0.05 & & 0.08 & 0.03 & 8 & 0.13 & 0.89 & 1.20 & 21.16 & 7.88 \\
\hline $\begin{array}{l}\text { Wilison Lake } \\
\text { Wilson Lake }\end{array}$ & $\begin{array}{l}\text { PETM } \\
\text { PETM }\end{array}$ & $\begin{array}{l}0.04 \\
0.06\end{array}$ & $\begin{array}{l}0.02 \\
0.03\end{array}$ & $\begin{array}{l}0.07 \\
0.08\end{array}$ & $\begin{array}{l}0.03 \\
0.03\end{array}$ & $\begin{array}{l}0.68 \\
0.67\end{array}$ & $\begin{array}{l}0.15 \\
0.14\end{array}$ & $\begin{array}{l}0.04 \\
0.06\end{array}$ & $\begin{array}{l}0.02 \\
0.03\end{array}$ & $\begin{array}{l}0.07 \\
0.08\end{array}$ & $\begin{array}{l}0.03 \\
0.03\end{array}$ & $\begin{array}{l}0.68 \\
0.67\end{array}$ & $\begin{array}{l}0.15 \\
0.14\end{array}$ & $\begin{array}{l}0.91 \\
0.89\end{array}$ & $\begin{array}{l}1.21 \\
1.72\end{array}$ & $\begin{array}{l}21.31 \\
18.31\end{array}$ & $\begin{array}{l}7.78 \\
8865\end{array}$ \\
\hline $\begin{array}{l}\text { Wison Le } \\
\text { Wison Lake }\end{array}$ & PETM & $\begin{array}{l}0.06 \\
0.10\end{array}$ & 0.04 & $\begin{array}{l}0.08 \\
0.07\end{array}$ & $\begin{array}{l}0.03 \\
0.03\end{array}$ & $\begin{array}{l}0.67 \\
0.64\end{array}$ & $\begin{array}{l}0.14 \\
0.12\end{array}$ & $\begin{array}{l}0.10 \\
0.10\end{array}$ & 0.0 & $\begin{array}{l}0.08 \\
0.07\end{array}$ & $\begin{array}{l}0.03 \\
0.03\end{array}$ & 0.64 & 0.12 & $\begin{array}{l}0.89 \\
0.86\end{array}$ & $\begin{array}{l}1.12 \\
0.67\end{array}$ & $\begin{array}{l}28.51 \\
25.56\end{array}$ & $\begin{array}{l}8.65 \\
5.98\end{array}$ \\
\hline Wilson Lake & PETM & 0.10 & 0.03 & 0.07 & 0.03 & 0.64 & 0.13 & 0.10 & 0.03 & 0.07 & 0.03 & 0.64 & 0.13 & 0.88 & 1.07 & 22.61 & 7.52 \\
\hline Wilson Lake & РЕТМ & 0.04 & 0.02 & 0.07 & 0.03 & 0.65 & 0.19 & 0.04 & 0.02 & 0.07 & 0.03 & 0.65 & 0.19 & 0.93 & 1.79 & 17.90 & 8.70 \\
\hline
\end{tabular}


Eocene dataset for OPTiMAL

\begin{tabular}{|c|c|c|c|c|c|c|c|c|c|c|c|c|c|c|c|c|c|}
\hline SITE & AGE & $\begin{array}{c}1302 \\
\text { GDGTO } \\
\end{array}$ & $\begin{array}{c}1300 \\
\text { GDGT1 }\end{array}$ & $\begin{array}{c}1298 \\
\text { GDGT2 } \\
\end{array}$ & $\begin{array}{c}1296 \\
\text { GDGT3 } \\
\end{array}$ & $\begin{array}{l}1292 \\
\text { CREN } \\
\end{array}$ & $\begin{array}{l}1292^{\prime} \\
\text { CREN' }^{\prime} \\
\end{array}$ & Fr.1302 & Fr.1300 & Fr.1298 & Fr.1296 & Fr.1292 & Fr.1292' & $\begin{array}{c}\text { TEX } \\
\text { (published) }\end{array}$ & D_nearest & $\begin{array}{c}\text { OPTiMAL } \\
\text { SST }\end{array}$ & $\begin{array}{c}\text { OPTiMAL } \\
\text { StDev }\end{array}$ \\
\hline $\begin{array}{l}\text { Wison Lake } \\
\text { Wol }\end{array}$ & $\begin{array}{l}\text { PETM } \\
\end{array}$ & 0.19 & $\begin{array}{l}0.06 \\
\end{array}$ & 0.09 & 0.03 & 0.55 & 0.08 & 0.19 & 0.06 & 0.09 & 0.03 & 0.55 & 0.08 & 0.78 & 1.40 & 22.60 & 7.82 \\
\hline Wilson Lake & PETM & 0.20 & 0.08 & 0.08 & 0.04 & 0.54 & 0.06 & 0.20 & 0.08 & 0.08 & 0.04 & 0.54 & 0.06 & 0.70 & 0.67 & 23.67 & 6.44 \\
\hline Wilson Lake & PETM & 0.28 & 0.08 & 0.07 & 0.03 & 0.49 & 0.04 & 0.28 & 0.08 & 0.07 & 0.03 & 0.49 & 0.04 & 0.66 & 1.71 & 18.29 & 8.65 \\
\hline Wilson Lake & PETM & 0.18 & 0.08 & 0.09 & 0.05 & 0.55 & 0.07 & 0.18 & 0.08 & 0.09 & 0.05 & 0.55 & 0.07 & 0.72 & 1.24 & 22.35 & 7.67 \\
\hline Wilson Lake & РЕTM & 0.18 & 0.08 & 0.09 & 0.05 & 0.54 & 0.07 & 0.18 & 0.08 & 0.09 & 0.05 & 0.54 & 0.07 & 0.73 & 1.25 & 21.89 & 7.80 \\
\hline Wilson Lake & РЕТT & 0.21 & 0.08 & 0.09 & 0.04 & 0.53 & 0.05 & 0.21 & 0.08 & 0.09 & 0.04 & 0.53 & 0.05 & 0.70 & 1.33 & 22.19 & 7.91 \\
\hline 1218 & 33.07 & 49454 & 9302 & 7179 & 1405 & 60702 & 3285 & 0.38 & 0.07 & 0.05 & 0.01 & 0.46 & 0.03 & 0.56 & 0.26 & 19.14 & 3.50 \\
\hline 1218 & 33.26 & 31907 & 6171 & 4772 & 847 & 41242 & 2289 & 0.37 & 0.07 & 0.05 & 0.01 & 0.47 & 0.03 & 0.56 & 0.22 & 19.22 & 3.42 \\
\hline 998 & 33.229 & 47654 & 3212 & 5289 & 580 & 14660 & 2750 & 0.64 & 0.04 & 0.07 & 0.01 & 0.20 & 0.04 & 0.73 & 1.38 & 17.13 & 7.89 \\
\hline 998 & 33.322 & 338527 & 18728 & 29979 & 3056 & 110680 & 17159 & 0.65 & 0.04 & 0.06 & 0.01 & 0.21 & 0.03 & 0.73 & 1.11 & 12.72 & 7.07 \\
\hline 998 & 33.369 & 213019 & 16778 & 29858 & 2971 & 105871 & 21685 & 0.55 & 0.04 & 0.08 & 0.01 & 0.27 & 0.06 & 0.76 & 1.40 & 17.34 & 7.61 \\
\hline $\begin{array}{l}998 \\
998\end{array}$ & $\begin{array}{l}33.463 \\
33.510\end{array}$ & $\begin{array}{r}31199 \\
415782\end{array}$ & $\begin{array}{r}3129 \\
39363\end{array}$ & $\begin{array}{c}3872 \\
67061\end{array}$ & $\begin{array}{l}700 \\
7441\end{array}$ & $\begin{array}{l}13353 \\
1988170\end{array}$ & $\begin{array}{c}4880 \\
36185\end{array}$ & $\begin{array}{l}0.55 \\
0.54\end{array}$ & $\begin{array}{l}0.05 \\
0.05\end{array}$ & $\begin{array}{l}0.07 \\
0.09\end{array}$ & 0.01 & 0.23 & 0.09 & 0.75 & 1.65 & 15.35 & 8.37 \\
\hline $\begin{array}{l}998 \\
998\end{array}$ & $\begin{array}{l}33.510 \\
33.560\end{array}$ & $\begin{array}{l}415782 \\
353098\end{array}$ & $\begin{array}{l}39363 \\
32353\end{array}$ & $\begin{array}{l}67061 \\
66179\end{array}$ & $\begin{array}{l}7441 \\
6817\end{array}$ & $\begin{array}{l}198870 \\
226600\end{array}$ & $\begin{array}{l}36185 \\
43127\end{array}$ & $\begin{array}{l}0.54 \\
0.48\end{array}$ & $\begin{array}{l}0.05 \\
0.04\end{array}$ & $\begin{array}{l}0.09 \\
0.09\end{array}$ & $\begin{array}{l}0.01 \\
0.01\end{array}$ & $\begin{array}{l}0.26 \\
0.31\end{array}$ & $\begin{array}{l}0.05 \\
0.06\end{array}$ & $\begin{array}{l}0.74 \\
0.78\end{array}$ & $\begin{array}{l}1.39 \\
1.54\end{array}$ & $\begin{array}{l}20.54 \\
18.53\end{array}$ & 7.92 \\
\hline $\begin{array}{l}998 \\
998\end{array}$ & $\begin{array}{l}\begin{array}{r}33.560 \\
33.611\end{array} \\
\text { S }\end{array}$ & $\begin{array}{l}\begin{array}{r}3530988 \\
306680\end{array} \\
3\end{array}$ & $\begin{array}{l}\begin{array}{l}32353 \\
26715\end{array}\end{array}$ & $\begin{array}{l}66179 \\
53172\end{array}$ & $\begin{array}{l}6817 \\
5865\end{array}$ & $\begin{array}{l}226600 \\
183613\end{array}$ & $\begin{array}{l}312727 \\
35222\end{array}$ & $\begin{array}{l}0.48 \\
0.50\end{array}$ & $\begin{array}{l}0.04 \\
0.04\end{array}$ & $\begin{array}{l}0.09 \\
0.09\end{array}$ & $\begin{array}{l}0.01 \\
0.01\end{array}$ & $\begin{array}{l}0.31 \\
0.30\end{array}$ & $\begin{array}{l}0.06 \\
0.06\end{array}$ & $\begin{array}{l}0.78 \\
0.78\end{array}$ & $\begin{array}{l}1.54 \\
1.52\end{array}$ & $\begin{array}{l}18.83 \\
17.95\end{array}$ & $\begin{array}{l}7.95 \\
7.94\end{array}$ \\
\hline 998 & 33.669 & 80860 & 6164 & 13320 & 1056 & 34661 & $\begin{array}{l}35222 \\
6622\end{array}$ & 0.57 & $\begin{array}{l}0.04 \\
0.04\end{array}$ & 0.09 & 0.01 & 0.24 & 0.05 & 0.77 & $\begin{array}{l}1.52 \\
1.65\end{array}$ & $\begin{array}{l}18.95 \\
18.72\end{array}$ & $\begin{array}{l}7.94 \\
8.21\end{array}$ \\
\hline 998 & 33.726 & 30577 & 4652 & 2614 & 0 & 21599 & 1254 & 0.50 & 0.08 & 0.04 & 0.00 & 0.36 & 0.02 & 0.45 & 0.51 & 6.95 & $\begin{array}{l}0.11 \\
4.19\end{array}$ \\
\hline 998 & 33.783 & 45479 & 5783 & 3777 & 0 & 32377 & 1929 & 0.51 & 0.06 & 0.04 & 0.00 & 0.36 & 0.02 & 0.50 & 0.41 & 7.89 & 4.04 \\
\hline 998 & 33.886 & 283064 & 31190 & 41763 & 8536 & 141513 & 25343 & 0.53 & 0.06 & 0.08 & 0.02 & 0.27 & 0.05 & 0.71 & 1.30 & 11.91 & 7.94 \\
\hline 998 & 33.972 & 24963 & 3237 & 2208 & 200 & 16464 & 827 & 0.52 & 0.07 & 0.05 & 0.00 & 0.34 & 0.02 & 0.50 & 0.30 & 11.15 & 3.68 \\
\hline 998 & 34.147 & 42568 & 5626 & 3725 & 723 & 28941 & 1370 & 0.51 & 0.07 & 0.04 & 0.01 & 0.35 & 0.02 & 0.51 & 0.39 & 17.15 & 3.85 \\
\hline 998 & 34.235 & 49602 & 6467 & 5441 & 400 & 32704 & 2099 & 0.51 & 0.07 & 0.06 & 0.00 & 0.34 & 0.02 & 0.55 & 0.47 & 14.46 & 4.12 \\
\hline 998 & 34.411 & 18719 & 1425 & 2264 & 0 & 7910 & 1219 & 0.59 & 0.05 & 0.07 & 0.00 & 0.25 & 0.04 & 0.71 & 1.14 & 9.94 & 7.36 \\
\hline 803 & 28.987 & 412013 & 56372 & 42951 & 3996 & 98633 & 15077 & 0.65 & 0.09 & 0.07 & 0.01 & 0.16 & 0.02 & 0.52 & 0.93 & 11.80 & 6.35 \\
\hline 803 & 30.416 & 476338 & 64884 & 83359 & 11419 & 436176 & 73181 & 0.42 & 0.06 & 0.07 & 0.01 & 0.38 & 0.06 & 0.72 & 0.94 & 22.54 & 5.96 \\
\hline 803 & 33.241 & 29592 & 7076 & 7784 & 580 & 22786 & 3736 & 0.41 & 0.10 & 0.11 & 0.01 & 0.32 & 0.05 & 0.63 & 1.14 & 23.98 & 6.96 \\
\hline 803 & $\begin{array}{l}33.321 \\
\end{array}$ & 380168 & 145607 & 113055 & 12702 & $\begin{array}{l}488966 \\
2\end{array}$ & 64354 & 0.32 & 0.12 & 0.09 & 0.01 & 0.41 & 0.05 & 0.57 & 1.19 & 19.41 & 7.28 \\
\hline 803 & $\begin{array}{r}33.355 \\
33.395\end{array}$ & $\begin{array}{l}34489 \\
44019\end{array}$ & $\begin{array}{l}12575 \\
18782\end{array}$ & $\begin{array}{l}7360 \\
12410\end{array}$ & 860 & $\begin{array}{l}25325 \\
33940\end{array}$ & $\begin{array}{l}2711 \\
4744\end{array}$ & $\begin{array}{l}0.41 \\
0.39\end{array}$ & $\begin{array}{l}0.15 \\
0.16\end{array}$ & 0.09 & 0.01 & 0.30 & 0.03 & 0.47 & 2.32 & $\begin{array}{l}15.49 \\
15\end{array}$ & 8.85 \\
\hline $\begin{array}{l}803 \\
803\end{array}$ & $\begin{array}{l}33.399 \\
33.600\end{array}$ & $\begin{array}{r}44018 \\
354394\end{array}$ & $\begin{array}{l}18782 \\
93071\end{array}$ & $\begin{array}{l}12410 \\
99677\end{array}$ & $\begin{array}{l}990 \\
7717\end{array}$ & $\begin{array}{r}329290 \\
312905\end{array}$ & $\begin{array}{l}4741 \\
41031\end{array}$ & $\begin{array}{l}0.39 \\
0.39\end{array}$ & $\begin{array}{l}0.16 \\
0.10\end{array}$ & $\begin{array}{l}0.11 \\
0.11\end{array}$ & $\begin{array}{l}0.01 \\
0.01\end{array}$ & $\begin{array}{l}0.29 \\
0.34\end{array}$ & $\begin{array}{l}0.04 \\
0.05\end{array}$ & $\begin{array}{l}0.49 \\
0.61\end{array}$ & $\begin{array}{l}2.79 \\
1.05\end{array}$ & $\begin{array}{l}15.43 \\
23.95\end{array}$ & 8.90 \\
\hline $\begin{array}{l}803 \\
803\end{array}$ & $\begin{array}{l}\begin{array}{r}33.600 \\
33.639\end{array}\end{array}$ & $\begin{array}{r}354394 \\
29288\end{array}$ & $\begin{array}{l}93071 \\
4691\end{array}$ & $\begin{array}{l}99677 \\
3739\end{array}$ & $\begin{array}{c}7717 \\
618\end{array}$ & $\begin{array}{l}3121205 \\
20812\end{array}$ & $\begin{array}{c}41031 \\
1665\end{array}$ & $\begin{array}{l}0.39 \\
0.48\end{array}$ & $\begin{array}{l}0.10 \\
0.08\end{array}$ & $\begin{array}{l}0.11 \\
0.06\end{array}$ & $\begin{array}{l}0.01 \\
0.01\end{array}$ & $\begin{array}{l}0.34 \\
0.34\end{array}$ & $\begin{array}{l}0.05 \\
0.03\end{array}$ & $\begin{array}{l}0.61 \\
0.56\end{array}$ & $\begin{array}{l}1.05 \\
0.61\end{array}$ & $\begin{array}{l}23.95 \\
19.85\end{array}$ & $\begin{array}{l}6.73 \\
4.55\end{array}$ \\
\hline 803 & 33.657 & 122414 & 29866 & 30808 & 2616 & 94218 & 13382 & 0.42 & $\begin{array}{l}0.08 \\
0.10\end{array}$ & 0.11 & 0.01 & $\begin{array}{l}0.34 \\
0.32\end{array}$ & 0.05 & 0.61 & $\begin{array}{l}0.17 \\
1.17\end{array}$ & $\begin{array}{l}19.85 \\
23.46\end{array}$ & $\begin{array}{l}4.53 \\
6.85\end{array}$ \\
\hline 803 & & 73187 & 16781 & 21786 & 2000 & 58675 & 9407 & 0.40 & 0.09 & 0.12 & 0.01 & 0.32 & 0.05 & 0.66 & 1.18 & 25.00 & 7.25 \\
\hline 803 & & 107996 & 19762 & 22052 & 2319 & 84829 & 11009 & 0.44 & 0.08 & 0.09 & 0.01 & 0.34 & 0.04 & 0.64 & 0.75 & 26.19 & 5.63 \\
\hline 803 & & 211755 & $\begin{array}{l}48250 \\
77505\end{array}$ & $\begin{array}{r}65701 \\
\end{array}$ & $\begin{array}{l}6450 \\
\end{array}$ & 181373 & 35687 & 0.39 & 0.09 & 0.12 & 0.01 & 0.33 & 0.06 & 0.69 & 1.17 & $\begin{array}{r}25.53 \\
25.53\end{array}$ & 7.11 \\
\hline 803 & & 245491 & $\begin{array}{c}70511 \\
\end{array}$ & 105569 & $\begin{array}{c}9823 \\
16213\end{array}$ & $\begin{array}{l}309589 \\
520870\end{array}$ & $\begin{array}{l}62635 \\
\end{array}$ & 0.31 & 0.09 & 0.13 & 0.01 & 0.39 & 0.08 & 0.72 & 1.15 & $\begin{array}{l}25.12 \\
26.12\end{array}$ & 6.71 \\
\hline $\begin{array}{l}803 \\
803\end{array}$ & & $\begin{array}{l}4995359 \\
402542\end{array}$ & $\begin{array}{l}118841 \\
107438\end{array}$ & $\begin{array}{l}170631 \\
15701\end{array}$ & $\begin{array}{l}16215 \\
15594\end{array}$ & $\begin{array}{l}5280077 \\
503329\end{array}$ & $\begin{array}{l}95625 \\
80177\end{array}$ & $\begin{array}{l}0.35 \\
0.32\end{array}$ & $\begin{array}{l}0.08 \\
0.08\end{array}$ & $\begin{array}{l}0.12 \\
0.12\end{array}$ & $\begin{array}{l}0.01 \\
0.01\end{array}$ & $\begin{array}{l}0.37 \\
0.40\end{array}$ & $\begin{array}{l}0.07 \\
0.06\end{array}$ & $\begin{array}{l}0.70 \\
0.70\end{array}$ & 0.95 & $\begin{array}{l}26.87 \\
27.87\end{array}$ & 6.32 \\
\hline $\begin{array}{l}803 \\
628\end{array}$ & 32.118 & $\begin{array}{l}402542 \\
274282\end{array}$ & $\begin{array}{l}107738 \\
46758\end{array}$ & $\begin{array}{l}157601 \\
76973\end{array}$ & $\begin{array}{l}155594 \\
29566\end{array}$ & $\begin{array}{l}5032329 \\
403070\end{array}$ & $\begin{array}{l}80177 \\
52021\end{array}$ & $\begin{array}{l}0.32 \\
0.31\end{array}$ & $\begin{array}{l}0.08 \\
0.05\end{array}$ & $\begin{array}{l}0.12 \\
0.09\end{array}$ & $\begin{array}{l}0.01 \\
0.03\end{array}$ & $\begin{array}{l}0.40 \\
0.46\end{array}$ & $\begin{array}{l}0.06 \\
0.06\end{array}$ & $\begin{array}{l}0.70 \\
0.77\end{array}$ & $\begin{array}{l}0.98 \\
1.76\end{array}$ & $\begin{array}{l}27.22 \\
17.74\end{array}$ & $\begin{array}{l}5.96 \\
8.74\end{array}$ \\
\hline 628 & 32.226 & $\begin{array}{l}17482 \\
101251\end{array}$ & $\begin{array}{l}46 / 58 \\
14688\end{array}$ & $\begin{array}{l}11979 \\
1197\end{array}$ & $\begin{array}{l}25506 \\
1762\end{array}$ & 76007 & $\begin{array}{c}25021 \\
5552\end{array}$ & 0.48 & $\begin{array}{l}0.05 \\
0.07\end{array}$ & $\begin{array}{l}0.09 \\
0.06\end{array}$ & $\begin{array}{l}0.03 \\
0.01\end{array}$ & $\begin{array}{l}0.46 \\
0.36\end{array}$ & $\begin{array}{l}0.06 \\
0.03\end{array}$ & 0.57 & $\begin{array}{l}1.16 \\
0.37\end{array}$ & $\begin{array}{l}11.14 \\
20.39\end{array}$ & $\begin{array}{l}8.14 \\
3.91\end{array}$ \\
\hline 628 & 32.284 & 120713 & 27377 & 64538 & 8514 & 206080 & 41183 & 0.26 & 0.06 & 0.14 & 0.02 & 0.44 & 0.09 & 0.81 & 1.20 & 23.51 & 7.28 \\
\hline 628 & 32.703 & 309769 & 44728 & 55885 & 7965 & 267192 & 33686 & 0.43 & 0.06 & 0.08 & 0.01 & 0.37 & 0.05 & 0.69 & 0.92 & 22.41 & 5.82 \\
\hline 628 & 32.757 & 54781 & 9394 & 19258 & 2142 & 56056 & 10363 & 0.36 & 0.06 & 0.13 & 0.01 & 0.37 & 0.07 & 0.77 & 1.31 & 25.00 & 7.41 \\
\hline 628 & 32.807 & $\begin{array}{l}73706 \\
775\end{array}$ & 12077 & 17571 & 2070 & 67898 & 9011 & 0.40 & 0.07 & 0.10 & 0.01 & 0.37 & 0.05 & 0.70 & 1.00 & 25.19 & 6.19 \\
\hline $\begin{array}{l}628 \\
628\end{array}$ & $\begin{array}{r}32.861 \\
32.905\end{array}$ & $\begin{array}{c}75501 \\
241995\end{array}$ & $\begin{array}{l}11513 \\
40066\end{array}$ & $\begin{array}{c}9587 \\
4241\end{array}$ & $\begin{array}{c}1282 \\
12499\end{array}$ & $\begin{array}{c}66007 \\
2491118\end{array}$ & $\begin{array}{c}3983 \\
2244\end{array}$ & $\begin{array}{l}0.45 \\
0.40\end{array}$ & $\begin{array}{l}0.07 \\
0.07\end{array}$ & $\begin{array}{l}0.06 \\
0.07\end{array}$ & $\begin{array}{l}0.01 \\
0.02\end{array}$ & $\begin{array}{l}0.39 \\
0.41\end{array}$ & $\begin{array}{l}0.02 \\
0.04\end{array}$ & $\begin{array}{l}0.56 \\
0.66\end{array}$ & $\begin{array}{l}0.29 \\
0.77\end{array}$ & $\begin{array}{l}19.99 \\
20.44\end{array}$ & $\begin{array}{l}3.71 \\
648\end{array}$ \\
\hline $\begin{array}{l}628 \\
628\end{array}$ & $\begin{array}{l}32.905 \\
33.332\end{array}$ & $\begin{array}{r}241995 \\
73193\end{array}$ & $\begin{array}{l}40066 \\
13378\end{array}$ & $\begin{array}{l}42415 \\
31635\end{array}$ & $\begin{array}{l}15499 \\
3656\end{array}$ & $\begin{array}{l}\begin{array}{l}249918 \\
104341\end{array}\end{array}$ & $\begin{array}{l}22447 \\
19616\end{array}$ & $\begin{array}{l}0.40 \\
0.30\end{array}$ & $\begin{array}{l}0.07 \\
0.05\end{array}$ & $\begin{array}{l}0.07 \\
0.13\end{array}$ & $\begin{array}{l}0.02 \\
0.01\end{array}$ & $\begin{array}{l}0.41 \\
0.42\end{array}$ & $\begin{array}{l}0.04 \\
0.08\end{array}$ & $\begin{array}{l}0.66 \\
0.80 \\
0.80\end{array}$ & $\begin{array}{l}0.77 \\
1.20\end{array}$ & $\begin{array}{l}20.44 \\
25.96\end{array}$ & $\begin{array}{l}6.48 \\
7.12\end{array}$ \\
\hline $\begin{array}{l}628 \\
628\end{array}$ & $\begin{array}{l}33.392 \\
33.446\end{array}$ & $\begin{array}{r}319193 \\
131321\end{array}$ & $\begin{array}{l}13378 \\
24138\end{array}$ & $\begin{array}{l}33635 \\
55126\end{array}$ & $\begin{array}{l}3656 \\
8210\end{array}$ & $\begin{array}{l}104441 \\
202149\end{array}$ & $\begin{array}{l}19616 \\
37084\end{array}$ & $\begin{array}{l}0.30 \\
0.29\end{array}$ & $\begin{array}{l}0.05 \\
0.05\end{array}$ & $\begin{array}{l}0.13 \\
0.12\end{array}$ & $\begin{array}{l}0.01 \\
0.02\end{array}$ & $\begin{array}{l}0.42 \\
0.44\end{array}$ & $\begin{array}{l}0.08 \\
0.08\end{array}$ & $\begin{array}{l}0.80 \\
0.81\end{array}$ & $\begin{array}{l}1.20 \\
1.14\end{array}$ & $\begin{array}{l}25.96 \\
24.79\end{array}$ & $\begin{array}{l}7.12 \\
7.05\end{array}$ \\
\hline 628 & 33.500 & 235860 & 39449 & 79728 & 10553 & 306535 & 51676 & 0.33 & 0.05 & 0.11 & 0.01 & 0.42 & 0.07 & 0.78 & 1.02 & 27.45 & 6.39 \\
\hline 628 & 33.554 & 207632 & 38418 & 99025 & 13433 & 390490 & 74224 & 0.25 & 0.05 & 0.12 & 0.02 & 0.47 & 0.09 & 0.83 & 1.15 & 25.19 & 7.04 \\
\hline 628 & 33.609 & 130100 & 21365 & 23532 & 3859 & 134179 & 13060 & 0.40 & 0.07 & 0.07 & 0.01 & 0.41 & 0.04 & 0.65 & 0.64 & 22.75 & 4.72 \\
\hline 628 & 33.645 & 97250 & 15864 & 38084 & 5860 & 149686 & 28336 & 0.29 & 0.05 & 0.11 & 0.02 & 0.45 & 0.08 & 0.82 & 1.21 & 24.75 & 7.22 \\
\hline 628 & & $\begin{array}{l}33923 \\
1\end{array}$ & $\begin{array}{l}6472 \\
0\end{array}$ & $\begin{array}{l}146999 \\
54545\end{array}$ & $\begin{array}{l}2209 \\
90707\end{array}$ & $\begin{array}{r}53938 \\
225532\end{array}$ & $\begin{array}{l}10263 \\
-3\end{array}$ & 0.28 & 0.05 & 0.12 & 0.02 & 0.44 & 0.08 & 0.81 & 1.11 & 24.56 & 05 \\
\hline & & 124219 & 20750 & 52454 & & 225531 & 39997 & & & & 0.02 & & & & & 23.16 & 7.22 \\
\hline
\end{tabular}


Cretaceous dataset for OPTiMAL

$\begin{array}{llllllllllllll}\text { AGE (GTS 2012) } & 1302 & 1300 & 1298 & 1296 & 1292 & 1292 & \text { Fr. } 1302 & \text { Fr. } 1300 & \text { Fr. } 1298 & \text { Fr.1296 } & \text { Fr.1292 } & \text { Fr.1292 } & \text { TEX }\end{array}$

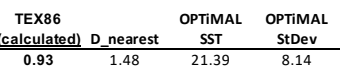

\begin{tabular}{|c|c|c|c|c|c|c|c|c|c|c|c|c|c|}
\hline ODP 1049 & 112.57 & $\begin{array}{l}\text { GDGGi0 } \\
446000\end{array}$ & $\frac{6 \text { G6GI1 }}{118000}$ & $\begin{array}{l}G D G 12 \\
726000\end{array}$ & $\begin{array}{l}60 D G 13 \\
95300\end{array}$ & $\begin{array}{l}\text { CREN } \\
5011000\end{array}$ & $\begin{array}{l}\text { CREN } \\
7880000\end{array}$ & 0.062080 & 0.016425 & 0.101054 & 0.013265 & 0.697493 & 0.109684 \\
\hline & & & & & & & & & & & & & \\
\hline & 131.90 & 5787 & 100790 & 303؛ & 108330 & 2706610 & 27683 & 0.042432 & $0.0257 \mathrm{~g}$ & 077 & 0.02772 & 9277 & .133521 \\
\hline (2) 60 & 132.20 & 312 & 105467 & 28516 & & 396660 & 76112 & 0.051972 & 0.0251 & 0.080 & 0.02782 & $0.6 / 58$ & 134252 \\
\hline 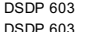 & $\begin{array}{l}132.46 \\
13253\end{array}$ & 440 & $\begin{array}{l}92422 \\
\end{array}$ & 297226 & 130920 & 627850 & 13216 & 0.05004 & 0.02428 & 0.078101 & T. & 0.690511 & 0.134856 \\
\hline & $\begin{array}{l}1232.53 \\
1323\end{array}$ & 11117 & $\begin{array}{r}68499 \\
67909\end{array}$ & 2000851 & 8390 & & 0 & & & 0.069 & .029 & 0.699975 & 0.138631 \\
\hline 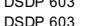 & $\begin{array}{l}133.03 \\
{ }_{13}^{3} 03\end{array}$ & $\begin{array}{l}312780 \\
48072\end{array}$ & $\begin{array}{l}272041 \\
2861\end{array}$ & $\begin{array}{l}4833747 \\
77251\end{array}$ & $\begin{array}{l}2170017 \\
{ }_{20375}\end{array}$ & 556643900 & $\begin{array}{l}18068560 \\
158836\end{array}$ & 0.038969 & 0.027958 & 0.061741 & 0.027510 & $\begin{array}{l}0.7110185 \\
0.72446\end{array}$ & 0.136878 \\
\hline DSDP 603 & 133.16 & $\begin{array}{l}40012 \\
126022\end{array}$ & $\begin{array}{l}80034 \\
80304\end{array}$ & 247989 & $\begin{array}{r}121999 \\
121993\end{array}$ & $\begin{array}{l}2771444 \\
27740\end{array}$ & $\begin{array}{l}\text { rordso } \\
577954\end{array}$ & $\begin{array}{l}0.03532151 \\
0.032\end{array}$ & 0.020487 & $\begin{array}{l}0.063267 \\
0.06\end{array}$ & $\begin{array}{l}0.031123 \\
0.03\end{array}$ & 0.707055 & 0.145917 \\
\hline DSDP 603 & 133.42 & 35479 & 21482 & 62823 & 31801 & 677610 & 132721 & 0.036884 & 0.022333 & 0.065310 & 0.033060 & 0.704438 & 0.137976 \\
\hline DP 603 & $\begin{array}{l}133.55 \\
134.22\end{array}$ & $\begin{array}{l}87178 \\
83648\end{array}$ & $\begin{array}{r}57698 \\
{ }_{5}^{5} 6503\end{array}$ & $\begin{array}{l}143778 \\
159003\end{array}$ & $\begin{array}{l}62685 \\
70355\end{array}$ & 1505120 & $\begin{array}{r}296720 \\
325800\end{array}$ & 0.040488 & 0.026797 & 0.066775 & 0.029113 & 0.699022 & 0.137806 \\
\hline $\begin{array}{l}\text { DP 603 } \\
\text { DP } 603\end{array}$ & $\begin{array}{l}134.22 \\
13547\end{array}$ & $\begin{array}{l}83644 \\
28429\end{array}$ & $\begin{array}{l}52603 \\
15942\end{array}$ & $\begin{array}{l}159003 \\
40258\end{array}$ & & $\begin{array}{l}1658240 \\
499800\end{array}$ & $\begin{array}{l}328509 \\
909997\end{array}$ & $\begin{array}{l}0.035557 \\
0.04437\end{array}$ & $\begin{array}{l}0.022362 \\
0.02488\end{array}$ & $\begin{array}{l}0.067607 \\
0.068240\end{array}$ & $\begin{array}{l}0.029904 \\
0.023744\end{array}$ & $\begin{array}{l}0.704921 \\
0702111\end{array}$ & $\begin{array}{l}0.139650 \\
0.142040\end{array}$ \\
\hline 6003 & $\begin{array}{l}735.47 \\
135.62\end{array}$ & $\begin{array}{l}20429 \\
20250\end{array}$ & $\begin{array}{l}109424 \\
12111\end{array}$ & $\begin{array}{l}40050 \\
35974\end{array}$ & $\begin{array}{l}15214 \\
17577\end{array}$ & $\begin{array}{l}449802 \\
425736\end{array}$ & 82585 & $\begin{array}{l}0.044376 \\
0.034078\end{array}$ & $\begin{array}{l}0.0248684 \\
0.02031\end{array}$ & 0 & 0.029579 & 0.716446 & $\begin{array}{l}0.142040 \\
0.138977\end{array}$ \\
\hline 603 & 135.90 & 24351 & 15844 & 35515 & 21309 & 480880 & 88566 & 0.036538 & 0.023773 & 0.053289 & 0.031973 & 0.721538 & 0.132889 \\
\hline & 136.18 & 30025 & 23069 & 49333 & 27022 & 512579 & 96082 & 0.040678 & 0.031254 & 0.066837 & 0.036610 & 0.694448 & 0.130173 \\
\hline $\begin{array}{l}603 \\
603 \\
603\end{array}$ & $\begin{array}{l}\begin{array}{l}136.30 \\
16.55\end{array}\end{array}$ & $\begin{array}{l}19268 \\
25844\end{array}$ & $\begin{array}{l}12874 \\
16120\end{array}$ & $\begin{array}{l}28719 \\
27836\end{array}$ & $\begin{array}{l}21050 \\
18062\end{array}$ & $\begin{array}{l}383234 \\
394334\end{array}$ & $\begin{array}{l}71733 \\
61937\end{array}$ & $\begin{array}{l}0.035889 \\
0.5518\end{array}$ & $\begin{array}{l}0.023979 \\
0\end{array}$ & 0.053493 & 0.039208 & $\begin{array}{l}0.713820 \\
077\end{array}$ & 0.133611 \\
\hline & 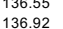 & & & & $\begin{array}{l}18062 \\
29594\end{array}$ & $\begin{array}{l}349337 \\
699404\end{array}$ & $\begin{array}{l}613727 \\
126414\end{array}$ & $\begin{array}{l}0.0518344 \\
0.037488\end{array}$ & $\begin{array}{l}0.0332332 \\
0.022152\end{array}$ & $\begin{array}{l}0.0558332 \\
0.054616\end{array}$ & 0.036228 & $\begin{array}{l}0.1006 / 8 \\
0.718332\end{array}$ & $\begin{array}{l}0.1233096 \\
0.135654\end{array}$ \\
\hline P 603 & 136.97 & $\begin{array}{l}32935 \\
32904\end{array}$ & $\begin{array}{l}206043 \\
22742\end{array}$ & $\begin{array}{l}58096 \\
45981\end{array}$ & $\begin{array}{l}29594 \\
24883\end{array}$ & $\begin{array}{l}669404 \\
584841\end{array}$ & $\begin{array}{l}126414 \\
107838\end{array}$ & $\begin{array}{l}0.033 / 488 \\
0.040167\end{array}$ & $\begin{array}{l}0.022152 \\
0.02772\end{array}$ & $\begin{array}{l}0.0564616 \\
0.056130\end{array}$ & $\begin{array}{l}0.0371 / 1 \\
0.030375\end{array}$ & $\begin{array}{l}0.183332 \\
0.71327\end{array}$ & $\begin{array}{l}0.135654 \\
0.131640\end{array}$ \\
\hline & 94.17 & 242000 & 44900 & 25100 & 63600 & 2270000 & 377000 & 0.080064 & 0.014855 & 0.008304 & 0.021041 & 0.751009 & 0.124727 \\
\hline & $\begin{array}{l}94.21 \\
94.22\end{array}$ & 395000 & $\begin{array}{l}67200 \\
54650\end{array}$ & $\begin{array}{l}65400 \\
50700\end{array}$ & 39200 & 2840000 & 484000 & 0.101522 & 0.017272 & 0.016809 & 0.010075 & 0.729927 & 0.124396 \\
\hline & $\begin{array}{l}94.22 \\
94.25\end{array}$ & $\begin{array}{r}2233000 \\
1230000\end{array}$ & $\begin{array}{r}456000 \\
200000\end{array}$ & $\begin{array}{l}50700 \\
364000\end{array}$ & $\begin{array}{r}31400 \\
10000\end{array}$ & $\begin{array}{l}1450000 \\
6190000\end{array}$ & $\begin{array}{r}286000 \\
19700000\end{array}$ & 0.106867 & $\begin{array}{r}0.021853 \\
0.02184\end{array}$ & $\begin{array}{l}0.024297 \\
0.03976\end{array}$ & 0.015048 & $\begin{array}{l}0.694877 \\
0\end{array}$ & 0.137059 \\
\hline 367 & $\begin{array}{l}94.25 \\
94.29\end{array}$ & $\begin{array}{l}\begin{array}{l}12300000 \\
148000\end{array}\end{array}$ & $\begin{array}{l}\begin{array}{l}2000000 \\
24000\end{array} \\
2\end{array}$ & $\begin{array}{l}6640000 \\
42000\end{array}$ & $\begin{array}{l}\begin{array}{l}1000000 \\
50000\end{array} \\
5\end{array}$ & 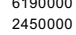 & $\begin{array}{l}170700000 \\
3860000\end{array}$ & $\begin{array}{l}0.134367 \\
0.047742\end{array}$ & $\begin{array}{l}0.021848 \\
0.007742\end{array}$ & $\begin{array}{l}0.033964 \\
0.013548\end{array}$ & $\begin{array}{l}0.01092424 \\
0.016129\end{array}$ & $\begin{array}{l}0.6772027 \\
0.790323\end{array}$ & $\begin{array}{l}0.1116889 \\
0.124516\end{array}$ \\
\hline P 367 & 94.31 & $\begin{array}{l}1670000 \\
\end{array}$ & $\begin{array}{l}26400 \\
26400\end{array}$ & 98000 & 46500 & 2430000 & $\begin{array}{l}400000 \\
434000\end{array}$ & $\begin{array}{l}0.052157 \\
0.052\end{array}$ & 0.008245 & 0.030607 & 0.014523 & 0.758924 & 0.135545 \\
\hline & 94.33 & 214000 & 55300 & 106000 & 75800 & 3020000 & 454000 & 0.054521 & 0.014089 & 0.027006 & 0.019312 & 0.769407 & 0.115666 \\
\hline $\begin{array}{l}\text { P } 367 \\
\text { P }\end{array}$ & $\begin{array}{l}94.38 \\
94.40\end{array}$ & $\begin{array}{l}236000 \\
569000\end{array}$ & $\begin{array}{r}28700 \\
197000\end{array}$ & $\begin{array}{l}52600 \\
338000\end{array}$ & $\begin{array}{r}52100 \\
246000\end{array}$ & 2080000 & $\begin{array}{r}322000 \\
12000\end{array}$ & 0.085156 & 0.010356 & 0.018980 & 0.018799 & 0.750523 & 0.116187 \\
\hline & $\begin{array}{l}94.40 \\
94.52\end{array}$ & $\begin{array}{l}569000 \\
180000\end{array}$ & $\begin{array}{l}1919000 \\
112000\end{array}$ & $\begin{array}{l}3380000 \\
115000\end{array}$ & $\begin{array}{l}246000 \\
87400\end{array}$ & $\begin{array}{r}66900000 \\
4050000\end{array}$ & $\begin{array}{l}10300000 \\
6960000\end{array}$ & $\begin{array}{l}0.062776 \\
0.034349\end{array}$ & $\begin{array}{l}0.0211072 \\
0.021372\end{array}$ & $\begin{array}{l}0.037290 \\
0.02195\end{array}$ & $\begin{array}{l}0.027140 \\
0.016678\end{array}$ & $\begin{array}{l}0.738085 \\
0.772882\end{array}$ & 0.113636 \\
\hline 367 & 94.53 & 233000 & 166000 & 250000 & 156000 & $\begin{array}{l}\text { } 4050000 \\
7320000\end{array}$ & $\begin{array}{l}69960000 \\
1050000\end{array}$ & $\begin{array}{l}0.0334349 \\
0.025395\end{array}$ & $\begin{array}{l}0.02173 / 2 \\
0.018093\end{array}$ & $\begin{array}{l}0.0221945 \\
0.027248\end{array}$ & 0.017003 & 0 & $\begin{array}{l}0.138214 \\
0.11441\end{array}$ \\
\hline & 94.56 & 199000 & 66000 & 194000 & 107000 & 2760000 & 526000 & 0.051661 & 0.017134 & 0.050363 & 0.027778 & 0.716511 & 0.136552 \\
\hline 367 & 94.61 & $\begin{array}{l}132000 \\
\end{array}$ & 51900 & 89400 & 18700 & 3160000 & 391000 & 0.034348 & 0.013505 & 0.023263 & 0.004866 & 0.822274 & 0.101743 \\
\hline $\begin{array}{l}367 \\
467\end{array}$ & $\begin{array}{l}94.68 \\
12428\end{array}$ & $\begin{array}{c}2540000 \\
58900\end{array}$ & $\begin{array}{l}92000 \\
28870\end{array}$ & $\begin{array}{l}261000 \\
48000\end{array}$ & $\begin{array}{l}151000 \\
16100\end{array}$ & $\begin{array}{r}56900000 \\
35600\end{array}$ & $\begin{array}{l}11800000 \\
558800\end{array}$ & 0.033298 & $\begin{array}{l}0.012061 \\
0.05140\end{array}$ & $\begin{array}{l}0.034216 \\
0.073079\end{array}$ & 0.019795 & $\begin{array}{l}0.745936 \\
0.63765\end{array}$ & 0.154693 \\
\hline P 403 & $\begin{array}{l}174.26 \\
124.32\end{array}$ & 00 & $\begin{array}{l}887800 \\
5820\end{array}$ & $\begin{array}{l}48000 \\
4620\end{array}$ & $\begin{array}{l}101700 \\
4760\end{array}$ & $\begin{array}{l}35600 \\
5640\end{array}$ & $\begin{array}{l}158800 \\
16800\end{array}$ & $\begin{array}{l}0.105499 \\
0.19196\end{array}$ & 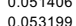 & 0.042230 & $\begin{array}{l}0.043838 \\
0.045\end{array}$ & $\begin{array}{r}0.0353503 \\
0.51539\end{array}$ & $\begin{array}{l}0.103329 \\
0.153565\end{array}$ \\
\hline DSDP 463 & 124.53 & 16400 & 5800 & 7300 & 2940 & 46700 & $\begin{array}{l}108000 \\
11900\end{array}$ & 0.180141 & 0.063708 & 0.080185 & 0.032293 & 0.512961 & 0.130712 \\
\hline & 124.67 & & 22200 & 55800 & 12900 & 40 & 51100 & & & 0.096141 & & 0.699518 & 0.088043 \\
\hline P 463 & $\begin{array}{l}124.91 \\
130.01\end{array}$ & $\begin{array}{l}17200 \\
846515\end{array}$ & $\begin{array}{c}8530 \\
500681\end{array}$ & $\begin{array}{c}5890 \\
1767720\end{array}$ & $\begin{array}{c}1000 \\
500009\end{array}$ & $\begin{array}{l}65000 \\
0\end{array}$ & $\begin{array}{r}25300 \\
2105780\end{array}$ & 0.139928 & 0.069395 & 0.047917 & 0.008135 & 0.528799 & 0.205825 \\
\hline & $\begin{array}{l}\begin{array}{l}130.01 \\
10.01\end{array} \\
10.13\end{array}$ & & $\begin{array}{l}500681 \\
935218\end{array}$ & $\begin{array}{l}17661720 \\
349770\end{array}$ & $\begin{array}{l}500009 \\
1012095\end{array}$ & $\begin{array}{l}153318200 \\
3078800\end{array}$ & $\begin{array}{l}3105780 \\
6371840\end{array}$ & $\begin{array}{l}0.038420 \\
0.04030\end{array}$ & $\begin{array}{l}0.022724 \\
0.021027\end{array}$ & $\begin{array}{l}0.079959 \\
0.076842\end{array}$ & $\begin{array}{l}0.022694 \\
0.022774\end{array}$ & $\begin{array}{l}0.695242 \\
0.699259\end{array}$ & 0.140961 \\
\hline P 534 & $\begin{array}{l}130.93 \\
130.96\end{array}$ & $\begin{array}{l}1780800 \\
1386550\end{array}$ & $\begin{array}{l}935218 \\
774860\end{array}$ & $\begin{array}{r}34997700 \\
2706840\end{array}$ & $\begin{array}{l}1 \begin{array}{l}1212550 \\
767937\end{array} \\
7\end{array}$ & $\begin{array}{r}30878000 \\
24910700\end{array}$ & $\begin{array}{l}63718480 \\
48949910\end{array}$ & $\begin{array}{l}0.040038 \\
0.039122\end{array}$ & $\begin{array}{l}0.021027 \\
0.02183\end{array}$ & $\begin{array}{l}0.078642 \\
0.076374\end{array}$ & $\begin{array}{l}0.022168 \\
0.02166\end{array}$ & $\begin{array}{l}0.699259 \\
0.702862\end{array}$ & $\begin{array}{l}0.143260 \\
0.138111\end{array}$ \\
\hline & 131.19 & 474030 & 257915 & 924411 & 274295 & 8031170 & 1709550 & 0.040615 & 0.022098 & 0.079204 & 0.023502 & 0.688108 & 0.146474 \\
\hline 3 & $\begin{array}{l}131.94 \\
13227\end{array}$ & $\begin{array}{l}282054 \\
207352\end{array}$ & $\begin{array}{l}170959 \\
1377659\end{array}$ & $\begin{array}{r}555242 \\
471196\end{array}$ & 179401 & 5407060 & 1109240 & 0.036612 & 0.022191 & 0.072072 & 0.023287 & 0.701855 & 0.143983 \\
\hline 4 & $\begin{array}{l}132.27 \\
13222\end{array}$ & & & $\begin{array}{l}477186 \\
654906\end{array}$ & & & & 00625 & 0.018292 & 0.063357 & 0.023513 & 0.717665 & 0.144332 \\
\hline $\begin{array}{l}\text { P 534 } \\
\text { P } 344\end{array}$ & 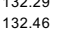 & $\begin{array}{l}46675 \\
206396\end{array}$ & $\begin{array}{l}22125 \\
92395\end{array}$ & $\begin{array}{c}644922 \\
280987\end{array}$ & $\begin{array}{l}20550 \\
104947\end{array}$ & $\begin{array}{r}6888993 \\
2803530\end{array}$ & $\begin{array}{l}154852 \\
600750\end{array}$ & $\begin{array}{l}0.046259 \\
0.050476\end{array}$ & $\begin{array}{l}0.0222165 \\
0.025259\end{array}$ & 0.060789 & 0 & $\begin{array}{l}0.693176 \\
0.6852\end{array}$ & $\begin{array}{l}0.155133 \\
0.146918\end{array}$ \\
\hline & 132.52 & 67927 & 27882 & 83663 & 31713 & $\begin{array}{l}880035300 \\
103840\end{array}$ & 203851 & 0.046734 & 0.019183 & 0.057560 & 0 & 0.714455 & $\begin{array}{l}0.146518 \\
0.140250\end{array}$ \\
\hline P 534 & 132.59 & 86357 & 37582 & 93776 & 30062 & 1072270 & 208885 & 0.056482 & 0.024581 & 0.061334 & 0.019662 & 0.701320 & 0.136622 \\
\hline & $\begin{array}{l}132.80 \\
13.202\end{array}$ & $\begin{array}{l}188983 \\
31139\end{array}$ & 102627 & 337897 & 121432 & & 695108 & 0.039497 & 0.021449 & 0.070621 & & 0.697776 & 0.145278 \\
\hline 5534 & $\begin{array}{l}1332.27 \\
1324\end{array}$ & $\begin{array}{l}31639 \\
30149\end{array}$ & $\begin{array}{l}15840 \\
14886\end{array}$ & $\begin{array}{l}52304 \\
50790\end{array}$ & $\begin{array}{l}16826 \\
17377\end{array}$ & $\begin{array}{l}582154 \\
493848\end{array}$ & $\begin{array}{l}116457 \\
99996\end{array}$ & $\begin{array}{l}0.038810 \\
0.042642\end{array}$ & 0.019430 & 0.064159 & 0.020640 & 0.714107 & 0.142853 \\
\hline$P 534$ & 3.46 & $\begin{array}{l}302149 \\
192104\end{array}$ & $\begin{array}{c}14880 \\
112455\end{array}$ & 362444 & 125543 & $\begin{array}{l}49 \\
31:\end{array}$ & $\begin{array}{l}66391769 \\
66326\end{array}$ & $\begin{array}{l}0.042642 \\
0.041870\end{array}$ & $\begin{array}{l}0.024 \\
0.0241\end{array}$ & 0.078997 & $\begin{array}{l}0.0243736 \\
0.027\end{array}$ & 0.08862696 & $\begin{array}{l}0.1441403 \\
0.144564\end{array}$ \\
\hline 34 & .25 & 258577 & $\begin{array}{l}16245041 \\
1600\end{array}$ & $\begin{array}{l}5382474 \\
538779\end{array}$ & $\begin{array}{l}160274 \\
1602\end{array}$ & 64 & $\begin{array}{l}1288220 \\
12820\end{array}$ & 0.029320 & 0.018827 & 0.061092 & 0.018173 & 0.726515 & 0.146071 \\
\hline & . & 24878 & 12126 & 33498 & 10962 & 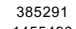 & 80864 & 0.045429 & 0.0 & 0.061170 & 018 & 0.703575 & 0.147665 \\
\hline P 5 P54 & $\begin{array}{l}134.57 \\
13257\end{array}$ & $\begin{array}{r}68605 \\
185464\end{array}$ & $\begin{array}{r}42246 \\
\end{array}$ & $\begin{array}{l}125595 \\
-320324\end{array}$ & $\begin{array}{r}41268 \\
109245\end{array}$ & $\begin{array}{l}1455490 \\
\quad 35205\end{array}$ & $\begin{array}{r}289521 \\
7205027\end{array}$ & 0.033917 & 0.020886 & 0.062092 & 0.020402 & 0.719569 & 0.143134 \\
\hline & & 185464 & $\begin{array}{l}102367 \\
573444\end{array}$ & $\begin{array}{l}3503034 \\
181007\end{array}$ & $\begin{array}{l}109345 \\
68338\end{array}$ & $\begin{array}{l}35266860 \\
2309690\end{array}$ & $\begin{array}{l}7366027 \\
446456\end{array}$ & $\begin{array}{l}0.037016 \\
0.043242\end{array}$ & 0.020437 & $\begin{array}{l}0.066921 \\
0.05652\end{array}$ & 0.021824 & $\begin{array}{l}0.7309808 \\
072140\end{array}$ & 0.146900 \\
\hline 34 & $\begin{array}{l}134.88 \\
134.88\end{array}$ & $\begin{array}{l}1388429 \\
57458\end{array}$ & $\begin{array}{l}5 / 3,344 \\
30030\end{array}$ & $\begin{array}{l}181007 \\
81505\end{array}$ & $\begin{array}{l}68 \\
29\end{array}$ & $\begin{array}{l}23006990 \\
1041400\end{array}$ & $\begin{array}{l}4446456 \\
235035\end{array}$ & $\begin{array}{l}0.043242 \\
0.038955\end{array}$ & $\begin{array}{l}0.01 / 913 \\
0.020399\end{array}$ & $\begin{array}{l}0.0565642 \\
0.055258\end{array}$ & $\begin{array}{l}0.02134 / 7 \\
0.020042\end{array}$ & 0 & $\begin{array}{l}0.1334622 \\
0.15934\end{array}$ \\
\hline 34 & 134.94 & 50503 & 24730 & 70796 & 30218 & 896409 & 172430 & 0.040562 & 0.019862 & 0.056860 & 0.024270 & 0.719957 & 0.138488 \\
\hline & 5.00 & $\begin{array}{r}649985 \\
90503\end{array}$ & 223449 & 664406 & 237781 & 7815540 & 1529800 & 0.058447 & 0.020093 & 0.059744 & 0.021381 & 0.702776 & 37560 \\
\hline & & & $\begin{array}{l}49369 \\
50416\end{array}$ & $\begin{array}{l}139448 \\
156439\end{array}$ & & & 整3 & 63 & 0. & 0.0 & 830 & 560 & \\
\hline DSP 5 & 1355.58 & $\begin{array}{c}5544285 \\
26615\end{array}$ & 50416 & $\begin{array}{l}156449 \\
38205\end{array}$ & 70380 & 1733860 & $\begin{array}{l}3626544 \\
944138\end{array}$ & $\begin{array}{l}0.1298688 \\
0.039677\end{array}$ & $\begin{array}{l}0.0181881 \\
0.019273\end{array}$ & 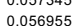 & $\begin{array}{l}0.0272437 \\
0.024\end{array}$ & $\begin{array}{l}0.032330 \\
0.7231\end{array}$ & $\begin{array}{l}0.1322336 \\
0.140339\end{array}$ \\
\hline 34 & 136.5 & 136306 & 70451 & 239493 & 102480 & 3115510 & 609966 & 0.031890 & 0.016483 & 0.056032 & 0.023976 & 0.728910 & 0.142709 \\
\hline & 136.82 & 128 & 64 & 231 & & & & & 0.0 & & 0.6 & 0.736320 & \\
\hline 34 & $\begin{array}{l}1377.13 \\
313702\end{array}$ & $\begin{array}{l}282192 \\
5575\end{array}$ & $\begin{array}{l}130518 \\
1325650\end{array}$ & 468187 & 169995 & 6139960 & 1213100 & 0.033578 & 0.015531 & 0.0557710 & 0.020228 & 0.7306044 & 0.144349 \\
\hline & & 357894 & $\begin{array}{l}132567 \\
21689\end{array}$ & $\begin{array}{l}4499735 \\
77772\end{array}$ & $\begin{array}{l}1992228 \\
{ }_{20242}\end{array}$ & 681 & $\begin{array}{l}1314280 \\
3 \\
3\end{array}$ & $\begin{array}{l}0.038845 \\
0.031512\end{array}$ & 0.0143144 & $\begin{array}{l}0.043561 \\
0.05192\end{array}$ & 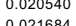 & 0.7360272 & $\begin{array}{l}0.141913 \\
0\end{array}$ \\
\hline & 9 & 22072 & $\begin{array}{l}10089 \\
10088\end{array}$ & 38588 & 02437 & 495 & $\begin{array}{l}1050649 \\
10\end{array}$ & $\begin{array}{l}0.0332151 \\
0.031\end{array}$ & $\begin{array}{l}0.01469 \\
0.04\end{array}$ & 0 & 20 & $\begin{array}{l}0.721444 \\
\end{array}$ & S4745 \\
\hline & 138.3 & 37443 & 17015 & 68900 & 300 & 9253 & 189903 & 0.029514 & 0.013412 & 0.054310 & 0.023650 & 0.729426 & 0.149689 \\
\hline & 0 & & 36422 & 106936 & 47065 & & 282860 & 0.034323 & 0.0 & 0.054892 & 0.024159 & 0.722733 & 15196 \\
\hline & & & $\begin{array}{r}31425 \\
218082\end{array}$ & $\begin{array}{l}98297 \\
81288\end{array}$ & $\begin{array}{l}46069 \\
30172\end{array}$ & & $\begin{array}{l}2487776 \\
{ }^{2}\end{array}$ & 0.0367706 & 0.0 & 94 & & 22 & \\
\hline & & & $\begin{array}{l}28082 \\
10966\end{array}$ & 81284 & $\begin{array}{l}30172 \\
{ }_{16590}\end{array}$ & & $15 / 078$ & $\begin{array}{l}0.444772 \\
0 \quad 37909\end{array}$ & 0.023729 & 70 & & $\begin{array}{l}0.45898 \\
0\end{array}$ & $\begin{array}{l}3.73730 \\
47739\end{array}$ \\
\hline & .56 & . & $\begin{array}{l}10082 \\
1008\end{array}$ & 23696 & ${ }_{13343}^{1034}$ & 0108 & $\begin{array}{l}63516 \\
6351\end{array}$ & $\begin{array}{l}0.056122 \\
0\end{array}$ & 0.021111 & $\begin{array}{l}0.049620 \\
0\end{array}$ & (944) & 0.711299 & 0.143005 \\
\hline & & & 13705 & 36746 & & 448137 & 82696 & 0.045068 & 0.021924 & 0.058782 & 256 & 16882 & 132288 \\
\hline & 31 & 550 & 605 & 545230 & & 71020 & 410812 & 0.292458 & 062918 & 063692 & 1650 & 522029 & 17909 \\
\hline & & & & & & & & & & & & & \\
\hline
\end{tabular}

\begin{tabular}{|c|c|c|c|}
\hline \multicolumn{4}{|c|}{21.39} \\
\hline & & $\begin{array}{l}16.82 \\
22.30\end{array}$ & \\
\hline 0.89 & 0.91 & 23.28 & \\
\hline 91 & $\begin{array}{l}2.01 \\
1.33\end{array}$ & $\begin{array}{l}17.18 \\
20.20\end{array}$ & 8.81 \\
\hline & & $\begin{array}{l}20.20 \\
20.20\end{array}$ & \\
\hline 98 & $\begin{array}{l}1.34 \\
1.25\end{array}$ & $\begin{array}{l}20.20 \\
21.07\end{array}$ & $\begin{array}{l}.813 \\
7.78\end{array}$ \\
\hline .9 & 1.73 & $\begin{array}{l}17.99 \\
1\end{array}$ & 8.67 \\
\hline $\begin{array}{l}0.91 \\
0.90\end{array}$ & $\begin{array}{l}1.91 \\
1.30\end{array}$ & $\begin{array}{l}17.40 \\
20.35\end{array}$ & $\begin{array}{l}8.77 \\
8.09\end{array}$ \\
\hline 0.91 & $\begin{array}{l}1.47 \\
\end{array}$ & 19.32 & 8.38 \\
\hline 0.90 & $\begin{array}{l}1.19 \\
1.55\end{array}$ & $\begin{array}{l}21.57 \\
18.58\end{array}$ & $\begin{array}{l}7.58 \\
8.59\end{array}$ \\
\hline 0.90 & 1.83 & & \\
\hline & & & \\
\hline $\begin{array}{l}0.90 \\
0.80\end{array}$ & 2.54 & 16.06 & 8.90 \\
\hline & $\begin{array}{l}2.37 \\
1.81\end{array}$ & $\begin{array}{l}10.81 \\
17.69\end{array}$ & $\begin{array}{l}8.87 \\
8.72\end{array}$ \\
\hline 8 & 1.52 & 18.97 & $\begin{array}{l}8.1 / 27 \\
8.47\end{array}$ \\
\hline $\begin{array}{l}0.91 \\
0.91\end{array}$ & 2.06 & $\begin{array}{r}17.00 \\
15.8\end{array}$ & $\begin{array}{l}8.81 \\
8.87\end{array}$ \\
\hline 0.89 & 1.83 & 17.83 & $\begin{array}{l}8.62 \\
8\end{array}$ \\
\hline $\begin{array}{l}0.88 \\
0.95\end{array}$ & $\begin{array}{l}1.525 \\
2.25\end{array}$ & $\begin{array}{l}18.44 \\
16.74\end{array}$ & $\begin{array}{l}8.1 \\
8.8\end{array}$ \\
\hline 0.96 & 2.14 & 17.21 & 8.8 \\
\hline $\begin{array}{l}0.92 \\
0.94\end{array}$ & 1.78 & $\begin{array}{l}18.52 \\
17\end{array}$ & 8.62 \\
\hline 0.89 & $\begin{array}{l}1.90 \\
1.57\end{array}$ & $\begin{array}{l}18.63 \\
18.83\end{array}$ & $\begin{array}{l}8.17 \\
8.48\end{array}$ \\
\hline 0.89 & $\begin{array}{l}1.95 \\
1.85\end{array}$ & $\begin{array}{r}17.89 \\
18.49\end{array}$ & 8.7 \\
\hline 0.93 & $\begin{array}{l}1.85 \\
1.54\end{array}$ & $\begin{array}{l}18.40 \\
19.00\end{array}$ & $\begin{array}{l}8.6 \\
8.4\end{array}$ \\
\hline $\begin{array}{l}0.91 \\
0.91\end{array}$ & 2.52 & $\begin{array}{l}15.45 \\
17\end{array}$ & 8.85 \\
\hline 0.95 & $\begin{array}{l}1.88 \\
0.88\end{array}$ & $\begin{array}{l}27.69 \\
24.46\end{array}$ & \\
\hline 0.82 & 2.10 & $\begin{array}{l}14.40 \\
16.78\end{array}$ & 8.8 \\
\hline 1.79 & 1.77 & 19.86 & 8.5 \\
\hline $\begin{array}{l}0.84 \\
0.79\end{array}$ & $\begin{array}{l}0.41 \\
2.41\end{array}$ & $\begin{array}{l}26.93 \\
16.43\end{array}$ & 888 \\
\hline 0.91 & 0.95 & 23.13 & 6.8 \\
\hline 0.92 & $\begin{array}{l}1.00 \\
1.00\end{array}$ & $\begin{array}{l}22.69 \\
23.09\end{array}$ & 6.99 \\
\hline 0.92 & 1.01 & 22.59 & 7.07 \\
\hline $\begin{array}{l}0.92 \\
0.93\end{array}$ & $\begin{array}{l}1.11 \\
1.33\end{array}$ & $\begin{array}{l}22.00 \\
20.53\end{array}$ & $\begin{array}{l}7.36 \\
7.39\end{array}$ \\
\hline 0.92 & $\begin{array}{l}1.27 \\
\end{array}$ & $\begin{array}{l}20.58 \\
21.18\end{array}$ & \\
\hline & 1.25 & 21.54 & \\
\hline 0 & $\begin{array}{l}1.135 \\
108\end{array}$ & $\begin{array}{r}20.90 \\
20,90\end{array}$ & 7.88 \\
\hline $\begin{array}{l}0.90 \\
0.92\end{array}$ & $\begin{array}{l}1.20 \\
1.25\end{array}$ & $\begin{array}{l}22.13 \\
21.3\end{array}$ & $\begin{array}{l}1.46 \\
7.6\end{array}$ \\
\hline 0.92 & 1.25 & 21.45 & 7.72 \\
\hline 0.92 & & & \\
\hline $\begin{array}{l}0.91 \\
0.92\end{array}$ & $\begin{array}{l}1.135 \\
1.35\end{array}$ & $\begin{array}{l}21.96 \\
20.6\end{array}$ & $\begin{array}{l}7.56 \\
8.8\end{array}$ \\
\hline & 1.24 & 21,4 & 80 \\
\hline 0.92 & 1.26 & 21.30 & 7.80 \\
\hline 0.92 & 1.19 & 21.66 & 7.53 \\
\hline $\begin{array}{l}0.92 \\
0.92\end{array}$ & $\begin{array}{l}1.35 \\
1.47\end{array}$ & $\begin{array}{r}20.69 \\
19.79\end{array}$ & \\
\hline 0.92 & $\begin{array}{l}1.38 \\
1.38\end{array}$ & $\begin{array}{l}20.30 \\
20.3\end{array}$ & $\begin{array}{l}8 \\
8\end{array}$ \\
\hline 0.92 & 1.22 & 21.68 & 64 \\
\hline & 1.33 & 20.76 & 7.9 \\
\hline $\begin{array}{l}0.92 \\
0.92\end{array}$ & $\begin{array}{l}1.38 \\
1.32\end{array}$ & $\begin{array}{l}20.91 \\
20.91\end{array}$ & $\begin{array}{l}8.0 \\
7.9\end{array}$ \\
\hline 0.93 & 1.49 & 19.62 & 8.2 \\
\hline $\begin{array}{l}0.94 \\
0.93\end{array}$ & 1.59 & 19.15 & \\
\hline & & 19 & 8.2 \\
\hline 0.94 & 1.52 & 10 & $\begin{array}{l}8.3 \\
8.3\end{array}$ \\
\hline 0.94 & 1.56 & 19. & 8.3 \\
\hline 0.94 & 1.59 & 18.91 & 8.4 \\
\hline $\begin{array}{l}0.92 \\
0.93\end{array}$ & $\begin{array}{l}1.47 / 2 \\
1.52\end{array}$ & 191 & 8.2 \\
\hline & $\begin{array}{l}1.15 \\
1.5\end{array}$ & & \\
\hline 0.92 & 1.65 & & \\
\hline 0.91 & 1.46 & 19.4 & 8.3 \\
\hline $\begin{array}{l}0.91 \\
0.69\end{array}$ & $\begin{array}{l}1.32 \\
0.55\end{array}$ & $\begin{array}{r}20.98 \\
2403\end{array}$ & $\begin{array}{l}7.8 \\
4.8\end{array}$ \\
\hline & 0.32 & 22.33 & 3.5 \\
\hline
\end{tabular}


Cretaceous dataset for OPTIMAL

$\begin{array}{llllllllllllll}\text { AGE (GTS 2012) } & 1302 & 1300 & 1298 & 1296 & 1292 & 1292^{\prime} & \text { Fr.1302 } & \text { Fr.1300 } & \text { Fr.1298 } & \text { Fr.1296 } & \text { Fr.1292 } & \text { Fr.1292' } & \text { TEX8 }\end{array}$

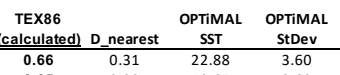

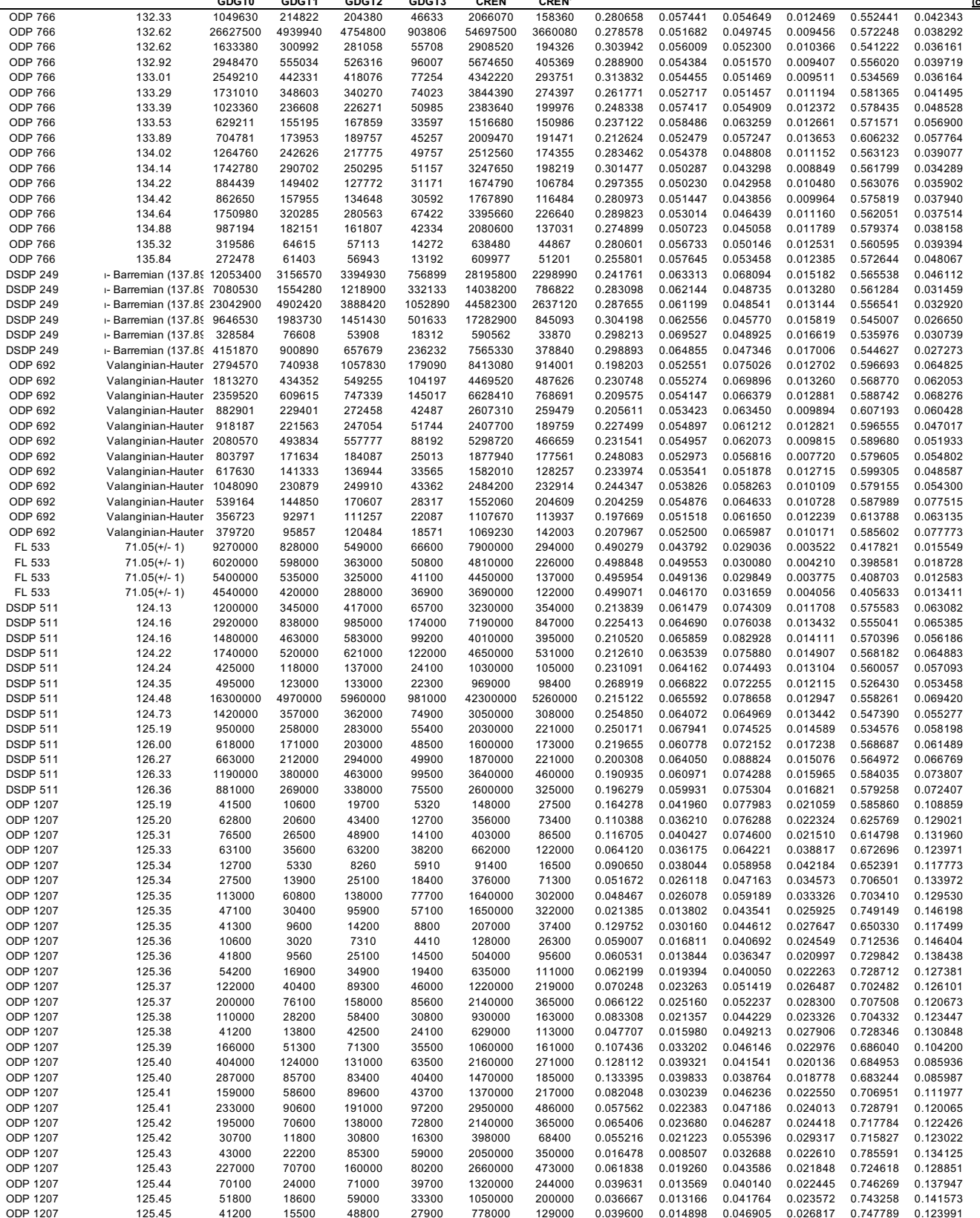

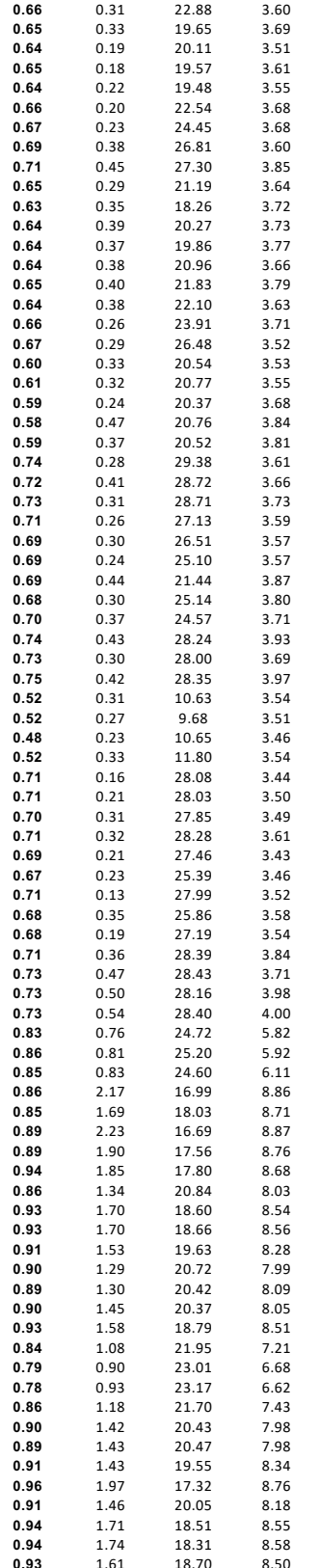


Cretaceous dataset for OPTIMAL

$\begin{array}{llllllllllllll}\text { AGE (GTS 2012) } & 1302 & 1300 & 1298 & 1296 & 1292 & 1292^{\circ} & \text { Fr.1302 } & \text { Fr.1300 } & \text { Fr. } 1298 & \text { Fr.1296 } & \text { Fr.1292 } & \text { Fr.1292 } & \text { TEX }\end{array}$

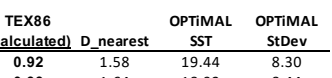

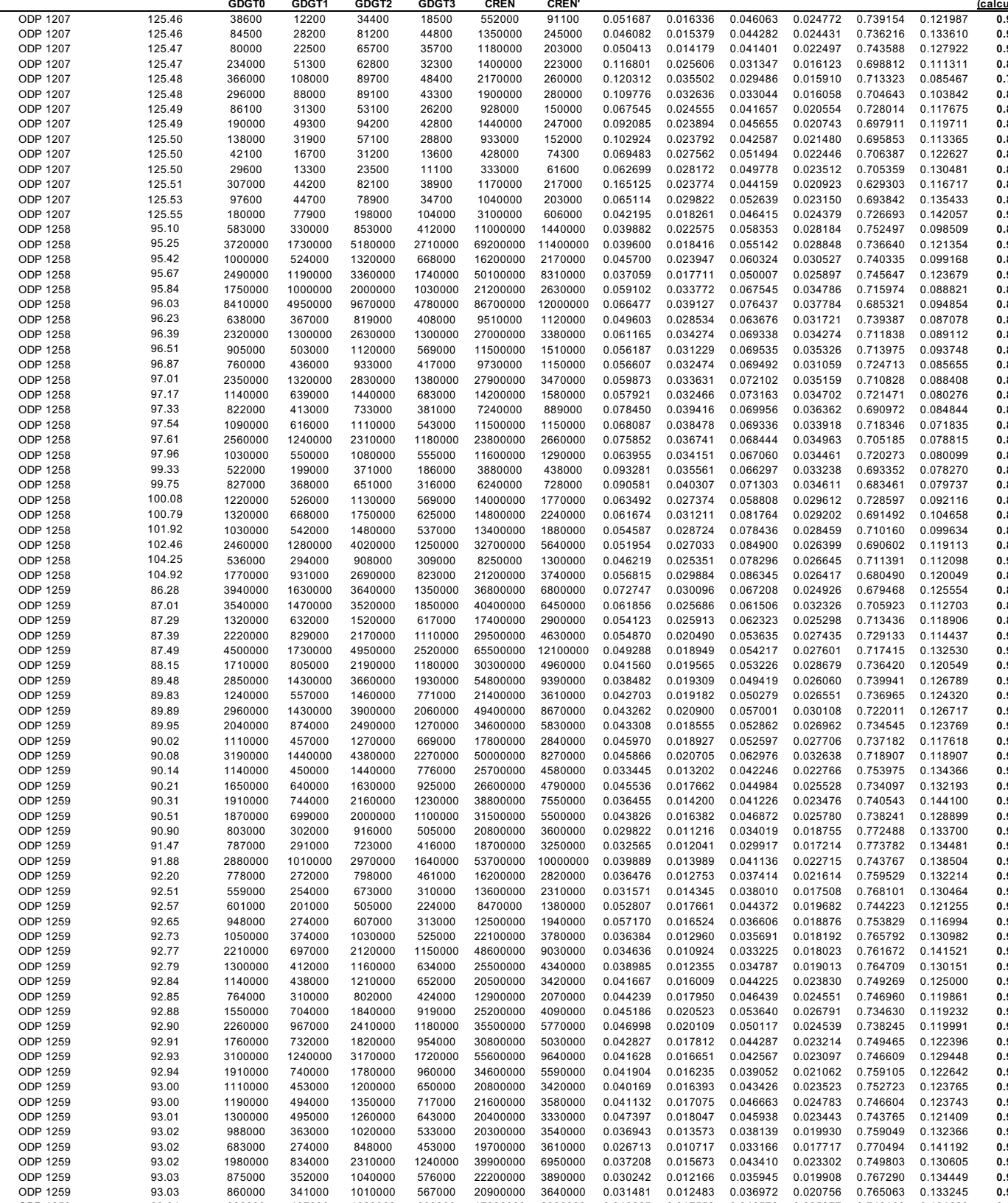

\begin{tabular}{|c|c|c|}
\hline 1.64 & & \\
\hline & & \\
\hline & 19.94 & 8.01 \\
\hline $\begin{array}{l}1.04 \\
1.21\end{array}$ & $\begin{array}{l}20.92 \\
22.100\end{array}$ & $\begin{array}{l}7.08 \\
775\end{array}$ \\
\hline 1.36 & & \\
\hline $\begin{array}{l}1.29 \\
1.33\end{array}$ & $\begin{array}{l}21.48 \\
22.101\end{array}$ & $\begin{array}{l}7.74 \\
7.83\end{array}$ \\
\hline 1.21 & $\begin{array}{l}21.91 \\
21.91\end{array}$ & $\begin{array}{l}.035 \\
7.46\end{array}$ \\
\hline $\begin{array}{l}1.30 \\
1.40\end{array}$ & $\begin{array}{l}21.14 \\
20.08\end{array}$ & $\begin{array}{l}7.74 \\
8.13\end{array}$ \\
\hline $\begin{array}{l}1.24 \\
1.54\end{array}$ & $\begin{array}{l}21.53 \\
1.93\end{array}$ & $\begin{array}{l}7.62 \\
832\end{array}$ \\
\hline $\begin{array}{l}1.59 \\
1.31\end{array}$ & 20.110 & $\begin{array}{l}8.39 \\
8.11\end{array}$ \\
\hline 1.50 & $\begin{array}{l}19.10 \\
\end{array}$ & 8.42 \\
\hline $\begin{array}{l}1.46 \\
1.51\end{array}$ & $\begin{array}{l}19.355 \\
19.39\end{array}$ & $\begin{array}{l}8.37 \\
8.31\end{array}$ \\
\hline $\begin{array}{l}1.98 \\
1.98\end{array}$ & $\begin{array}{l}18.22 \\
11822\end{array}$ & $\begin{array}{l}8.76 \\
877\end{array}$ \\
\hline $\begin{array}{l}1.50 \\
1.54\end{array}$ & $\begin{array}{l}18.22 \\
19.16\end{array}$ & $\begin{array}{l}8.47 \\
8.47\end{array}$ \\
\hline $\begin{array}{l}1.86 \\
2.08\end{array}$ & $\begin{array}{l}18.56 \\
17.71\end{array}$ & $\begin{array}{l}8.70 \\
8.80\end{array}$ \\
\hline 1.32 & $\begin{array}{l}20.49 \\
\end{array}$ & 8.11 \\
\hline $\begin{array}{l}2.02 \\
1.96\end{array}$ & $\begin{array}{l}18.21 \\
18.34\end{array}$ & $\begin{array}{l}8.77 \\
8.75\end{array}$ \\
\hline $\begin{array}{l}1.75 \\
1.83\end{array}$ & $\begin{array}{l}18.87 \\
19.92\end{array}$ & $\begin{array}{l}8.70 \\
8.62\end{array}$ \\
\hline $\begin{array}{l}1.00 \\
1.85\end{array}$ & $\begin{array}{l}18.92 \\
18.92\end{array}$ & $\begin{array}{l}8.62 \\
8.69\end{array}$ \\
\hline $\begin{array}{l}1.94 \\
1.70\end{array}$ & $\begin{array}{l}18.53 \\
19.93\end{array}$ & $\begin{array}{l}8.72 \\
88.50\end{array}$ \\
\hline $\begin{array}{l}1.71 \\
\text {. }\end{array}$ & $\begin{array}{l}19.79 \\
20.79\end{array}$ & 8.56 \\
\hline 0.86 & $\begin{array}{l}2.55 \\
23.47\end{array}$ & $\begin{array}{l}8.04 \\
6.92\end{array}$ \\
\hline & & 6.98 \\
\hline $\begin{array}{l}0.77 \\
0.89\end{array}$ & $\begin{array}{l}24.68 \\
23.51\end{array}$ & $\begin{array}{l}6.23 \\
6.78\end{array}$ \\
\hline 0.67 & 25.35 & 5.88 \\
\hline $\begin{array}{l}0.96 \\
1.64\end{array}$ & $\begin{array}{l}23.84 \\
11.72\end{array}$ & $\begin{array}{l}6.66 \\
8.58\end{array}$ \\
\hline $\begin{array}{l}1.111 \\
1.33\end{array}$ & $\begin{array}{l}22.36 \\
20.26\end{array}$ & $\begin{array}{l}7.26 \\
8.12\end{array}$ \\
\hline $\begin{array}{l}1.43 \\
.143\end{array}$ & $\begin{array}{l}19.62 \\
19.62\end{array}$ & 8.30 \\
\hline $\begin{array}{l}1.48 \\
1.50\end{array}$ & $\begin{array}{l}\begin{array}{l}19.19 \\
19.45\end{array} \\
\text { a }\end{array}$ & $\begin{array}{l}8.40 \\
8.30\end{array}$ \\
\hline $\begin{array}{r}1.46 \\
1.56\end{array}$ & $\begin{array}{l}19.55 \\
18.5\end{array}$ & 8.28 \\
\hline $\begin{array}{l}1.55 \\
1.43\end{array}$ & $\begin{array}{l}10.67 \\
19.66\end{array}$ & $\begin{array}{l}8.49 \\
8.26\end{array}$ \\
\hline $\begin{array}{l}1.42 \\
1.72\end{array}$ & $\begin{array}{l}19.58 \\
17.58\end{array}$ & $\begin{array}{l}8.29 \\
8.72\end{array}$ \\
\hline 1.70 & $\begin{array}{l}18.58 \\
18.5\end{array}$ & $\begin{array}{l}8.11 \\
8.52\end{array}$ \\
\hline $\begin{array}{l}1.60 \\
1.70\end{array}$ & $\begin{array}{l}19.05 \\
18.05\end{array}$ & $\begin{array}{l}8.42 \\
852\end{array}$ \\
\hline $\begin{array}{l}1.75 \\
1.58\end{array}$ & $\begin{array}{l}\begin{array}{l}18.31 \\
19.08\end{array}\end{array}$ & $\begin{array}{l}8.58 \\
8.41\end{array}$ \\
\hline $\begin{array}{l}1.81 \\
1.91\end{array}$ & $\begin{array}{l}18.17 \\
17.88\end{array}$ & $\begin{array}{l}8.69 \\
8.79\end{array}$ \\
\hline 1.70 & $\begin{array}{l}1,000 \\
18.58\end{array}$ & $\begin{array}{l}8.53 \\
8.53\end{array}$ \\
\hline $\begin{array}{l}1.72 \\
1.71\end{array}$ & $\begin{array}{l}18.43 \\
1888\end{array}$ & $\begin{array}{l}8.58 \\
8.68\end{array}$ \\
\hline 1.42 & 20.53 & 8.17 \\
\hline $\begin{array}{l}1.56 \\
1.73\end{array}$ & $\begin{array}{l}19.79 \\
18.63\end{array}$ & $\begin{array}{l}8.39 \\
8.63\end{array}$ \\
\hline 1.86 & 17.93 & 8.74 \\
\hline $\begin{array}{l}1.73 \\
1.61\end{array}$ & $\begin{array}{l}\text { 18.56 } \\
19.16\end{array}$ & $\begin{array}{l}8.63 \\
8.37\end{array}$ \\
\hline 1.55 & 19.55 & 8.26 \\
\hline $\begin{array}{l}1.36 \\
1.44\end{array}$ & $\begin{array}{l}\begin{array}{l}20.10 \\
20.22\end{array} \\
2\end{array}$ & $\begin{array}{l}8.13 \\
8.05\end{array}$ \\
\hline 1.54 & & \\
\hline $\begin{array}{l}1.161 \\
1.51\end{array}$ & & \\
\hline & 19.16 & 8.37 \\
\hline $\begin{array}{l}1.58 \\
1.58\end{array}$ & 19.33 & 8.32 \\
\hline & & \\
\hline 1.90 & & 8.76 \\
\hline 1.6 & $\begin{array}{l}18.96 \\
18.3\end{array}$ & \\
\hline & 18.40 & \\
\hline
\end{tabular}


Cretaceous dataset for OPTIMAL

\begin{tabular}{|c|c|c|c|c|c|c|c|c|c|c|c|c|c|c|c|c|c|}
\hline SITE & AGE (GTS 2012) & $\begin{array}{c}1302 \\
\text { GDGTO }\end{array}$ & $\begin{array}{l}1300 \\
\text { GDGT11 }\end{array}$ & $\begin{array}{c}1298 \\
\text { GDGT2 }\end{array}$ & $\begin{array}{l}1296 \\
\text { GDGT3 }\end{array}$ & $\begin{array}{l}1292 \\
\text { CREN }\end{array}$ & $\begin{array}{l}1292^{\prime} \\
\text { CREN }\end{array}$ & 302 & Fr.1300 & Fr.1298 & Fr.1296 & Fr.1292 & Fr.1292' & $\begin{array}{l}\text { TEX86 } \\
\text { (calculate }\end{array}$ & neneare & $\begin{array}{c}\text { OPPTIMAL } \\
\text { SST }\end{array}$ & $\begin{array}{l}\text { OPTiMAL } \\
\text { StDev }\end{array}$ \\
\hline $\begin{array}{l}51259 \\
5\end{array}$ & $\begin{array}{l}93.04 \\
93044\end{array}$ & $\begin{array}{l}937000 \\
1030000\end{array}$ & 364000 & 1020000 & 555000 & $\frac{18800000}{180000}$ & 31200000 & 0.037788 & 0.014680 & 0.041136 & 0.022383 & 0.758187 & 0.125827 & 0.93 & 1.62 & 18.94 & 8.45 \\
\hline & & 1040000 & 370000 & 1070000 & 595000 & 204000000 & . & 38698 & & & & & & $m_{2}>$ & 1.65 & & 8.49 \\
\hline & $\begin{array}{l}93.04 \\
9305\end{array}$ & $\begin{array}{l}978000 \\
8300000\end{array}$ & $\begin{array}{l}3450000 \\
290000\end{array}$ & $\begin{array}{l}10300000 \\
838000\end{array}$ & $\begin{array}{l}571000 \\
481000\end{array}$ & $\begin{array}{l}2110000000 \\
190000000\end{array}$ & $\begin{array}{l}35770000 \\
3270000\end{array}$ & $\begin{array}{l}0.035571 \\
0.035537\end{array}$ & $\begin{array}{l}0.012548 \\
0.0171718\end{array}$ & $\begin{array}{l}0.037463 \\
0.034476\end{array}$ & $\begin{array}{l}0.0202768 \\
0.0194355\end{array}$ & $\begin{array}{l}0.76630303 \\
0.7677708\end{array}$ & $\begin{array}{l}0.129847 \\
0.132127\end{array}$ & $\begin{array}{l}0.94 \\
0.94\end{array}$ & $\begin{array}{l}1.71 \\
1.75\end{array}$ & $\begin{array}{l}18.58 \\
18.40\end{array}$ & $\begin{array}{l}8.57 \\
8.64\end{array}$ \\
\hline & $\begin{array}{l}93.05 \\
93.05\end{array}$ & 1090000 & 386000 & 1130000 & $\begin{array}{l}4.01000 \\
633000\end{array}$ & 23900000 & $\begin{array}{l}41700000 \\
4\end{array}$ & $\begin{array}{l}.0 .035814 \\
0.03814\end{array}$ & $\begin{array}{l}.00112329 \\
0.0129\end{array}$ & $\begin{array}{l}0.00347090 \\
0.036092\end{array}$ & 0.020218 & $\begin{array}{l}0.701700 \\
0.763559\end{array}$ & 0.133189 & 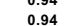 & $\begin{array}{l}1.15 \\
1.74\end{array}$ & $\begin{array}{l}18.43 \\
18.43\end{array}$ & $\begin{array}{l}8.64 \\
8.62\end{array}$ \\
\hline & 93.05 & 1440000 & 521000 & 1560000 & 844000 & 32200000 & 5670000 & $\begin{array}{l}0.034095 \\
0\end{array}$ & 0.012336 & $\begin{array}{l}0.036936 \\
0.03636\end{array}$ & 0.019983 & $\begin{array}{l}0.762401 \\
0.0501\end{array}$ & 0.134249 & 0.94 & 1.73 & 18.49 & 8.61 \\
\hline & 93.05 & 709000 & $\begin{array}{l}254000 \\
43000\end{array}$ & 762000 & 414000 & 15800000 & $\begin{array}{r}27000000 \\
287000\end{array}$ & 0.034352 & 0.012307 & 0.036920 & 0.020059 & 0.765541 & 0.130820 & 0.94 & 1.72 & 18.56 & 8.60 \\
\hline & $\begin{array}{l}93.05 \\
93.06\end{array}$ & $\begin{array}{l}11400000 \\
867000\end{array}$ & $\begin{array}{l}4330000 \\
336000\end{array}$ & $\begin{array}{l}12500000 \\
9500000\end{array}$ & $\begin{array}{l}688000 \\
524000\end{array}$ & $\begin{array}{l}229000000 \\
193000000\end{array}$ & $\begin{array}{l}38700000 \\
2510000\end{array}$ & $\begin{array}{l}0.037760 \\
0.042320\end{array}$ & $\begin{array}{l}0.014205 \\
0.0146401\end{array}$ & $\begin{array}{l}0.041294 \\
0.0463711\end{array}$ & $\begin{array}{l}0.0222497 \\
0.025577\end{array}$ & $\begin{array}{l}0.756500 \\
0.746815\end{array}$ & $\begin{array}{l}0.127745 \\
0.122517\end{array}$ & $\begin{array}{l}0.93 \\
0.92\end{array}$ & $\begin{array}{l}1.64 \\
1.58\end{array}$ & $\begin{array}{l}18.85 \\
19.13\end{array}$ & 8.47 \\
\hline & & & & & & 00 & & 35366 & & $\begin{array}{l}0.04063711 \\
0.038195\end{array}$ & $\begin{array}{l}0.02501 / 1 / \\
0.020158\end{array}$ & & $\begin{array}{l}0.122517 \\
0.129438\end{array}$ & $\begin{array}{l}0.92 \\
0.94\end{array}$ & & $\begin{array}{l}19.13 \\
18.77\end{array}$ & .38 \\
\hline $\begin{array}{l}1259 \\
1259 \\
1\end{array}$ & $\begin{array}{ll}07 \\
07\end{array}$ & 842000 & $\begin{array}{l}356000000 \\
29000\end{array}$ & $\begin{array}{l}10800000 \\
873000\end{array}$ & $\begin{array}{l}5700000 \\
476000\end{array}$ & $\begin{array}{l}216000000 \\
19200000\end{array}$ & $\begin{array}{l}352200000 \\
32200\end{array}$ & $\begin{array}{l}.0035506 \\
0.033814\end{array}$ & 011646 & $\begin{array}{l}0.0385055 \\
0.035059\end{array}$ & $\begin{array}{l}0.0219116 \\
0.0196\end{array}$ & $\begin{array}{l}0.7678599 \\
0.771053\end{array}$ & $\begin{array}{l}0.124438 \\
0.129312\end{array}$ & $\begin{array}{l}0.94 \\
0.94\end{array}$ & $\begin{array}{l}1.68 \\
1.75\end{array}$ & 18.45 & $\begin{array}{l}8.56 \\
8.64\end{array}$ \\
\hline 1259 & 93.08 & 1440000 & 560000 & 1650000 & 888000 & 338000000 & 6140000 & 0.032376 & 0.012590 & 0.037097 & 0.019965 & 0.759926 & 0.138046 & 0.94 & 1.74 & 18.39 & 8.63 \\
\hline & $\begin{array}{l}93.09 \\
93.10\end{array}$ & 80000 & $\begin{array}{l}490000 \\
31200\end{array}$ & $\begin{array}{l}14800000 \\
932000\end{array}$ & $\begin{array}{l}812000 \\
571000\end{array}$ & $\begin{array}{l}33000000 \\
18600000\end{array}$ & $\begin{array}{l}56300000 \\
31500000\end{array}$ & $\begin{array}{l}0.029982 \\
0.032682\end{array}$ & $\begin{array}{l}0.011478 \\
0.131966\end{array}$ & $\begin{array}{l}0.034667 \\
0.033314\end{array}$ & $\begin{array}{l}0.019020 \\
0.021254\end{array}$ & $\begin{array}{l}0.772979 \\
0.764645\end{array}$ & $\begin{array}{l}0.131875 \\
0.1290908\end{array}$ & 0.94 & 1.78 & $\begin{array}{l}18.28 \\
18.59\end{array}$ & 8.67 \\
\hline & & 1110000 & $\begin{array}{l}321000 \\
465000\end{array}$ & $\begin{array}{l}932000 \\
1310000\end{array}$ & & & $\begin{array}{l}\begin{array}{l}31660000 \\
3640000\end{array} \\
3\end{array}$ & 6025 & & $\begin{array}{l}0.0383814 \\
0.046057\end{array}$ & $\begin{array}{l}0.021254 \\
0.025243\end{array}$ & $\begin{array}{l}0.7646445 \\
0.745350\end{array}$ & $\begin{array}{l}0.129908 \\
0.127975\end{array}$ & .93 & $\begin{array}{l}1.69 \\
1.62\end{array}$ & $\begin{array}{l}18.59 \\
19.01\end{array}$ & $\begin{array}{l}8.56 \\
8.41\end{array}$ \\
\hline & 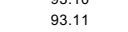 & 1260000 & $\begin{array}{l}4650000 \\
413000\end{array}$ & 1270000 & 701000 & 25400000 & 3040000 & $\begin{array}{l}.0 .039520 \\
0.037743\end{array}$ & $\begin{array}{l}.01010648 \\
0.012371\end{array}$ & $\begin{array}{l}0.040005 / \\
0.038042\end{array}$ & $\begin{array}{l}0.02020243 \\
0.020998\end{array}$ & $\begin{array}{l}0.14508500 \\
0.760844\end{array}$ & $\begin{array}{l}0.130002 \\
0\end{array}$ & $\begin{array}{l}0.94 \\
0.94\end{array}$ & $\begin{array}{l}1.1 .70 \\
1.70\end{array}$ & $\begin{array}{l}19.01 \\
18.61\end{array}$ & $\begin{array}{l}8.41 \\
8.56\end{array}$ \\
\hline & & 1100000 & 397000 & 1110000 & 604000 & 17800000 & 2910000 & 0.045985 & 0.016596 & 0.046403 & 0.025250 & 0.744116 & 0.121650 & 0.92 & 1.57 & 19.30 & 8.34 \\
\hline & $\begin{array}{l}111.03 \\
111.04\end{array}$ & 61675980 & 21854366 & 28357604 & 10627790 & 182420326 & 26029842 & 0.186351 & 0.066032 & 0.085681 & 0.032111 & 0.551176 & 0.078648 & 0.75 & 1.38 & 22.01 & 7.88 \\
\hline & $\begin{array}{l}\begin{array}{l}111.04 \\
111.05\end{array} \\
\end{array}$ & $\begin{array}{l}76928227 \\
571622760\end{array}$ & $\begin{array}{l}28286833 \\
211006926\end{array}$ & $\begin{array}{l}37200523 \\
26878666\end{array}$ & $\begin{array}{l}14556611 \\
10712880\end{array}$ & $\begin{array}{l}238536464 \\
174961638\end{array}$ & $\begin{array}{l}\begin{array}{l}32598775 \\
25559510\end{array} \\
2\end{array}$ & $\begin{array}{l}0.179694 \\
0.1850559\end{array}$ & $\begin{array}{l}0.066074 \\
0.063654\end{array}$ & $\begin{array}{l}0.086895 \\
00894901\end{array}$ & $\begin{array}{l}0.034002 \\
0.038356\end{array}$ & $\begin{array}{l}0.557188 \\
0.555648\end{array}$ & $\begin{array}{l}0.076146 \\
0.081682\end{array}$ & 0.75 & 1.04 & $\begin{array}{l}22.18 \\
21.91\end{array}$ & 7.52 \\
\hline & .05 & $\begin{array}{l}577626760 \\
58006773\end{array}$ & $\begin{array}{l}\begin{array}{l}210066926 \\
20024099\end{array} \\
20\end{array}$ & $\begin{array}{l}26878666 \\
26146872\end{array}$ & & $\begin{array}{l}1749618322 \\
177813114\end{array}$ & $\begin{array}{l}255559510 \\
26454920\end{array}$ & $\begin{array}{l}0.0180559 \\
0.182225\end{array}$ & $\begin{array}{l}.0 .066354 \\
0.062905\end{array}$ & $\begin{array}{l}.0 .084901 \\
0.082139\end{array}$ & $\begin{array}{l}0.038356 \\
0.031035\end{array}$ & $\begin{array}{l}0.5552648 \\
0.558590\end{array}$ & $\begin{array}{l}0.081682 \\
0.083107\end{array}$ & $\begin{array}{l}0.75 \\
0.76\end{array}$ & $\begin{array}{l}1.11 \\
1.29\end{array}$ & $\begin{array}{l}\begin{array}{l}21.91 \\
22.55\end{array} \\
2.5\end{array}$ & .69 \\
\hline & & 154999475 & & & & & & & & & & 0.597759 & 0.085646 & 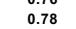 & 1.25 & 21.88 & 96 \\
\hline 545 & .07 & 124100295 & 50485147 & 73088728 & 29834635 & 524663997 & 68720050 & 0.142498 & 0.057969 & 0.083924 & 0.034258 & 0.602444 & 0.078908 & 0.77 & 1.17 & 22.20 & 7.78 \\
\hline 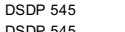 & $\begin{array}{l}111.08 \\
11110\end{array}$ & 27679 & $\begin{array}{l}360689937 \\
40202645\end{array}$ & $\begin{array}{l}537539344 \\
64556190\end{array}$ & $\begin{array}{ll}6823 \\
68039\end{array}$ & 8844 & 22 & $\begin{array}{l}0.139337 \\
\end{array}$ & $\begin{array}{l}0.057093 \\
0.055550\end{array}$ & $\begin{array}{l}0.0855086 \\
\end{array}$ & $\begin{array}{l}0.033520 \\
0.025670\end{array}$ & 0.602790 & 0.082173 & 0.78 & 1.32 & 22.21 & .92 \\
\hline & 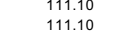 & $\begin{array}{l}1167 \\
156987\end{array}$ & & & & & & 125891 & $\begin{array}{l}0.05555050 \\
0.05011\end{array}$ & $\begin{array}{l}0.0877189 \\
0.081488\end{array}$ & $\begin{array}{l}0.035677 \\
0.036068\end{array}$ & $\begin{array}{l}0.606162 \\
0.617578\end{array}$ & $\begin{array}{l}0.084949 \\
0.083965\end{array}$ & $\begin{array}{l}0.7 \\
0.7\end{array}$ & $\begin{array}{l}1.18 \\
1.16\end{array}$ & $\begin{array}{l}57 \\
53\end{array}$ & $\begin{array}{l}97 \\
95\end{array}$ \\
\hline & & 70329397 & $\begin{array}{l}31004666 \\
3106\end{array}$ & & & & & 128525 & & & & & & & $\begin{array}{l}1.16 \\
1.21\end{array}$ & & 95 \\
\hline $\begin{array}{l}\text { P 545 } \\
\text { P }\end{array}$ & 111.12 & 72705440 & 29932141 & 46015666 & 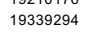 & $\begin{array}{l}343248412 \\
3\end{array}$ & $\begin{array}{l}40167607 \\
4916727\end{array}$ & $\begin{array}{l}.012529 \\
0.129736\end{array}$ & $\begin{array}{l}0.00000000 \\
0.053411\end{array}$ & 0.082111 & $\begin{array}{l}0.037300 \\
0.034509\end{array}$ & $\begin{array}{l}0.014248 \\
0.612497\end{array}$ & 0.087735 & 0.18 & $\begin{array}{l}1.21 \\
1.33\end{array}$ & $\begin{array}{l}21.54 \\
21.54\end{array}$ & .11 \\
\hline 545 & 111.13 & 67724634 & $\begin{array}{r}28954680 \\
\end{array}$ & 43793501 & 18673669 & 1659 & 8707 & 0.127677 & 0.054586 & 0.082561 & 0.035204 & 0.612555 & 0.087416 & 0.79 & 1.26 & 21.41 & .08 \\
\hline & & $\begin{array}{l}43905925 \\
41552289\end{array}$ & $\begin{array}{l}17327691 \\
14701058\end{array}$ & $\begin{array}{l}25299868 \\
16574112\end{array}$ & $\begin{array}{l}10696010 \\
6394358\end{array}$ & & & $\begin{array}{l}0.140826 \\
0.917994\end{array}$ & $\begin{array}{l}0.055578 \\
0.6775656\end{array}$ & $\begin{array}{l}0.081148 \\
0.075502\end{array}$ & $\begin{array}{l}0.034307 \\
0.025915\end{array}$ & $\begin{array}{l}0.599466 \\
0.5657272\end{array}$ & 0.088676 & & 1.28 & 74 & 02 \\
\hline & 11 & $\begin{array}{l}415522899 \\
35251314\end{array}$ & $\begin{array}{l}\text { 14701058 } \\
121111329\end{array}$ & $\begin{array}{l}16574112 \\
147074104\end{array}$ & $\begin{array}{l}63934358 \\
{ }_{535147991}\end{array}$ & $\begin{array}{l}121915258 \\
101793415\end{array}$ & $\begin{array}{l}{ }_{151513651} \\
{ }_{32325344}\end{array}$ & $\begin{array}{l}0.191794 \\
0.193903\end{array}$ & $\begin{array}{l}0.0678566 \\
0.066620\end{array}$ & $\begin{array}{l}0.076502 \\
0.077416\end{array}$ & $\begin{array}{l}0.029915 \\
0.0229235\end{array}$ & $\begin{array}{l}0.5652727 \\
0.559925\end{array}$ & $\begin{array}{l}0.071607 \\
0.072901\end{array}$ & $\begin{array}{l}0.72 \\
0.73\end{array}$ & $\begin{array}{l}1.13 \\
1.08\end{array}$ & $\begin{array}{l}22.36 \\
22.61\end{array}$ & $\begin{array}{l}.46 \\
34\end{array}$ \\
\hline$P$ P 545 & 111.15 & 36853279 & 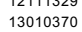 & 9508 & $\begin{array}{l}\begin{array}{l}351449191 \\
6127170\end{array} \\
6\end{array}$ & 108804091 & 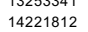 & $\begin{array}{l}.0193903 \\
0.189383\end{array}$ & $\begin{array}{l}.000006858 \\
0.06858\end{array}$ & 0.080061 & $\begin{array}{l}0.031487 \\
0.03145\end{array}$ & 0 & 0.073084 & 0.73 & $\begin{array}{l}1.00 \\
1.43\end{array}$ & $\begin{array}{l}22.01 \\
22.00\end{array}$ & $\begin{array}{l}7.34 \\
7.84\end{array}$ \\
\hline & 111.15 & 51461349 & 18290060 & 22299327 & 8653004 & 154064992 & 19678814 & 0.187509 & 0.066643 & 0.081252 & 0.031529 & 0.561364 & 0.071703 & 0.1. & 1.42 & 22.10 & .80 \\
\hline & & 327 & 289 & & $\begin{array}{l}4710970 \\
\end{array}$ & $\begin{array}{l}89265949 \\
71100777\end{array}$ & & 963 & 272 & 68 & 557 & 274 & 3366 & & 1.13 & $=$ & 44 \\
\hline & $\begin{array}{c}11 \\
69 \\
69\end{array}$ & $\begin{array}{c}255726470 \\
788000\end{array}$ & $\begin{array}{l}8614830 \\
326000\end{array}$ & $\begin{array}{l}97077315 \\
363000\end{array}$ & $\begin{array}{l}3688674 \\
1122000\end{array}$ & $\begin{array}{l}71607679 \\
19300000\end{array}$ & $\begin{array}{l}90212229 \\
279000\end{array}$ & $\begin{array}{l}0415 \\
66933 \\
\end{array}$ & $\begin{array}{l}0.0677111 \\
0.085609\end{array}$ & $\begin{array}{l}0.075622 \\
0.095326\end{array}$ & $\begin{array}{l}0.028736 \\
0.032038\end{array}$ & $\begin{array}{l}0.5577399 \\
0.5068828\end{array}$ & $\begin{array}{l}0.070277 \\
0.072367\end{array}$ & 0.72 & $\begin{array}{l}1.07 \\
1.56\end{array}$ & & .30 \\
\hline & & $\begin{array}{l}888000 \\
816000\end{array}$ & $\begin{array}{l}3260000 \\
294000\end{array}$ & & $\begin{array}{l}122000 \\
118000\end{array}$ & $\begin{array}{l}19300000 \\
1830000\end{array}$ & $\begin{array}{l}279000 \\
327000\end{array}$ & $\begin{array}{l}60243 \\
0.243\end{array}$ & $\begin{array}{l}.00856099 \\
0.079352\end{array}$ & $\begin{array}{l}.0 .0935326 \\
0.086370\end{array}$ & $\begin{array}{l}0.03032038 \\
0.031849\end{array}$ & 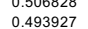 & $\begin{array}{l}0.073267 \\
0.088259\end{array}$ & & $\begin{array}{l}1.56 \\
1.62\end{array}$ & 65 & $\begin{array}{l}46 \\
47\end{array}$ \\
\hline $\mathrm{B}$ & 69 & $286 \mathrm{C}$ & & & & 00 & & 6780 & 0.075685 & 0.081189 & 0.031237 & 0.545617 & 0.069492 & & & 21 & .08 \\
\hline & 69 & 205000 & & & & & & & 548 & 0.079155 & 0.025379 & 0.570592 & 2411 & & 64 & 54 & 59 \\
\hline & $\begin{array}{ll}69.26 \\
69.3\end{array}$ & $\begin{array}{l}696000 \\
505000\end{array}$ & $\begin{array}{l}267000 \\
194000\end{array}$ & $\begin{array}{l}314000 \\
255000\end{array}$ & $\begin{array}{l}97700 \\
89500\end{array}$ & $\begin{array}{l}1540000 \\
13100000\end{array}$ & $\begin{array}{l}223000 \\
243000\end{array}$ & 0.221819 & $\begin{array}{l}0.085094 \\
\end{array}$ & 0.100073 & 0.031137 & $\begin{array}{l}0.490805 \\
0\end{array}$ & 0.071071 & 0.70 & 1.75 & 19.24 & 55 \\
\hline & & & & & & 714000 & & 2795 & $\begin{array}{l}953 \\
156\end{array}$ & 0.077046 & 0.028702 & $\begin{array}{l}\begin{array}{l}0.506124 \\
0.572436\end{array} \\
0.56\end{array}$ & 8884 & & & & 17 \\
\hline 年 & 69.43 & 852000 & $\begin{array}{l}90000 \\
289000\end{array}$ & 300000 & $\begin{array}{l}505000 \\
105000\end{array}$ & $\begin{array}{l}7144000 \\
1490000\end{array}$ & $\begin{array}{l}83400 \\
195000\end{array}$ & 3695 & 0.089446 & $\begin{array}{l}.071040 \\
0.092851\end{array}$ & 2498 & $\begin{array}{l}0.0727450 \\
0.461158\end{array}$ & $\begin{array}{l}0.0606064 \\
0.060353\end{array}$ & & 1.02 & $\begin{array}{l}22.41 \\
18.09\end{array}$ & $\begin{array}{l}17 \\
68\end{array}$ \\
\hline$B$ & 0.00 & 1240000 & 000 & 472000 & 157000 & 2320000 & 306000 & 49547 & 0.095391 & 0.094989 & 0.031596 & 0.466895 & 0.061582 & 0.66 & 1.87 & 17.78 & .76 \\
\hline 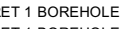 & 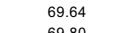 & $\begin{array}{l}986000 \\
751000\end{array}$ & 25000 & $\begin{array}{l}343000 \\
25000\end{array}$ & 09000 & 0 & & 0.269252 & 0.092845 & 0.0900000 & 0.034134 & 0.450573 & 0.059530 & 0.0 & 1.59 & - & 62 \\
\hline & & & & & & & & 997 & & & & & & & & & 71 \\
\hline BOREAC & $\begin{array}{l}69 \\
70\end{array}$ & $\begin{array}{l}8550000 \\
477000\end{array}$ & $\begin{array}{l}2790000 \\
171000\end{array}$ & $\begin{array}{l}\begin{array}{l}253000 \\
174000\end{array} \\
0\end{array}$ & $\begin{array}{l}85500 \\
62200\end{array}$ & $\begin{array}{l}11440000 \\
865000\end{array}$ & $\begin{array}{l}149000 \\
135000\end{array}$ & $\begin{array}{l}0.308340 \\
0.253158\end{array}$ & $\begin{array}{l}0.1011208 \\
0.090755\end{array}$ & $\begin{array}{l}.0 .991776 \\
0.092347\end{array}$ & $\begin{array}{l}0.0303088 \\
0.033011\end{array}$ & $\begin{array}{l}5088 \\
081\end{array}$ & $\begin{array}{l}050 \\
648\end{array}$ & $\begin{array}{l}0.64 \\
0.68\end{array}$ & 33 & 然 & 88 \\
\hline $\mathrm{E}$ & 70 & 981000 & 00 & 401000 & 145000 & 1960000 & 313000 & $\begin{array}{l}158 \\
633\end{array}$ & 127 & 910 & 681 & $\begin{array}{l}0.456087 \\
0.468787\end{array}$ & 1862 & $\begin{array}{l}0.68 \\
0.69\end{array}$ & $\begin{array}{l}1.64 \\
1.42\end{array}$ & $\begin{array}{l}18.17 \\
18.78\end{array}$ & $\begin{array}{l}65 \\
43\end{array}$ \\
\hline$B C$ & 70.48 & 1310000 & 445000 & 454000 & 172000 & 2300000 & 301000 & 0.262947 & 0.089322 & 0.091128 & 0.034524 & 0.461662 & 0.060418 & 0.6 & 1.44 & 01 & 45 \\
\hline & 70 & & & & & & & & & & & & & & & & \\
\hline BC & 70. & & $\begin{array}{l}133000 \\
132000\end{array}$ & $\begin{array}{l}1557000 \\
143000\end{array}$ & & $\begin{array}{l}957000 \\
872000\end{array}$ & & $\begin{array}{ll}840 \\
302\end{array}$ & $\begin{array}{l}0.0720799 \\
0.079582\end{array}$ & & 0. & 426 & & & & & .01 \\
\hline & 10 & & 132000 & 1430 & & 1890000 & 101000 & & & & & & & & & & 8.21 \\
\hline & $70.7 \mathrm{Y}=0$ & 393000 & & 14 & 5350 & 910000 & 109 & 37 & 0.07 & & & & & & & & 29 \\
\hline & 70. & & & & & & & & & & & & & & & & 28 \\
\hline & & & & & & & & & & & & & & & & & 16 \\
\hline But & & & & & & 60 & & & & & & & & & & & 78 \\
\hline & 71 & & & & & & & & & & & & & & & & $\begin{array}{l}8.01 \\
7.20\end{array}$ \\
\hline BO & 71 & 287000 & 100000 & 12 & & 7 & 12. & 726 & 0.068894 & 0.0 & 036 & 241 & 29 & & & & \\
\hline & 71 & & & 70 & & & 80 & & $\begin{array}{l}0.079472 \\
\end{array}$ & 0.086220 & 915 & 6 & & & & & 资3 \\
\hline & $\begin{array}{l}11.24 \\
71.28\end{array}$ & & & & & & & & & & & & & & & & \\
\hline & 11. & $\begin{array}{l}609000 \\
60000\end{array}$ & & 233000 & & 273000 & & & & & & & & & & & $\begin{array}{l}7.40 \\
8.88\end{array}$ \\
\hline & 71 & 000 & & & & & & & & & & & & & & & .07 \\
\hline & 71. & 253000 & 92200 & 100000 & & 601 & 72400 & 19371 & & 0. & 88 & 3 & 0 & & & 20.28 & 28 \\
\hline & & & & & & & & & & & & & & & & & \\
\hline & & & & & & & & & & & & & & & & & $\begin{array}{l}.03 \\
.64\end{array}$ \\
\hline & & & & & & & & & & & & & & & & & 64 \\
\hline & & 839000 & & & & 16 & & & & & & & & & & & \\
\hline & & & & & & & & & & & & & & & & & 19 \\
\hline & 3.79 & & & & & & & & & & & & & & & & \\
\hline & & & & & & & & & & & & & & & & & \\
\hline & & & & & & & & & 681 & 173768 > & 31139 & 77 & & & & & \\
\hline & & 000 & 400 & 00 & 6200 & 1000 & & & & & & & & 1.72 & 0.90 & 22:.90 & os \\
\hline
\end{tabular}


Cretaceous dataset for OPTIMAL

\begin{tabular}{|c|c|c|c|c|c|c|c|c|c|c|c|c|c|c|c|c|c|}
\hline SITE & AGE (GTS 2012) & $\begin{array}{l}1302 \\
\text { GDGTO }\end{array}$ & $\begin{array}{l}1300 \\
\text { GDGT1 }\end{array}$ & $\begin{array}{c}1298 \\
\text { GDGT2 }\end{array}$ & $\begin{array}{c}1296 \\
\text { GDGT3 }\end{array}$ & $\begin{array}{l}1292 \\
\text { CREN }\end{array}$ & $\begin{array}{l}1292 \cdot \\
\text { CREN }\end{array}$ & Fr.1302 & Fr.1300 & Fr. 1298 & Fr. 1296 & Fr. 1292 & Fr.1292: & $\begin{array}{c}\text { TEX86 } \\
\text { (calculate }\end{array}$ & & $\begin{array}{l}\text { OPTIMAL } \\
\text { SST }\end{array}$ & $\begin{array}{l}\text { OPTiMAL } \\
\text { StDev }\end{array}$ \\
\hline BO & 75.83 & & 104000 & 106000 & 40100 & $\begin{array}{l}\frac{C R E N}{764000} \\
\end{array}$ & $\begin{array}{l}\text { CREN } \\
107000\end{array}$ & 0.199843 & 0.074227 & 0.075655 & 0.028620 & 0.545286 & 0.076369 & aldua & neare $\frac{\text { n.17 }}{1.17}$ & $\begin{array}{l}351 \\
21.80\end{array}$ & \\
\hline & 75.89 & 223000 & 72800 & 79000 & 35200 & 685000 & 72800 & 0.190957 & 0.0623399 & 0.067649 & 42 & 173 & 0.062339 & 0.72 & 1.23 & 22.01 & 7.64 \\
\hline BOREHOLE & 75.95 & 190000 & 68600 & 80600 & $\begin{array}{l}32200 \\
3990\end{array}$ & $\begin{array}{l}652000 \\
112000\end{array}$ & $\begin{array}{l}65300 \\
13000\end{array}$ & 0.174520 & 0.063011 & 0.074033 & 0.029577 & 0.5988779 & 0.059980 & 0.72 & 1.02 & 22.83 & 7.12 \\
\hline $\begin{array}{l}\text { OREHOLE } \\
\text { OOEHHOE }\end{array}$ & $\begin{array}{l}76.18 \\
7648\end{array}$ & $\begin{array}{l}39100 \\
76600\end{array}$ & $\begin{array}{l}16700 \\
28300\end{array}$ & $\begin{array}{l}18000 \\
22500\end{array}$ & $\begin{array}{l}8940 \\
10700\end{array}$ & $\begin{array}{l}142000 \\
157700\end{array}$ & $\begin{array}{l}13900 \\
14100\end{array}$ & $\begin{array}{l}0.163845 \\
0.24736\end{array}$ & $\begin{array}{l}0.069980 \\
0.09157\end{array}$ & $\begin{array}{l}0.075427 \\
0.072768\end{array}$ & 0.037462 & 0.595039 & $\begin{array}{l}0.058247 \\
0.045602\end{array}$ & $\begin{array}{l}0.71 \\
0.63\end{array}$ & 0.48 & $\begin{array}{l}24.20 \\
19.13\end{array}$ & 5.71 \\
\hline 1 BOREHOLE & .76 & 77700 & $\begin{array}{l}278500 \\
27700\end{array}$ & $\begin{array}{l}225000 \\
2000\end{array}$ & $\begin{array}{l}107000 \\
8130\end{array}$ & $\begin{array}{l}15 / 5000 \\
131000\end{array}$ & $\begin{array}{l}14100 \\
12100\end{array}$ & $\begin{array}{l}0.280881 \\
0.28081\end{array}$ & $\begin{array}{l}0.091527 \\
0.100134\end{array}$ & 0.072299 & 0.029389 & $\begin{array}{l}0.5073557 \\
0.4357\end{array}$ & $\begin{array}{l}0.043741 \\
0.04602\end{array}$ & $\begin{array}{l}0.63 \\
0.59\end{array}$ & $\begin{array}{l}1.30 \\
2.20\end{array}$ & $\begin{array}{l}19.13 \\
17.02\end{array}$ & $\begin{array}{l}8.25 \\
8.82\end{array}$ \\
\hline BOREHOLE & 77.15 & 27700 & 10800 & 9350 & 7530 & 119000 & 13700 & 0.147278 & 0.057422 & 0.049713 & 0.040036 & 0.632709 & 0.072841 & 0.74 & 1.13 & 22.82 & 7.47 \\
\hline & $\begin{array}{l}77.45 \\
7774\end{array}$ & $\begin{array}{l}650000 \\
77300\end{array}$ & $\begin{array}{l}234000 \\
23990\end{array}$ & $\begin{array}{l}274000 \\
20600\end{array}$ & $\begin{array}{l}89400 \\
14400\end{array}$ & $\begin{array}{l}1450000 \\
258500\end{array}$ & $\begin{array}{l}217000 \\
24400\end{array}$ & $\begin{array}{l}0.223030 \\
0.180166\end{array}$ & 0.080291 & 0.094016 & $\begin{array}{l}0.030675 \\
0.03334\end{array}$ & $\begin{array}{l}0.497530 \\
0.593649\end{array}$ & 0.074458 & 0.71 & 1.67 & 19.89 & 8.43 \\
\hline & $\begin{array}{l}77.74 \\
78.03\end{array}$ & $\begin{array}{l}78300 \\
473000\end{array}$ & $\begin{array}{l}29900 \\
187000\end{array}$ & & $\begin{array}{l}14400 \\
74500\end{array}$ & $\begin{array}{l}258000 \\
1140000\end{array}$ & $\begin{array}{l}24400 \\
184000\end{array}$ & & $\begin{array}{l}0.068799 \\
0.082470\end{array}$ & $\begin{array}{l}0.068109 \\
0.092172\end{array}$ & $\begin{array}{l}0.033134 \\
0.032856\end{array}$ & $\begin{array}{l}0.593649 \\
0.502756\end{array}$ & $\begin{array}{l}0.056144 \\
0.081147\end{array}$ & $\begin{array}{l}0.70 \\
0.71\end{array}$ & $\begin{array}{l}1.04 \\
1.43\end{array}$ & $\begin{array}{l}22.00 \\
1977\end{array}$ & 35 \\
\hline & $\begin{array}{l}78.03 \\
78.91\end{array}$ & $\begin{array}{l}\begin{array}{l}4733000 \\
540000\end{array} \\
\end{array}$ & $\begin{array}{l}\begin{array}{l}8877000 \\
145000\end{array} \\
\end{array}$ & $\begin{array}{l}209000 \\
175000\end{array}$ & $\begin{array}{l}7 \begin{array}{l}77500 \\
61800\end{array} \\
\end{array}$ & $\begin{array}{l}\begin{array}{l}11440000 \\
1100000\end{array} \\
\end{array}$ & $\begin{array}{l}1840000 \\
168000\end{array}$ & $\begin{array}{l}0.208600 \\
0.246598\end{array}$ & $\begin{array}{l}0.082470 \\
0.066216\end{array}$ & $\begin{array}{l}0.0979916 \\
0.07916\end{array}$ & $\begin{array}{l}0.0382822 \\
0.02822\end{array}$ & 0.502329 & $\begin{array}{l}0.076719 \\
0.08714\end{array}$ & $\begin{array}{l}0.74 \\
0.74\end{array}$ & $\begin{array}{l}1.43 \\
1.41\end{array}$ & $\begin{array}{l}19.77 \\
21.13\end{array}$ & 035 \\
\hline LE & .50 & 287000 & 100000 & 121000 & 46600 & 774000 & 124000 & 77 & 0.068842 & 0.083299 & 0.032080 & 0.532838 & 0.085364 & 0.74 & 1.44 & 21.32 & 09 \\
\hline EHOLE & 80.21 & 109000 & 37000 & 41400 & 15700 & 318000 & 37700 & 0.195061 & 0.066213 & 0.074087 & 0.028096 & 0.569077 & 0.067466 & 0.72 & 0.95 & 23.00 & 89 \\
\hline LEF & 81.93 & $\begin{array}{l}213000 \\
105000\end{array}$ & $\begin{array}{l}75700 \\
32800\end{array}$ & $\begin{array}{l}88200 \\
48900\end{array}$ & $\begin{array}{l}36200 \\
18500\end{array}$ & & $\begin{array}{l}82400 \\
56000\end{array}$ & $\begin{array}{l}0.189924 \\
0.074492\end{array}$ & & $\begin{array}{l}0.078645 \\
0.081473\end{array}$ & $\begin{array}{l}0.032278 \\
0.030823\end{array}$ & $\begin{array}{l}0.558181 \\
0.568412\end{array}$ & 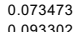 & 0.73 & 1.30 & .83 & \\
\hline OOREHOLE & $\begin{array}{l}81.93 \\
83.11\end{array}$ & $\begin{array}{l}105000 \\
106000\end{array}$ & $\begin{array}{l}32800 \\
36100\end{array}$ & $\begin{array}{l}48900 \\
51200\end{array}$ & $\begin{array}{l}18500 \\
25000\end{array}$ & $\begin{array}{l}3390000 \\
346000\end{array}$ & $\begin{array}{l}56000 \\
73600\end{array}$ & $\begin{array}{l}\begin{array}{l}0.1744942 \\
0.166170\end{array} \\
\text { a }\end{array}$ & $\begin{array}{l}0.054648 \\
0.056592\end{array}$ & $\begin{array}{l}0.081473 \\
0.080263\end{array}$ & $\begin{array}{l}0.030823 \\
0.039191\end{array}$ & $\begin{array}{l}0.564812 \\
0.542405\end{array}$ & $\begin{array}{l}0.093302 \\
0.115379\end{array}$ & $\begin{array}{l}0.79 \\
0.81\end{array}$ & $\begin{array}{l}1.22 \\
1.39\end{array}$ & $\begin{array}{l}23.18 \\
20.05\end{array}$ & 37 \\
\hline ILLE & 83.62 & 125000 & 30900 & $\begin{array}{l}51200 \\
46500\end{array}$ & 16700 & 302000 & 47300 & 0.219916 & 0.054363 & 0.081809 & 0.029381 & 0.531316 & 0.0832116 & 0 & 1.24 & 22.32 & 72 \\
\hline REHOLE & 03 & 79000 & 28900 & 46700 & 15600 & 3600000 & 51500 & 0.135809 & $\begin{array}{l}0.049682 \\
0.0490\end{array}$ & 0.080282 & 0.026818 & 0.618876 & $\begin{array}{l}0.088534 \\
0\end{array}$ & 0.80 & 0.38 & 26.39 & 63 \\
\hline & 84.55 & $\begin{array}{l}65100 \\
5\end{array}$ & $\begin{array}{r}26400 \\
23100\end{array}$ & $\begin{array}{r}39200 \\
37330\end{array}$ & $\begin{array}{l}13400 \\
14100\end{array}$ & $\begin{array}{l}340000 \\
322000\end{array}$ & $\begin{array}{l}63700 \\
65100\end{array}$ & 0.118839 & 0.048193 & $\begin{array}{l}0.071559 \\
\end{array}$ & 0.024461 & 0.620664 & 0.116283 & 0.81 & 0.69 & 25.01 & 5.69 \\
\hline & $\begin{array}{l}84.95 \\
70.36\end{array}$ & $\begin{array}{r}51300 \\
1030000\end{array}$ & $\begin{array}{r}23100 \\
365000\end{array}$ & $\begin{array}{r}373000 \\
446000\end{array}$ & $\begin{array}{l}141000 \\
135000\end{array}$ & $\begin{array}{l}3220000 \\
1650000\end{array}$ & $\begin{array}{r}65100 \\
289000\end{array}$ & $\begin{array}{l}0.100019 \\
0.263091\end{array}$ & $\begin{array}{l}0.045038 \\
0.099232\end{array}$ & $\begin{array}{l}0.072724 \\
0.1119292\end{array}$ & $\begin{array}{l}0.027491 \\
0.03448\end{array}$ & $\begin{array}{l}0.627803 \\
0.421456\end{array}$ & $\begin{array}{l}0.126925 \\
0.0783819\end{array}$ & $\begin{array}{l}0.83 \\
0.70\end{array}$ & $\begin{array}{l}0.76 \\
1.75\end{array}$ & 4.83 & 41 \\
\hline & & & & & 135000 & & 289000 & & $\begin{array}{l}0.0992351 \\
0.090963\end{array}$ & $\begin{array}{l}0.117927 \\
0.081033\end{array}$ & $\begin{array}{l}4.483 \\
9196\end{array}$ & $\begin{array}{l}0.421456 \\
0.415094\end{array}$ & $\begin{array}{l}0.078519 \\
0.054022\end{array}$ & 0.10 & & & 82 \\
\hline UARRY & 70.46 & 1660000 & 593000 & 757000 & 222000 & 3040000 & 517000 & 0.244513 & 0.0877347 & 0.111504 & 0.032700 & 0.447783 & 0.076153 & 0.72 & $\begin{array}{l}2.774 \\
1.74\end{array}$ & 18.12 & 70 \\
\hline RY & 70.49 & 33700 & 11200 & 10800 & 3450 & 54100 & 8650 & 0.276456 & 0.091879 & 0.088597 & 0.028302 & 0.443806 & 0.070960 & 0.67 & 1.88 & 18.25 & 71 \\
\hline & $\begin{array}{l}70 . \\
70\end{array}$ & $\begin{array}{l}1490000 \\
408000\end{array}$ & $\begin{array}{l}510000 \\
121000\end{array}$ & $\begin{array}{l}600000 \\
13000\end{array}$ & $\begin{array}{r}182000 \\
343300\end{array}$ & $\begin{array}{l}25700000 \\
4690000\end{array}$ & $\begin{array}{l}425000 \\
63000\end{array}$ & $\begin{array}{l}0.257919 \\
0.33216\end{array}$ & $\begin{array}{l}0.088281 \\
0.098510\end{array}$ & $\begin{array}{l}0.103860 \\
0.108280\end{array}$ & $\begin{array}{l}0.031504 \\
0.027925\end{array}$ & $\begin{array}{l}0.444468 \\
0.381829\end{array}$ & $\begin{array}{l}0.073568 \\
0.051298\end{array}$ & $\begin{array}{l}0.70 \\
0.66\end{array}$ & $\begin{array}{l}1.90 \\
232\end{array}$ & 9.96 & 63 \\
\hline & $\begin{array}{l}70.52 \\
70.68\end{array}$ & $\begin{array}{l}4080000 \\
637000\end{array}$ & $\begin{array}{l}2110000 \\
200000\end{array}$ & $\begin{array}{l}133000 \\
264000\end{array}$ & $\begin{array}{l}343300 \\
75100\end{array}$ & $\begin{array}{l}4499000 \\
1310000\end{array}$ & 00000 & $\begin{array}{l}0.3323166 \\
0.236267\end{array}$ & $\begin{array}{l}0.0 \\
0.0\end{array}$ & $\begin{array}{l}280 \\
919\end{array}$ & $\begin{array}{l}925 \\
855 \\
855\end{array}$ & 887 & & $\begin{array}{l}0.06 \\
0.73\end{array}$ & $\begin{array}{l}2.32 \\
1.31\end{array}$ & & \\
\hline A A ARP & $\begin{array}{l}70.70 \\
70.71\end{array}$ & $\begin{array}{l}6370000 \\
739000\end{array}$ & 212000 & $\begin{array}{l}2664000 \\
254000\end{array}$ & $\begin{array}{l}571000 \\
71900\end{array}$ & $\begin{array}{l}13100000 \\
1030000\end{array}$ & 210000 & $\begin{array}{l}.2336267 \\
0.307251\end{array}$ & 0.08814 & $\begin{array}{l}0.0997919 \\
0.195605\end{array}$ & $\begin{array}{l}0.0277555 \\
0.029894\end{array}$ & $\begin{array}{l}0.485887 \\
0.428239\end{array}$ & $\begin{array}{l}0.077890 \\
0.040870\end{array}$ & 然 & $\begin{array}{l}1.31 \\
2.24\end{array}$ & & 96 \\
\hline UARRY & 70.79 & 31300 & 78500 & 74500 & 26700 & 437000 & 51100 & 0.044772 & 0.112287 & 0.106566 & 0.038192 & 0.625089 & 0.073094 & 0.66 & 2.40 & 16.07 & $\begin{array}{l}8.044 \\
8.90\end{array}$ \\
\hline & 70.83 & & 60100 & 62500 & 22900 & $\begin{array}{l}389000 \\
11500\end{array}$ & 49300 & 0.2 & 0.074216 & 0.077180 & 3279 & 0.480366 & 0.060879 & 0.69 & 68 & 19.24 & 45 \\
\hline & $\begin{array}{l}70.89 \\
70.90\end{array}$ & $\begin{array}{l}804000 \\
398000\end{array}$ & $\begin{array}{r}22800 \\
122000\end{array}$ & $\begin{array}{r}19200 \\
112000\end{array}$ & $\begin{array}{l}10100 \\
40700\end{array}$ & $\begin{array}{l}175000 \\
703000\end{array}$ & $\begin{array}{l}15100 \\
84400\end{array}$ & $\begin{array}{l}0.249255 \\
0.27225\end{array}$ & $\begin{array}{l}0.070676 \\
0.924252\end{array}$ & $\begin{array}{l}0.059516 \\
0.07795\end{array}$ & 0.031308 & $\begin{array}{l}0.542467 \\
0.48717\end{array}$ & 0.046807 & 0.66 & & 19.19 & 45 \\
\hline & $\begin{array}{l}0.900 \\
70.98\end{array}$ & $\begin{array}{l}3980000 \\
787000\end{array}$ & $\begin{array}{l}1222000 \\
256000\end{array}$ & $\begin{array}{l}1144000 \\
250000\end{array}$ & $\begin{array}{l}407000 \\
83600\end{array}$ & $\begin{array}{l}72300000 \\
1250000\end{array}$ & $\begin{array}{r}84700 \\
224000\end{array}$ & $\begin{array}{l}0.27272155 \\
0.27682\end{array}$ & $\begin{array}{l}0.0883425 \\
0.089806\end{array}$ & $\begin{array}{l}0.0777544 \\
0.087701\end{array}$ & $\begin{array}{l}0.0278311 \\
0.029327\end{array}$ & $\begin{array}{l}0.4880717 \\
0.43854\end{array}$ & $\begin{array}{l}0.057918 \\
0.078580\end{array}$ & $\begin{array}{l}0.06 \\
0.69 \\
0.69\end{array}$ & $\begin{array}{l}1.66 \\
1.99\end{array}$ & $\begin{array}{l}\begin{array}{l}9.19 \\
17.91\end{array}\end{array}$ & $\begin{array}{l}44 \\
77\end{array}$ \\
\hline UARRY & 71.11 & 275000 & 86600 & 91500 & ${ }_{30900}$ & 539000 & 75500 & $\begin{array}{l}0.27008 \\
0.250341\end{array}$ & $\begin{array}{l}0.068500 \\
0.078835\end{array}$ & 0.083295 & $\begin{array}{l}0.028 \\
0.028129\end{array}$ & $\begin{array}{l}0.4358004 \\
0.49069\end{array}$ & 0.068730 & $\begin{array}{l}0.09 \\
0.70\end{array}$ & $\begin{array}{l}1.999 \\
1.47\end{array}$ & 20.25 & 26 \\
\hline & 71.19 & 5 & 140000 & 148000 & 51700 & 824000 & 104000 & 0.263693 & 0.081315 & 0.085962 & 0.030028 & 0.478597 & 0.060405 & 0.68 & 1.85 & 18.78 & 8 \\
\hline & $\begin{array}{l}71.20 \\
71.23\end{array}$ & $\begin{array}{l}613000 \\
11300\end{array}$ & $\begin{array}{r}173000 \\
35600\end{array}$ & $\begin{array}{r}175000 \\
335700\end{array}$ & $\begin{array}{r}57400 \\
12200\end{array}$ & $\begin{array}{l}924000 \\
196000\end{array}$ & 9000 & $\begin{array}{l}0.295935 \\
028189\end{array}$ & $\begin{array}{l}0.083518 \\
0.08892060\end{array}$ & $\begin{array}{l}0.084484 \\
0.085464\end{array}$ & 0.027711 & 0.446075 & 62277 & 0. & 83 & 18.67 & 59 \\
\hline & $\begin{array}{l}71.23 \\
71.24\end{array}$ & $\begin{array}{l}121000 \\
562000\end{array}$ & $\begin{array}{l}356000 \\
151000\end{array}$ & $\begin{array}{l}367000 \\
150000\end{array}$ & $\begin{array}{l}122200 \\
47200\end{array}$ & $\begin{array}{r}1960000 \\
721000\end{array}$ & $\begin{array}{l}277000 \\
97800\end{array}$ & $\begin{array}{l}0.281789 \\
0.325043\end{array}$ & $\begin{array}{l}0.0882906 \\
0.08734\end{array}$ & $\begin{array}{l}0.0855688 \\
0.086755\end{array}$ & $\begin{array}{l}0.028412 \\
0.027299\end{array}$ & $\begin{array}{l}0.456451 \\
04417004\end{array}$ & 0 & & $\begin{array}{l}1.197 \\
1.93\end{array}$ & 12 & 51 \\
\hline RY & & $\begin{array}{l}582000 \\
38700\end{array}$ & ${ }_{9450}$ & 8620 & 3590 & 60400 & 8340 & $\begin{array}{l}0.299768 \\
0.097\end{array}$ & & 0.066770 & 7808 & 0.467854 & & & & & 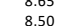 \\
\hline & 71.43 & 543000 & 170000 & 232000 & 75900 & 1170000 & $\begin{array}{l}0 \\
1990000\end{array}$ & 0.227206 & 0.071133 & $\begin{array}{l}0.0977075 \\
0\end{array}$ & 0.031759 & $\begin{array}{l}0.48950 \\
0.485\end{array}$ & 0.083267 & 0.7 & $i_{0}$ & & 39 \\
\hline QUARRY & 71.69 & 440000 & 135000 & 139000 & 47600 & 755000 & 103000 & 0.271672 & 0.083354 & 0.085824 & 0.029390 & 0.466164 & 0.063596 & 0.68 & 1.84 & 18.57 & 66 \\
\hline & 71.69 & & & & 32800 & & 0 & & & & & & & 0.67 & & & \\
\hline YY & $\begin{array}{l}71.84 \\
73227\end{array}$ & $\begin{array}{l}378000 \\
339400\end{array}$ & $\begin{array}{l}113000 \\
1116000\end{array}$ & $\begin{array}{l}113000 \\
1106000\end{array}$ & $\begin{array}{l}33000 \\
390900\end{array}$ & $\begin{array}{l}520000 \\
583000\end{array}$ & $\begin{array}{l}94700 \\
45000\end{array}$ & $\begin{array}{l}0.301989 \\
0.3044\end{array}$ & $\begin{array}{l}0.090277 \\
0.098627\end{array}$ & $\begin{array}{l}0.090277 \\
0.089624\end{array}$ & 0.026364 & $\begin{array}{l}0.415435 \\
0.45434\end{array}$ & $\begin{array}{l}0.075657 \\
0.035077\end{array}$ & $\begin{array}{l}0.68 \\
0.638\end{array}$ & $\begin{array}{l}1.83 \\
2,12\end{array}$ & 18.91 & $\begin{array}{l}8.57 \\
8.83\end{array}$ \\
\hline QUA & $\begin{array}{l}7.271 \\
73.70\end{array}$ & $\begin{array}{l}39 \\
57\end{array}$ & 158000 & $\begin{array}{l}1100000 \\
14100\end{array}$ & $\begin{array}{l}399500 \\
7530\end{array}$ & $\begin{array}{l}5838000 \\
127000\end{array}$ & $\begin{array}{l}45500 \\
11500\end{array}$ & $\begin{array}{l}0.3 \\
0.2\end{array}$ & $\begin{array}{l}0.0860642 \\
0.067686\end{array}$ & $\begin{array}{l}0.0809624 \\
0.060404\end{array}$ & $\begin{array}{l}858 \\
258\end{array}$ & $\begin{array}{l}431 \\
060\end{array}$ & $\begin{array}{l}0.035 \\
0.049265\end{array}$ & $\begin{array}{l}0.63 \\
0.68\end{array}$ & $\begin{array}{l}2.12 \\
1.39\end{array}$ & $\begin{array}{l}1,1.08 \\
19.71\end{array}$ & 28 \\
\hline QUA & 74.14 & 38000 & 12900 & 14500 & 3560 & 73800 & 8420 & 0.251356 & 0.085329 & 0.095912 & 0.023548 & 0.488160 & 0.055695 & 0.67 & 1.08 & 24.10 & 19 \\
\hline & & & & & & & & 0.2 & & & & & & 年 & & & 8.49 \\
\hline nou & 124 & $\begin{array}{l}17110000 \\
868000\end{array}$ & $\begin{array}{r}471000 \\
144000\end{array}$ & $\begin{array}{l}\begin{array}{r}5566000 \\
122000\end{array} \\
0\end{array}$ & $\begin{array}{l}1430000 \\
319000\end{array}$ & $\begin{array}{l}388 \\
102\end{array}$ & $\begin{array}{l}2560000 \\
45800\end{array}$ & & $\begin{array}{l}0.068103 \\
0.0645525\end{array}$ & $\begin{array}{l}0.065934 \\
0.05466\end{array}$ & & & $\begin{array}{l}0.037016 \\
0.025052\end{array}$ & $\begin{array}{l}0.6 \\
0.5\end{array}$ & & & $\begin{array}{l}4.13 \\
40.05\end{array}$ \\
\hline & & $\begin{array}{l}86 \\
42\end{array}$ & $\begin{array}{l}1444000 \\
982200\end{array}$ & $\begin{array}{l}{ }_{9}^{21222000} \\
966000\end{array}$ & 300 & $\begin{array}{l}10200000 \\
959000\end{array}$ & $\begin{array}{l}455800 \\
63900\end{array}$ & 0.2584643 & $\begin{array}{l}0.0605425 \\
0.058838\end{array}$ & & 0.0 & $\begin{array}{l}0.45 \\
0.574591\end{array}$ & 0.0 & 0. & & & 56 \\
\hline & & & & 175000 & 65300 & 1510 & 89800 & & 0.066260 & 0.066260 & & & & 0. & & & 22 \\
\hline & & & 11 & 110 & 34500 & 94 & 61900 & & 0.06 & 0.0 & & & & 0. & 0 & 30 & 04 \\
\hline & $\begin{array}{l}125 \\
125\end{array}$ & $\begin{array}{l}129000 \\
7711000\end{array}$ & $\begin{array}{l}14100 \\
132000\end{array}$ & 13800 & $\begin{array}{l}4180 \\
34400\end{array}$ & $\begin{array}{r}1880000 \\
1200000\end{array}$ & $\begin{array}{l}10500 \\
56200\end{array}$ & 0.358752 & 0.039212 & $\begin{array}{l}0.038378 \\
0.054020\end{array}$ & 0.0 & $\begin{array}{l}0.522832 \\
0.53153\end{array}$ & 0.029201 & 0 & & 21 & 15 \\
\hline & & & & & & & & & & & & & & & & & $\begin{array}{l}3.65 \\
3.47\end{array}$ \\
\hline 然 & $\begin{array}{l}125.5 \\
125.4\end{array}$ & $\begin{array}{l}5084 \\
304\end{array}$ & $\begin{array}{l}10.3 \\
53\end{array}$ & $\begin{array}{l}{ }_{43500} \\
43220\end{array}$ & $\begin{array}{l}12000 \\
10700\end{array}$ & $\begin{array}{l}8930000 \\
3930\end{array}$ & $\begin{array}{l}46200 \\
16200\end{array}$ & $\begin{array}{l}0.38 \\
0.37\end{array}$ & $\begin{array}{l}0.0653510 \\
0.063\end{array}$ & $\begin{array}{l}0.052828 \\
0.0528\end{array}$ & & 860 & $\begin{array}{l}0.02919 \\
0.0193\end{array}$ & 0.6 & & & $\begin{array}{l}5.47 \\
3.61\end{array}$ \\
\hline RIVET & 93 & 1910000 & 570000 & 1160000 & 685000 & 221000000 & 3070000 & 0.064757 & 0.019325 & 0.039329 & 224 & 0.749280 & 0.104085 & 0.9 & & & 8.21 \\
\hline & & & & & & & & & & & & & & & & & 20 \\
\hline & & & & & & & & & & & & & & & & & ${ }_{10}$ \\
\hline & $\begin{array}{l}93.28 \\
93.33\end{array}$ & 00 & $\begin{array}{l}68 \\
31\end{array}$ & $\begin{array}{l}13600000 \\
678000\end{array}$ & 777000 & 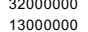 & $\begin{array}{l}\begin{array}{l}66600000 \\
2060000\end{array}\end{array}$ & $\begin{array}{l}0.068652 \\
0.049581\end{array}$ & $\begin{array}{l}0.0116666 \\
0.017972\end{array}$ & $\begin{array}{l}0.0 \\
0.0\end{array}$ & $\begin{array}{l}0.0 \\
0.0\end{array}$ & 313 & ${ }_{41}^{37}$ & & & & $\begin{array}{l}8.43 \\
836\end{array}$ \\
\hline SRIV & 列3 & 80 & 31 & 21 & 00 & 371 & 000 & 0.0 & 0.020158 & 0.0 & 0.0 & 02 & 0.1 & 0. & 49 & $\begin{array}{l}19.25 \\
19.55\end{array}$ & $\begin{array}{l}8.36 \\
8.28\end{array}$ \\
\hline RIVE & 93.42 & 1860000 & 679000 & 1570000 & 930000 & 28100000 & 0000 & 0.0 & 0.018185 & 0.0 & 007 & 0.7 & 33 & 0.9 & & & 34 \\
\hline & & & & & & & & & & & & & & & & & \\
\hline & & & & & & $\begin{array}{l}466000000 \\
{ }_{3} 5700\end{array}$ & $\begin{array}{l}73600000 \\
75600\end{array}$ & & & & & & & & & & 62 \\
\hline & & & & & 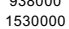 & 00 & 00 & & & & & & & & & & 57 \\
\hline & & & & & 528000 & 192 & & 0.05 & 0.0 & 0.0 & & & & & & & $\begin{array}{l}8.005 \\
8.52\end{array}$ \\
\hline & & & & & & & & & & & & & & & & & \\
\hline & & & & & $\begin{array}{l}13600000 \\
7255000\end{array}$ & $\begin{array}{l}503 \\
243\end{array}$ & & 0.044900 & & & & & & & & & 57 \\
\hline & & & & & & & & & & & & & & & & & 50 \\
\hline & & & & & 0000 & 00 & 年 & 0.0 & & & & & 3605 & & & & 74 \\
\hline & & & & 10000 & bo & 4900000 & 50000 & 0.046979 & 0.0 & 0.0 & & 54 & & & & & 55 \\
\hline & & & & & & & & 0.0 & .009937 & 0.025596 & & & & & & & \\
\hline & & & & & & & & & & & & & & & & & \\
\hline & & & & & & & & & & & & & & & & & \\
\hline & & & & & & & & & & & & & & & & & \\
\hline & & & & & & & & & & & & & & 0.91 & 1.52 & 9.13 & .39 \\
\hline
\end{tabular}


Cretaceous dataset for OPTiMAL

$\begin{array}{llllllllllllll}\text { AGE (GTS 2012) } & 1302 & 1300 & 1298 & 1296 & 1292 & 1292^{\prime} & \text { Fr.1302 } & \text { Fr.1300 } & \text { Fr.1298 } & \text { Fr.1296 } & \text { Fr.1292 } & \text { Fr.1292' } & \text { TEx86 }\end{array}$

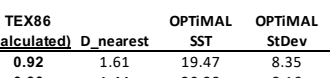

BASS RIVER
BASS RVER
BASS RIVER
BASS RIVER

BAAS RIVER
BASS RIVR

BASS RIVER
BASS RIVER
BASS RIVER

BASS RIVER
BASS RIVER

BASS RIVR
BASS RIVER

BASS RIVER
BASS RIVER

BASS RIVER
BASS RIVER
BASS RIVER

BASS RIVRR
BASS RIVER

BASS RVRR
BASS RIVR
BASS

BASS RIVER
BASS RIVER

BASS RER
BASS RIVER

BAAS RIVR
BASS PEVR

BASS RIVR
BASS RIVER

BASS RIVER
BASS RIVER
BASS RVER

BAAS RIVER
BASS RIVER

BASS RIVER
BASS RIVER

BASS RIVER
BASS RIVER

BASS RIVRR
BASS RIVER

Bass
BASS RIVR
BASS RIVR

BAAS RIVR
BASS RIVER

BASS RIVER
BASS RIVER
BASS RIVER

BASS RIVR
BASS RIVER

BASS RIVR
BASS RIVER

BASS RIVER
BASS RIVER
BASS RIVER

BASS RVVR
BASS RIVER

BASS RIVER
BASS RIVER

BAASS RVER
BASS RIVR

WUNSTORF
WUNSTORF
WUNSTORF

WUNSTORF
WUNSTORF
WUST

WUNSTORF
WUNSTORF

WUNSTORF
WUNSTORF
WUNSTORF

WUNSTORF
WUNSTORF
WUSTOPF

WUNSTORF

WUNSTORF

WUNSTORF

WUNSTORF
WUNSTORF
WUNSTOPF

WUNSTORF

WUNSTORF

WUNSTORF

WUNSTORF
WUNSTORF
WUNSTORF

WUSSTORF

WUNSTRRF \begin{tabular}{cccccc}
1302 & 1300 & 1298 & 1296 & 1292 & 1292 \\
GDGTO & GDGT1 & GDGT2 & GDGT3 & CREN & CREN \\
\hline & CR &
\end{tabular}

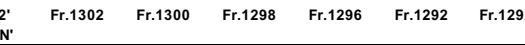
0.92
0.90
0.92

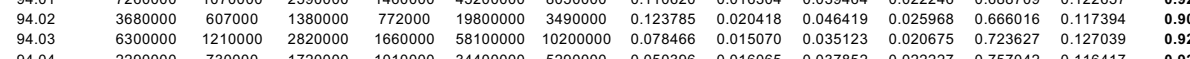

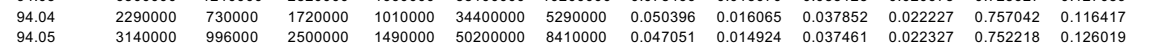

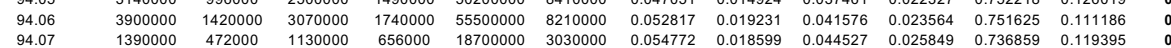

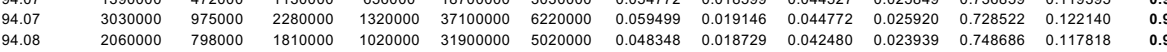

$\begin{array}{lllllllllllll}94.09 & 4150000 & 15200000 & 3330000 & 1980000 & 63400000 & 9570000 & 0.049434 & 0.018106 & 0.039666 & 0.023585 & 0.755211 & 0.113996 \\ 94.10 & 2350000 & 1020000 & 2590000 & 1560000 & 44600000 & 7620000 & 0.039337 & 0.017074 & 0.043355 & 0.026113 & 0.746568 & 0.127553\end{array}$

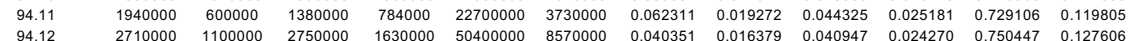

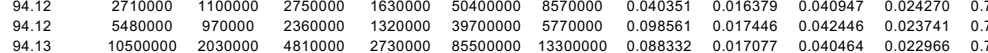

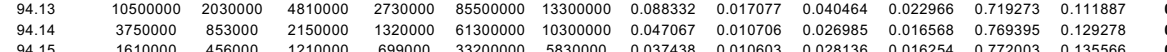

$\begin{array}{llllllllllllll}94.15 & 1610000 & 456000 & 1210000 & 699000 & 33200000 & 58300000 & 0.037438 & 0.010603 & 0.028136 & 0.016254 & 0.772003 & 0.135566 & 0.94 \\ 94.16 & 2450000 & 727000 & 1830000 & 1070000 & 44900000 & 6730000 & 0.042456 & 0.012598 & 0.031712 & 0.018542 & 0.778069 & 0.116624 & 0.93\end{array}$

$\begin{array}{lllllllllllll}94.17 & 4870000 & 1680000 & 4230000 & 2570000 & 1070000000 & 17400000 & 0.035354 & 0.012196 & 0.030708 & 0.018657 & 0.776770 & 0.126316 \\ 94.18 & 4630000 & 649000 & 1690000 & 908000 & 36800000 & 6160000 & 0.091075 & 0.012766 & 0.033244 & 0.017861 & 0.723882 & 0.121172\end{array}$

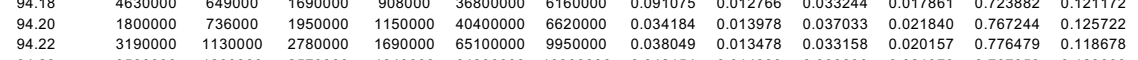

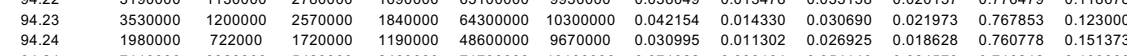

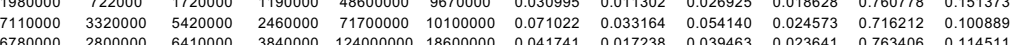

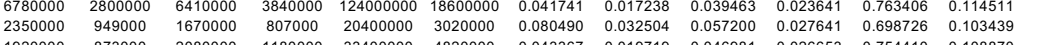

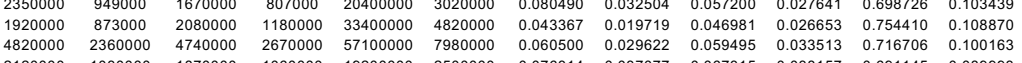

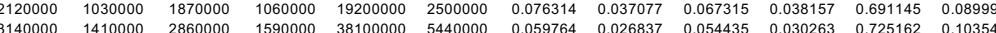

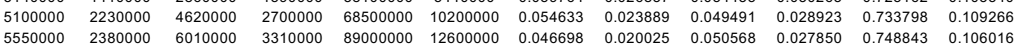

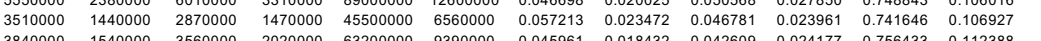
$\begin{array}{lllllllllllll}3840000 & 1540000 & 3560000 & 2020000 & 632000000 & 93900000 & 0.045961 & 0.018432 & 0.042609 & 0.024177 & 0.756433 & 0.112388\end{array}$

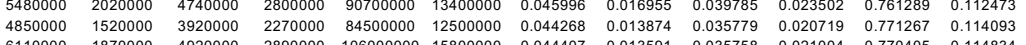
$\begin{array}{lllllllllllll}6110000 & 1870000 & 4920000 & 2890000 & 1060000000 & 15800000 & 0.044407 & 0.013591 & 0.035758 & 0.021004 & 0.770405 & 0.114834 \\ 220000 & 722000 & 190000 & 110000 & 4600000 & 792000 & 0 & 0\end{array}$ $\begin{array}{llllllllllll}2280000 & 7222000 & 19000000 & 1110000 & 46300000 & 7920000 & 0.037854 & 0.011987 & 0.031545 & 0.018429 & 0.768694 & 0.131492 \\ 2050000 & 699000 & 1650000 & 1000000 & 36800000 & 5920000 & 0.0426263 & 0.014526 & 0.034290 & 0.020782 & 0.764771 & 0.123020\end{array}$

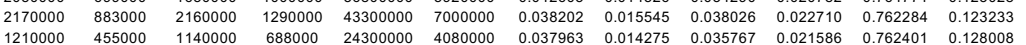

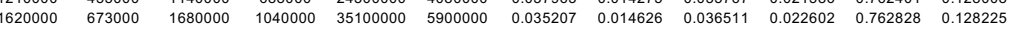

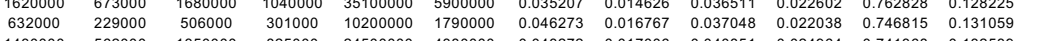

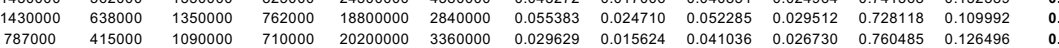
$\begin{array}{lllllllllllll}2050000 & 556000 & 1210000 & 711000 & 20900000 & 3620000 & 0.070575 & 0.019141 & 0.041657 & 0.024478 & 0.719524 & 0.124626 & 0\end{array}$ $\begin{array}{lllllllllllllll}810000 & 354000 & 801000 & 503000 & 13700000 & 2120000 & 0.044291 & 0.019357 & 0.043799 & 0.027504 & 0.749125 & 0.115923 & 0\end{array}$ $\begin{array}{llllllllllll}1844000 & 59100 & 99900 & 58200 & 1280000 & 179000 & 0.098914 & 0.031771 & 0.053704 & 0.031287 & 0.688098 & 0.096226\end{array}$

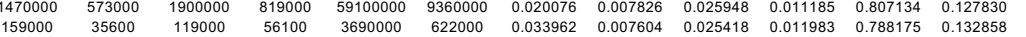
$\begin{array}{llllllllllll}415000 & 123000 & 306000 & 137000 & 86600000 & 1270000 & 0.038035 & 0.011273 & 0.028045 & 0.012556 & 0.793694 & 0.1163996\end{array}$

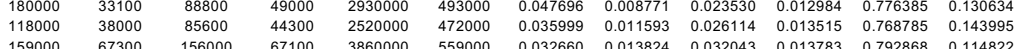
$\begin{array}{cccccccccccc}159000 & 67300 & 156000 & 67100 & 3860000 & 559000 & 0.032660 & 0.013824 & 0.032043 & 0.013783 & 0.792868 & 0.114822 \\ 2800000 & 813000 & 2240000 & 1050000 & 78000000 & 11800000 & 0.028955 & 0.008447 & 0.023164 & 0.010858 & 0.806593 & 0.122023\end{array}$ $\begin{array}{lllllllllllll}1040000 & 354000 & 9044000 & 430000 & 26100000 & 37400000 & 0.031933 & 0.0108770 & 0.027757 & 0.013203 & 0.801400 & 0.114837\end{array}$ $\begin{array}{llllllllllll}2790000 & 976000 & 27110000 & 1220000 & 75500000 & 12800000 & 0.028764 & 0.010062 & 0.027939 & 0.012578 & 0.788692 & 0.131964 \\ 1910000 & 722000 & 1960000 & 890000 & 55000000 & 7900000 & 0.027931 & 0.010558 & 0.028663 & 0.013015 & 0.804305 & 0.115527\end{array}$ $\begin{array}{lllllllllllll}4000000 & 1670000 & 4510000 & 1940000 & 96100000 & 16100000 & 0.032175 & 0.013433 & 0.036277 & 0.015605 & 0.773005 & 0.129505\end{array}$

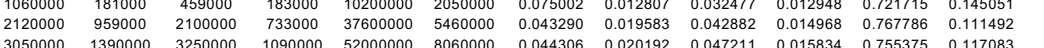
$\begin{array}{llllllllllll}6170000 & 2730000 & 6100000 & 2130000 & 103000000 & 16300000 & 0.045225 & 0.020010 & 0.044712 & 0.015612 & 0.754966 & 0.119475 \\ 444000 & 146000 & 391000 & 233000 & 12400000 & 2230000 & 0.028023 & 0.009215 & 0.024678 & 0.014706 & 0.782631 & 0.140747\end{array}$ $\begin{array}{llllllllllllll}1510000 & 312000 & 825000 & 410000 & 239000000 & 4410000 & 0.048140 & 0.009947 & 0.026302 & 0.013071 & 0.761947 & 0.140594\end{array}$

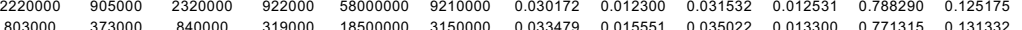

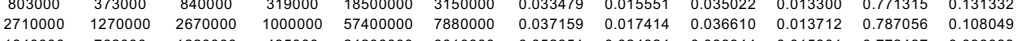

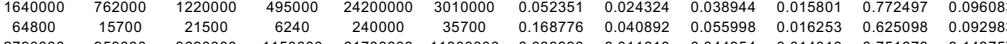

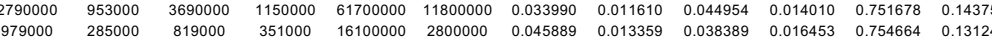
$\begin{array}{llllllllllll}513000 & 138000 & 541000 & 243000 & 16800000 & 2950000 & 0.024215 & 0.006514 & 0.025537 & 0.011470 & 0.793014 & 0.139249 \\ 105000 & 29550 & 80100 & 35400 & 1820000 & 360000 & 0.043210 & 0.012140 & 0.032963 & 0.014568 & 0.748971 & 0.148148\end{array}$ 
Cretaceous dataset for OPTiMAL

$\begin{array}{llllllllllllll}\text { AGE (GTS 2012) } & 1302 & 1300 & 1298 & 1296 & 1292 & 1292^{\prime} & \text { Fr.1302 } & \text { Fr.1300 } & \text { Fr.1298 } & \text { Fr.1296 } & \text { Fr.1292 } & \text { Fr.1292' } & \text { TEx } 8\end{array}$

B

BRAZOS RIV
BRAZOS RIVE
BRAZOS RIV
BRAZOS

BRAZOS RIVER
BRAZOS RIVER
BRAZOS RIVER

BRAZOS RIVER
BRAZOS RIVER
BRAZOS RIVER

BRAZOS RIVER

BRAZOS RIVER

BRAZOS RIVR

BRAOSO RRVR
BRAZOS RIVER

BRAZOS RIVE

BRAZOS RIVER

BRAZOS RIVER

BRAZOS RIVER
BRAZOS RIVER
BRAZOS RIVER

BRAZOS RIVER
BRAZOS RIVER
B

BRAZOS RIVER
BRAZOS RIVER

BRAZOS RIVER

BRAOS REVE
BRAZOS RIVER

BRAZOS RIVE

BRAZOS RIVE

BRAZOS RIVER

BRZZOS RIVER
BRAZOS RIVER

BRAZOS RIVER
BRAZOS RIVER

BRAZOS RIVER
BRAZOS RIVER
BRAZOS RIVER

BRAZOS RIVER
BRAZOS RIVER

BRZOOS RIER

BRAZOS RIVER

BRAZOS RIVER
BRAZOS RIVER
G

BRAZOS RIVER
BRAZOS RIVER

BRAZOS RIVER
BRAZOS RIVER

BRAZOS RIVER
BRAZOS RIVER

BRAZOS RIVER
BRAZOS RIVER

BRAZOS RIVER
MEIRS FARM 1

MEIRS FARM 1
MEIRS FARM 1

MEIRS FARM

MEIRS FARM

MERS ARRM 1

DSDP 398

DSDP 398

DSSPP 398
DSP 398

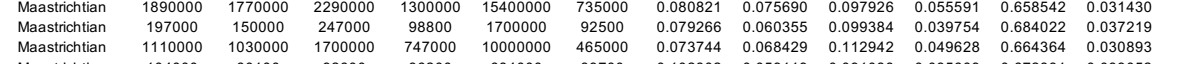

$\begin{array}{llllllllllllll}\text { Maastrichtian } & 104000 & 60100 & 92600 & 36200 & 684000 & 39700 & 0.102302 & 0.059119 & 0.091088 & 0.035609 & 0.672831 & 0.039052 \\ \text { Maastrichtian } & 84900 & 36200 & 57400 & 263300 & 623000 & 54100 & 0.096269 & 0.041048 & 0.065087 & 0.029822 & 0.706429 & 0.061345\end{array}$

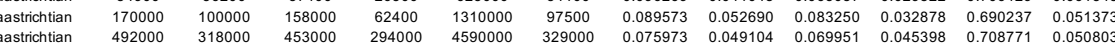

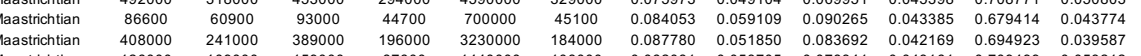

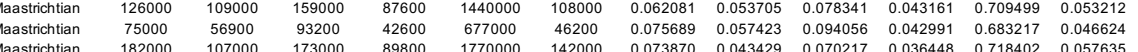

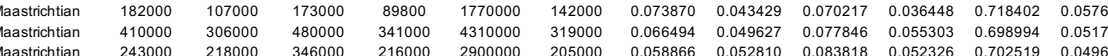

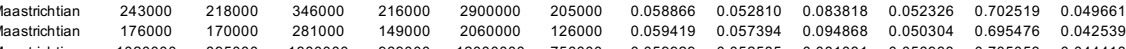

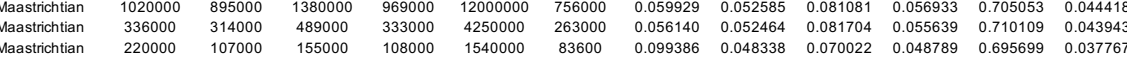

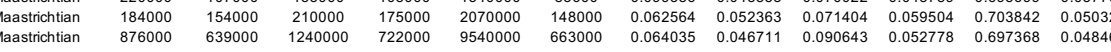

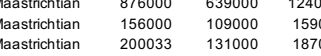

\begin{tabular}{llllll} 
& 131000 & 187000 & 102000 & 1730000 \\
\hline
\end{tabular}

$\begin{array}{llll}\text { Maastrichtian } & 228000 & 169000 & 236000\end{array}$

\begin{tabular}{lll} 
& 425000 & 4730000 \\
25000 & 2610000 \\
\hline
\end{tabular}

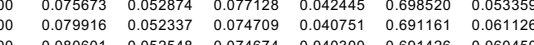

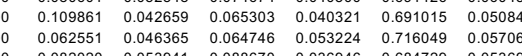

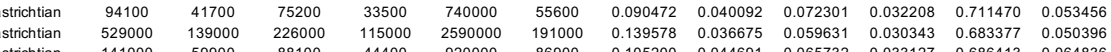

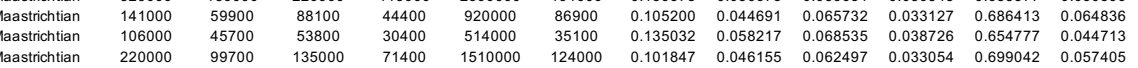

Maastrichtian $\quad 657000-99700 \quad 135000$

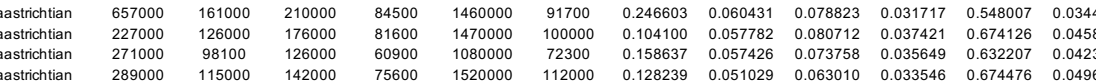

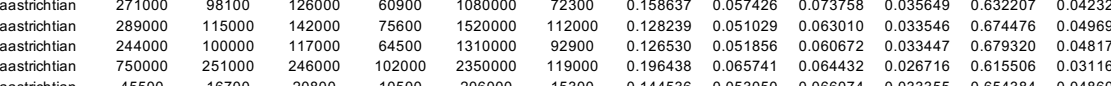

$\begin{array}{llll} & 45500 & 16700 & 20000 \\ \text { Mastrichtian } & 75500 & 22100 & 24900\end{array}$

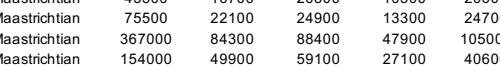

$\begin{array}{llllllll}0.144536 & 0.053050 & 0.066074 & 0.033355 & 0.655306 & 0.03116\end{array}$

$\begin{array}{lllllll}0.188609 & 0.055209 & 0.062203 & 0.033225 & 0.617037 & 0.043717\end{array}$

$\begin{array}{llllll}0.211568 & 0.068553 & 0.081192 & 0.037230 & 0.557769 & 0.04368 \\ 0.133181 & 0.054810 & 0.064136 & 0.034522 & 0.660995 & 0.05235\end{array}$

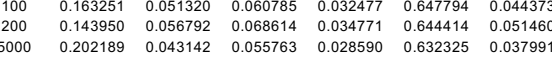

$\begin{array}{lllllll} & 0.177356 & 0.050277 & 0.060174 & 0.030087 & 0.637371 & 0.044735 \\ 0.0130030 & 0.061862 & 0.085285 & 0.035736 & 0.639640 & 0.047428\end{array}$

$\begin{array}{llllll}0.148158 & 0.061894 & 0.071864 & 0.039047 & 0.632789 & 0.04622 \\ 0.180451 & 0.062155 & 0.074060 & 0.034336 & 0.609023 & 0.03997 \\ & 0415 & 0.02515 & 0.0178 & 0\end{array}$

$\begin{array}{lllllll} & 0.471157 & 0.025115 & 0.017781 & 0.008481 & 0.467060 & 0.01040 \\ & 0.127505 & 0.058835 & 0.068205 & 0.035519 & 0.659785 & 0.055151\end{array}$

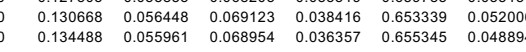

$\begin{array}{lllllll}0.120937 & 0.054054 & 0.069620 & 0.040712 & 0.656859 & 0.0578 \\ 0.196383 & 0.053145 & 0.070121 & 0.021524 & 0.621325 & 0.03750\end{array}$

$\begin{array}{llllllll}0.000 & 161000 & 0.174066 & 0.075796 & 0.093864 & 0.046932 & 0.591605 & 0.017737 \\ 000 & 41300 & 0.132302 & 0.050188 & 0.060923 & 0.031228 & 0.6667782 & 0.055777 \\ 000 & 121000 & 0.180028 & 0.040962 & 0.051289 & 0.028605 & 0.657464 & 0.041651\end{array}$

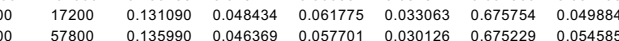

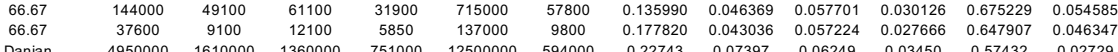

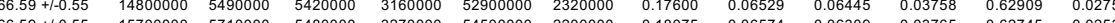

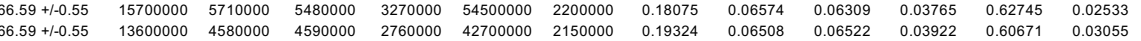
$\begin{array}{lllllllllllll}66.59+-1.55 & 15500000 & 6030000 & 6920000 & 3950000 & 56200000 & 3630000 & 0.16806 & 0.06538 & 0.07503 & 0.04283 & 0.60935 & 0.03936 \\ 6.59+1-0.55 & 27800000 & 9390000 & 8860000 & 5250000 & 79700000 & 3720000 & 0.20635 & 0.06970 & 0.06577 & 0.03897 & 0.59160 & 0.0276 \\ 6 & & & & \end{array}$

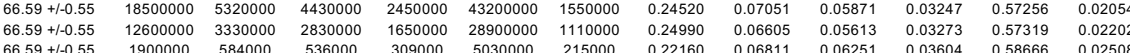

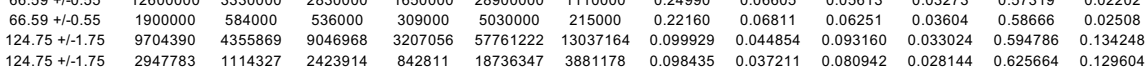

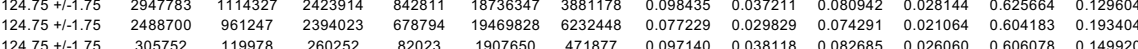

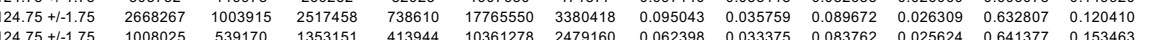

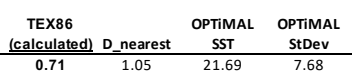

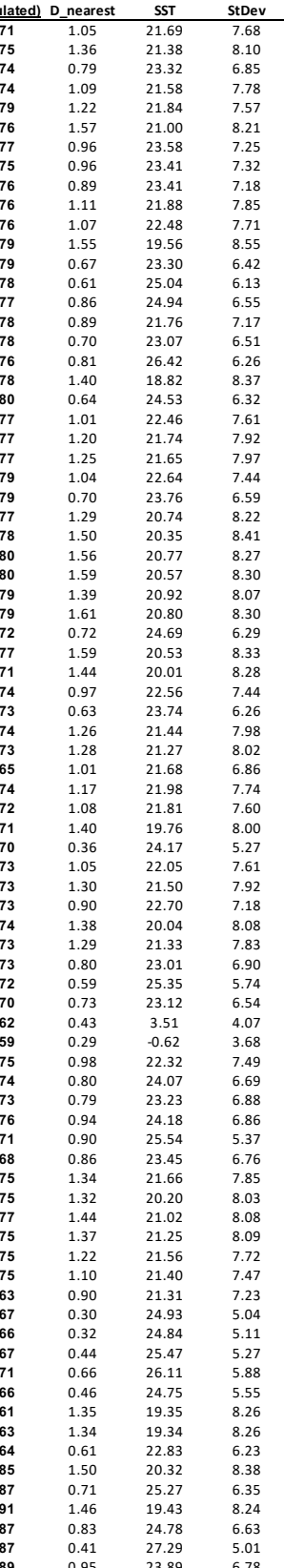


Cretaceous dataset for OPTiMAL

$\begin{array}{llllllllllllll}\text { AGE (GTS 2012) } & 1302 & 1300 & 1298 & 1296 & 1292 & 1292^{\prime} & \text { Fr.1302 } & \text { Fr.1300 } & \text { Fr.1298 } & \text { Fr.1296 } & \text { Fr.1292 } & \text { Fr.1292 } & \text { TEXof }\end{array}$ $\begin{array}{lll}\text { TEX86 } & \text { OPTTMAL } & \text { OPTTMAL } \\ \text { StDev }\end{array}$

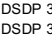
$\operatorname{DSDP} 39$

DSDP 398
DSDP 398

DSDP 398
DSDP 398

$\operatorname{DSDP} 398$
DSDP 3998

DSDP 398
DSD

DSDP 398
DSDP 398

DSD 398
DSDP 398
DSD 398

DSD $P 398$
DSDP 398

DSDP 398
DSD 398
DSD P P
DSDP 398
SH 398

SHQUALAK

SHUQUALAK

SSHUQUALAAK
SHUUUALAK

SHUQUALAK
SHUQUALAK

SHUQUALAK
SHUQUALAK

SHUQUALAK

SHUQUALAK
SHUQUALAK
SHUQUALAK

SHHQUALAK
SHUQUALAK

SHUQUALAK
SHUQUALAK

SHUQUALAK
SHUQUALAK

SHUQUALAK

SHUQUALAK
SHUQUALAK
SHUQUALAK

SHUQUALAK
SHUQUALAK

SHUQUALAK
SHUQUALAK

SHHQUALAK
SHUQUALAK

SHUQUALAK
SHUQUALAK

SHHQUALAK
SHUQUALAK

SHUQUALAK
SHUQUALAK

SHUQUALAK
SHUQUALAK

SHUQUALAK

SHUQUALAK
SHUQUALAK

SHUQUULAK
SHUQUALAK

SHUQUALAK
SHUQUALAK

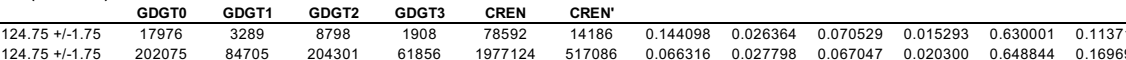

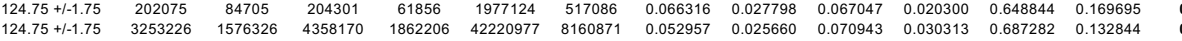

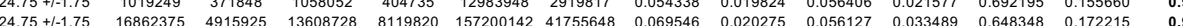

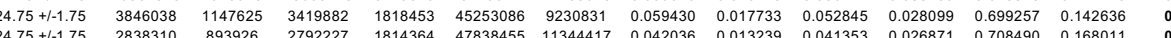

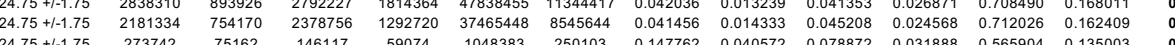
$\begin{array}{lllllllllllll}124.75+1-1.75 & 1218731 & 398839 & 1400297 & 837445 & 2047021 & 4898254 & 0.041703 & 0.013648 & 0.047916 & 0.028655 & 0.700465 & 0.167612 \\ 124.75+1-1.75 & 557985 & 240281 & 693339 & 372325 & 7694016 & 1625767 & 0.049891 & 0.021484 & 0.062021 & 0.033291 & 0.687948 & 0.145365\end{array}$

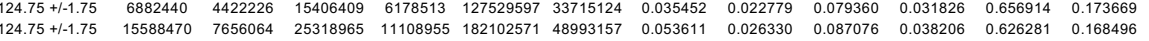

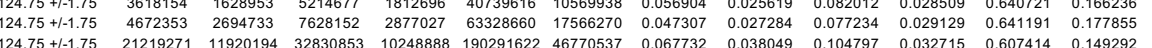

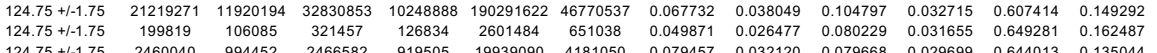
$\begin{array}{lllllllllllll}124.75+1-1.75 & 2400070 & 1221022 & 1113267 & 1229232 & 27828607 & 7403318 & 0.058260 & 0.029640 & 0.027024 & 0.029839 & 0.6755525 & 0.179712 \\ 124.75+-1.175 & 5293050 & 3327618 & 7601975 & 2243383 & 52062894 & 11064688 & 0.064871 & 0.040783 & 0.093169 & 0.027495 & 0.638076 & 0.135607\end{array}$

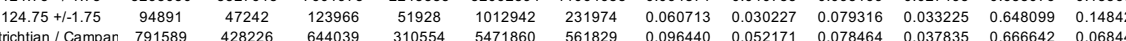

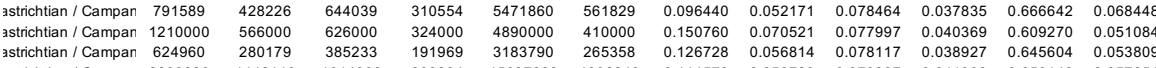

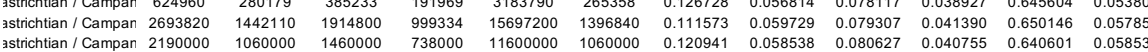

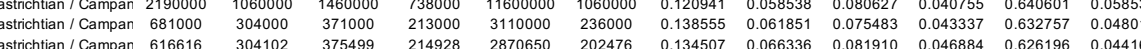

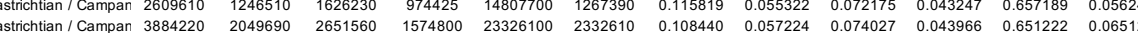

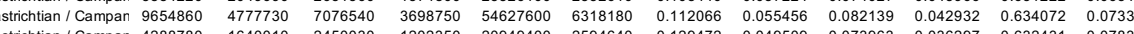
$\begin{array}{lllllllllllllll} & \end{array}$

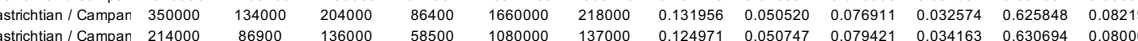

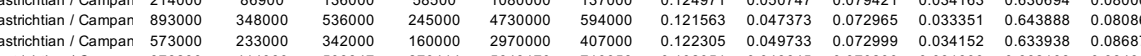

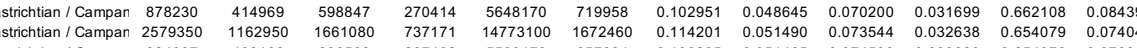
$\begin{array}{lllllllllllll}\text { asstrichtian / Campan } & 1322920 & 600212 & 780649 & 370905 & 7162190 & 764999 & 0.120245 & 0.054555 & 0.070956 & 0.033713 & 0.650997 & 0.0695\end{array}$

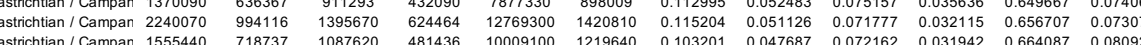
$\begin{array}{llllllllllllll} & \end{array}$

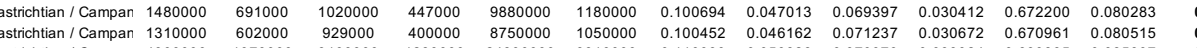

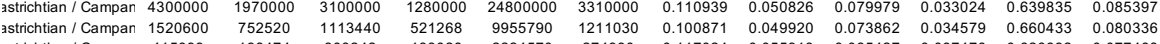

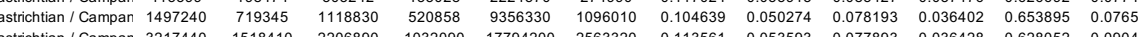

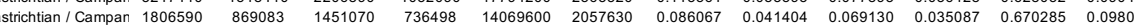

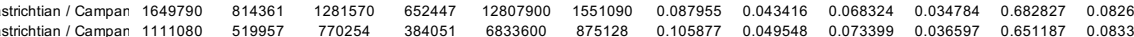

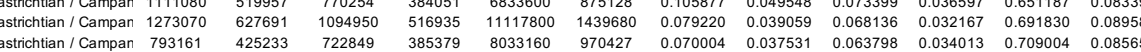
$\begin{array}{llllllllllllllll} & & & & \end{array}$

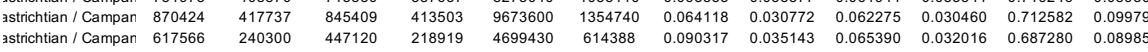
$\begin{array}{lllllllllllll}\text { asstrichtan / Campan } & 860456 & 487236 & 1033650 & 468663 & 10826500 & 1622560 & 0.056242 & 0.031847 & 0.067563 & 0.030633 & 0.707658 & 0.106050\end{array}$

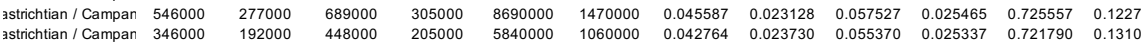

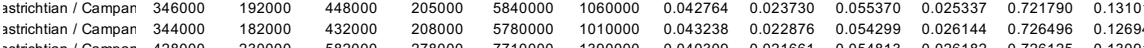
$\begin{array}{lllllllllllll}\text { astrichtian / Campan } & 4280000 & 230000 & 582000 & 278000 & 7710000 & 1390000 & 0.040309 & 0.021661 & 0.054813 & 0.026182 & 0.726125 & 0.130910 \\ \text { astrichtian /Campan } & 4054444 & 217178 & 479735 & 222243 & 5104690 & 807574 & 0.056025 & 0.030010 & 0.066290 & 0.030710 & 0.705373 & 0.111592\end{array}$

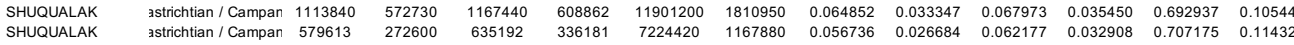

\begin{tabular}{|c|c|c|}
\hline & $\underset{\text { SST }}{\text { OPTIMAL }}$ & $\begin{array}{c}\text { OPTiMAL } \\
\text { StDev }\end{array}$ \\
\hline 1.11 & 23.70 & 7.16 \\
\hline 1.33 & 20.91 & 7.88 \\
\hline $\begin{array}{l}1.33 \\
1.43\end{array}$ & $\begin{array}{l}20.27 \\
2.19\end{array}$ & $\begin{array}{l}8.15 \\
8.12\end{array}$ \\
\hline 2.21 & 16.59 & $\begin{array}{r}8.1 .87 \\
\end{array}$ \\
\hline 1.52 & 19.15 & 8.45 \\
\hline $\begin{array}{l}1.96 \\
1.79\end{array}$ & $\begin{array}{l}17.31 \\
18.02\end{array}$ & $\begin{array}{l}8.77 \\
8.64\end{array}$ \\
\hline 1.49 & 20.97 & 8.29 \\
\hline $\begin{array}{l}1.92 \\
1.98\end{array}$ & $\begin{array}{l}17.34 \\
17.21\end{array}$ & $\begin{array}{l}8.78 \\
8.80\end{array}$ \\
\hline 1.86 & 17.54 & 8.75 \\
\hline $\begin{array}{l}2.79 \\
1.31\end{array}$ & $\begin{array}{l}15.96 \\
20.69\end{array}$ & $\begin{array}{l}8.900 \\
8.08\end{array}$ \\
\hline $\begin{array}{l}1.53 \\
1.53\end{array}$ & 19.20 & 8.46 \\
\hline $\begin{array}{l}1.53 \\
1.65\end{array}$ & $\begin{array}{l}19.43 \\
18.52\end{array}$ & $\begin{array}{l}8.46 \\
8.60\end{array}$ \\
\hline $\begin{array}{l}1.02 \\
.715\end{array}$ & 22.74 & 7.42 \\
\hline $\begin{array}{l}2.15 \\
0.63\end{array}$ & $\begin{array}{l}\begin{array}{l}16.65 \\
25.47\end{array}\end{array}$ & $\begin{array}{l}8.86 \\
6.06\end{array}$ \\
\hline & & \\
\hline $\begin{array}{l}1.24 \\
0.77\end{array}$ & 25.00 & $\begin{array}{l}8.06 \\
6.33\end{array}$ \\
\hline $\begin{array}{l}0.85 \\
0.95\end{array}$ & $\begin{array}{l}24.16 \\
24.06\end{array}$ & $\begin{array}{l}6.76 \\
7.05\end{array}$ \\
\hline 0.99 & 24.28 & 6.91 \\
\hline $\begin{array}{l}0.58 \\
0.61\end{array}$ & $\begin{array}{l}25.95 \\
26.05\end{array}$ & $\begin{array}{l}5.85 \\
5.39\end{array}$ \\
\hline 0.66 & 24.87 & 6.35 \\
\hline $\begin{array}{l}0.81 \\
0.92\end{array}$ & $\begin{array}{l}24.16 \\
2.32\end{array}$ & $\begin{array}{l}6.86 \\
7.29\end{array}$ \\
\hline $\begin{array}{l}1.15 \\
1.55\end{array}$ & 21.61 & 7.94 \\
\hline $\begin{array}{l}1.53 \\
1.50\end{array}$ & $\begin{array}{l}20.60 \\
2237\end{array}$ & \\
\hline 1.35 & 21.68 & 8.10 \\
\hline $\begin{array}{l}1.53 \\
1.45\end{array}$ & $\begin{array}{l}\begin{array}{l}21.55 \\
21.20\end{array} \\
20\end{array}$ & $\begin{array}{l}8.17 \\
8.24\end{array}$ \\
\hline 1.38 & 22.01 & \\
\hline $\begin{array}{l}1.53 \\
1.47\end{array}$ & 21.90 & 8.00 \\
\hline & 21.33 & 8.19 \\
\hline $\begin{array}{l}1.21 \\
1.21\end{array}$ & $\begin{array}{l}211.68 \\
21.38\end{array}$ & $\begin{array}{l}8.80 \\
8.04\end{array}$ \\
\hline 1.43 & 22.08 & 7.89 \\
\hline $\begin{array}{l}1.41 \\
1.11\end{array}$ & 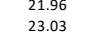 & $\begin{array}{l}7.911 \\
7.17\end{array}$ \\
\hline $\begin{array}{l}1.18 \\
1119\end{array}$ & $\begin{array}{l}22.81 \\
227.78\end{array}$ & 7.33 \\
\hline & & \\
\hline 1.48 & 20.71 & 8.34 \\
\hline $\begin{array}{l}1.111 \\
1.29\end{array}$ & $\begin{array}{l}21.81 \\
20.95\end{array}$ & $\begin{array}{l}7.83 \\
8.20\end{array}$ \\
\hline & & \\
\hline 1.83 & 19.11 & \\
\hline $\begin{array}{l}1.68 \\
1.34\end{array}$ & 19.70 & 8.57 \\
\hline $\begin{array}{l}1.34 \\
1.44\end{array}$ & $\begin{array}{l}20.62 \\
20.64\end{array}$ & $\begin{array}{l}8.30 \\
8.22\end{array}$ \\
\hline 1.84 & 18.92 & 8.66 \\
\hline $\begin{array}{l}1.19 \\
1.78\end{array}$ & $\begin{array}{l}199.76 \\
18.76\end{array}$ & $\begin{array}{l}8.56 \\
8.65\end{array}$ \\
\hline 1.28 & 20.67 & 8.04 \\
\hline $\begin{array}{l}1.45 \\
1.31\end{array}$ & $\begin{array}{l}20.64 \\
20.51\end{array}$ & $\begin{array}{l}8.24 \\
8.10\end{array}$ \\
\hline $\begin{array}{l}1.25 \\
1.27\end{array}$ & 20.81 & 7.98 \\
\hline 1.34 & & \\
\hline 1.34 & 20.40 & 8.02 \\
\hline & $\begin{array}{l}20.16 \\
20.47\end{array}$ & $\begin{array}{l}8.10 \\
809\end{array}$ \\
\hline 0 & 17.86 & \\
\hline & & \\
\hline
\end{tabular}

